\title{
THE WILd LIFE OF Tick-BORNe Pathogens
}

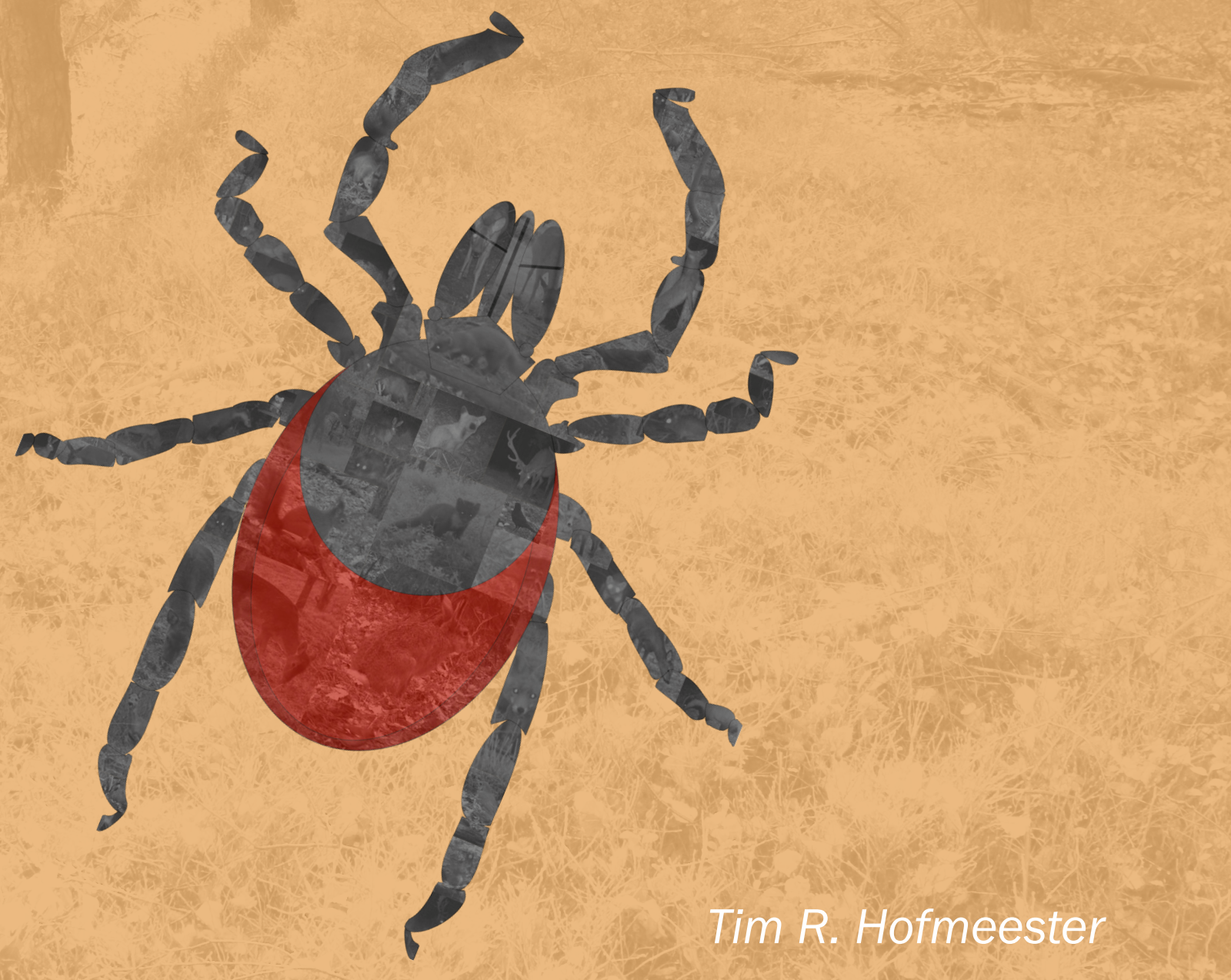




\section{The Wild Life of Tick-borne Pathogens}

Tim R. Hofmeester 


\section{Thesis COMMITTeE}

\section{Promotor}

Prof. Dr H.H.T. Prins

Professor of Resource Ecology

Wageningen University

\section{Co-promotor}

Dr S.E. van Wieren

Associate professor, Resource Ecology Group

Wageningen University

\section{Other members}

Prof. Dr M. Begon, University of Liverpool, United Kingdom

Prof. Dr M.C.M. de Jong, Wageningen University

Dr C.J.M. Koenraadt, Wageningen University

Prof. Dr X. Lambin, University of Aberdeen, Scotland

This research was conducted under the auspices of the C.T. de Wit Graduate School for Production Ecology and Resource Conservation. 


\title{
The Wild Life of Tick-borne Pathogens
}

\author{
Tim R. Hofmeester
}

\section{THESIS}

submitted in fulfilment of the requirements for the degree of doctor at Wageningen University by the authority of the Rector Magnificus

Prof. Dr A.P.J. Mol, in the presence of the

Thesis Committee appointed by the Academic Board to be defended in public on Monday 5 December 2016 at 4 p.m. in the Aula. 
Tim R. Hofmeester

The Wild Life of Tick-borne Pathogens,

224 pages.

PhD thesis, Wageningen University, Wageningen, NL (2016)

With references, with summaries in English and Dutch

ISBN: 978-94-6257-969-9

DOI: $10.18174 / 393112$ 



\section{CONTENTS}

1. General Introduction ...............................................................................

2. Few Vertebrate Species Dominate the Borrelia burgdorferi s.I. Life Cycle..19

3. A Simple Method for Estimating the

Effective Detection Distance of Camera Traps

4. Deer Presence rather than Abundance

Determines the Population Density of the Sheep Tick (Ixodes ricinus) ...63

5. Determinants of Tick (Ixodes ricinus) Burden on Rodent Populations in Dutch Forests.................................................79

6. Cascading Effects of Changes in Vertebrate Assemblages on Rodent-transmitted Tick-borne Disease Risk .........................................97

7. No Support for a Dilution Effect of Host Species Richness on Tick-borne Disease Risk in Europe...............119

8. Synthesis: From Biodiversity to

Behaviour of Specific Species in Disease Ecology ................................143

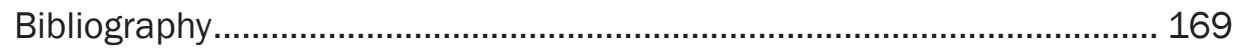

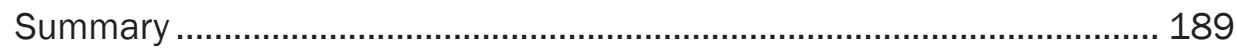

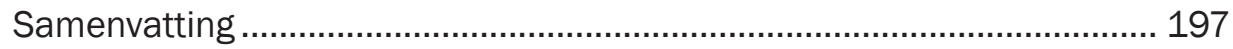

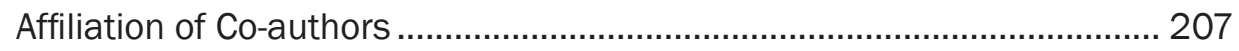

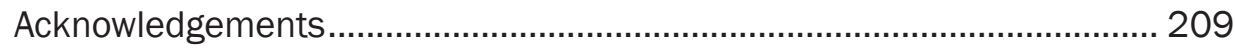

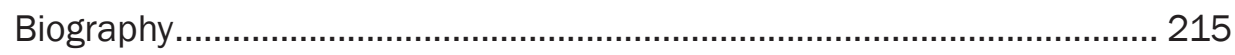

PE\&RC Training and Education Statement ............................................. 221 


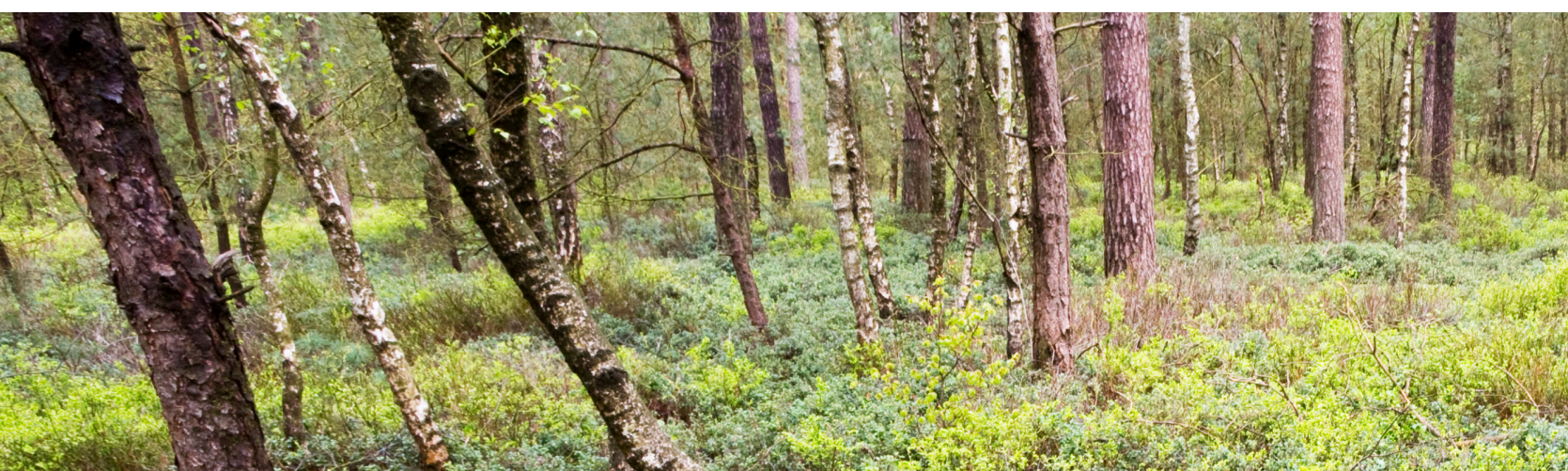

hello sir, hello miss

my name is Ixodes ricinus

now we meet I hope it's nice

cause your juicy body is a paradise

most of the time I hide out of sight

but some of you creatures I'm anxious to bite

then I can suck your blood to my delight

so as a species we are all right

if you want to know more

about me and the ecology of my diseases

please read on in this fine thesis

and learn what makes me tick and roar

Ronald Hofmeester 


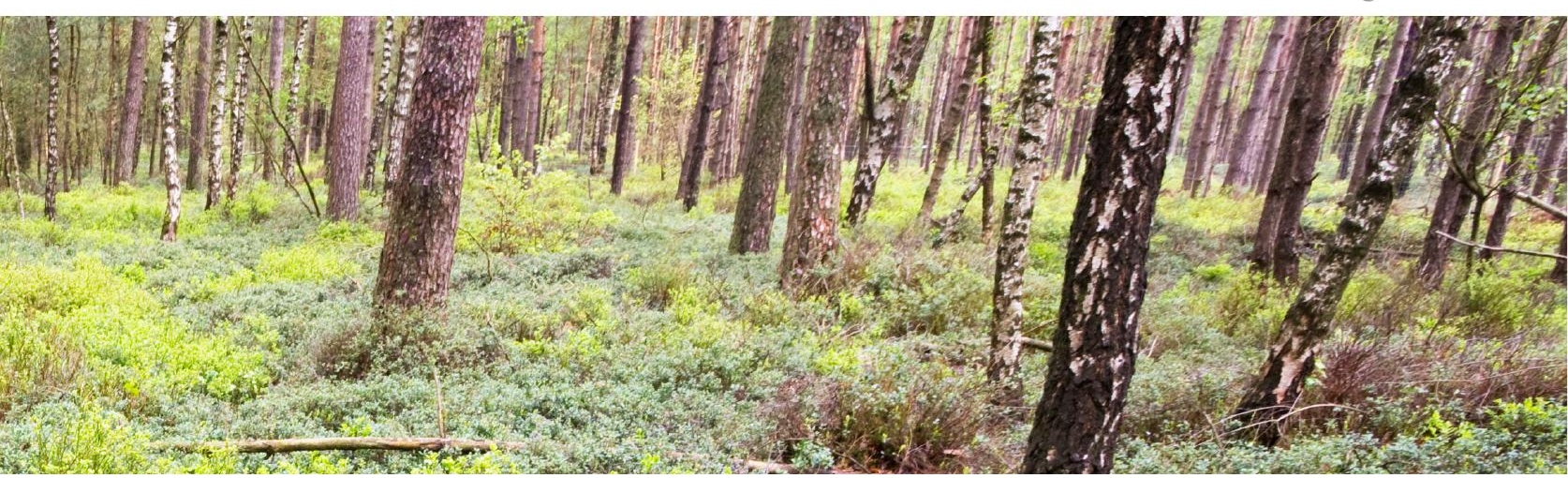

\section{GENERAL INTRODUCTION}

Zoonotic vector-borne diseases pose an increasing threat to human health as they comprised approximately $25 \%$ of the emerging infectious diseases in the last decades ${ }^{1,2}$. Most vector-borne diseases are zoonotic, which means that they are caused by pathogenic microorganisms that reside in wildlife or husbandry animal hosts, and are transmitted to humans by the bite of a vector, generally a bloodsucking arthropod ${ }^{3}$. One of the prerequisites for a zoonotic vector-borne disease to affect humans is that both vector and pathogen are generalists: The vector has to parasitize both animal hosts and humans, and the pathogen has to be able to reside in three types of host: humans, animal reservoirs and vectors ${ }^{4}$. Many ectoparasites are generalist feeders, which has resulted in ectoparasite species acting as vectors for diseases such as Lyme borreliosis, human epidemic typhus, bubonic plague and relapsing fever ${ }^{3,5,6}$. 


\section{0 | Chapter 1}

\section{Ectoparasite population dynamics}

To understand the role of ectoparasites in pathogen transmission, we need information about the population dynamics of these parasites and the factors determiningthesedynamics. Duetotheirparasiticnature, ectoparasite populations are dependent on the availability of hosts and therefore characteristics of these hosts will have an effect on parasite population dynamics ${ }^{7-9}$. For example, the spatial behaviour of hosts determines the spatial distribution of parasites ${ }^{10,11}$. An important characteristic determining the importance of host species or individuals for maintaining parasites is parasite burden. Parasite burden is determined by 1 ) the number of parasites in the environment, and 2) the spatial behaviour of a host, which together determine the encounter rate of parasites and hosts ${ }^{12}$, and 3) the resistance or tolerance of hosts towards the parasite ${ }^{13}$.

As the number of parasites on a host is dependent on the number of parasites in the environment and vice versa, there is a positive feedback between host and parasite density ${ }^{14}$. This positive feedback is especially striking for specialist ectoparasites that feed on one host species ${ }^{15}$. The density of generalist parasites that infest multiple host species depends on the densities of the different host species and their parasite burden ${ }^{16}$. Differences between host species in spatial behaviour - related to body size, home range size and habitat preference - or in resistance or tolerance - related to immune response and grooming behaviour - will affect their parasite burden and hence parasite population dynamics of generalist parasites ${ }^{17-20}$. Therefore, the species composition of the host assemblage is an important determinant of parasite population dynamics.

\section{Pathogen population dynamics}

For generalist ectoparasites that function as disease vectors, differences in parasite burden between host species do not only determine vector population dynamics, butalso the infection prevalence of pathogens in these vectors ${ }^{9,21}$. Many vector-borne pathogens are amplified by vertebrate hosts and transmitted from vector to host to vector (trans-stadial or horizontal transmission), while some are transmitted from vector to offspring (trans-ovarial transmission) ${ }^{22}$. For pathogens that are only transmitted by trans-stadial transmission, a host individual needs to get infected by an infected vector first before it can infect feeding vectors. The chance that a host individual gets infected therefore depends on the likelihood 
of feeding an infected vector and hence its vector burden. The ability of the host to infect feeding vectors after it gets infected with the pathogen depends on the tolerance or resistance of the host to both the vector and the pathogen ${ }^{23,24}$. Therefore, host species differ in their ability to transmit pathogens to vectors, often referred to as (realized) reservoir competence ${ }^{25}$.

When a generalist vector is maintained by an assemblage of vertebrate host species that differ in their vector burden and their realized reservoir competence, the distribution of vectors over the host species will determine vector density and vector-borne pathogen prevalence in the vectors. The distribution of vectors over hosts is dependent on the host preference of vectors ${ }^{26}$ and the relative abundance of the different host species ${ }^{27}$. The preference of vectors for certain host species is a very important determinant of the distribution of vectors over hosts for highly mobile vectors such as mosquitoes ${ }^{28}$. In contrast, the relative abundance of hosts might be more important in determining the distribution of vectors over hosts for vectors that are less mobile such as ticks ${ }^{21}$.

Both vectors and pathogens are likely to coevolve with host species that are widespread and abundant ${ }^{29}$, and therefore these host species are more likely to be the preferred hosts for both vector and pathogen. This might explain the high realized reservoir competence of widespread host species for many vector-borne pathogens ${ }^{26,30}$. The presence of other host species might 'dilute' the number of vectors feeding on these widespread host species with a high realized reservoir competence, which has led to the proposition of the 'dilution effect of host species richness' hypothesis ${ }^{3}$. The dilution effect hypothesis predicts that host assemblages with low species richness will have many species which have a high realized reservoir competence. At the same time, the hosts that are added to the assemblage when species richness increases are predicted to have a lower realized reservoir competence. There are several mechanisms by which the presence of other hosts can 'dilute' pathogen prevalence: 1) it can reduce the encounter probability between host species with a high realized reservoir competence and vectors (encounter reduction), 2) it can reduce the number of vectors in the environment (vector regulation), and 3 ) it can reduce the density of host species with a high realized reservoir competence (reservoir host regulation ${ }^{31}$. In contrast, the addition of hosts might increase vector density, and therefore the vector burden of reservoir-competent host species (vector augmentation $)^{32}$. There are multiple theoretical studies showing the possibility of mechanisms by which the addition of hosts with a low realized reservoir 


\section{2 | Chapter 1}

competence can decrease vector-borne pathogen prevalence ${ }^{27,33}$, but there are only a few studies that have investigated these mechanisms empirically ${ }^{34}$. Therefore, it is important to perform empirical studies to better understand the interactions between different host species and the effect of these interactions on vector and vector-borne pathogen population dynamics.

\section{Ticks and tick-borne pathogens}

The Sheep tick (Ixodes ricinus) is the main disease vector in Europe, carrying pathogens which cause human diseases such as Lyme borreliosis, tick-borne encephalitis, babesiosis and human granulocytic anaplasmosis ${ }^{35}$. The life cycle of $I$. ricinus consists of three active life stages, larva, nymph and adult, which all need a bloodmeal from a vertebrate host in order to moult to the next stage or to lay eggs, and all stages feed from a large array of host species ${ }^{36,37}$ (Figure 1.1). Ixodes ricinus is sensitive to desiccation and is mostly found in forested areas throughout Europe ${ }^{36}$. Within forests, it is dependent on the availability of hosts

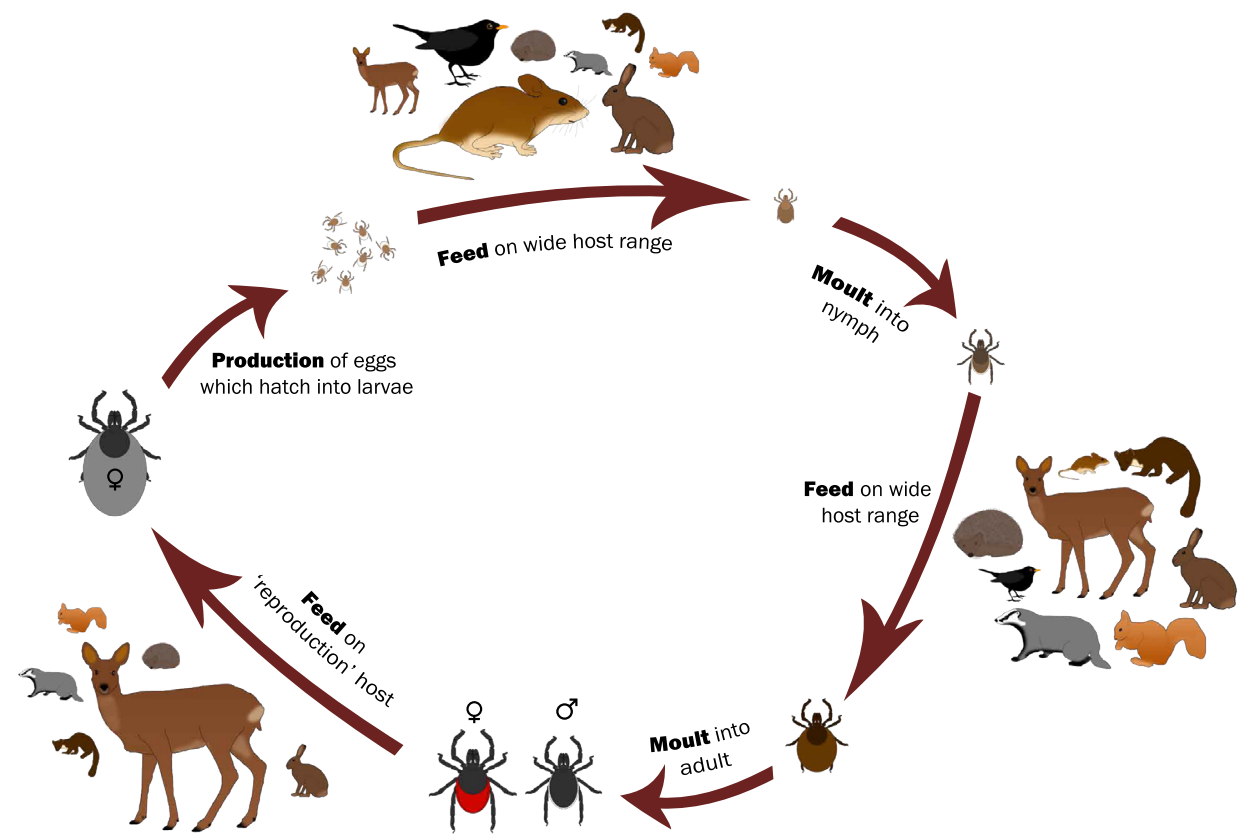

Figure 1.1. Schematic representation of the life cycle of Ixodes ricinus. Ixodes ricinus immature stages feed on a large range of host species, including shrews, voles, mice, squirrels, hedgehogs, lagomorphs, mustelids and ungulates, while adult female $I$. ricinus feed on a smaller range of hosts, including hedgehogs, hares, and ungulates. Pictures of single species represent groups of taxonomically related species. The size of the host picture represents the theorized importance of the host taxon for that life stage of $I$. ricinus. 
A)

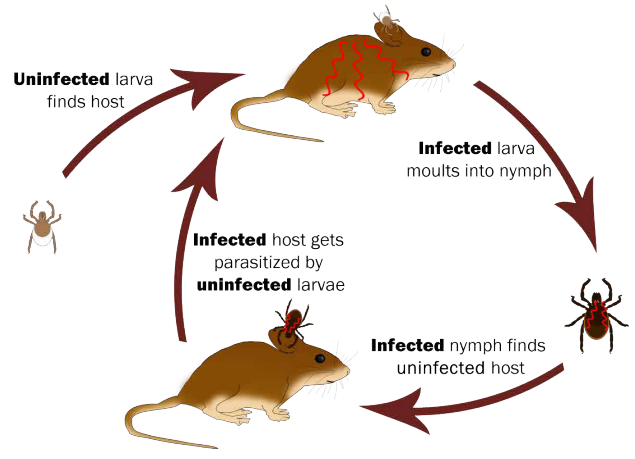

B)

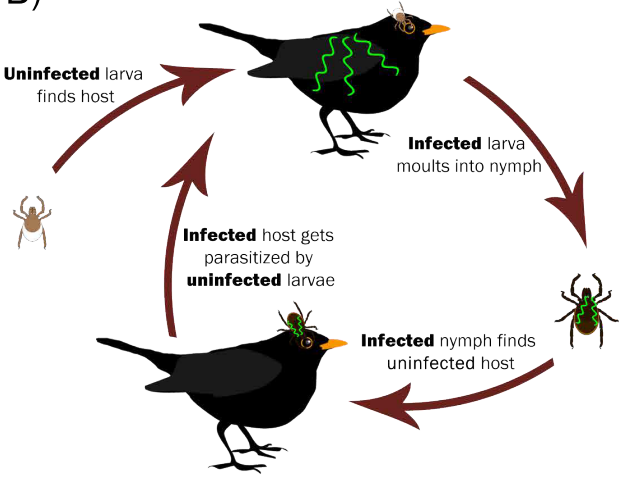

Figure 1.2. Schematic representation of the transmission cycle of A) rodent-transmitted Borrelia spp. (red) and B) bird-transmitted Borrelia spp. (green). Ixodes ricinus larvae get infected by feeding from an infected host, after which they moult into an infected nymph. This infected nymph can now infect uninfected hosts from which it feeds, completing the transmission cycle.

for all stages of the life cycle, where the presence and abundance of deer, as hosts for the adult stage, is theorized to be an important determinant of I. ricinus density ${ }^{38,39}$.

Ixodes ricinus encounters a host by sitting on the vegetation and waiting, called 'questing'8. The different life stages quest at different heights, which might influence which hosts the different life stages encounter ${ }^{40}$. Due to this passive strategy of encountering a host, the distribution of ticks over hosts will mainly be determined by the relative abundance of different host species ${ }^{27}$, making it an ideal system to study the importance of vertebrate assemblage composition on vector density and vector-borne pathogen prevalence.

In Western Europe, there are several bacterial pathogens that are transmitted by I. ricinus including Borrelia burgdorferi sensu lato (s.l.), the causative agent of Lyme borreliosis ${ }^{41}$, Anaplasma phagocytophilum, the causative agent of human granulocytic anaplasmosis ${ }^{42}$, Borrelia miyamotoi, the causative agent of acute febrile illness ${ }^{43}$, and Candidatus Neoehrlichia mikurensis, the causative agent of neoehrlichiosis ${ }^{44}$. Of these, Lyme borreliosis is the most abundant tick-borne disease, which has increased over the last few decades ${ }^{45}$. Borrelia burgdorferi s.l. is a species complex that consists of 18 different genospecies worldwide ${ }^{46,47}$. Each genospecies has evolved in a different way to circumvent the immune response of the hosts it resides in, which has resulted in host specificity of the different genospecies ${ }^{48}$. For example, $B$. afzelii has specialized on rodents, while 


\section{4 | Chapter 1}

B. garinii has specialized on birds ${ }^{49}$ (Figure 1.2). Similarly, A. phagocytophilum, B. miyamotoi, and Ca. N. mikurensis are transmitted by specific vertebrate host species $^{50,51}$. All of these pathogen species are amplified by hosts via trans-stadial transmission, and host species differ in their realized reservoir competence for the different pathogen species. Therefore, changes in the distribution of ticks over different hosts as a result of changes in vertebrate assemblage composition is expected to change the prevalence of these pathogens in questing ticks, making $I$. ricinus and its hosts and pathogens an ideal system to test the dilution effect hypothesis for multiple pathogens within the same system.

\section{Thesis outline}

The main objective of this thesis was to better understand the role of different vertebrate host species in maintaining $I$. ricinus populations and in infecting I. ricinus larvae with different tick-borne pathogens. For this I performed both a systematic review and an extensive field study. In Chapter 2, I present the systematic review in which I analysed data on interactions between I. ricinus, vertebrate host species and $B$. burgdorferi s.l. to collate the existing knowledge on the importance of different host species for maintaining I. ricinus and $B$. burgdorferi s.l. populations.

For the field study, I did a cross-sectional study and an exclosure experiment. For the cross-sectional study I selected twenty plots of 1 hectare (ha) in nineteen forested sites in the Netherlands (Figure 1.3). In one site, Enkhout, I selected two plots $150 \mathrm{~m}$ apart of which one was located within a 3 ha forest patch which was fenced three years prior to sampling to exclude all large herbivores. I sampled eleven plots in 2013 and nine in 2014. In these plots I sampled for questing ticks by blanket dragging and used live traps to estimate small mammal densities and tick burden on rodents. I decided to use camera traps as a standardized method to study all other vertebrate species in a quantitative way. Because, in a way, camera traps hanging on a tree and photographing animals can be seen as questing ticks encountering hosts. By sampling each plot only once, I assumed that relative abundances of vertebrate species in the sampling year were representative of the relative abundances of those species the year prior to sampling.

Camera traps are cameras that are triggered by a passive infrared (PIR) sensor that registers a difference in thermal infrared ${ }^{52}$ (Figure 1.4). In my case this meant 
that camera traps would start to take photographs as soon as a warm-blooded animal walked past and was detected by the PIR sensor ${ }^{53}$. The sensitivity of the PIR sensor is, among other things, dependent on the surface temperature of the animal walking past, which is different for animals of different sizes, as bigger species omit more heat than smaller species ${ }^{54}$. Therefore, PIR sensors detect bigger species at larger distances than smaller species. Due to this difference,

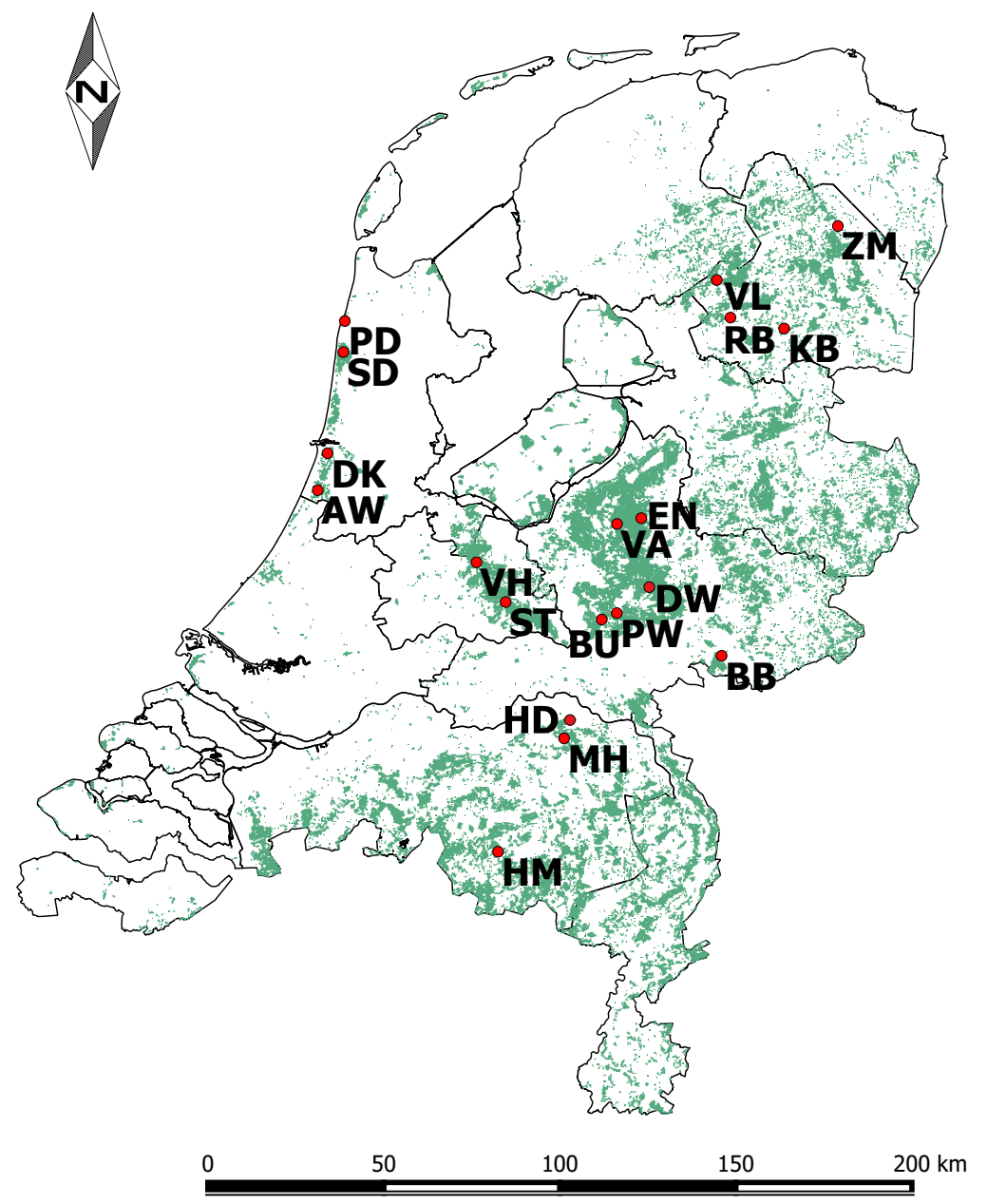

Figure 1.3. Map of the Netherlands with the nineteen sites where I conducted the cross-sectional field study. $\mathrm{AW}=$ Amsterdamse Waterleiding Duinen, $\mathrm{BB}=$ Bergherbos, $\mathrm{BU}=$ Buunderkamp, DK = Duin en Kruidberg, DW $=$ Deelerwoud, $\mathrm{EN}=$ Enkhout, $\mathrm{HD}=$ Herperduin, $\mathrm{HM}=$ Halfmijl, $\mathrm{KB}=$ Kremboong, $\mathrm{MH}=$ Maashorst, $\mathrm{PD}=\mathrm{Pet}-$ temerduinen, $\mathrm{PW}=$ Planken Wambuis, RB = Landgoed Rheebruggen, $\mathrm{SD}=$ Schoorlse duinen, $\mathrm{ST}=\mathrm{Stameren}$, $\mathrm{VA}=$ Valenberg, $\mathrm{VH}=$ Vijverhof, $\mathrm{VL}=$ Landgoed Vledderhof, $\mathrm{ZM}=$ Zwanemeerbos. 


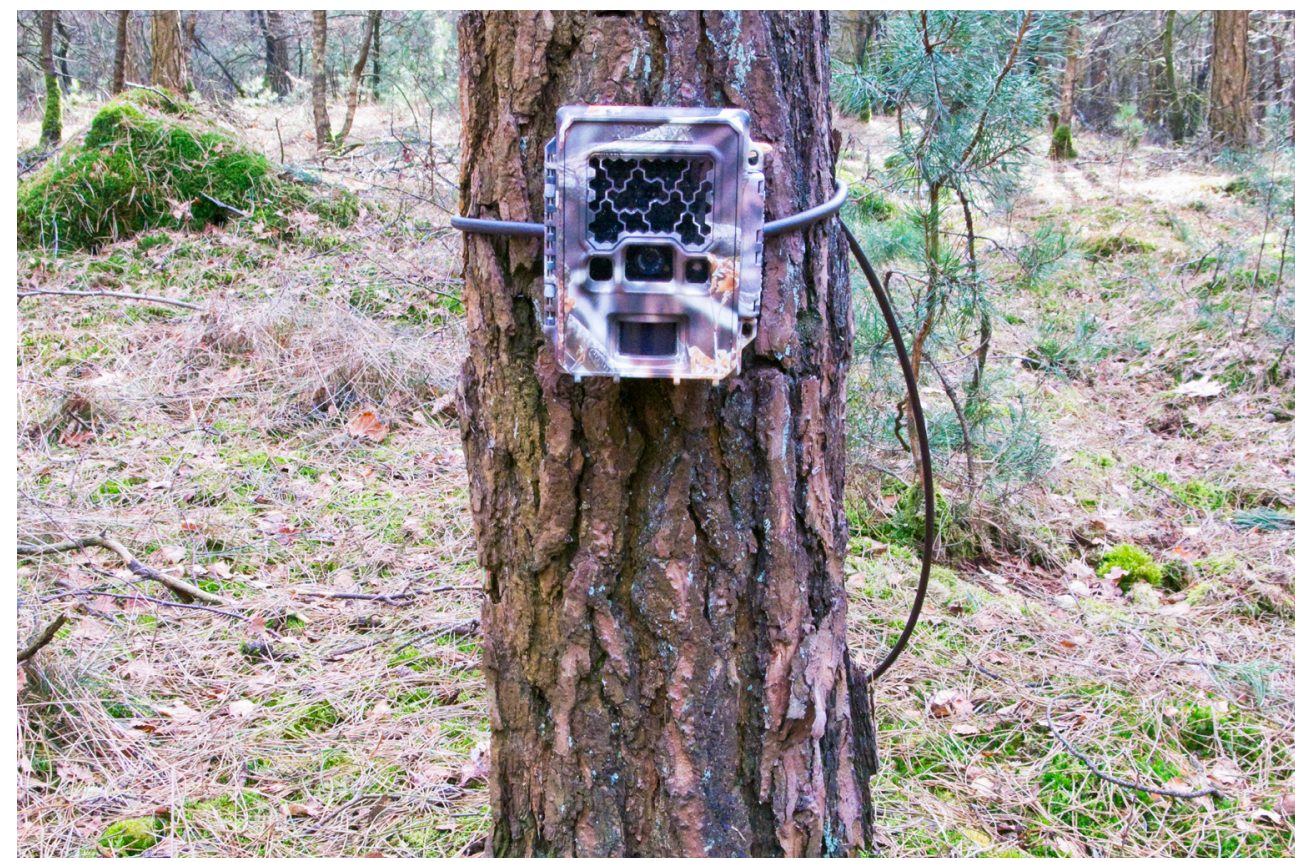

Figure 1.4. Camera trap (Reconyx HC500) attached to a tree. The camera lens is situated in the middle, with an infrared flash on top of it and the passive infrared sensor below the lens.

I needed to correct camera trap data to be able to compare the numbers of photographs taken from different animal species. This can be done by calculating the effective detection distance (EDD) of camera traps for each species ${ }^{54}$. However, established methods to estimate EDD were labour intensive ${ }^{54}$. To be able to perform the fieldwork - camera trapping and tick dragging - in multiple plots in one day, I needed an easier and quicker method to estimate EDD, which I present in Chapter 3.

I used this new method to correct photographic capture rates - as a measure of relative abundance - of all animal species that were photographed in the twenty 1-ha plots. It is widely assumed that $I$. ricinus density is determined by deer abundance, but empirical evidence is scarce ${ }^{38,55}$. Therefore, I performed a deer exclusion experiment using a Before - After Control - Impact design with four pairs of 0.75 -ha forest plots in a forest near Apeldoorn, the Netherlands. I used the data from this experiment and the data from the cross-sectional study to investigate the correlation between I. ricinus densities and the presence and relative abundance of deer (Chapter 4 ).

To better understand how different factors affect tick burden on hosts, I used 
the data from the cross-sectional study to investigate how intrinsic (e.g., species, body mass and sex of the host) and extrinsic (e.g., abundance of other hosts in the plot) factors were correlated with tick burden on rodents (Chapter 5). In Chapter 6, I used path analysis to study the indirect relationship of the relative abundance of deer and predators and rodent density with the density of infected nymphs for tick-borne pathogen species that are transmitted by rodents (B. afzelii, B. miyamotoi, and Ca. N. mikurensis), via tick burden on rodents. By doing this I could test for empirical evidence for encounter reduction, vector augmentation and reservoir host regulation.

Using all data collected in the cross-sectional study, I tested for correlations of the infection prevalence of seven tick-borne pathogen species (A. phagocytophilum, B. afzelii, B. burgdorferi sensu stricto (s.s.), B. garinii, B. valaisiana, B. miyamotoi, and $\mathrm{Ca}$. N. mikurensis) in questing nymphs and the density of infected nymphs with the abundance of reservoir-competent and reservoir-incompetent hosts (Chapter 7). I also tested if the abundances of reservoir-competent and reservoir-incompetent hosts were correlated with host species richness to test for empirical evidence for a dilution effect of host species richness for tick-borne pathogens on a small spatial scale in Europe. Furthermore, I used data from a recent systematic review ${ }^{56}$ to study the correlation of Lyme borreliosis incidence with host species richness on a large spatial scale in Europe. In Chapter 8 I synthesize and discuss the results of all previous chapters and relate the results of this thesis to a broader ecological context. 
tick tock

ticks the tick clock

what do we need

rodents, birds and deer

for our different stages, indeed

tick tock

passes the time on the Lyme clock

where does it breed

in ticks on plenty mice, or in scanty thrush

it copious succeeds

Ronald Hofmeester 


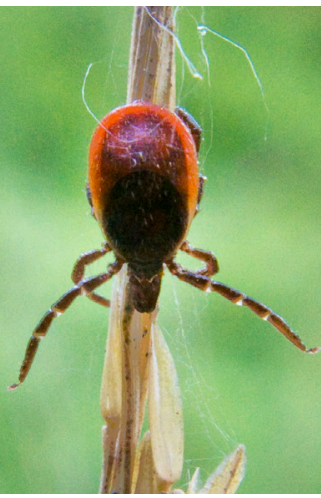

T.R. HOFMEESTER, E.C. COIPAN, S.E. VAN WIEREN, H.H.T. PRINS, W. TAKKEN \& H. SPRONG Environmental Research Letters 11: 043001

\section{Few Vertebrate Species Dominate the Borrelia BURGDORFERI S.L. LIFE CYCLE}

In the northern hemisphere, ticks of the Ixodidae family are vectors of diseases such as Lyme borreliosis, Rocky Mountain spotted fever and tick-borne encephalitis. Most of these ticks are generalists and have a three-host life cycle for which they are dependent on three different hosts for their blood meal. Finding out which host species contribute most to maintaining ticks and the pathogens they transmit, is imperative in understanding the drivers behind the dynamics of a disease. We performed a systematic review to identify the most important vertebrate host species for Ixodes ricinus and Borrelia burgdorferi s.l. as a well-studied model system for tick-borne diseases. We analysed data from 66 publications and quantified the relative contribution of 15 host species. We found a positive correlation between host body mass and tick burdens for the different stages of I. ricinus. We show that nymphal burdens of host species are positively correlated with infection prevalence with $B$. burgdorferi s.I., which is again positively correlated with the realized reservoir competence of a host species for $B$. burgdorferi s.l. Our quantification method suggests that only a few host species, which are amongst the most widespread species in the environment (rodents, thrushes and deer), feed the majority of $I$. ricinus individuals and that rodents infect the majority of I. ricinus larvae with B. burgdorferi s.I. We argue that small mammal-transmitted Borrelia spp. are maintained due to the high density of their reservoir hosts, while bird-transmitted Borrelia spp. are maintained due to the high infection prevalence of their reservoir hosts. Our findings suggest that Ixodes ricinus and Borrelia burgdorferi s.I. populations are maintained by a few widespread host species. The increase in distribution and abundance of these species, could be the cause for the increase in Lyme borreliosis incidence in Europe in recent decades. 


\section{0 | Chapter 2}

\section{Introduction}

Zoonotic vector-borne diseases pose an increasing threat to human health, as one-third of the emerging infectious diseases in the last decades was vectorborne $^{2}$. In the northern hemisphere, ticks of the Ixodidae family are vectors for human diseases such as Lyme borreliosis, Rocky Mountain spotted fever and tickborne encephalitis ${ }^{57}$. From these, the spirochaete complex Borrelia burgdorferi sensu lato ${ }^{58}$ (from here on referred to as $B$. burgdorferi), the causative agent of Lyme borreliosis and vectored by ticks of the Ixodes ricinus complex ${ }^{59}$, causes the majority of human disease cases ${ }^{60}$. Both in Europe and in North America, I. ricinus and $I$. scapularis populations have spread and increased in density in recent decades, most probably due to a multitude of man-made changes to the environment, which has resulted in an increase in Lyme disease incidence ${ }^{49,61}$.

Lyme disease risk is determined by multiple biological, environmental and societal factors $8,62,63$. These can be split into two distinct groups, 1) factors determining the number of questing lxodes ticks infected with $B$. burgdorferi, and 2) factors determining the level of human exposure to ticks ${ }^{39}$. In this review, we will focus on the first, with in particular the factors that determine the number of questing Ixodes ticks, and their infection with $B$. burgdorferi.

Both $I$. ricinus and $B$. burgdorferi are considered generalist parasites, as they utilize a multitude of host species ${ }^{37,46}$. These host species differ considerably in the numbers of ticks they feed, which differs between the different life stages of the tick ${ }^{36,64}$. Ixodes ricinus has three active life stages, larva, nymph and adult, that need a blood meal from a vertebrate host during each stage to moult to the next stage or to lay eggs ${ }^{36}$. Host species differ in their ability to infect $I$. ricinus larvae with different genospecies of B. burgdorferi. For example, B. afzelii is mainly transmitted by small mammals, while $B$. garinii is mainly transmitted by birds ${ }^{65,66}$, and even within genospecies, different host species differ in their ability to transmit $B$. burgdorferi ${ }^{24}$. Both the number of ticks a host can feed and the transmission of $B$. burgdorferi could be linked to general host characteristics, such as host body mass, which is related to both immunological and behavioural responses ${ }^{67-69}$, and could therefore influence both tick burden and reservoir competence for $B$. burgdorferi $i^{18,70,71}$.

The success of transmission and maintenance of $B$. burgdorferi in enzootic cycles depends, therefore, on the density and abundance of the various vertebrate host species. As the transmission of $B$. burgdorferi from one host to another is 
mediated by ticks, the distribution of the various genospecies is also dependent on the behaviour and feeding preference of the vector ticks. Thus, the resulting B. burgdorferi distribution in the questing ticks is a function of the densities of different host species, their capacity to feed ticks and their capacity to transmit the bacteria to those ticks. Therefore, it is important to summarize data on the distribution of ticks of different stages over different vertebrate host species and use these data to find patterns that could be related to the increase in disease risk due to indirect effects by human induced changes to the environment.

Although there have been several studies carried out on particular sites $^{72}$ or on various classes of vertebrates ${ }^{73}$, and some descriptive reviews have been published $^{62,74,75}$, there is no quantitative review that integrates data on a host assemblage comprising a wide range of vertebrate species. Here, we used a data driven approach to quantify the contribution of various vertebrate host species to feeding $I$. ricinus ticks, and transmitting $B$. burgdorferi to feeding larvae, to infer a mechanism that could support the apparent increase in Lyme borreliosis incidence in Europe. Furthermore, pinpointing the host species that are most important in feeding $I$. ricinus might aid in selecting host species to target intervention strategies ${ }^{76}$.

We compiled data on interactions between vertebrate hosts, I. ricinus and B. burgdorferi using a systematic review approach. For the species for which data were available that fulfilled our selection criteria, we looked for correlations between: 1) body mass and I. ricinus burden for the different stages, 2) nymphal burden and infection prevalence with $B$. burgdorferi, and 3) infection prevalence and realized reservoir competence for $B$. burgdorferi. We hypothesize that host species body mass is positively correlated to I. ricinus burden, as host species of greater body mass have a greater day range ${ }^{67}$ and are therefore more likely to encounter ticks in the vegetation. Furthermore, we hypothesize that the I. ricinus burden of a host species is positively correlated with the infection prevalence with $B$. burgdorferi as hosts that feed a large number of ticks are more likely to feed an infected tick and become infected. Lastly, we hypothesize that the average infection prevalence of a host species is positively correlated with the realized reservoir competence of a host species, as hosts that are more often infected are more likely to transmit the disease to a large number of larvae.

Next to these general patterns, we aimed to quantify the relative contribution of different host species in the maintenance of $I$. ricinus and $B$. burgdorferi. For this, we modified the framework proposed by Mather et al. ${ }^{77}$ to quantify 


\section{2 | Chapter 2}

the importance of different vertebrate species based on differences in density, I. ricinus burden and realized reservoir competence for $B$. burgdorferi. The original framework ${ }^{77}$ was created to quantify the relative importance of different host species in infecting I. scapularis larvae with B. burgdorferi in three study sites in North America. We modified the equations to quantify the relative importance of host species in the feeding of $I$. ricinus as well as the relative importance in infecting larvae with $B$. burgdorferi. As these equations need a vertebrate assemblage for their calculation, we used data from the literature search to create an assemblage including the most widespread vertebrate species occurring in most European forests.

\section{Methods}

We performed a literature search using PubMed, Web of Science and Scopus to review the parasitism of Ixodes ricinus on vertebrate host species, and the occurrence of Borrelia burgdorferi in vertebrate hosts and in the I. ricinus parasitizing them. We only considered European host species. Our most extensive search was done in PubMed, were we searched for publications in English and German. The last literature search was carried out in January 2015 and concerned the years 1945-2014. The search string used, and part of the selection procedure are given in Appendix 2.1. An additional screening of relevance concentrated on the type of data the publications included: field-derived or laboratory data. As we used the data for a quantification framework resembling a situation in the field, we chose only publications that contained field-derived data. Finally, we selected for papers including data on: 1) measurements of the tick burden on the vertebrate hosts, 2) measurements of host infection prevalence with $B$. burgdorferi, or 3) measurements of infection prevalence with $B$. burgdorferi in feeding ticks. Publications with incomplete or previously published data were excluded. All publications were reviewed by two different contributors (TRH and ECC) and the data extracted from each paper were checked twice.

\section{Data acquisition}

For each of the selected publications the following variables were extracted: location, number of hosts examined, number of hosts infested, number of hosts infected, numbers of $I$. ricinus of each stage per host, method of $B$. burgdorferi detection, type of tissue tested, number of samples (ticks or tissue) tested, 
number of samples (ticks or tissue) positive and genospecies of B. burgdorferi detected. These variables were the primary variables in our database, and were used for subsequent calculations. Difficulties in extracting data resulted from reported data that accounted for total number of ticks only (no stage mentioned) or combined observations of multiple host species. These papers were stored in the database, but not used for further analysis. To fill the database with values for the desired analyses, the following steps were carried out. If the number of infested or infected hosts or ticks in the study was not given, it was calculated, if possible, based on the number of samples examined and the reported infestation or infection prevalence. Similarly, if the number of ticks infesting the host animals was not given, then we would calculate it from the number of animals examined and their mean infestation. Most of the time, however, a total number of ticks from a specific stage collected from a total number of hosts was given, so we could calculate the average tick burden per stage. Only about one third of the publications (56/162) contained standard deviations, standard errors or confidence intervals for the parameters we were interested in, so we decided to calculate merely an average value and no other descriptive statistical parameters.

Publications were divided into separate records if the investigators examined 1) different host species, 2) hosts collected in different locations (if specified), 3) different tick stages, 4) different Borrelia genospecies, or 5) hosts tested also by xenodiagnosis. From publications in which the same ticks were examined with different methods for $B$. burgdorferi detection, leading to different results, only data obtained by PCR or sequencing were included. Records containing sequencing data were considered to have tested for the presence of all the B. burgdorferi genospecies described in the paper. Combining these records resulted in a database on tick burdens, host infection prevalence, and infection prevalence in feeding ticks per host species with data from 162 publications*.

\section{Summarizing the data}

As most studies presented only total numbers of animals and ticks studied, we calculated the average tick burden for each stage of tick for each host species as,

$$
B_{l_{i}}=\frac{\sum_{s=1}^{n} L_{i_{s}}}{\sum_{s=1}^{n} H_{i_{s}}}
$$

Equation 2.1 


\section{4 | Chapter 2}

where $B_{l i}$ is the mean per-capita larval burden of species $i, L_{i s}$ is the total number of larvae counted on host species $i$ in study $s$, and $H_{i s}$ is the total number of individuals of host species $i$ studied in study $s . L_{i s}$ can be substituted by the total number of nymphs counted on species $i$ in study $s\left(N_{i s}\right)$ or by the total number of adults counted on species $i$ in study $s\left(A_{i s}\right)$ in order to calculate the mean per-capita nymphal burden $\left(B_{n i}\right)$ or the mean per-capita adult burden $\left(B_{a i}\right)$, respectively. Our term tick burden is equal to the mean density of ticks as defined by Kahl et al. ${ }^{78}$.

Infection with B. burgdorferi was calculated as the sum of infections with the various genospecies and with untyped $B$. burgdorferi, counting the mixed infections only once. We only considered $B$. burgdorferi and not the individual genospecies to be able to use data produced before widespread use of molecular techniques, and to facilitate comparison between different host taxa.

We calculated two different measures of infection. For each host species we calculated the infection prevalence $\left.\left(I P_{b b i}\right)\right)$ of that species with $B$. burgdorferi as,

$$
I P_{b b_{i}}=\frac{\sum_{s=1}^{n} B B I H_{i_{S}}}{\sum_{S=1}^{n} H_{i_{S}}}
$$

Equation 2.2

where $I P_{b b i}$ is the infection prevalence of species $i$ with any genospecies of $B$. burgdorferi, $\mathrm{BBIH}_{\text {is }}$ is the total number of $\mathrm{B}$. burgdorferi infected host individuals of species $i$ in study $s$ and $H_{i s}$ is the total number of host individuals of species $i$ sampled in study s. Infection can be determined by either testing tissue samples from a host, or by testing I. ricinus for presence of B. burgdorferi after they were collected from a host in the field. As these can, potentially, result in very different values, both were calculated separately and were named 'tissue-derived' infection prevalence and 'tick-derived' infection prevalence, respectively. Our term infection prevalence is equal to the ratio of pathogen-exposed hosts versus non-exposed hosts ${ }^{78}$ and indicates the fraction of animals within a species that has been infected by $B$. burgdorferi.

Next to that, we calculated the realized reservoir competence $R C_{b b i}$, i.e., the proportion of blood fed larvae that become infected with B. burgdorferi ${ }^{21}$, which is comparable to the specific host infectivity as defined by Kahl et al. ${ }^{78}$, as,

$$
R C_{b b_{i}}=\frac{\sum_{s=1}^{n} B B I L_{i_{S}}}{\sum_{s=1}^{n} L_{i_{s}}}
$$

Equation 2.3 
where $R C_{b b i}$ is the realized reservoir competence of species $i$ for any genospecies of $B$. burgdorferi, $B B I L_{i s}$ is the total number of $B$. burgdorferi infected larvae sampled from host species $i$ in study $s$, and $L_{i s}$ is the total number of larvae tested from species $i$ in study s.

\section{Selection criteria}

In order to improve the data quality in our analysis, we selected data from our database using the following criteria: 1) a minimum of 20 individuals from a species at a location was studied, 2) a minimum of 50 larvae was tested to determine the realized reservoir competence, 3 ) the study was conducted within the activity period of $I$. ricinus, for which we excluded studies performed in December-February, and studies that were performed all year round without specifying numbers for the separate seasons and 4) the study was conducted within habitats in which I. ricinus normally resides, namely forest, forest ecotone and woodland. Lastly, we excluded studies that only considered migratory birds. This resulted in a dataset with data for 44 species from 66 publications.

Quantifying the role of species as hosts for I. ricinus and B. burgdorferi We used this dataset to quantify the role of fifteen species as hosts for $I$. ricinus and $B$. burgdorferi, using modifications of the framework proposed by Mather et al. ${ }^{77}$. The original formula was used to quantify the relative importance of host species in infecting larval I. scapularis with B. burgdorferi. We modified the original equation to be used to calculate the relative importance of host species in feeding the different stages of $I$. ricinus (Equation 2.4), as well as the relative importance of host species in infecting I. ricinus larvae with B. burgdorferi (Equation 2.5). For Equation 2.5, we separated the number of infected vectors produced by the host species ( $N_{s}$ in the original model) into two different parameters, $B_{l i}$ and $R C_{b b i}$. We did this to clarify the similarity between the two equations we used.

$$
\begin{aligned}
& R I_{l_{i}}=\frac{B_{l_{i}} \cdot D_{i}}{\sum_{j=1}^{n} B_{l_{j}} \cdot D_{j}} \\
& R I_{b b_{i}}=\frac{B_{l_{i}} \cdot D_{i} \cdot R C_{b b_{i}}}{\sum_{j=1}^{n} B_{l_{j}} \cdot D_{j} \cdot R C_{b b_{j}}}
\end{aligned}
$$




\section{6 | Chapter 2}

$R l_{l i}$ is the relative importance of host species $i$ in feeding the larval stage of $I$. ricinus, $B_{l i}$ is the mean per-capita larval burden of species $i$, and $D_{i}$ is the density $\left(\mathrm{km}^{-2}\right)$ in which species $i$ occurs. The $\sum B_{l j} \cdot D_{j}$ gives the total number of larvae fed by all species in the assemblage, as determined by their mean larval burden and their densities. $B_{l i}$ can be substituted by $B_{n i}$ or $B_{a i}$ to calculate the relative importance of a host species in feeding the nymphal $\left(R I_{n i}\right)$ and adult $\left(R I_{a i}\right)$ stages, respectively.

$R I_{b b i}$ is the relative importance of species $i$ in infecting larvae with any genospecies of $B$. burgdorferi and is equivalent to the relative reservoir capacity used by Kahl et al. ${ }^{78}$ or the reservoir potential of Mather et al. ${ }^{77}$. This parameter could also be seen as the relative contribution of a host species to the pool of infected nymphs. We did not calculate the relative importance of host species for the separate $B$. burgdorferi genospecies, because there were not enough data available for multiple host species in our selection.

As the quantification of the relative importance of a species is dependent on all species in an assemblage, we needed to select a number of host species to perform our calculations. In principle the equations can be used for any

Table 2.1. Host species considered in our model assemblage, their taxonomic group and the density that was used in the calculations.

\begin{tabular}{lll}
\hline Scientific name & Host taxonomic group & Density $\left(\mathrm{km}^{-2}\right)$ \\
\hline Bank vole (Myodes glareolus) & Rodent & 1200 \\
Blue tit (Cyanistes caeruleus) & Small bird & 200 \\
Chaffinch (Fringilla coelebs) & Small bird & 100 \\
Common chiffchaff (Phylloscopus collybita) & Small bird & 100 \\
Dunnock (Prunella modularis) & Small bird & 200 \\
Eurasian blackbird (Turdus merula) & Thrush & 200 \\
Eurasian blackcap (Sylvia atricapilla) & Small bird & 40 \\
European hedgehog (Erinaceus europaeus) & Medium-sized mammal & 1 \\
European robin (Erithacus rubecula) & Small bird & 80 \\
Field vole (Microtus agrestis) & Rodent & 1000 \\
Great tit (Parus major) & Small bird & 100 \\
Red fox (Vulpes vulpes) & Medium-sized mammal & 1 \\
Roe deer (Capreolus capreolus) & Ungulate & 11 \\
Song thrush (Turdus philomelos) & Thrush & 80 \\
Wood mouse (Apodemus sylvaticus) & Rodent & 1200 \\
\hline
\end{tabular}


specific area where local densities and tick burdens are known. To present the idea behind the framework, and to show some overall trends that we think might be true for any area, we selected a hypothetical assemblage of species. We chose a relatively diverse European forest vertebrate assemblage consisting of six mammalian and nine avian species (Table 2.1). All of these species occur regularly in north-western European forests or forest ecotones. Species were selected based on their area of distribution throughout Europe as described in published handbooks ${ }^{79,80}$ and on the number of individuals (minimum of 100 individuals) that was studied in the publications used for data acquisition. Densities of the species were collected from the same published handbooks used for determining the area of distribution. Although the relative importance is calculated per host species, we divided the host species into different species groups based on size and taxonomy (Table 2.1). We present only these broad group contributions, to show general patterns regardless of the contribution of individual species.

To test for the sensitivity of the framework to errors in the mean per-capita tick burden, we calculated the relative importance of the host groups for additional scenarios, in which the species composition of the host assemblage remained unaltered but in which the contribution of rodents, birds or ungulates varied by either doubling or halving the mean per-capita tick burden of these groups compared to the initial values, while all other parameters were kept constant.

\section{Statistical analysis}

To test for correlations between body mass, I. ricinus burden, $I P_{b b i}$ and $R C_{b b i}$ of the different host species, we performed a stepwise analysis. First, we tested for correlations between body mass and $I$. ricinus burden, second, we tested for correlations between $I$. ricinus burden and $I P_{b b i}$ and third, we tested for correlations between $I P_{b b i}$ and $R C_{b b i}$. For the species that were considered in our vertebrate assemblage for the quantification of the importance of different host species, we also tested for correlations between density and body mass. Statistical analyses were performed using R 3.2.281. All analyses were performed for each host taxa (birds, mammals and reptiles) separately.

We used log-log regressions to test for correlations between host body mass and $I$. ricinus burden for the three life stages using average body mass of the host species obtained from published handbooks ${ }^{79,80}$. This was done because the average tick burdens per life stage did not yield integers, which refrained us 


\section{8 | Chapter 2}

from performing generalized linear models using poisson or negative binomial distributions. Because of the presence of zeros, we added the lowest non-zero burden to the tick burdens ( 0.04 for larvae, and 0.01 for nymphs and adults) in order to calculate the $\log _{10}$. Due to the large variation in body size, we also $\log _{10}$ transformed host body mass. To give more weight to species that were studied more intensively, we weighted the log-log regression by sample size. We also used log-log regressions for testing the correlations between density and body mass of host species.

For both the infection prevalence and the realized reservoir competence we used a generalized linear model with a binomial distribution and a logit link function. For the infection prevalence we used the number of host individuals found infected and the number of host individuals found uninfected, using the tissue-derived data, to test for a correlation between infection prevalence and $\log _{10}$ transformed nymphal burden. We supplemented this dataset with tick-derived data for species for which tissue-derived data were missing. For the realized reservoir competence we used, per host species, the total number of larvae found infected and the total number of larvae found uninfected as reported in the selected papers, to test for a correlation between realized reservoir competence and logit infection prevalence. By using the binomial infected-non-infected data, we weighted the correlations by sample size. We tested if the models for realized reservoir competence could be improved by adding $\log _{10}$ body mass to the model, and compared the models using AICc values ${ }^{82}$ using the MuMIn package ${ }^{83}$.

To further analyse the impact of species averages taken from few studies with low sample sizes, we did a post-hoc analysis of leverage to check for the importance of single species in determining the regression coefficients. We calculated Cook's distance for all parameters in all analyses ${ }^{84}$. If a Cook's distance was larger than 0.5 , we checked the number of studies and the number of individuals on which the estimate was based.

\section{Results}

Tick burden, infection prevalence and realized reservoir competence of hosts

The 44 host species in our dataset differed ten to thousand-fold in I. ricinus burden, infection prevalence with $B$. burgdorferi and realized reservoir competence for B. burgdorferi (Table 2.2). Because we only had data on three 
reptile species, we performed analyses on mammals and birds only. Larval I. ricinus burden increased with host body mass for birds $\left(F_{1,18}=12.1, p=0.02, \beta=\right.$ 0.97 ) but not for mammals $\left(F_{1,15}=0.9, p=0.37\right)$. Nymphal I. ricinus burden was positively correlated to host body mass both for birds $\left(F_{1,18}=30.5, p<0.001, \beta\right.$ $=1.81$; Figure 2.1A $)$ and for mammals $\left(F_{1,15}=26.1, p<0.001, \beta=0.79\right.$; Figure 2.1D). Adult $I$. ricinus burden also increased with host body mass both for birds $\left(F_{1,18}=74.4, p<0.001, \beta=0.53\right)$ and for mammals $\left(F_{1,15}=73.9, p<0.001, \beta\right.$ $=1.15$ ).

The 25 host species for which we had data on infection prevalence with B. burgdorferi differed tenfold in infection prevalence (Table 2.2). Infection prevalence increased with nymphal $I$. ricinus burden both for birds (deviance

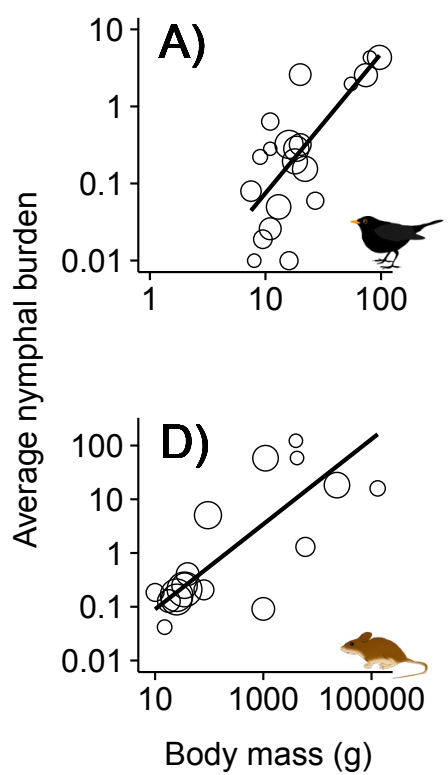

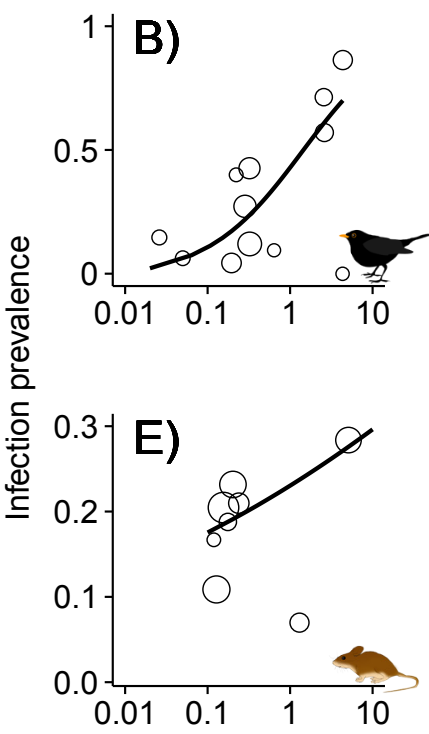

Average nymphal burden

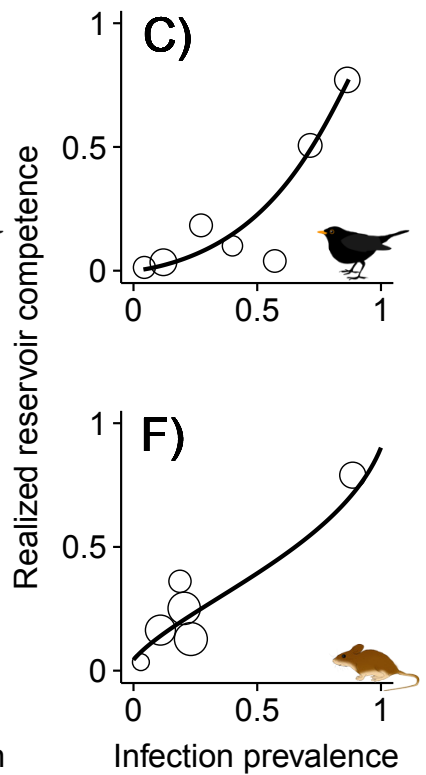

Figure 2.1. Correlations between nymphal I. ricinus burden, infection prevalence with $B$. burgdorferi and realized reservoir competence for $B$. burgdorferi. A) Log-log regression between host species body mass and average nymphal $I$. ricinus burden $(+0.01)$ for birds. B) Binomial regression between average nymphal I. ricinus burden and infection prevalence with $B$. burgdorferi for birds. C) Binomial regression between infection prevalence with $B$. burgdorferi and realized reservoir competence for $B$. burgdorferi for birds. D) Log-log regression between host species body mass and average nymphal I. ricinus burden for mammals. E) Binomial regression between average nymphal $I$. ricinus burden and infection prevalence with $B$. burgdorferi for mammals. F) Binomial regression between infection prevalence with $B$. burgdorferi and realized reservoir competence for $B$. burgdorferi for mammals. For each regression the sample size used to determine the value on the $y$-axis is represented by the size of the circles in the plot. 


\section{0 | Chapter 2}

difference $=199.1, p<0.001, \beta=1.76$; Figure 2.1B) and for mammals (deviance difference $=24.6, p<0.001, \beta=0.34$; Figure 2.1E). Of the 17 host species for which we had data on the realized reservoir competence for $B$. burgdorferi, 14 also had data on infection prevalence (Table 2.2). In these species, realized reservoir competence for $B$. burgdorferi increased with infection prevalence both for birds (deviance difference $=1048.2, p<0.001, \beta=1.29$ Figure $2.1 \mathrm{C}$ ) and for mammals (deviance difference $=903.7, p<0.001, \beta=0.72$; Figure 2.1F). For both groups, the model improved significantly by adding $\log _{10}$ body mass, which was positively correlated to realized reservoir competence in birds $(\triangle \mathrm{AICc}=59.7$, $\left.p<0.001, \beta_{\mathbb{P}}=0.54, \beta_{\text {body mass }}=2.88\right)$, and negatively correlated to realized reservoir competence in mammals $\left(\triangle \mathrm{AICC}=149.6, \mathrm{p}<0.001, \beta_{\mathrm{IP}}=1.15, \beta_{\text {body }}\right.$ mass $=-3.13$ ).

Post-hoc analyses of leverage indicated that for most analyses there were one or two species with a Cook's distance $>0.5$. However, most of the time these were the estimates which we gave a higher weight based on high sample size. In the few instances that species with a low sample size (less than 100 individuals) and low number of studies (less than three studies) had a high Cook's distance, omitting these species in the analysis only increased the fit. The only exception was the analysis of realized reservoir competence for mammals, for which omitting the data for Eliomys quercinus and Sorex araneus decreased the fit of the model including only infection prevalence with $B$. burgdorferi (deviance difference $=$ $0.25, p=0.62$ ). However, excluding these two species from the model including both infection prevalence with $B$. burgdorferi and $\log _{10}$ body mass resulted in a similar result as for all species, albeit with slightly different parameter estimates (deviance difference $=96.91, \mathrm{p}<0.001, \beta_{\mathbb{I P}}=0.76, \beta_{\text {bodymass }}=-3.80$ ). 
Few Vertebrate Species Dominate the B. burgdorferi Life Cycle | 31

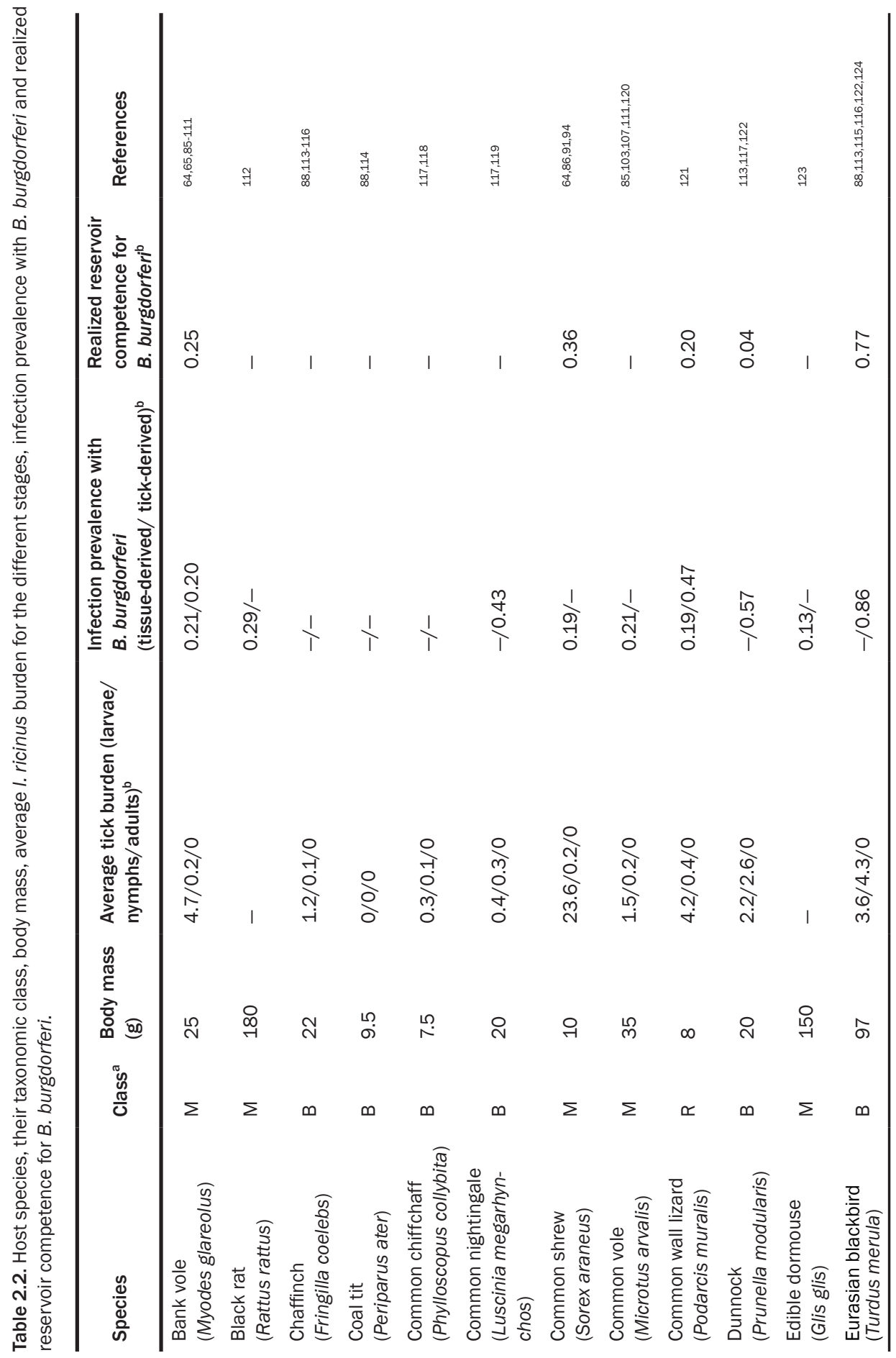




\section{2 | Chapter 2}

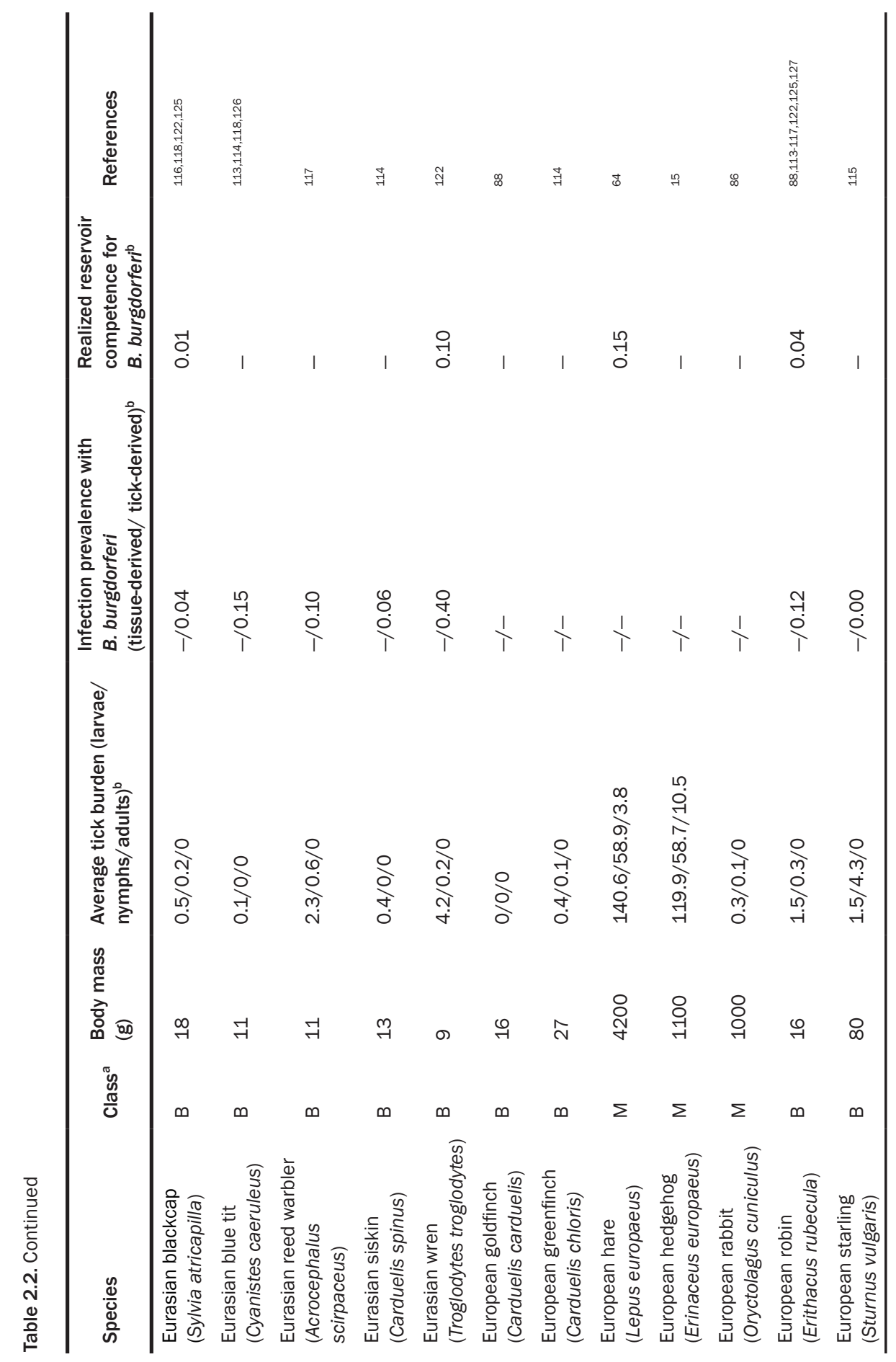


Few Vertebrate Species Dominate the B. burgdorferi Life Cycle | 33

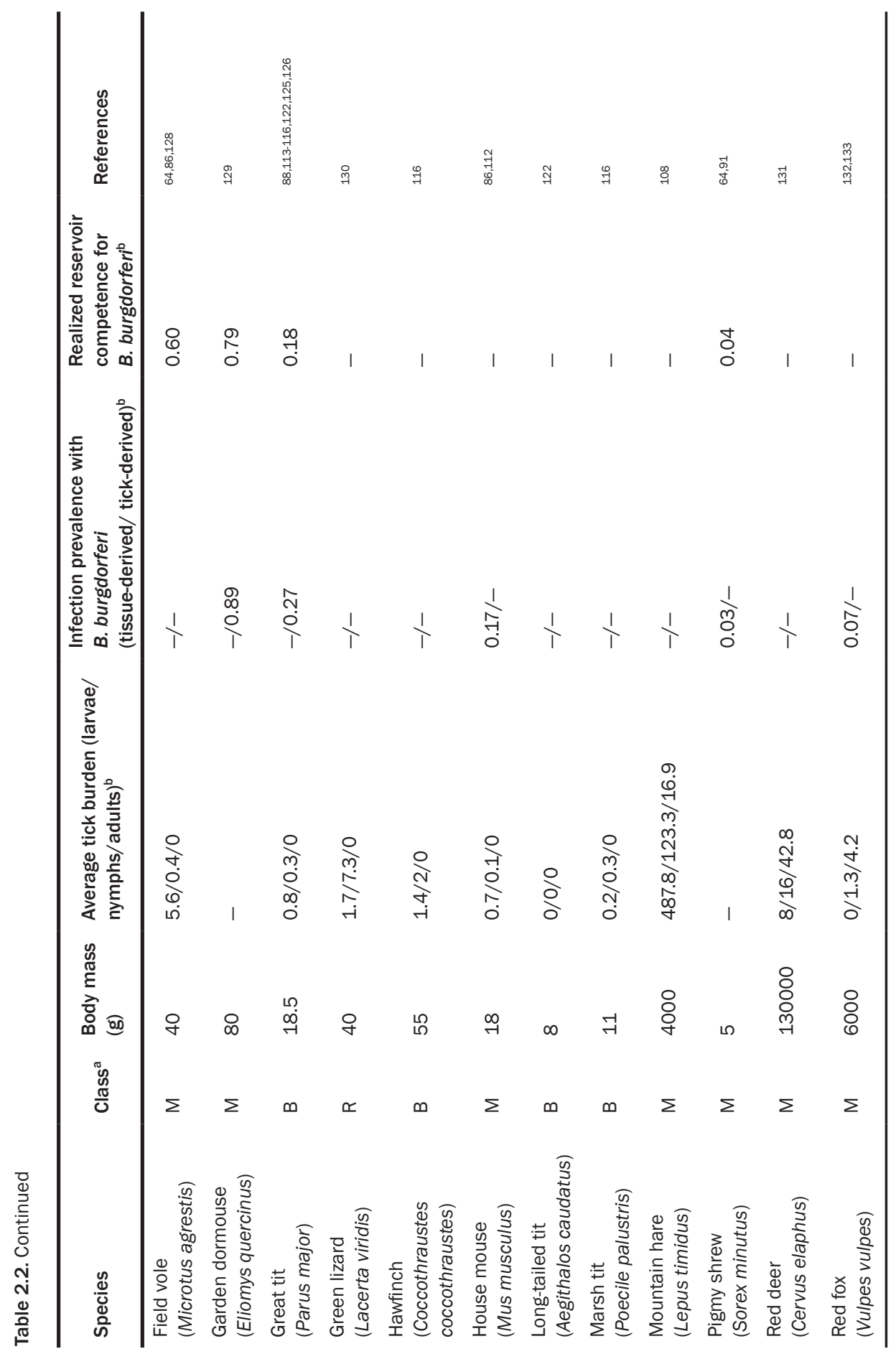




\section{4 | Chapter 2}

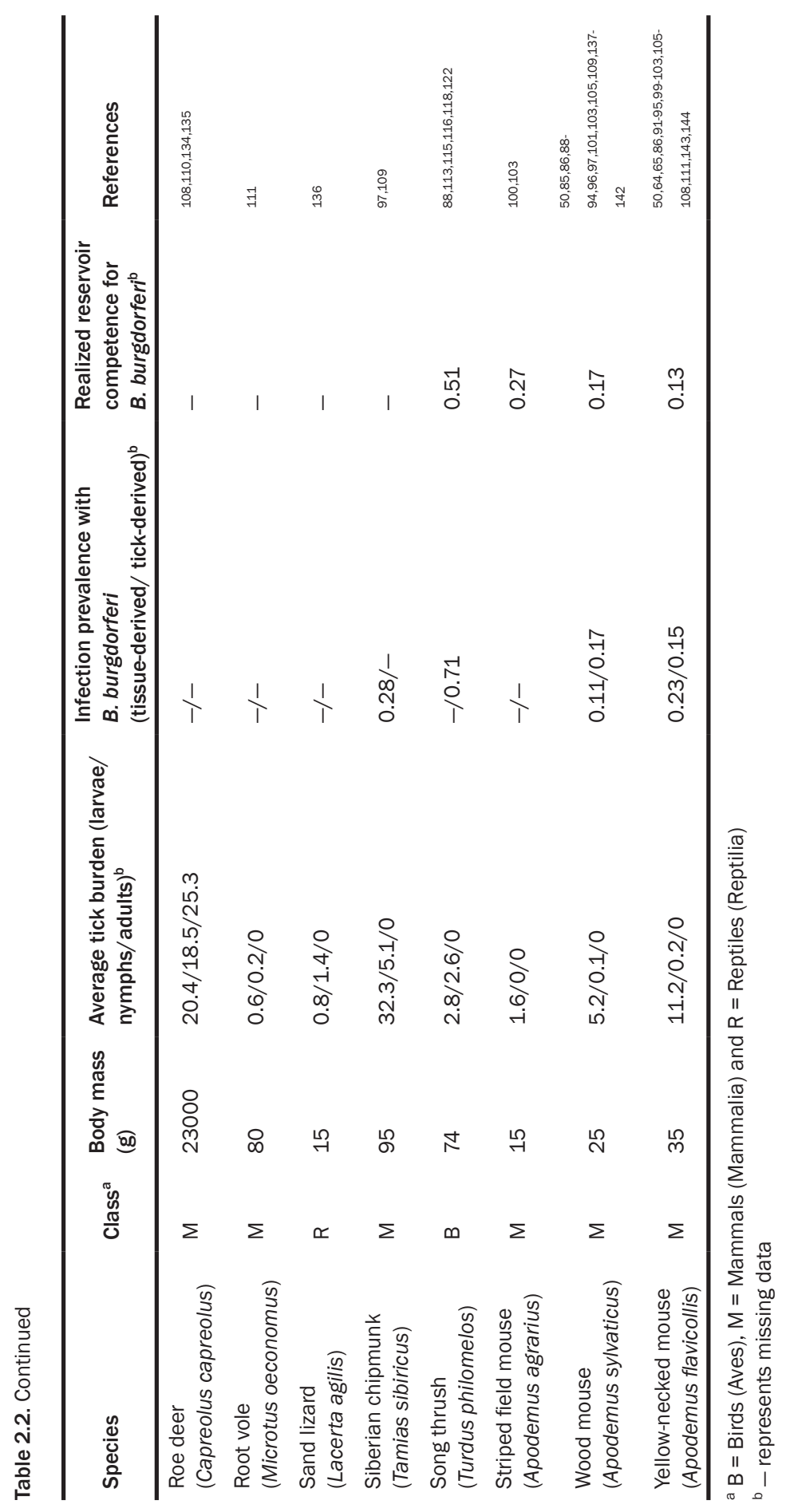




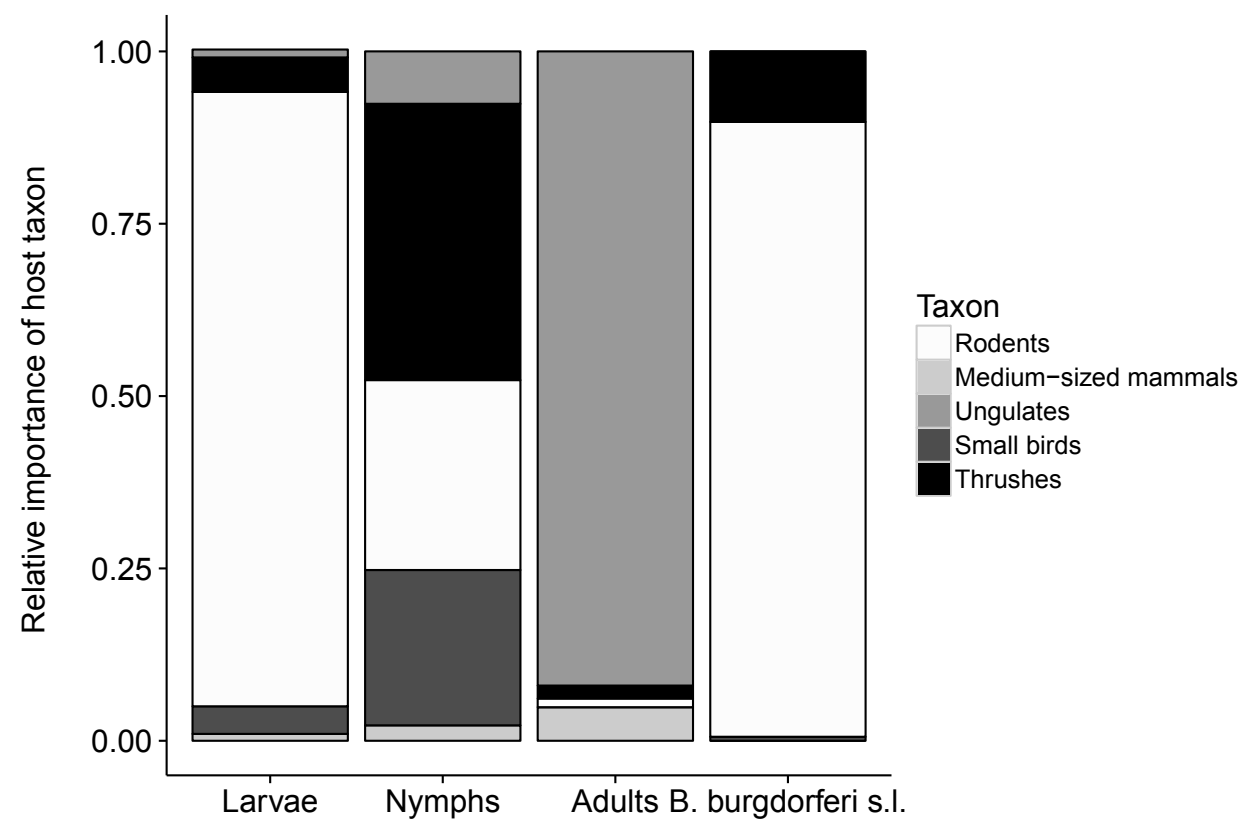

Figure 2.2. Quantification of the relative importance of different taxonomic groups of hosts feeding $I$. ricinus larvae, nymphs and adults, and infecting I. ricinus larvae with B. burgdorferi.

Relative importance of host groups for I. ricinus and B. burgdorferi The quantification of the relative importance of host species feeding I. ricinus indicated that rodents contributed most (89\%) to feeding larval I. ricinus (Figure 2.2). Although the absolute value changes with different scenarios (range: 8094\%), rodents were the most important host group feeding I. ricinus larvae in all our scenarios (Figure S2.1). Thrushes were the second most important group by feeding $5 \%$ of the larvae (range: 3-9\%), followed by smaller birds (4\%; $2-8 \%$ ).

The relative importance of host groups for nymphs differed most strongly between scenarios. Thrushes had the highest contribution to feeding nymphal $I$. ricinus (40\%; 29-49\%), while rodents (28\%; 16-43\%), small birds (23\%; 16-28\%) and ungulates (8\%; 4-14\%) were also important, depending on the scenario. Ungulates contributed most (92\%) to feeding adult I. ricinus (Figure 2.2). The absolute value of the importance of ungulates feeding adults changed per scenario (range: 85-96\%), but in all scenarios the majority of adult $I$. ricinus fed on ungulates. The second most important group were medium-sized mammals (5\%; $3-9 \%)$. 


\section{6 | Chapter 2}

The relative importance of host species for $B$. burgdorferi was calculated using only a subset of the host species in the dataset for which realized reservoir competence estimates were available (9 of the 15 species; Table 2.2). In all scenarios, rodents were the most important host group infecting larval I. ricinus with B. burgdorferi (89\%; 80-94\%), followed by thrushes (10\%; 5-18\%). However, it has to be noted that no data were available on realized reservoir competence for any of the medium-sized mammals and ungulates. For the fifteen species in our calculations, density decreased with body mass for mammals $\left(F_{1,4}=10.05\right.$, $p=0.03, \beta=-0.99)$ but not for birds $\left(F_{1,7}=0.12, p=0.74\right)$.

\section{Discussion}

Importance of host species in maintaining I. ricinus

Although $I$. ricinus is found to parasitize a large number of host species ${ }^{145}$, we found that the different stages of $I$. ricinus mainly feed on a few host species from different taxonomic groups. For birds, we found that species with higher body mass feed most I. ricinus of all stages. As host body mass is not correlated with host density in birds, it is the same large species of bird that contribute most to tick feeding by birds. In our analysis these were two thrushes, the Eurasian blackbird (Turdus merula) and Song thrush (Turdus philomelos), two abundant and widespread species in many European countries ${ }^{79}$. These species mostly forage on the ground, shifting through the litter layer looking for food ${ }^{79}$, which might be the main reason for the relatively high number of found on these species.

For mammals, which occur in the highest densities, we found a negative correlation between density and body mass, but no relationship between larval burden and body mass. Therefore, small mammals, occurring in high densities and having relatively large larval burdens, emerged from our analysis as the host group that was most important for feeding larval $I$. ricinus. This suggests that larval $I$. ricinus do not actively select for a host species, but rather feed on the hosts that are most abundant low in the vegetation where they quest ${ }^{40}$. The main studied small mammal host species were the Bank vole (Myodes glareolus), Field vole (Microtus agrestis), Wood mouse (Apodemus sylvaticus) and Yellownecked mouse (Apodemus flavicollis), all widespread and abundant species in many European countries ${ }^{80}$. 
When looking at nymphs and adults, however, there was a strong correlation between host body mass of mammals and $I$. ricinus burden. This was reflected in our analysis by the higher relative importance of medium-sized mammals and ungulates for these stages, although small mammals were still the most important mammalian host group for nymphs, due to their high densities. Ungulates were the most important host group feeding adult $I$. ricinus, which suggests that adult $I$. ricinus actively select large mammals as hosts, regardless of their relatively low densities. This might be why adult ticks quest higher in the vegetation compared to nymphs and larvae ${ }^{40}$. In our analysis of host importance the only ungulate species included was the Roe deer (Capreolus capreolus), the most widespread ungulate species in Europe ${ }^{80}$. In the absence of Roe deer, other ungulate species can serve as important hosts for adults as well.

Tick distributions on hosts are often highly over-dispersed ${ }^{8}$ and summarizing this distribution by a mean value might not result in the best parameter. Nevertheless, most papers used in our analysis did not specify any other parameters, which resulted in our use of a mean per-capita tick burden per species. We do not think that this has greatly influenced our results. The differences in mean per-capita tick burden between species are much larger than differences between studies for the same species, and most values used in our analysis are based on large sample sizes. Furthermore, the different scenarios we used in our framework showed the same general patterns, showing that these patterns are not very sensitive to changes in mean tick burden (Figure S2.1). We do, however, strongly urge for a standardized reporting system for summarizing the numbers of ticks found on hosts, for which the reporting of the number of hosts, the prevalence of infestation, the median intensity of infestation, including confidence intervals, and the exponent $k$ of the negative binomial distribution could be used ${ }^{146}$. We show that all stages of $I$. ricinus can be maintained by only a few host species that are widespread throughout Europe. This, together with a large distribution in suitable habitat and climatic conditions, explains why I. ricinus has such a wide distribution, and why it occurs in high densities in many areas with a vertebrate assemblage existing of widespread species. It also supports the hypothesis that the increase in Lyme borreliosis may be due to an increase in I. ricinus distribution and abundance ${ }^{39,61}$, following increases in range and abundance of widespread host species such as Bank vole, Blackbird and Roe deer ${ }^{147-149}$. 


\section{8 | Chapter 2}

Infection prevalence of host species with B. burgdorferi

We found that for small to medium-sized mammals and birds, the infection prevalence of host species with $B$. burgdorferi increased with their nymphal burden, with a stronger pattern for birds compared to mammals (Figure 2.1). We did not have data on infection prevalence for the largest mammals in our analysis: Red deer (Cervus elaphus) and Roe deer. Studies that were not incorporated in our selection, for reasons outlined above, show that Roe deer have high levels of antibody in their blood, and low infection prevalence in tissues ${ }^{150-152}$. These findings support the hypothesis that $B$. burgdorferi is unable to circumvent the host complement of deer ${ }^{131,153}$, which could also explain the incapability of deer to transmit Borrelia spirochaetes ${ }^{48}$. This shows that the relationship between number of nymphs feeding on a species and infection prevalence might not be linear, signifying the need for more data on infection prevalence in ungulates, other large mammals, and large bird species.

We estimated the infection prevalence of host species for B. burgdorferi using tissue-derived data as this is the best method to determine infection prevalence of animals, as not all infected animals carry ticks that can be tested ${ }^{65}$. For our analysis, we complemented the dataset with tick-derived data only for species for which tissue-derived infection prevalence was not available. The difference between estimates of both methods within species are much smaller than the differences between species (Table 2.2). Therefore, we conclude that using a combination of methods did not strongly affect our results, although our results might be underestimates because not all infected animals carry infected ticks ${ }^{154}$, and not all tissues from infected animals test positive ${ }^{96}$.

We recommend future studies to test a combination of multiple tissues and engorged ticks to get the best possible estimate of infection prevalence of hosts with B. burgdorferi. For species that are able to transmit B. burgdorferi, xenodiagnosis using $I$. ricinus larvae will further increase the accuracy of infection prevalence estimates ${ }^{137}$.

\section{Realized reservoir competence for $\mathrm{B}$. burgdorferi}

The realized reservoir competence for $B$. burgdorferi of mammals and birds $<100 \mathrm{~g}$ increases with $B$. burgdorferi infection prevalence of the species (Figure 2.1). For small mammals we show a negative correlation between body mass and realized reservoir competence when we correct for differences in infection prevalence. It is hypothesized that smaller, short lived, species invest less in their 
immune system than larger, longer lived, species ${ }^{68}$. However, this hypothesis is debated for differences at a lower taxonomic level ${ }^{155}$, so it might not be the most likely explanation for the correlation we found for rodents and shrews. We think it is more likely that $B$. burgdorferi has adapted to the species it most often encounters. This idea was supported by a positive correlation between density and realized reservoir competence of mammalian hosts for several tick-borne pathogens in North America ${ }^{30}$. We found a negative correlation between host body mass and density for mammals in our analysis, so the found correlation of realized reservoir competence with body mass could actually be the result of differences in densities of the different species.

For small songbirds we found a positive correlation between body mass and realized reservoir competence when we correct for differences in infection prevalence. This correlation could be caused by the two largest species (Blackbird and Song thrush) which have a far higher realized reservoir competence than the others. These species also have the highest nymphal burden and infection prevalence, showing that these are the bird species that $B$. burgdorferi most often encounters. Therefore, the adaptation of $B$. burgdorferi to the species it most often encounters could explain the different correlations with body mass we found for small mammals and birds.

\section{Importance of host species in infecting larvae with B. burgdorferi}

In our analysis, rodents, which occur in high densities and have relatively large larval burdens, but relatively low realized reservoir competence, had the highest relative importance for infecting larvae with $B$. burgdorferi. Thrushes were the second most important group, having intermediate densities and larval burdens, but a very high realized reservoir competence. This indicates that the number of larvae feeding on a host species and its density are more important than the realized reservoir competence of that host species in determining the contribution of a host species to infect larvae. Furthermore, it suggests that the prevalence of different $B$. burgdorferi genospecies in questing ticks is mainly dependent on the distribution of larvae over rodents and thrushes.

The feeding pattern of ticks could explain why, in most areas in Europe, $B$. afzelii is the most common genospecies found in questing nymphs ${ }^{41}$. We found that $89 \%$ of the infected larvae in our analysis had fed on rodents. This should result in a large percentage of $B$. afzelii-infected nymphs as $B$. afzelii is transmitted by small mammals ${ }^{65}$. Thrushes fed $10 \%$ of the infected larvae, which 


\section{0 | Chapter 2}

could explain the relatively low percentages of $B$. garinii and $B$. valaisiana in fieldderived nymphs ${ }^{156}$.

Borrelia spp. transmission maintenance

The large difference in infection prevalence between small mammals and birds together with their large differences in relative importance for B. burgdorferi suggest that there are two distinct mechanisms behind the maintenance of small mammal-transmitted Borrelia spp. and bird-transmitted Borrelia spp. ${ }^{48}$. Because small mammals have low nymphal burdens, their infection prevalence with $B$. burgdorferi is relatively low (Table 2.2). However, because they feed such a large proportion of the larvae, even a small infection prevalence of the host species can result in a high density of infected nymphs with small mammaltransmitted Borrelia spp. like B. afzelii. This high density of nymphs infected with small mammal-transmitted Borrelia spp. results in a sufficiently-large number of infected nymphs to, in turn, infect small mammals in spite of their low nymphal burdens.

Bird-transmitted Borrelia spp., like B. garinii and B. valaisiana, on the other hand, seem to be dependent on high infection prevalence of their host species due to relatively high nymphal and adult burdens (Table 2.2). Therefore, even with a low larval burden and intermediate host density, sufficient numbers of infected nymphs are produced to infect birds, which completes the maintenance cycle for bird-transmitted Borrelia spp. However, this strategy is probably not only restricted to bird-transmitted Borrelia spp. Borrelia spielmanii is a candidate for a similar maintenance strategy in mammals as it is often found with low prevalence in questing ticks, but with high prevalence in one of its principal hosts, the Garden dormouse (Eliomys quercinus) ${ }^{129}$.

These differences in maintenance strategies could indicate that less common Borrelia spp., or other tick-borne pathogens with low infection prevalence in questing nymphs, might be maintained by host species with high nymphal or adult burdens ${ }^{30}$. Also it shows that $B$. burgdorferi can specialize either on host species that occur in high densities, or on host species that feed large numbers of ticks, with the exception of larger bodied mammalian species such as deer.

\section{Host species diversity}

Ostfeld and Keesing ${ }^{157}$ proposed a dilution effect of host species diversity on Lyme borreliosis risk. This hypothesis has been highly debated, especially in the 
context of ticks from the I. ricinus complex and Lyme borreliosis ${ }^{158,159}$. Although our analysis did not examine the effect of differences in species richness, our methods could be used to quantify the relative contributions of different species in different assemblages, as long as differences in tick burden and density are accounted for. Our results suggest that few, but widespread vertebrate species feed most of the ticks in European forests. Therefore, community related factors influencing either the densities or tick burdens of these species can have an effect on the outcomes of the calculations. For example, the presence of predators could have effects on the densities or tick burdens of rodents, which may affect the number of $B$. burgdorferi infected ticks in the vegetation ${ }^{160}$.

\section{Limitations of the data}

There is little information available on the infection prevalence with and realized reservoir competence for the different genospecies of $B$. burgdorferi such as B. afzelii, B. bavariensis, B. burgdorferi s.s., B. garinii, B. Iusitaniae, B. spielmanii and $B$. valaisiana. For some widespread host species such as European hedgehog (Erinaceuseuropaeus), European hare(Lepuseuropaeus), and Field vole(Microtus agrestis) there were no data available at the genospecies level that would satisfy our selection criteria. Furthermore, for some widespread host species, such as Eurasian badger (Meles meles), Eurasian jay (Garrulus glandarius), Eurasian pygmy shrew (Sorex minutus), Eurasian red squirrel (Sciurus vulgaris), European pine marten (Martes martes), Great spotted woodpecker (Dendrocopos major), Wild boar (Sus scrofa) and Wood pigeon (Columba palumbus), either very little, or no data at all were available. For example, the two studies ${ }^{161,162}$ that lead the current opinion that Eurasian red squirrels are important hosts in transmitting and maintaining B. burgdorferi s.s. in Europe had either a very low sample size ${ }^{161}$, or the animals were collected throughout the year in different habitat types, without specifying infection prevalence per season/habitat type ${ }^{162}$. Therefore, we stress that data on tick stages and genospecies-specific infection should be collected from these host species during the active period of $I$. ricinus, in natural habitats, in order to be able to analyse a more complete host assemblage. This would also enable the analysis of the relationships between host body mass, density, tick burdens, infection prevalence and realized reservoir competence for the different genospecies. 


\section{2 | Chapter 2}

\section{Conclusion}

Our analysis suggests that a few vertebrate species that are widespread in Europe are the most important host species feeding I. ricinus and transmitting B. burgdorferi. We demonstrate that vertebrate species with a higher body mass have a higher I. ricinus burden, that host species with higher tick burdens are more likely to be infected with $B$. burgdorferi and that species that are more often infected with $B$. burgdorferi also transmit the infection more often to larval I. ricinus. These patterns suggest that $B$. burgdorferi adapts to the species it most often encounters.

To our knowledge, this review is the first to quantify the relative importance of host species for the different stages of $I$. ricinus, and our calculations support the widely held idea that small rodents are the most important hosts in feeding larval I. ricinus, that birds and rodents feed most of the nymphs, and that ungulates are the main hosts for adult $I$. ricinus ${ }^{36,62}$. We found that rodents and thrushes contribute most to the pool of $B$. burgdorferi infected nymphs. We suggest two different maintenance strategies for $B$. burgdorferi, which are correlated to high host densities or high infection prevalence in the hosts. These might explain how some tick-borne pathogens can be maintained with very low prevalence in questing ticks. We show that using a simple framework and a systematic data search can be used to calculate the relative importance of host species for tick species, and tick-borne pathogens, which can be used in research on other tick species and other tick-borne pathogens. These results can aid selection of host species to target for intervention strategies ${ }^{76}$.

Rodents, thrushes and deer, that are the most important host groups feeding I. ricinus and infecting I. ricinus larvae with $B$. burgdorferi, have increased in distribution and abundance in recent decades due to changes in land use and forest management ${ }^{147-149}$, which could be the main driver behind increased disease incidence with tick-borne diseases in Europe. 


\section{Few Vertebrate Species Dominate the B. burgdorferi Life Cycle | 43}

\section{Appendix to Chapter 2}

\section{Appendix 2.1: Search string and part of the selection procedure}

\section{The search string used was:}

(Ixodes ricinus OR Borrelia burgdorferi) AND (distribution OR presence OR occurrence OR reported OR incidence OR prevalence OR spread OR dispersion OR detection OR diagnosis OR isolation OR counts OR burden OR infestation) AND (Accipiter OR Acrocephalus OR Alauda OR Alca OR Alces OR Allactaga OR Alopex OR Anas OR Anser OR Anthus OR Apodemus OR Aquila OR Arvicola OR Asio OR Athene OR Bison OR Bomyicilla OR Bonasa OR Bos OR Bubalus OR Bubo OR Buteo OR Canis OR Capra OR Capreolus OR Caprimulgus OR Carduelis OR Castor OR Cepphus OR Certhia OR Cervus OR Charadrius OR Chionomys OR Chroicocephalus OR Clethrionomys OR Coccothraustes OR Columba OR Corvus OR Coturnix OR Crex OR Cricetulus OR Cricetus OR Crocidura OR Cuculus OR Dama OR Darevskia OR Dendrocopos OR Dryomys OR Eliomys OR Emberiza OR Equus OR Erinaceus OR Erithacus OR Eutamias OR Falco OR Felis OR Ficedula OR Francolinus OR Fringilla OR Fulica OR Gallinago OR Garrulus OR Genetta OR Glis OR Hemiechinus OR Hemorrhois OR Hippolais OR Hirundo OR JacuIus OR Jynx OR Lacerta OR Lagopus OR Lagurus OR Lanius OR Larus OR Lemmus OR Lepus OR Locustella OR Loxia OR Luscinia OR Martes OR Meles OR Mesocricetus OR Micromys OR Microtus OR Miliaria OR Milvus OR Motacilla OR Mus OR Muscardinus OR Muscicapa OR Mustela OR Myocastor OR Myodes OR Neomys OR Netta OR Numenius OR Nyctereutes OR Ochotona OR Oenanthe OR Ondatra OR Oryctolagus OR Ovis OR Parus OR Passer OR Perdix OR Pernis OR Phalacrocorax OR Phasianus OR Philomachus OR Phoenicurus OR Phylloscopus OR Pica OR Picoides OR Picus OR Pluvialis OR Podarcis OR Podiceps OR Porzana OR Prunella OR Pyrrhula OR Rattus OR Regulus OR Rhinolophus OR Riparia OR Rupicapra OR Saxicola OR Sciurus OR Scolopax OR Sicista OR Sitta OR Sorex OR Spalax OR Spermophilus OR Streptopelia OR Strix OR Sturnus OR Sus OR Sylvia OR Talpa OR Tamias OR Testudo OR Tetrao OR Timon OR Troglodytes OR Turdus OR Tyto OR Upupa OR Ursus OR Vanellus OR Vipera OR Vulpes OR Zootoca)

Genus nomenclature has been stable in Europe since 1945 and for those genus names that did change, we used both the old and the new genus name. As restrictions in PubMed we looked only for the publications for which full texts were available, and that pertained to animal species. For the publications that seemed to contain relevant data judged on their title, keywords and abstract, the full text version was downloaded. Their bibliographies were screened for citations not identified in the initial step of the literature search to get as complete a database as possible. 


\section{4 | Chapter 2}

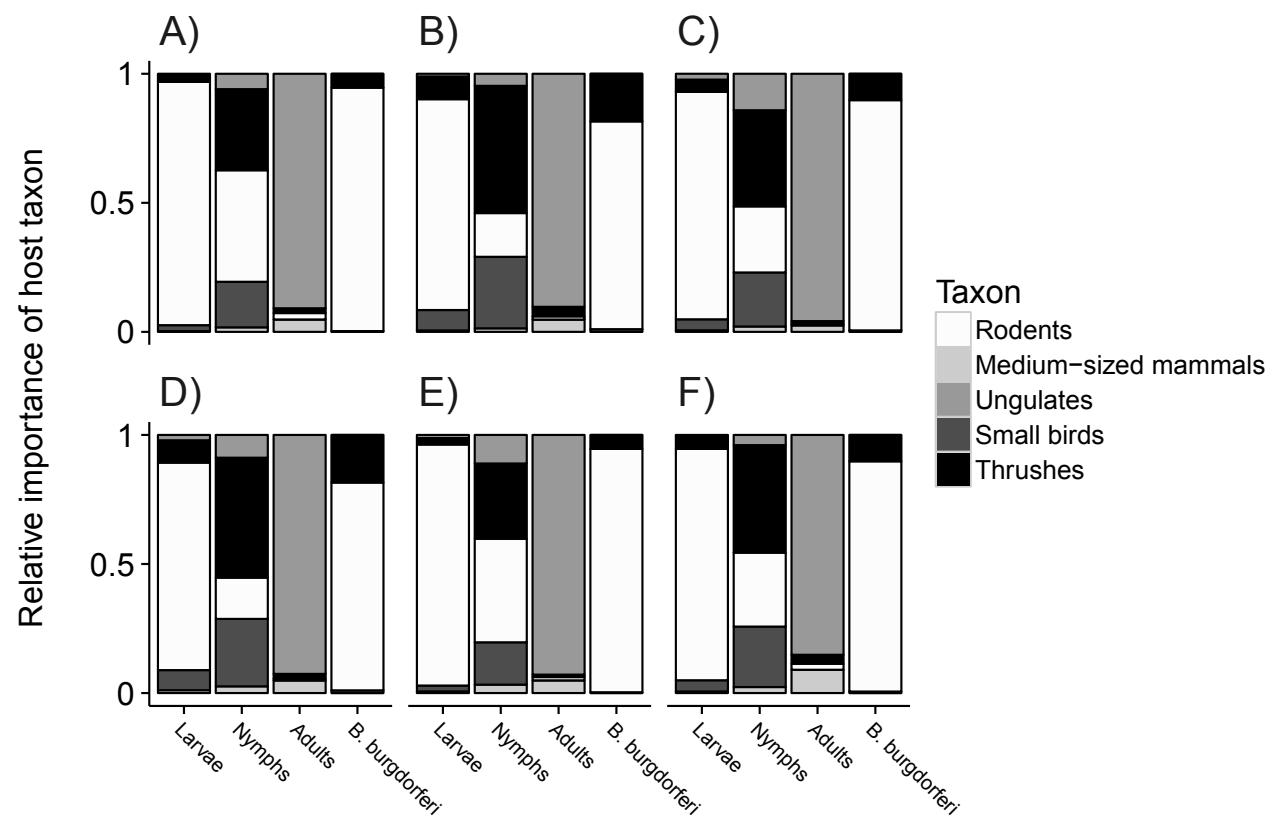

Figure S2.1. Different scenarios for the quantification of the relative importance of different host groups feeding I. ricinus larvae, nymphs and adults, and infecting I. ricinus larvae with B. burgdorferi. A) Twofold increase in rodent per-capita tick burden for all stages. B) Twofold increase in bird per-capita tick burden for all stages. C) Twofold increase in ungulate per-capita tick burden for all stages. D) Twofold decrease in rodent per-capita tick burden for all stages. E) Twofold decrease in bird per-capita tick burden for all stages. F) Twofold decrease in ungulate per-capita tick burden for all stages. In each model, all other parameters were held constant. 



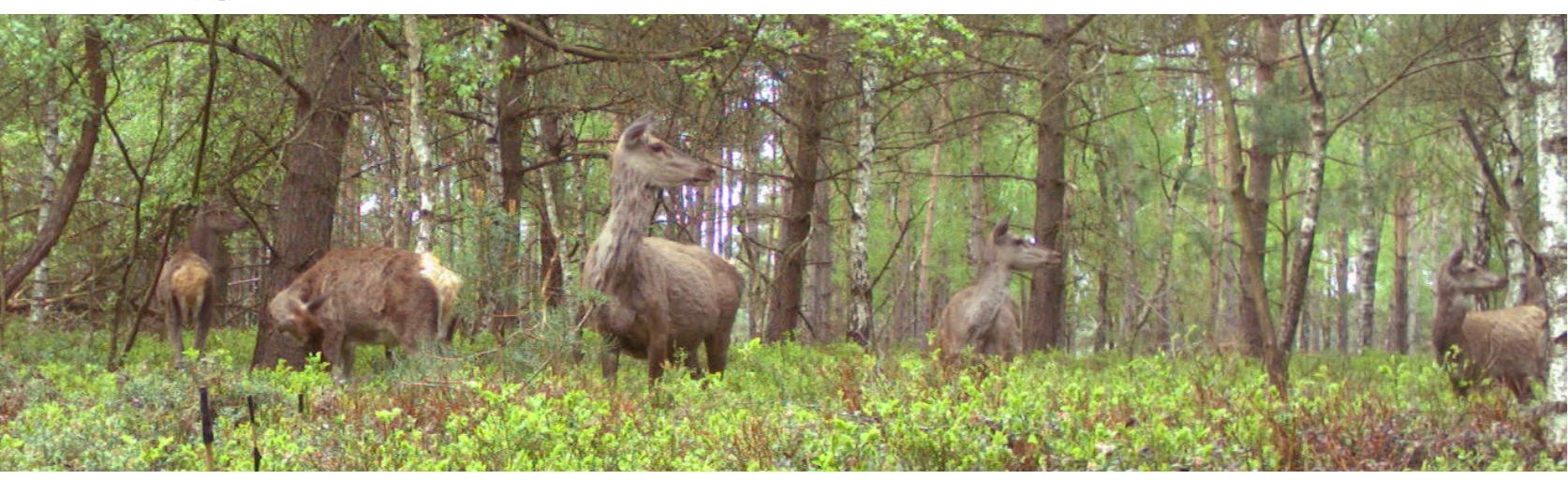

flashes without thunder are flaring night and day what they are detecting is sometimes hard to say

distance, size they matter the kind of vegetation too too much leaves can scatter so how to tell what's true Ronald Hofmeester 


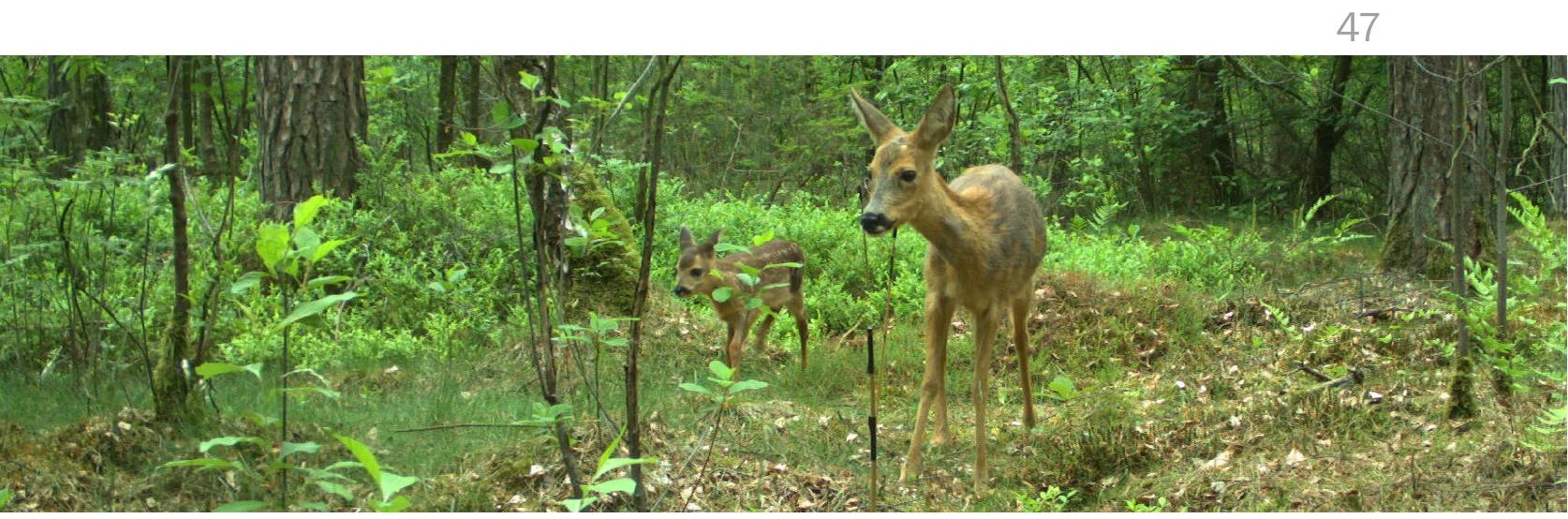

T.R. Hofmeester, J.M. RowclifFe \& P.A. Jansen Remote Sensing in Ecology and Conservation (Online First)

\section{A Simple Method for Estimating the Effective Detection Distance of Camera Traps}

Estimates of animal abundance are essential for understanding animal ecology. Camera traps can be used to estimate the abundance of terrestrial mammals, including elusive species, provided that the sensitivity of the sensor, estimated as the effective detection distance (EDD), is quantified. Here, we show how the EDD can be inferred directly from camera-trap images by placing markers at known distances along the midline of the camera field of view, and then fitting distance sampling functions to the frequency of animal passage between markers. EDD estimates derived from simulated passages using binned detection distances approximated those obtained from continuous detection distance measurements if at least five intervals were used over the maximum detection distance. A field test of the method in two forest types with contrasting vegetation density, with five markers at $2.5 \mathrm{~m}$ intervals, produced credible EDD estimates for thirteen forest-dwelling mammals. EDD estimates were positively correlated with species body mass, and shorter for the denser vegetation, as expected. Our findings suggest that this simple method can produce reliable estimates of EDD. These estimates can be used to correct photographic capture rates for differences in sampling effort resulting from differences in sensor sensitivity between species or habitats. Simplifying the estimation of EDD will result in less biased indices of relative abundance, and will also facilitate the use of camera trap data for estimating animal density. 


\section{8 | Chapter 3}

\section{Introduction}

Estimation of animal abundance is essential for understanding animal ecology and for wildlife management and conservation. However, for many species (e.g., forest-dwelling mammals, elusive carnivores) conventional techniques such as capture-mark-recapture or line transect counts are difficult and timeconsuming ${ }^{163}$. Cameras with passive infrared (PIR) sensors, commonly referred to as camera traps or trail cameras, can detect rare, cryptic and elusive animals and are increasingly used to detect and monitor wild life worldwide ${ }^{52}$. PIR sensors detect a difference in heat-and-motion between subjects and the environment and trigger the camera if the difference exceeds a pre-set threshold ${ }^{164}$. Thus, animals that are warmer than their surroundings will trigger the camera when passing within the range of the sensor and are photographed or filmed.

When animals can be individually identified, camera traps can be used to perform conventional capture-mark-recapture using the capture rate of known individuals ${ }^{165}$. For animals that cannot be distinguished individually, camera traps are often used to derive relative abundance estimates, reasoning that photographic capture rates - the number of visits recorded per unit time - of a species will be proportional to its abundance ${ }^{166}$. However, the use of capture rates as relative abundance indices has received criticism, as capture rates are influenced by other factors besides animal abundance, which can lead to important biases (e.g., Sollmann et al. ${ }^{167}$ ). Some of these factors are related to non-random movement of the focal species, such as the preferred use of trails. However, most factors that bias capture rates are related to the PIR sensor of the camera trap: camera type, camera placement, animal size, vegetation density, temperature and relative humidity all influence the effective range of the sensor ${ }^{54,168}$. Some of these factors can be dealt with using adequate sampling design, for example, using the same camera model and thus the exact same PIR sensors ${ }^{164}$, the same sampling period, similar climate, and random sampling points ${ }^{167,168}$. However, when the goal is to compare different species, or the same species in different habitat types, biases may remain a problem.

Although larger animals omit less heat per unit of mass than small animals, they still omit a larger absolute amount of heat (thermal infrared) than do small animals ${ }^{169}$. Therefore, the PIR sensor of a camera trap is more sensitive to large animals than to smaller animals ${ }^{54}$. Furthermore, most PIR sensors are more sensitive in the middle of the field of view (FOV) than at the edges ${ }^{164}$, which 
also results in differences in detectability between large and small species ${ }^{54}$. The resulting differences in sampling area can be corrected for by estimating the effective detection distance (EDD) and angle for each species ${ }^{54}$. The EDD is the distance at which the number of animals detected further away equals the number of animals missed nearer by. The estimation of EDD and angle are essential for using the Random Encounter Model (REM) ${ }^{170}$, which estimates animal densities by correcting photographic capture rates for detection bias and species' day range. Rowcliffe et al. ${ }^{54}$ measured distances and angles by tracking the movement path of animals through the FOV on site, and measuring the distance and angle from the camera at first detection, which required a substantial investment of field time. This method was recently simplified by the use of a photograph of a grid of markers taken after each camera deployment to estimate detection distance and angle using image processing software ${ }^{171}$.

Here, we present an further simplified method to estimate the EDD directly from camera trap images. The principle is to establish markers at known distances along the midline of the FOV, record the frequency of animal passage between markers, and then fit distance sampling functions to distance distributions to estimate EDD. To determine whether this approach was effective, we compared it with the conventional method described by Rowcliffe et al. ${ }^{54}$ using simulated data. Then, in a field study, we tested whether EDD estimates derived with the line marker method 1) increased with body mass, as larger animals emit more infrared radiation; and 2) were shorter in denser vegetation, as vegetation affects transmission of infrared radiation.

\section{Methods}

We approached detection of animals by camera traps using distance sampling detection models (sensu Rowcliffe et al. ${ }^{54}$ ). The trigger threshold of the PIR sensor of a camera trap is more easily exceeded by a warm-blooded animal walking close to the sensor than by an animal walking further away from the sensor. When we assume that an animal walking against the camera (distance of zero) is always detected, we can model the probability of being detected as a standard monotonically declining detection model. When the distance from the camera to each animal triggering the camera is known, these distances can be used as input for the detection model. While exact distances are hard to obtain, distance classes are easily obtained from the images if intervals are marked in the FOV, 


\section{0 | Chapter 3}

and using distance classes is general practice in distance sampling ${ }^{172}$. Distance classes can be realized by establishing markers at known distances along lines running away from the camera when the camera is set up. As it is not practical to place such lines along multiple angles in the full FOV, we reasoned that the distance at which an animal triggers the camera and the distance at which an animal walks through the middle of the FOV are correlated. If this assumption holds, detection distance can be estimated by placing a line of markers in the middle of the FOV. We tested this assumption using a simulation (see below).

Distance data are obtained during annotation of the photographs, as the analyst records between which markers photographed animals pass, assigning each passage to a distance interval. All triggers of animals that do not cross the midline are ignored. The distribution of passages over the different distance categories is then used to fit a detection probability function from which EDD can be estimated using standard techniques for estimating effective strip width (line model) or effective detection radius (point model) ${ }^{173}$. Where a line model assumes that the histogram of the number of detections over the different distance categories follows a distribution similar to the detection probability, a point model assumes that the distribution of the histogram is similar to the detection probability multiplied by the distance, correcting for the increase of detection area with distance. In our case, the detection along a centre line may be best described by a line model, while the true detection by the sensor may be better described by a point model ${ }^{54}$. Because the reduction of a cone-shaped detection area to a line might not result in a perfect line detection, we considered both models in our analysis. The fitting of detection probability functions to distance data can be done with several software packages such as DISTANCE ${ }^{174}$ or the R package mrds $^{175}$.

Following Rowcliffe et al. ${ }^{54}$ we use two different detection probability models: A half-normal model and a hazard-rate model, respectively:

$$
\begin{aligned}
& g(x)=\exp \left(\frac{-x^{2}}{2 \cdot \alpha^{2}}\right) \\
& g(x)=1-\exp \left(-\left(\frac{x}{\alpha}\right)^{-\gamma}\right)
\end{aligned}
$$

Equation 3.1

Equation 3.2

where $g(x)$ is the detection probability at distance $x, \alpha$ defines the width of the function, and $y$ the shape of the hazard-rate function. These detection functions 
can also be expanded using covariates, such as body mass, habitat type or season ${ }^{54}$. We fitted both simple distance-sampling models without covariates on single species in each habitat, and multiple-covariate distance-sampling models, including body mass and habitat as covariates (see below) ${ }^{176}$. We selected the best-performing models based on AIC values as estimates of model quality ${ }^{172}$.

\section{Simulation}

To determine whether EDD estimated using grouped distances along the centre line reflects EDD as estimated by the baseline approach described by Rowcliffe et al. ${ }^{54}$ using direct radial distances, we bootstrapped simulated samples, and explored the effect of varying the number of intervals used. For each of 1000 samples, we defined 100 random positions of first detection (point of trigger) by drawing radii from a hazard-rate distribution and angles from a half-normal distribution. We then assigned each position a random direction of travel (uniform in 0-2 $\pi$ ), and calculated the distance at which its trajectory crossed the camera's line of sight (centre line distance). To define trajectories that could be observed to cross the camera's line of sight, we retained those that crossed the centre line in front of the camera, within the maximum trigger position distance, and after the point of trigger.

For each sample, we fitted a point detection function model to the direct trigger distance data to provide baseline expected EDD, using previously published methods ${ }^{54}$. For comparable centre line models, we fitted both point and line models with data either binned into 2, 3, 4, 5 or 10 equal distance intervals, or un-binned using a grouped likelihood for the binned models ${ }^{173}$. In all cases we used hazard-rate models with no expansion terms. We tested with different numbers of distance intervals, as to assess what number of markers should be used in the field to obtain reliable EDD estimates. Here we did not use expansion terms, as to enable a more robust estimation of EDD with low sample size.

\section{Field test}

We field tested the method by surveying mammals in forests that had contrasting densities of understory vegetation: four 1-ha plots with Scots pine (Pinus sylvestris) and a thick undergrowth of blueberry (Vaccinium myrtillus; dense understory), and four with Pedunculate oak (Quercus robur) with a sparse undergrowth of ferns (Broad buckler-fern (Dryopteris dilatata) or Bracken (Pteridium aquilinum); open understory), scattered across the Netherlands 


\section{2 | Chapter 3}

(Table S3.1). Within each plot, we deployed two camera traps (HC500, RECONYX Inc., Holmen, WI, U.S.A.) for four weeks, at two random locations $>30 \mathrm{~m}$ apart. These cameras were moved to two new locations every four weeks until 14-18 different locations were sampled. Cameras were deployed on the tree nearest to a computer-generated random point, $40 \mathrm{~cm}$ above the ground facing north, and aimed parallel to the ground. If necessary, we pruned the vegetation that blocked the view of the camera in a strip of $1 \mathrm{~m}$ broad and $5 \mathrm{~m}$ long in front of the camera. Vegetation was cut at $20-30 \mathrm{~cm}$ height to reduce false triggers due to moving vegetation which is standard practice in camera-trapping studies ${ }^{177}$. Cameras were set to take a series of ten photographs when triggered, and to be available for re-triggering without delay, so that movement between the markers would be recorded. Markers (bamboo sticks of $60 \mathrm{~cm}$ length) were placed in front of the camera, at intervals of $2.5 \mathrm{~m}$, based on the maximum distance at which the cameras could detect a human $(15 \mathrm{~m})$. The markers were topped with two strips of black tape to increase visibility of the sticks in the photographs (Figure 3.1). We placed the sticks slightly out of line, to ensure that they were all visible in the pictures. Depending on visibility through the vegetation, we used three to five markers, resulting in four to six distance intervals.

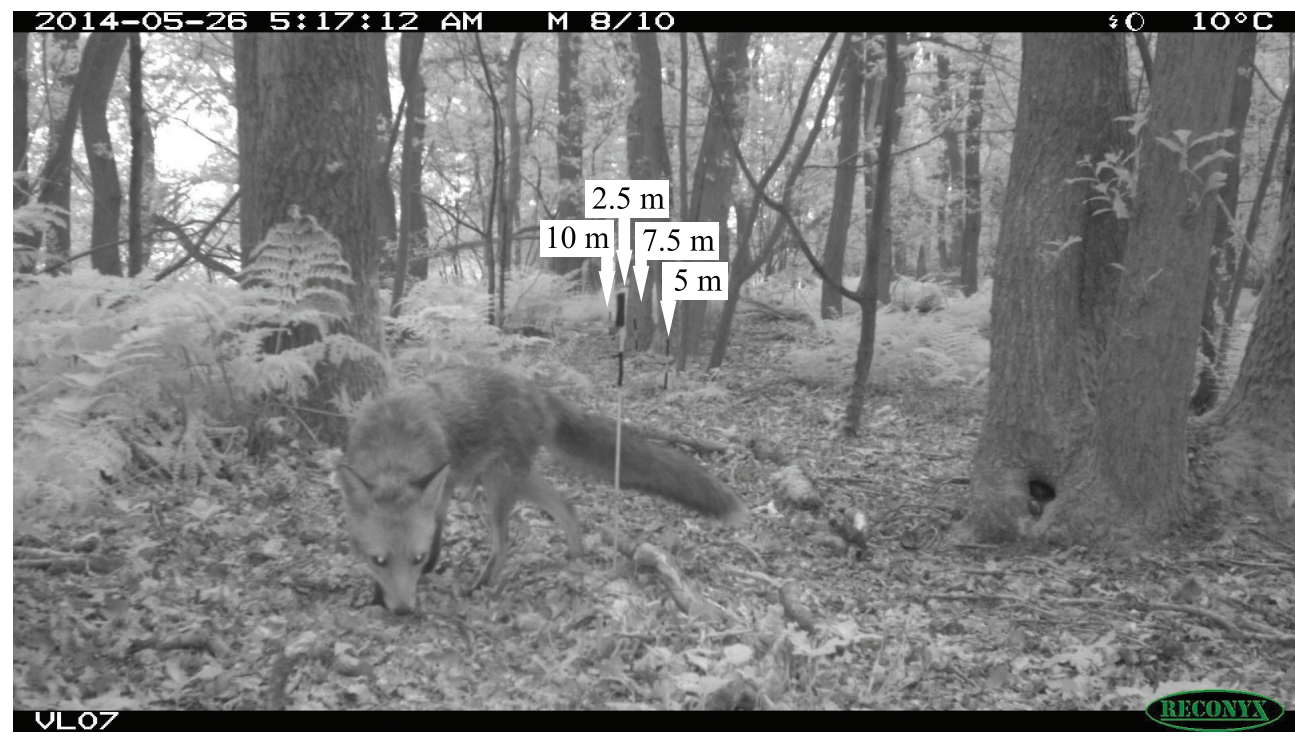

Figure 3.1. Camera trap photograph with a transect of markers at $2.5 \mathrm{~m}, 5 \mathrm{~m}, 7.5 \mathrm{~m}$ and $10 \mathrm{~m}$, and a passing Red fox (Vulpes vulpes). 
Photographs were managed and annotated using a custom-made photoprocessing tool called 'Agouti' (cf. Kays et al. ${ }^{178}$ ). All photographs were automatically grouped into sequences if less than five minutes passed between triggers, stored as separate sequences if separated more than fifteen minutes, and otherwise checked manually to determine if it was the same or a different passage that triggered the camera. For each sequence, we noted the species and separated or combined sequences if the automatic procedure grouped or split passages from the same individual or group of individuals. Furthermore, we noted, for each individual animal, if it crossed the midline of the FOV, and if so, through which interval they passed. If the animals passed just behind the last marker, we noted it as passing in an interval of $2.5 \mathrm{~m}$ behind the last marker. There were no animals that triggered the camera and passed the line $>2.5 \mathrm{~m}$ past the last marker.

All analyses were done in R 3.2.31. We used a two-step approach, where we first estimated EDD for each species - habitat combination of which we had at least 20 distance measurements (Table 3.1), using models without covariates as implemented in the mrds package version 2.1.14 ${ }^{175}$. We estimated EDD using point models, as these gave the best fit in our simulation (see results). EDD was estimated as:

$$
\mathrm{EDD}=\mathrm{w} \cdot \sqrt{\mathrm{Pa}}
$$

Equation 3.3

where $w$ is the truncation distance, and $\mathrm{Pa}$ is the expected probability of detection for an animal within distance $w$ from the camera ${ }^{172}$, which is given as output of the ddf function in the mrds package. We used the furthest distance at which an animal was detected as the truncation distance.

A single body mass estimate per species was taken from the PanTHERIA database ${ }^{179}$. As the exact relationship between being detected by the camera and body mass was not known, we tested different transformations of body mass (square root, cube root and $\log _{10}$ ). The relationship between species-specific EDD estimates and transformed body mass was modelled with least-squares regression for each habitat. We used transformed parameters in a least-squares regression to be able to use the outcome as a linear covariate in the distancesampling model. Secondly, we estimated EDD using multiple-covariate distance sampling ${ }^{172}$ with transformed body mass and habitat as covariates. Both 


\section{4 | Chapter 3}

Table 3.1. Body mass, number of distance measurements obtained, and total number of detections per habitat type for thirteen forest mammal species that were detected during this study.

\begin{tabular}{|c|c|c|c|}
\hline \multirow[t]{2}{*}{ Species $^{\mathrm{a}}$} & \multirow{2}{*}{$\begin{array}{l}\text { Body mass } \\
(\mathrm{kg})^{\mathrm{b}}\end{array}$} & \multicolumn{2}{|c|}{$\begin{array}{l}\text { Number of distance measurements / } \\
\text { Total number of detections }\end{array}$} \\
\hline & & Dense understory ${ }^{\mathrm{c}}$ & Open understory ${ }^{\mathrm{C}}$ \\
\hline Eurasian red squirrel Sciurus vulgaris (squirrel) & 0.3 & - & $7 / 9$ \\
\hline $\begin{array}{l}\text { European hedgehog Erinaceus europaeus } \\
\text { (hedgehog) }\end{array}$ & 0.8 & - & $2 / 8$ \\
\hline Polecat Mustela putorius (polecat) & 1.0 & - & $4 / 5$ \\
\hline $\begin{array}{l}\text { European pine marten Martes martes } \\
\text { (pine marten) }\end{array}$ & 1.3 & $26 / 33$ & $32 / 41$ \\
\hline Stone marten Martes foina (stone marten) & 1.7 & - & $9 / 9$ \\
\hline Feral cat Felis catus (cat) & 2.9 & - & $21 / 24$ \\
\hline European hare Lepus europaeus (hare) & 3.8 & $2 / 4$ & $148 / 199$ \\
\hline Red fox Vulpes vulpes (fox) & 4.8 & $34 / 39$ & $103 / 132$ \\
\hline European badger Meles meles (badger) & 11.9 & $14 / 18$ & $54 / 68$ \\
\hline Roe deer Capreolus capreolus (roe deer) & 22.5 & $83 / 108$ & $552 / 763$ \\
\hline Fallow deer Dama dama (fallow deer) & 57.2 & $108 / 131$ & $3 / 3$ \\
\hline Wild boar Sus scrofa (wild boar) & 84.5 & $551 / 633$ & - \\
\hline Red deer Cervus elaphus (red deer) & 240.9 & $390 / 460$ & - \\
\hline
\end{tabular}

a Abbreviated common names in brackets are used hereafter.

${ }^{\mathrm{b}}$ Body mass values as given in the PanTHERIA database ${ }^{179}$.

${ }^{c}$ Detection distance sample sizes above 20 are printed in bold

- represents combinations of species and habitat type that were not observed.

covariates were modelled as additive to the scale parameter of the detection function. For this analysis we used all distance estimates, from all species in both habitats. The advantage of using a model including covariates, is that all distance measurements for all species can be used. This enables the estimation of EDD for species for which we had too few measurements for fitting singlespecies models (Table 3.1).

\section{Results}

\section{Simulation}

We found that point models applied to centre line data (Figure 3.2) matched predetermined EDD fairly well (off $5 \%$ on average), whereas line models (Figure S3.1) produced overestimates (by about 34\% on average). Expected EDD was 
somewhat sensitive to the number of intervals used in centre line models at very small interval numbers, with higher estimates on average using only two intervals, but no change in the expectation above three intervals. With three or four intervals there was a tendency for models to fit poorly, giving extremely low EDD estimates on occasion. With five or more intervals the result was effectively indistinguishable from the un-binned analysis.

\section{Field test}

We recorded thirteen mammal species, nine of which had more than 20 distance measurements in one or both habitats (Table 3.1). The percentage of animals that walked across the midline of the FOV, i.e. yielded distance measurements, was higher in the areas with a dense understory (85\%) than in areas with a more open understory (74\%), and differed slightly between species (Table 3.1). Using the distance-interval measurements, a half-normal detection probability function gave the best fit for most species-habitat combinations except Fallow deer and Red fox in pine forest and European badger in oak forest, where a hazard-rate function performed marginally better (Table 3.2). Estimated EDD increased significantly with body mass, as predicted, where a $\log _{10}$-transformation gave

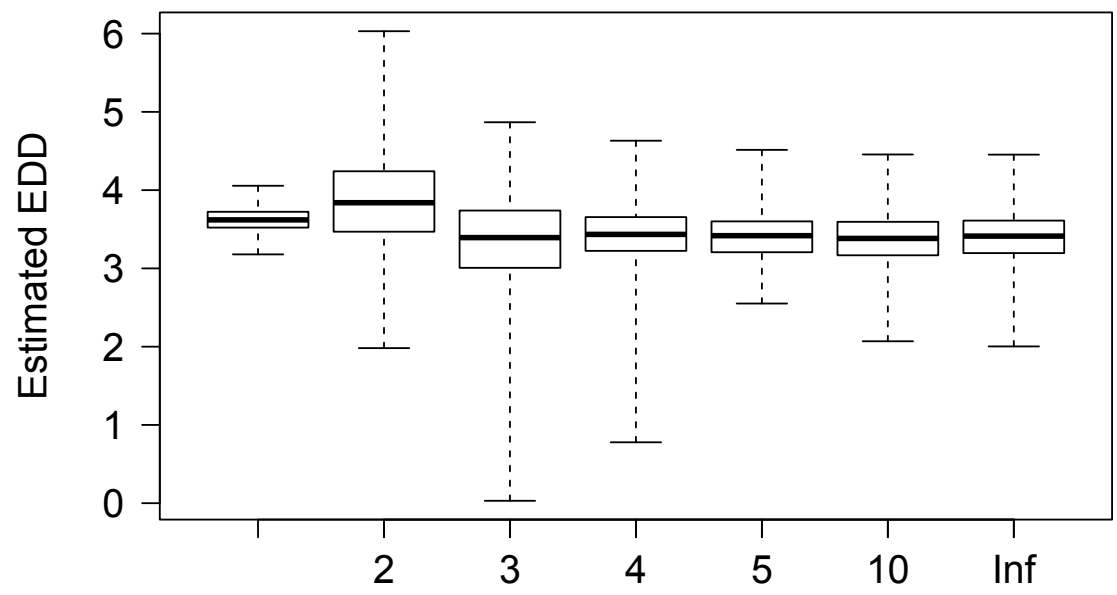

Reference

\section{Bins across mid line}

Figure 3.2. Bootstrapped distributions of estimated effective detection distances $(\mathrm{m})$ using point detection function models applied to different forms of data. Reference indicates un-binned direct distances to first detection position. Mid line models were fitted to distances at which the same records were projected to cross camera's line of sight, with data binned to varying degrees ('Inf' indicates un-binned analysis). Central bars are medians, boxes are interquartile ranges, whiskers are ranges. 


\section{6 | Chapter 3}

Table 3.2. Effective Detection Distance (EDD) of terrestrial mammals in two forest types in the Netherlands, estimated with the marker transect method using single species, single habitat point detection models. Only species with more than twenty distance measurements per habitat type were included.

\begin{tabular}{|c|c|c|c|c|c|c|}
\hline \multirow{3}{*}{ Species } & \multicolumn{3}{|c|}{ Dense understory } & \multicolumn{3}{|c|}{ Open understory } \\
\hline & \multicolumn{2}{|c|}{$\mathrm{AIC}^{\mathrm{a}}$} & \multirow{2}{*}{ EDD $m$ (SE) } & \multicolumn{2}{|c|}{$\mathrm{AIC}^{\mathrm{a}}$} & \multirow{2}{*}{ EDD $m$ (SE) } \\
\hline & Half-normal & Hazard-rate & & Half-normal & Hazard-rate & \\
\hline Pine marten & 44.1 & 46.1 & $2.73(0.30)$ & 74.5 & 76.6 & $3.86(0.35)$ \\
\hline Cat & - & - & - & 57.5 & 60.2 & $4.81(0.63)$ \\
\hline Hare & - & - & - & 391.0 & 400.9 & $4.77(0.21)$ \\
\hline Fox & 94.7 & 91.8 & 3.45 (1.13) & 245.7 & 251.5 & $4.37(0.25)$ \\
\hline Badger & - & - & - & 148.1 & 146.2 & $7.38(0.51)$ \\
\hline Roe deer & 250.6 & 252.4 & $5.95(0.35)$ & 1640.2 & 1654.7 & $6.11(0.15)$ \\
\hline Fallow deer & 349.3 & 348.0 & $7.24(0.77)$ & - & - & - \\
\hline Wild boar & 1616.8 & 1649.9 & $5.40(0.11)$ & - & - & - \\
\hline Red deer & 1327.5 & 1332.7 & $8.45(0.27)$ & - & - & - \\
\hline
\end{tabular}

${ }^{a}$ EDD estimates are given for the best performing model, highlighted in grey.

- represents combinations of species and habitat type that were not observed.

the best fit (Figure 3.3) both in a dense understory (Least Squares regression: $F_{1,4}=23.6, p=0.008, R^{2}$ adj $=0.82, \beta=2.4$ [95\%Cl:1.0-3.8]) and an open understory $\left(F_{1,4}=8.2, p=0.045, R^{2}{ }_{\text {adj }}=0.59, \beta=2.4[95 \% \mathrm{Cl}: 0.1-4.7]\right)$.

We then fitted detection probability functions using multiple-covariate distancesampling models with $\log _{10}$-transformed body mass and habitat as covariates, now using all distance estimates for all species in both habitats. A half-normal model including both covariates had the best fit ( $\mathrm{AIC}=6439.2 ; \beta_{\text {body mass }}=0.40$; $\left.\beta_{\text {habitat }}=0.19\right)$, compared to a half-normal model including only body mass as covariate $(\triangle \mathrm{AIC}=30.6)$ or a hazard-rate model including both covariates $(\triangle \mathrm{AIC}=$ 91.2). Estimates of EDD from the half-normal covariate model are given in Table 3.3.

\section{Discussion}

Variation in the distance over which passing animals are detected is a major source of error in studies that use camera traps for estimating animal abundance ${ }^{54}$. This can be solved by quantifying effective detection distance (EDD). We show that credible estimates of EDD can be obtained from photographs by establishing a line of distance markers in the field of view (FOV) of the camera. This method is a great simplification of previously published methods ${ }^{54,171}$, and the deployment 
of markers in front of all cameras did not substantially increase the time spent in the field, or the costs involved in our survey compared to conventional use of camera traps without the estimation of EDD. Our simulations showed that estimates of EDD acquired with this method resemble estimates using the method proposed by Rowcliffe et al. ${ }^{54}$ if at least 5 intervals were used. We obtained credible EDD estimates for thirteen different mammal species in two different habitats; estimated EDD increased with species body mass and - to a lesser degree - vegetation openness.

We found that the EDD increased with body mass (Figure 3.3), consistent with expectations based on greater emission of heat by larger animals ${ }^{169}$. Similar relationships were found for mammals in Peru ${ }^{180}$ and in Panama ${ }^{54}$. Scaling of EDD with body mass shows that uncorrected photographic capture rates yield overestimates of relative abundance for large species for which sampling effort is effectively larger. This scaling seems to be corresponding to a $\log _{10^{-}}$

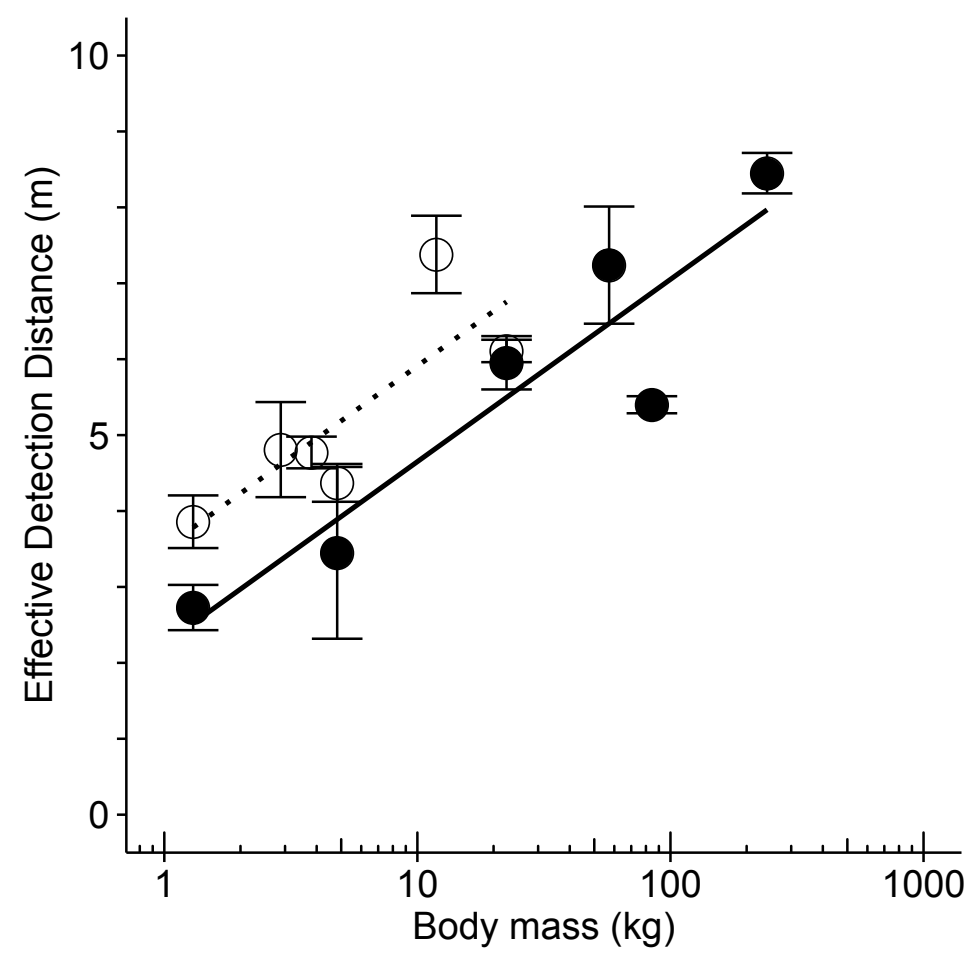

Figure 3.3. Relationship between the Effective Detection Distance (EDD) estimated with single species, single habitat detection functions based on distance intervals obtained from camera trap images using the marker method and body mass of the species. Lines represent linear regression fits for forests with dense understory (filled symbols, solid line) and with open understory (open symbols, dotted line). Whiskers are standard error estimates. 


\section{8 | Chapter 3}

transformation of body mass, which is also consistent with previous findings ${ }^{180,181}$. We used estimates of body mass from the global PanTHERIA database ${ }^{179}$, which might differ from the actual body mass of the local populations, thus introducing error. Therefore, we advise to use body mass estimates obtained from local populations. If age or sex can be distinguished from the camera trap footage, using age- and sex-specific estimates of body mass can further improve precision.

Many camera-trapping studies do not correct for differences between habitats, implicitly assuming that sampling efficiency of camera traps is constant across habitats (e.g., Rovero and Marshall ${ }^{182}$ and Manzo et al. ${ }^{183}$ ). Our field test demonstrates that differences between habitats can be large - $20 \%$ decrease in closed compared to open vegetation in our analysis - and that not accounting for these differences produces biases. For example, apparent differences in habitat use might simply result from vegetation-related differences in detection distance. Avoiding or pruning of vegetation in the FOV may reduce the difference in detectability between sites to some degree, but not entirely. Differences in detection distance in different habitats can be especially problematic when capture rates from camera traps are used to study habitat selection or other variables which are linked to the habitat ${ }^{167}$. Estimating EDD per habitat type and correcting capture rates accordingly can reduce bias in habitat selection studies that use camera trap data.

Our method for estimating EDD relies on the assumption that the distance at which animals cross the midline correlates with the distance at which animals trigger the camera. Our simulation showed that this assumption holds if animals move in a random direction compared to the line of sight of the camera. However, it might no longer hold if animals for some reason tend to always approach from one particular angle. This problem can be overcome by averaging measurements over multiple camera locations. The assumption of random movement of animals compared to the camera position is essential for estimating relative abundance ${ }^{167}$ as well as for estimating density using the random encounter model (REM) ${ }^{170,184}$. Studies using these methods rely on camera placement in random positions, which ensures random movement of animals relative to the camera position. Therefore, our assumptions should hold for camera traps that are deployed for these study purposes, and our method of estimating EDD should give reasonable estimates accordingly.

We found that a minimum of five distance intervals was sufficient for obtaining reliable estimates of EDD (Figure 3.2), but this was based on a simulation with 
Table 3.3. Effective Detection Distance (EDD) of terrestrial mammals in camera-trap surveys in two forest types in the Netherlands estimated with the marker transect method using multiple-covariate point models with $\log _{10}$ body mass and habitat as covariates, using all measurements from all species.

\begin{tabular}{|c|c|c|}
\hline \multirow{2}{*}{ Species $^{a}$} & Dense understory & Open understory \\
\hline & EDD m (SE) & EDD m (SE) \\
\hline Squirrel & $2.40(0.17)$ & $2.90(0.14)$ \\
\hline Hedgehog & $2.79(0.15)$ & $3.37(0.12)$ \\
\hline Polecat & $2.90(0.14)$ & $3.50(0.12)$ \\
\hline Pine marten & $3.05(0.14)$ & $3.68(0.11)$ \\
\hline Stone marten & $3.19(0.13)$ & $3.85(0.11)$ \\
\hline Cat & $3.51(0.12)$ & $4.24(0.10)$ \\
\hline Hare & $3.69(0.11)$ & $4.45(0.09)$ \\
\hline Fox & $3.84(0.11)$ & $4.63(0.09)$ \\
\hline Badger & $4.50(0.09)$ & $5.43(0.08)$ \\
\hline Roe deer & $5.03(0.08)$ & $6.07(0.07)$ \\
\hline Fallow deer & $5.92(0.07)$ & $7.11(0.06)$ \\
\hline Wild boar & $6.34(0.07)$ & $7.58(0.06)$ \\
\hline Red deer & $7.55(0.06)$ & $8.88(0.05)$ \\
\hline
\end{tabular}

a Species are ordered by body mass. Species for which a single species, single habitat estimate of EDD is available in table 3.2 are printed in bold.

a simple detection probability without expansion terms. In reality, detection probability might follow a more complex distribution. For example, detectability at distance zero may be less than $100 \%$ when camera trigger speed is low and animal movement speed is fast, resulting in short-distance detections without photographs of animals and, thus, species-specific distance measurements. In these situations, more precise measurements are needed to model the detection probability. Five distance intervals should thus be considered as a minimum, more is advisable.

Our approach for estimating EDD can be used in the REM for estimating absolute population density ${ }^{54,170}$. So far, most studies using the REM used a proxy to estimate EDD, for example by moving in front of the camera themselves ${ }^{185}$, or by using a domesticated animal, such as a cat ${ }^{183}$. However, our results show that EDD scales with body mass. Thus, the densities found by Manzo et al. ${ }^{183}$ are most probably underestimates, as they used EDD estimated for a domestic cat to estimate pine marten densities, while EDD for pine marten is smaller (Table 3.2).

While most animals ignored the marking sticks, like the Red fox in Figure 3.1, some sniffed at or chewed on the sticks (especially ungulates and carnivores). Because such responses were uncommon, we consider the image sequences obtained using our method suitable for measuring activity and behaviour. 


\section{0 | Chapter 3}

However, the sticks could pose a problem for researchers interested in carnivore or ungulate behaviour. A possible way to overcome behavioural problems could be to take photographs that include markers during the set-up or removal of the camera trap, but not leave any markers during the actual deployment ${ }^{171}$. These photographs can then be used as a reference to overlay all other photographs to measure the distance category at which each animal passes. This might, however, decrease the precision of detection distances as it can be difficult to estimate the right distance interval without physical reference points in front of or behind animals.

Our method allows researchers to correct for differences in capture rate related to variation in EDD, but not for biases caused by differences in the width of the FOV between studies related to the model of camera trap used, openness of the vegetation, or size of the species ${ }^{54}$. This problem can be overcome by considering only animals that cross the midline of the FOV for calculating capture rates, and discarding all observations of animals that do not cross the line. In essence, the sampling is then reduced to a line. A major advantage of this approach is that the capture rates obtain from this line only need to be corrected for EDD and day range (the distance that animals travel daily) to estimate density ${ }^{186}$, just as in line-transect estimation from indirect sign using the Formozov-MalyshevPereleshin formula ${ }^{187}$. Note that this is equivalent to an REM with a detection angle of zero ${ }^{170}$.

In conclusion, our method could facilitate the use of camera traps for estimating relative abundance or density of animal species of which individuals cannot be identified, reducing the bias in relative abundance or density estimates that is due to differences in sampling effort between species and habitats. Our method can be applied by scientists and conservationists all over the world with limited extra effort. We advise to always deploy camera traps with markers - at least during the setup - as to create the possibility to correct capture rates. 


\section{Appendix to Chapter 3}

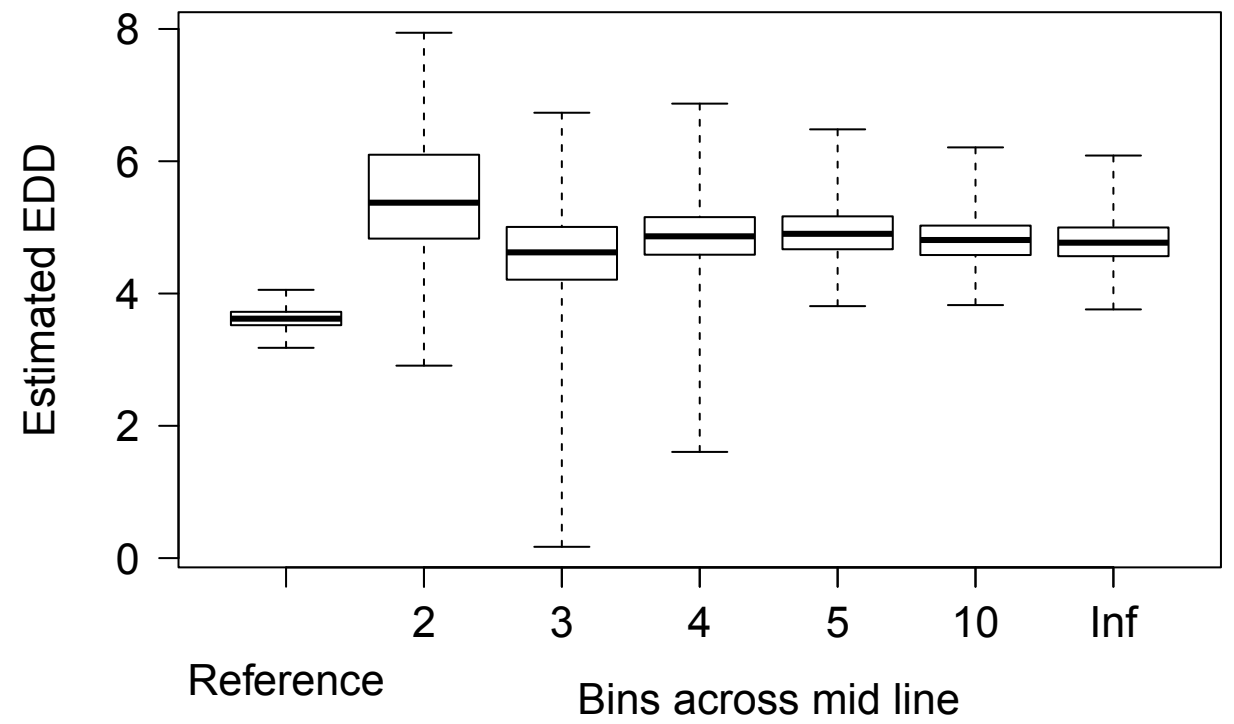

Figure S3.1. Bootstrapped distributions of estimated effective detection distances $(\mathrm{m})$ using line detection function models applied to different forms of data. Reference indicates un-binned direct distances to first detection position. Mid line models were fitted to distances at which the same records were projected to cross camera's line of sight, with data binned to varying degrees ('Inf' indicates un-binned analysis). Central bars are medians, boxes are interquartile ranges, whiskers are ranges.

Table S3.1. Habitat type, year of measurement, location and number of camera deployments of the eight field sites.

\begin{tabular}{|c|c|c|c|}
\hline Site & Year & Coordinates $^{a}$ & $\begin{array}{l}\text { No. of camera } \\
\text { deployments }\end{array}$ \\
\hline \multicolumn{4}{|c|}{ Scots pine with blueberry } \\
\hline Deelerwoud & 2014 & $52^{\circ} 05^{\prime} 51^{\prime \prime} 5^{\circ} 56^{\prime} 42^{\prime \prime} \mathrm{E}$ & 18 \\
\hline Enkhout & 2013 & $52^{\circ} 16^{\prime} 25^{\prime \prime} \mathrm{N} 5^{\circ} 54^{\prime} 49^{\prime \prime} \mathrm{E}$ & 18 \\
\hline Planken Wambuis & 2013 & $52^{\circ} 01^{\prime} 54^{\prime \prime} \mathrm{N} 5^{\circ} 48$ '36”'E & 16 \\
\hline Valenberg & 2014 & $52^{\circ} 15^{\prime} 33^{\prime \prime} \mathrm{N} 5^{\circ} 48^{\prime} 47^{\prime \prime} \mathrm{E}$ & 14 \\
\hline \multicolumn{4}{|c|}{ Pedunculate oak with ferns } \\
\hline \multicolumn{4}{|l|}{ Open understory } \\
\hline Kremboong & 2013 & $52^{\circ} 45^{\prime} 13^{\prime \prime} \mathrm{N} 6^{\circ} 31^{\prime} 16^{\prime \prime} \mathrm{E}$ & 18 \\
\hline Rheebruggen & 2014 & $52^{\circ} 46^{\prime} 60^{\prime \prime} \mathrm{N} \mathrm{6} 6^{\circ} 17^{\prime} 44^{\prime \prime} \mathrm{E}$ & 18 \\
\hline Vledderhof & 2014 & $52^{\circ} 52^{\prime} 46^{\prime \prime} 6^{\circ} 14$ '25”'E & 18 \\
\hline Zwanemeerbos & 2013 & $53^{\circ} 00^{\prime} 46^{\prime \prime} \mathrm{N} 6^{\circ} 45^{\prime} 19^{\prime \prime} \mathrm{E}$ & 18 \\
\hline
\end{tabular}

${ }^{a}$ Coordinates given are the coordinates as measured with a handheld GPS (Garmin eTrex 20) in the middle of the 1 hectare plot. 
62

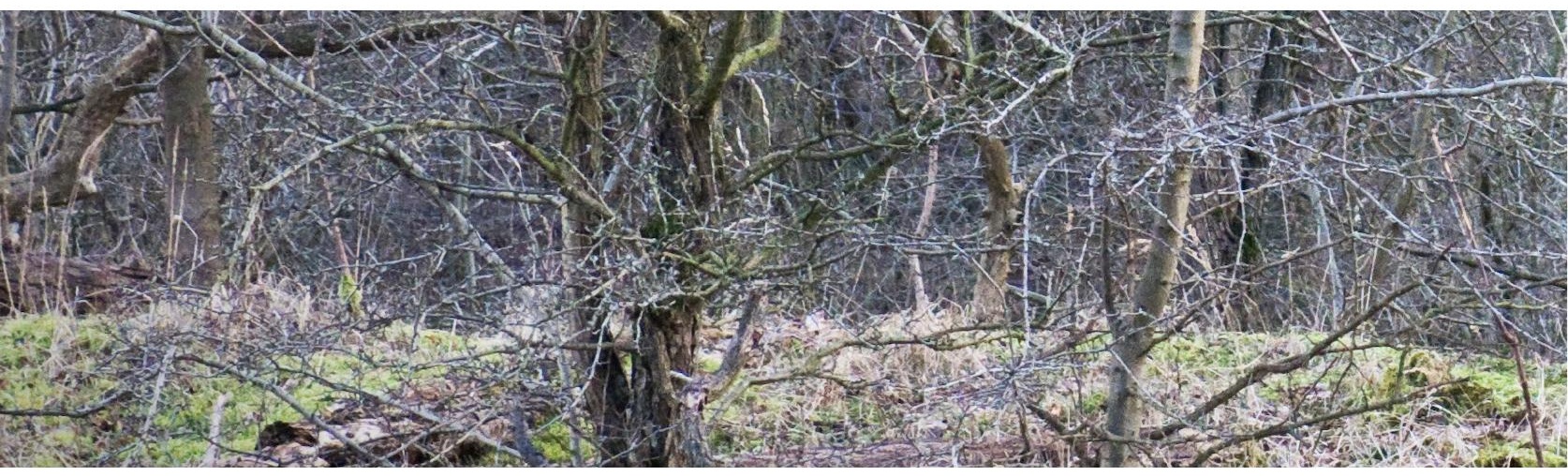

dear, precious deer

merely your presence makes

me tick and alive

Ronald Hofmeester 


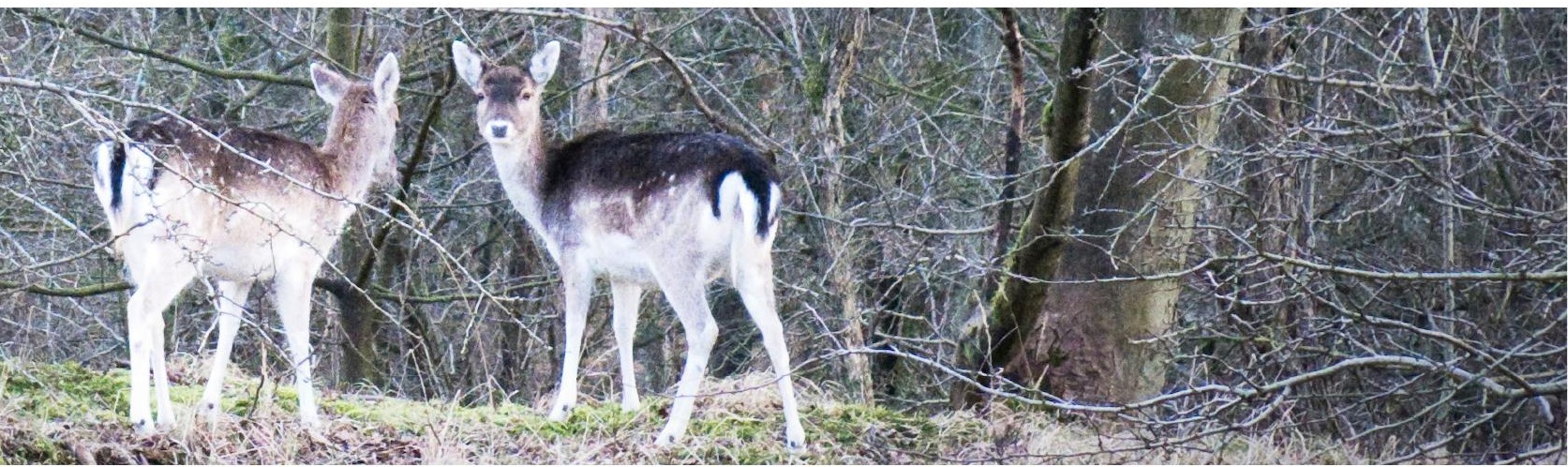

T.R. Hofmeester, H. Sprong, P.A. Jansen, H.H.T. Prins \& S.E. van Wieren

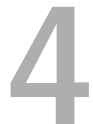

\section{Deer Presence rather than Abundance Deter- mines the Population Density of the Sheep Tick (IXODES RICINUS)}

Understanding which factors drive population densities of disease vectors is an important step in assessing disease risk. We tested the hypothesis that the density of ticks from the Ixodes ricinus complex, which are important vectors for tick-borne diseases, is determined by the density of deer, because adults of these ticks mainly feed on deer. We performed a cross-sectional survey to study I. ricinus density across twenty forest sites in the Netherlands that ranged widely in the relative abundance of deer, and also performed a deer exclosure experiment in four pairs of < 1-ha forest plots. Ixodes ricinus from all stages were more abundant in sites with deer $(n=17)$ than in sites without deer $(n=3)$. Where deer were present, the density of I. ricinus did not increase with the relative abundance of deer with one exception: where only Roe deer were present, larval density increased with the relative abundance of Roe deer. Experimental exclosure of deer reduced nymph density by $66 \%$ and adult density by $32 \%$ within a timeframe of two years. Our results suggest that deer presence rather than deer abundance drives the density of I. ricinus nymphs and adults. This implies that $I$. ricinus density will not necessarily decrease with a reduction in deer density. That small exclosures ( $<1$ ha) substantially reduced $I$. ricinus densities suggests that fencing can be used to reduce tick-borne disease risk in areas with high recreational pressure. 


\section{4 | Chapter 4}

\section{Introduction}

Ticks are important vectors for diseases such as Lyme borreliosis, Rocky Mountain spotted fever and tick-borne encephalitis ${ }^{57}$. Understanding which factors drive population densities of ticks is an important step in assessing disease risk ${ }^{188}$. Most tick species spent part of their life in the vegetation searching for a host from which they need to acquire blood in order to survive and reproduce ${ }^{189}$. The number of bloodmeal hosts available in the environment will determine the likelihood of a tick finding a host ${ }^{8}$. Therefore, the presence and density of hosts is considered an important determinant of tick density.

Ticks from the Ixodes ricinus complex have three blood feeding life stages larva, nymph and adult ${ }^{36,57,59}$ - all of which search for a host by questing in the vegetation, but do so at different heights, probably related to differences in host preference ${ }^{40}$. Most larvae feed from small mammals, while most adult ticks feed on deer ${ }^{190}$ (Chapter 2). It is widely assumed that deer are essential hosts for ticks in the $I$. ricinus complex. Indeed, some studies found a strong correlation of tick density with deer presence and density ${ }^{38,191,192}$. Several other studies, however, found that deer exclusion (by fencing) and deer culling did not always reduce tick densities ${ }^{193,194}$. Thus, it is still unclear whether and how management of deer populations reduces tick densities.

Three modelling studies that took into account the complex life cycle of ticks with multiple host species suggested that the relationship between deer and tick densities is non-linear, and different for the different stages ${ }^{27,195,196}$. Van Buskirk and Ostfeld ${ }^{27}$, for example, modelled how nymph densities of $I$. scapularis responded to differences in densities of hosts for larvae and adults, and found that the density of hosts for adults was limiting nymph density only at very low host densities, where the availability of hosts for larvae then became limiting. Thus, nymph and adult density appear related to deer density according to a nonlinear threshold relationship, rather than the linear relationship used in most studies (e.g., Gilbert et al. ${ }^{38}$ and Sprong et al. ${ }^{39}$ ). Furthermore, Van Buskirk and Ostfeld ${ }^{27}$ suggested that the threshold adult-host density is close to zero.

Here, we empirically test these predictions for the relationship between $I$. ricinus and three species of deer - Roe deer (Capreolus capreolus), Red deer (Cervus elaphus) and Fallow deer (Dama dama) - in forests in the Netherlands. We used a cross-sectional study across twenty forest plots to test the hypotheses that $I$. ricinus densities of all stages would be lower in areas where deer are 
absent compared to areas where deer are present, and that the relationship between the number of deer and questing $I$. ricinus density is linear for larvae but not for nymphs or adult ticks. Furthermore we compared tick densities between experimental deer exclosures and control plots at four forest sites to test if deer are indeed essential hosts for I. ricinus.

\section{Methods}

Study sites

The cross-sectional study encompassed twenty 1-ha plots in nineteen forested areas in the Netherlands (Table S4.1), which were $>5 \mathrm{~km}$ apart. We sampled eleven plots in 2013 and nine plots in 2014. All plots were positioned within forested areas with Pedunculate oak (Quercus robur), Scots pine (Pinus sylvestris), or a combination of these (mixed forest) as dominant tree species (Table S4.1), and selected based on distribution patterns of deer in the Netherlands ${ }^{197}$. One study area, Enkhout, had two plots just $150 \mathrm{~m}$ apart, but one of these was located in a 3-ha stand fenced three years prior to the study, that thus had no ungulates.

For the experimental deer removal study, we placed four exclosures in a forested area near Apeldoorn, the Netherlands (52 $\left.14^{\prime} \mathrm{N}, 5^{\circ} 55^{\prime} \mathrm{E}\right)$ following a Before - After Control - Impact design. The fences (2.2 m high) were erected in May 2013 and included $0.61-0.78$ ha of mixed forest with an understory dominated by Blueberry (Vaccinium myrtillus). Each exclosure had a control plot with a similar vegetation and forest structure, ca. $100 \mathrm{~m}$ away from the exclosure. These plots were sampled twice, in 2013 and 2015.

\section{Relative abundance of deer}

The likelihood of a tick encountering a deer principally depends on the frequency of deer passage. We measured the average frequency of deer passage between March and November, the main activity season of Ixodes ricinus in the Netherlands ${ }^{156}$ by running camera traps at 18 random points per plot. Two camera traps (HC500, RECONYX Inc., Holmen, WI, U.S.A.) were deployed in nine consecutive rounds of four weeks at sets of two random points $>30 \mathrm{~m}$ apart, as to minimize the chance of one animal walking past both cameras in a short amount of time, resulting in 18 deployments and 504 sampling days (camera malfunction and theft reduced the sample size at some sites; Table S4.1). Cameras were placed on a tree nearest to a computer-generated random point, 


\section{6 | Chapter 4}

at $40 \mathrm{~cm}$ above the ground with the view parallel to the ground, and without bait or lure. We set the camera traps to the highest sensitivity, to take a series of ten pictures when triggered with no delay, and to take time-lapse photos every twelve hours to generate a record of functioning. We used an automated image processing tool ${ }^{178}$ in which sequences were combined per event.

We quantified the effective detection distance (EDD) for each species and habitat type using the line-transect method ${ }^{198}$ (Chapter 3 ) to control for variation in detectability between species. This involved the placement of a line of markers in the centre of the view at distance intervals of $2.5 \mathrm{~m}$. For each animal passage, we recorded the species and distance from the camera at which the animal walked through the markers. Using these data, we estimated the EDD for all mammal species per habitat type using a point model with a detection probability described by a half-normal function with $\log _{10}$-transformed body mass as covariate ${ }^{198}$ (Chapter 3 ). The EDD estimates for the three deer species are given in Table S4.2. These estimates were used to determine passage rates per species per camera location as,

$$
P R_{i}=\frac{x_{i}}{t \cdot E D D_{i_{h}}}
$$

Equation 4.1

where $P R_{i}$ is the passage rate of species $i\left(\right.$ meter $^{-1}$ day $\left.^{-1}\right), x_{i}$ the number of passages of species $i, t$ the total time at which the camera was active (in days), and $E D D_{\text {in }}$ the effective detection distance of species $i$ in habitat type $h$ (in meters). Records of animals that did not cross the line of markers were discarded. By doing so, we eliminated the need to correct for effective detection angle ${ }^{54}$. For each plot, we calculated an average passage rate $\left(\mathrm{m}^{-1} \mathrm{~d}^{-1}\right)$ per species by using the arithmetic mean. The passage rates of all deer species were summed to determine deer passage rate. For clarity we will refer to passage rates as "relative abundance"166, but strictly speaking it is a combination of density and activity in a plot.

For the experimental study, we used camera traps during July - November 2015 to assess the presence or absence of deer in all plots. In each plot, 20 locations were sampled for one week using two cameras per plot with an intercamera spacing of $>30 \mathrm{~m}$. Camera settings and placement were identical to the cross-sectional study and the same custom-made image processing tool was used to process the photographs. 


\section{Questing tick density}

For the cross-sectional study, each site was visited six times, once every four weeks in the period April - September, to collect ticks by blanket-dragging twenty transects of $10 \mathrm{~m}$ using a $1 \mathrm{~m}^{2}$ white cotton cloth ${ }^{199}$. We only dragged for ticks on dry days, with an air temperature $>10^{\circ} \mathrm{C}$ and on dry vegetation ${ }^{8,40}$, and minimized variation in weather conditions between plots by visiting all plots within five days during each session. After each 10-m drag we counted all larval, nymphal and adult $I$. ricinus on the cloth, and used these numbers to determine an average density of questing ticks $\left(100 \mathrm{~m}^{-2}\right)$ for each stage per plot.

For the experimental study, we estimated tick density four times: In May and August 2013, just after the fences were placed, and again in May and August 2015, after two years of treatment. During each visit, fifteen transects of $10 \mathrm{~m}$ were dragged, and all nymph and adults on the cloth counted, to estimate the average density of questing ticks $\left(100 \mathrm{~m}^{-2}\right)$ for each stage for both years. We did not count larvae.

\section{Statistical analysis}

Hypotheses were tested in R 3.2.381, using generalized linear mixed models (GLMM) with a negative binomial distribution and log link function, as implemented in the gImmADMB package ${ }^{200,201}$. For the cross-sectional study, we performed two separate tests. First, we tested for a difference in I. ricinus density per stage between sites with and without deer. Second, we tested for a correlation of $I$. ricinus densities per life stage with deer passage rate, using only the plots with deer presence. To test if Roe deer was more important than Fallow deer and Red deer, we tested for a correlation between I. ricinus density and Roe deer passage rate, including the plots with only Roe deer presence. The intercept was allowed to differ between the two sampling years in all models.

For the experimental study we tested for differences in density of nymphs and adults between control plots and exclosures, and between years including an interaction term, using a random intercept per plot nested within site to correct for repeated measurements within each plot (one measurement in 2013 and one in 2015) and the paired design (one exclosure and one control plot within each site). To test for a significant difference between exclosures and control plots after two years of treatment we performed a GLMM with a poisson distribution and log link function, as these had a better fit (lower AICc value ${ }^{82}$ ) than GLMMs with a negative binomial distribution. 


\section{8 | Chapter 4}

\section{Results}

\section{Cross-sectional study}

Three sites - the Enkhout exclosure, Pettemerduinen and Schoorlse Duinen lacked deer. In the other seventeen sites, deer passage rate ranged from 0.01 - $0.84 \mathrm{~m}^{-1} \mathrm{~d}^{-1}$ (Table S4.3). Roe deer were present in fifteen sites with passage rates ranging six-fold, from $0.01-0.06 \mathrm{~m}^{-1} \mathrm{~d}^{-1}$, Fallow deer were present in just five sites, with passage rates ranging more than 800 -fold, from $0.001-0.84$ $\mathrm{m}^{-1} \mathrm{~d}^{-1}$, and Red deer were present in four sites with passage rates ranging fourfold, from $0.01-0.04 \mathrm{~m}^{-1} \mathrm{~d}^{-1}$ (Table S4.3).

A total of 38,566 larvae, 16,617 nymphs and 1,019 adults of Ixodes ricinus were collected in the twenty sites. Larval density ranged from $0-517100 \mathrm{~m}^{-2}$, nymphal density from $1.8-183.4100 \mathrm{~m}^{-2}$ and adult density from $0.3-13.4$ $100 \mathrm{~m}^{-2}$ (Table S4.3). Larval densities were on average 99.99\% lower (and nearly zero) in plots without deer than in plots with deer (GLMM: $\beta=8.8, p<0.001$; Figure 4.1A). Nymphal densities were on average 93.1\% lower in plots without deer than in plots with deer (GLMM: $\beta=2.7, p<0.001$, Figure $4.1 \mathrm{C}$ ) and adult densities on average $71.4 \%$ lower (GLMM: $\beta=1.3, p=0.007$, Figure $4.1 \mathrm{E}$ ), as expected. In plots with deer, neither larval density (GLMM: $\beta=1.1, p=0.06$, Figure 4.1B), nor nymphal (GLMM: $\beta=0.05, p=0.91$, Figure 4.1D) or adult density (GLMM: $\beta=0.5, p=0.38$ Figure $4.1 F$ ) was correlated with the relative abundance of deer. In the 10 plots with only Roe deer present, larval densities increased with Roe deer passage rate (GLMM: $\beta=3.2, p=0.05)$, but nymphal density (GLMM: $\beta=1.2, p=0.22$ ) and adult density (GLMM: $\beta=2.0, p=0.10$ ) did not.

\section{Experimental study}

Camera traps detected Roe deer and Red deer in all control plots in 2015, but photographed none in any of the exclosures, confirming that the exclosures were effective in excluding deer. We collected a total of 1,691 nymphs and 82 adults of $I$. ricinus in 2013 and 429 nymphs and 32 adults of $I$. ricinus in 2015. There was no initial difference in nymphal densities between exclosures and controls (GLMM: difference $=0.1, p=0.57$; Figure 4.2A). Two years later, nymphal densities were significantly lower in all plots (GLMM: difference $=-1.1, p<0.001$; Figure $4.2 A)$, and nymphal densities were significantly lower (66\%) in exclosures than in control plots (GLMM: difference $=-0.8, p<0.001$; Figure 4.2A). Exclosures 

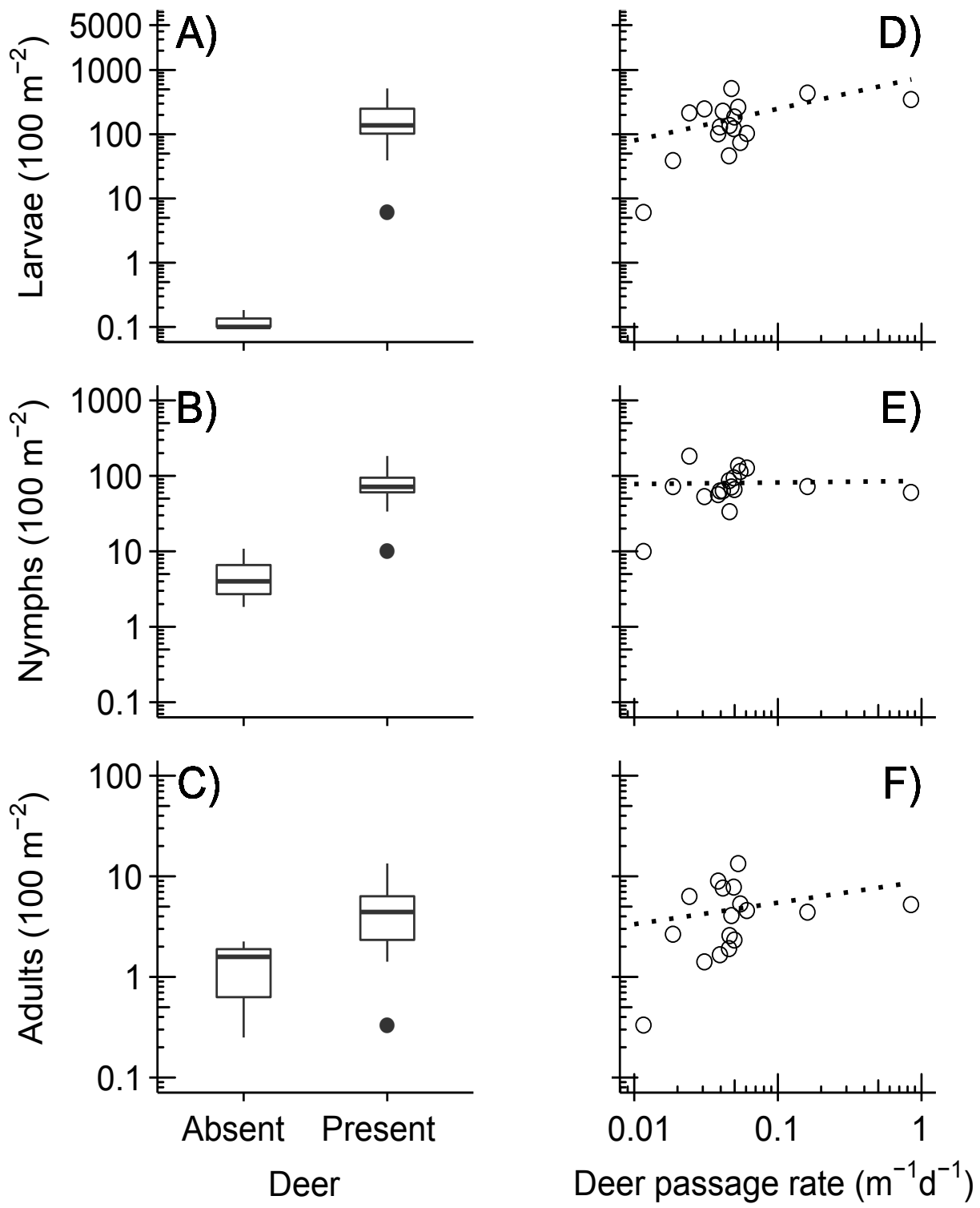

Figure 4.1. The density of the Sheep tick Ixodes ricinus differed significantly $(p<0.01)$ between 17 forest plots with deer and 3 plots without (A-C), but did not significantly increase with the relative abundance of deer (D-F), for larvae $(A, D$; density +0.1$)$, nymphs $(B, E)$ and adults $(C, F)$. Dotted lines represent generalized linear mixed model fit for non-significant models. 


\section{0 | Chapter 4}

had higher initial adult densities than controls (GLMM: difference $=0.7, p=$ 0.04; Figure 4.2B). Two years later, adult densities had significantly reduced in exclosures (GLMM: difference $=-0.7, p<0.001$; Figure 4.2B), but not in control plots (GLMM: difference $=-0.35, p=0.41$; Figure $4.2 B$ ), resulting in lower $(32 \%)$ adult density in exclosures than in control plots (Figure 4.2B).

\section{Discussion}

Deer density is widely considered the most important determinant of the density of ticks in the Ixodes ricinus complex (e.g., Gray ${ }^{36}$ ), but the shape of this relationship remains poorly known. We studied the relationship between the relative abundance of deer (deer passage rate) and tick densities in a cross-sectional study of twenty forested plots, and performed a deer exclosure experiment. We found that $I$. ricinus density was on average $71-99 \%$ lower in plots without deer than in plots with deer, depending on the life stage (Figure 4.1). We found no additional correlation of $I$. ricinus density with the relative abundance of deer for any of the life stages (Figure 4.1), with one exception: in plots with Roe deer only, larval density increased with the relative abundance of Roe deer. Excluding deer from small ( $<1 \mathrm{ha}$ ) forest plots using a fence decreased nymphal and adult $I$. ricinus densities with $66 \%$ and $32 \%$, respectively (Figure 4.2). We therefore conclude that $I$. ricinus requires deer, but that just few deer are needed. Furthermore, we found that fencing reduces I. ricinus populations even at small spatial scales.

We found a significantly lower number of questing ticks of all three stages in sites where deer were absent compared to sites with deer presence (Figure 4.1). This result is in agreement with two earlier studies on ticks from the I. ricinus complex that found a dramatic decrease in tick densities after elimination of deer populations ${ }^{192,202}$. In our two sites where deer were naturally absent, alternative hosts for adult ticks were available in the form of European hare (Lepus europaeus), European hedgehog (Erinaceus europaeus), European pine marten (Martes martes), or Red fox (Vulpes vulpes), as indicated by our camera trap data. Although all of these host species can support feeding adult I. ricinus ${ }^{190}$ (Chapter 2), their presence appeared insufficient for sustaining an I. ricinus population, as questing larvae were nearly absent where there were no deer. The difference was smaller, but still substantial, for the nymphal and adult stages. This can be explained by immigration of larvae and nymphs 

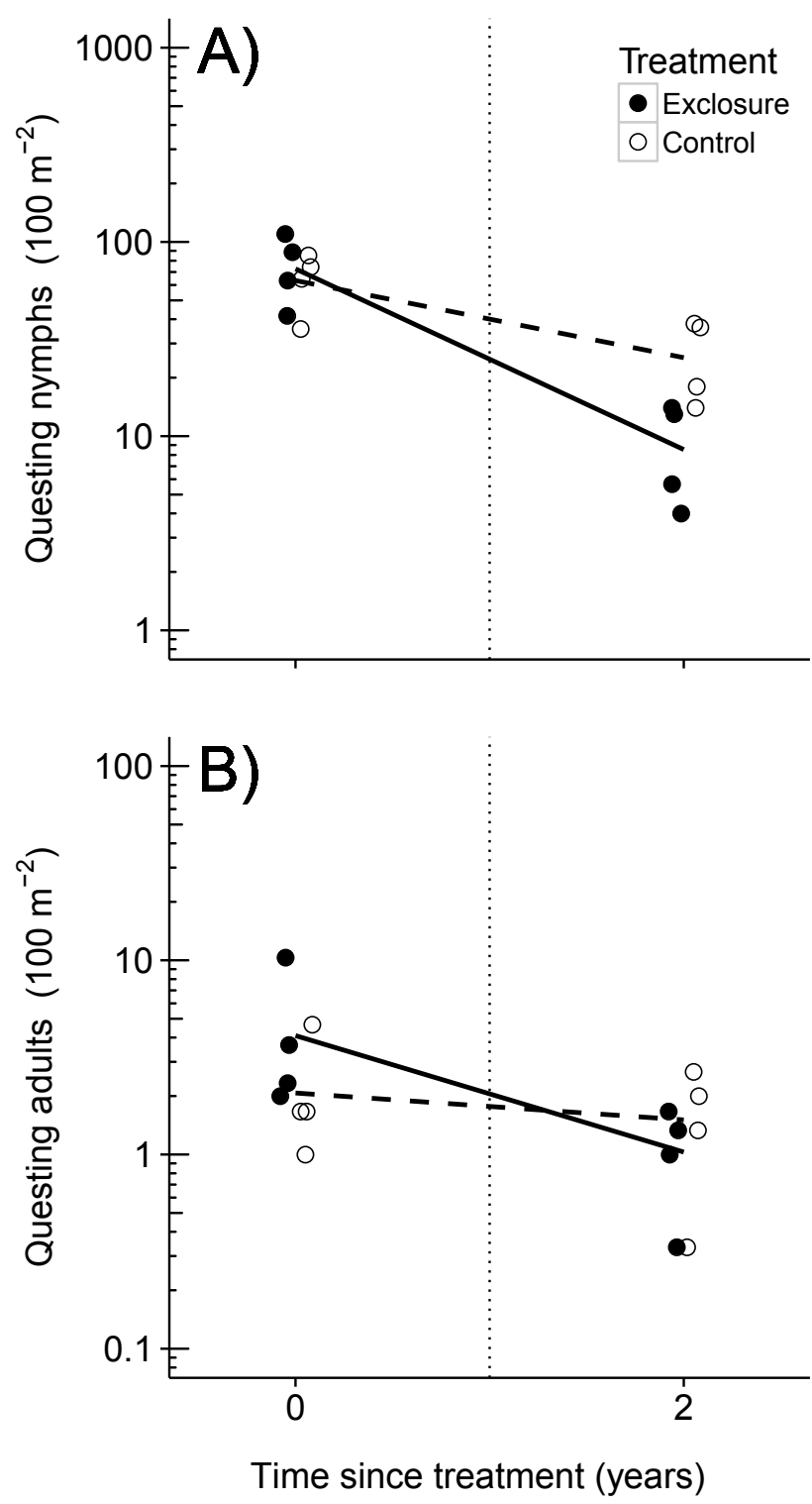

Figure 4.2. Deer exclusion experiment showing that excluding deer depressed the density of questing I. ricinus nymphs A) and adults B) over two years. Lines connect the average tick densities for both years in exclosures (solid lines) and controls (dashed lines) to illustrate the differences between the treatments. 


\section{2 | Chapter 4}

with a host ${ }^{10}$. Therefore, our results imply that complete elimination of deer substantially reduces questing $I$. ricinus densities, even in the presence of alternative hosts for the adult stage.

We found that exclusion of deer from forest plots that measured $<1$ ha caused a substantial reduction of $I$. ricinus, as much as $66 \%$ for nymphs. Our findings agree with Gilbert et al. ${ }^{38}$, who found a strong decrease in the density of $I$. ricinus nymphs in both large and small ( $<1$ ha) exclosures. However, they disagree with a meta-analysis by Perkins et al. ${ }^{194}$ who found that exclosures $>2.5$ ha were needed to reduce densities of I. scapularis and Amblyomma americanum. Perkins et al. ${ }^{194}$ also studied the number of $I$. ricinus parasitizing on small rodents in two small ( $<1$ ha) forest plots in Italy, and found no difference in larval burden and an increase in nymphal burden on small rodents in exclosures compared to control plots ${ }^{194}$, again suggesting that small exclosures do not effectively reduce I. ricinus densities. The discrepancy may be caused by the fact that Perkins et al. ${ }^{194}$ included studies in which effects of exclosures on tick densities were measured just 1 year after placement, while a reduction in the number of questing nymphs would only be expected after two years, given the multi-year life cycle of ticks in the I. ricinus complex. Failure of adult ticks to find deer as host should result in a collapse of the number of larvae after one year, and collapse of the number of nymphs after two years ${ }^{203}$. The number of questing ticks can even increase initially after exclosure placement, as a result of the reduced chance of finding a host, as we observed in 2013 for adult $I$. ricinus (Figure 4.2). Therefore, we conclude that small exclosures can in fact reduce densities of questing $I$. ricinus.

Van Buskirk and Ostfeld ${ }^{27}$ predicted that the relationship between deer density and questing nymphal Ixodes density follows a threshold relationship. Our results support this prediction, as the density of none of the I. ricinus life stages increased with the relative abundance of deer. Only in areas with only Roe deer did we find a correlation between the relative abundance of Roe deer and larval $I$. ricinus density. These results agree with a study on I. scapularis in which large variation in deer density did not result in changes in questing nymphal density, while there was a weak correlation with larval density ${ }^{204}$. Such a threshold relationship was already suggested by Wilson et al. ${ }^{202}$ and can explain equivocal effects of deer culling on densities of ticks in the I. ricinus complex ${ }^{193}$. The correlation between the relative abundance of Roe deer and I. ricinus larval density in combination with a lack of correlation for the other 
stages suggests that as soon as deer are present, the hosts for larvae become limiting in determining I. ricinus nymphal densities.

Our findings are in contrast to previous studies that showed a linear relationship ${ }^{38,39}$ or a parabolic relationship ${ }^{205}$ between deer density and $I$. ricinus density. This difference could be due to differences in host assemblage composition between different studies. Small mammals are considered the most important hosts for larval I. ricinus ${ }^{190}$ (Chapter 2), however, when small mammals are absent, deer are able to feed a substantial number of larvae. This might be the explanation for a linear correlation of $I$. ricinus density and estimates of deer density in several studies in Scotland ${ }^{38,206-208}$ where small mammals occur in low abundance and carry few ticks in some habitats ${ }^{209}$. Therefore, deer are important hosts for I. ricinus, but the other hosts in the vertebrate assemblage need to be considered as well when studying relationships between host abundance and $I$. ricinus density.

Our findings have implications for the possibility of deer management for the control of $I$. ricinus densities. We argue that reduction of deer densities may not reduce the density of $I$. ricinus nymphs in areas where deer are not the principle host for larvae, unless deer are eliminated. Moreover, low deer densities may enhance dispersal of deer from other areas, which will most probably counteract any effects of deer management on $I$. ricinus density ${ }^{196}$. Thus, deer culling might not be an effective strategy to reduce I. ricinus densities. In contrast, excluding deer by fencing the area can be used to decrease I. ricinus densities, even at small spatial scales. This effect could for example be used to reduce the risk of acquiring a tick bite in areas with high recreational pressure, such as campsites and playgrounds. 


\section{4 | Chapter 4}

\section{Appendix to Chapter 4}

Table S4.1. Characteristics and sampling effort (camera days) of the research sites.

\begin{tabular}{|c|c|c|c|c|c|}
\hline Site & Habitat & $\begin{array}{l}\text { Undergrowth } \\
\text { vegetation }^{\mathrm{a}}\end{array}$ & Year & Coordinates $^{b}$ & $\begin{array}{l}\text { No. camera } \\
\text { trapping days }\end{array}$ \\
\hline $\begin{array}{l}\text { Amsterdamse Waterleiding } \\
\text { Duinen }\end{array}$ & Mixed forest & $\begin{array}{l}\text { Calamagrostis } \\
\text { epigejos }\end{array}$ & 2014 & $\begin{array}{l}52^{\circ} 20^{\prime} 36^{\prime \prime} \mathrm{N} \\
4^{\circ} 33^{\prime} 58^{\prime \prime} \mathrm{E}\end{array}$ & 492 \\
\hline Bergherbos & Mixed forest & $\begin{array}{l}\text { Deschampsia } \\
\text { flexuosa }\end{array}$ & 2013 & $\begin{array}{l}51^{\circ} 55^{\prime} 14^{\prime \prime} \mathrm{N} \\
6^{\circ} 14{ }^{\prime} 30^{\prime \prime} \mathrm{E}\end{array}$ & 504 \\
\hline Buunderkamp & $\begin{array}{l}\text { Scots pine } \\
\text { forest }\end{array}$ & $\begin{array}{l}\text { Vaccinium } \\
\text { myrtillus }\end{array}$ & 2013 & $\begin{array}{l}52^{\circ} 00^{\prime} 56^{\prime \prime} \mathrm{N} \\
5^{\circ} 44^{\prime} 50^{\prime \prime} \mathrm{E}\end{array}$ & 504 \\
\hline Duin en Kruidberg & Mixed forest & $\begin{array}{l}\text { Calamagrostis } \\
\text { epigejos }\end{array}$ & 2013 & $\begin{array}{l}52^{\circ} 26^{\prime} 16^{\prime \prime} \mathrm{N} \\
4^{\circ} 36^{\prime} 18^{\prime \prime} \mathrm{E}\end{array}$ & 504 \\
\hline Deelerwoud & $\begin{array}{l}\text { Scots pine } \\
\text { forest }\end{array}$ & $\begin{array}{l}\text { Vaccinium } \\
\text { myrtillus }\end{array}$ & 2014 & $\begin{array}{l}52^{\circ} 05^{\prime} 51^{\prime \prime} \mathrm{N} \\
5^{\circ} 56^{\prime} 42^{\prime \prime} \mathrm{E}\end{array}$ & 504 \\
\hline Enkhout & $\begin{array}{l}\text { Scots pine } \\
\text { forest }\end{array}$ & $\begin{array}{l}\text { Vaccinium } \\
\text { myrtillus }\end{array}$ & 2013 & $\begin{array}{l}52^{\circ} 16^{\prime} 25^{\prime \prime} \mathrm{N} \\
5^{\circ} 54^{\prime} 49^{\prime \prime} \mathrm{E}\end{array}$ & $495 / 504^{c}$ \\
\hline Herperduin & Mixed forest & Molinia caerulea & 2014 & $\begin{array}{l}51^{\circ} 45^{\prime} 33^{\prime \prime} N \\
5^{\circ} 36^{\prime} 53^{\prime \prime} \mathrm{E}\end{array}$ & 504 \\
\hline Halfmijl & Mixed forest & Molinia caerulea & 2013 & $\begin{array}{l}51^{\circ} 25^{\prime} 23^{\prime \prime} \mathrm{N} \\
5^{\circ} 19^{\prime} 09^{\prime \prime} \mathrm{E}\end{array}$ & 504 \\
\hline Kremboong & $\begin{array}{l}\text { Pedunculate } \\
\text { oak forest }\end{array}$ & $\begin{array}{l}\text { Dryopteris } \\
\text { dilatata }\end{array}$ & 2013 & $\begin{array}{l}52^{\circ} 45^{\prime} 13^{\prime \prime} \mathrm{N} \\
6^{\circ} 31^{\prime} 16^{\prime \prime} \mathrm{E}\end{array}$ & 504 \\
\hline Maashorst & Mixed forest & $\begin{array}{l}\text { Deschampsia } \\
\text { flexuosa }\end{array}$ & 2014 & $\begin{array}{l}51^{\circ} 42^{\prime} 44^{\prime \prime} N \\
5^{\circ} 35^{\prime} 24^{\prime \prime} \mathrm{E}\end{array}$ & 504 \\
\hline Pettemerduin & $\begin{array}{l}\text { Pedunculate } \\
\text { oak forest }\end{array}$ & $\begin{array}{l}\text { Polypodium } \\
\text { vulgare }\end{array}$ & 2014 & $\begin{array}{l}52^{\circ} 46^{\prime} 33^{\prime \prime} \mathrm{N} \\
4^{\circ} 40^{\prime} 19^{\prime \prime} \mathrm{E}\end{array}$ & 499 \\
\hline Planken Wambuis & $\begin{array}{l}\text { Scots pine } \\
\text { forest }\end{array}$ & $\begin{array}{l}\text { Vaccinium } \\
\text { myrtillus }\end{array}$ & 2013 & $\begin{array}{l}52^{\circ} 01^{\prime} 544^{\prime \prime} \mathrm{N} \\
5^{\circ} 48^{\prime} 36^{\prime \prime} \mathrm{E}\end{array}$ & 441 \\
\hline Rheebruggen & $\begin{array}{l}\text { Pedunculate } \\
\text { oak forest }\end{array}$ & $\begin{array}{l}\text { Dryopteris } \\
\text { dilatata }\end{array}$ & 2014 & $\begin{array}{l}52^{\circ} 46^{\prime} 60^{\prime \prime} \mathrm{N} \\
6^{\circ} 17^{\prime} 44^{\prime \prime} \mathrm{E}\end{array}$ & 504 \\
\hline Schoorlse Duinen & Mixed forest & Molinia caerulea & 2013 & $\begin{array}{l}52^{\circ} 41^{\prime} 47^{\prime \prime} \mathrm{N} \\
4^{\circ} 40^{\prime} 01^{\prime \prime} \mathrm{E}\end{array}$ & 504 \\
\hline Stameren & Mixed forest & $\begin{array}{l}\text { Deschampsia } \\
\text { flexuosa }\end{array}$ & 2014 & $\begin{array}{l}52^{\circ} 03^{\prime} 38^{\prime \prime} \mathrm{N} \\
5^{\circ} 21^{\prime} 01^{\prime \prime} \mathrm{E}\end{array}$ & 486 \\
\hline Valenberg & $\begin{array}{l}\text { Scots pine } \\
\text { forest }\end{array}$ & $\begin{array}{l}\text { Vaccinium } \\
\text { myrtillus }\end{array}$ & 2014 & $\begin{array}{l}52^{\circ} 15^{\prime} 33^{\prime \prime} \mathrm{N} \\
5^{\circ} 48^{\prime} 47^{\prime \prime} \mathrm{E}\end{array}$ & 391 \\
\hline
\end{tabular}


Table S4.1. Continued

\begin{tabular}{|c|c|c|c|c|c|}
\hline Site & Habitat & $\begin{array}{l}\text { Undergrowth } \\
\text { vegetation }^{a}\end{array}$ & Year & Coordinates $^{b}$ & $\begin{array}{l}\text { No. camera } \\
\text { trapping days }\end{array}$ \\
\hline Vijverhof & Mixed forest & $\begin{array}{l}\text { Deschampsia } \\
\text { flexuosa }\end{array}$ & 2013 & $\begin{array}{l}52^{\circ} 09^{\prime} 43^{\prime \prime} \mathrm{N} \\
5^{\circ} 13^{\prime} 43^{\prime \prime} \mathrm{E}\end{array}$ & 507 \\
\hline Vledderhof & $\begin{array}{l}\text { Pedunculate } \\
\text { oak forest }\end{array}$ & $\begin{array}{l}\text { Dryopteris } \\
\text { dilatata }\end{array}$ & 2014 & $\begin{array}{l}52^{\circ} 52^{\prime} 46^{\prime \prime} \mathrm{N} \\
6^{\circ} 14^{\prime} 25^{\prime \prime} \mathrm{E}\end{array}$ & 504 \\
\hline Zwanemeerbos & $\begin{array}{l}\text { Pedunculate } \\
\text { oak forest }\end{array}$ & $\begin{array}{l}\text { Pteridium aqui- } \\
\text { linum }\end{array}$ & 2013 & $\begin{array}{l}53^{\circ} 00^{\prime} 46^{\prime \prime} \mathrm{N} \\
6^{\circ} 45^{\prime} 19^{\prime \prime} \mathrm{E}\end{array}$ & 504 \\
\hline
\end{tabular}

a The given plant species was the most dominant species in the herbaceous layer in the 1 hectare plot.

${ }^{\mathrm{b}}$ Coordinates given are the coordinates as measured with a handheld GPS (Garmin eTrex 20) in the middle of the 1 hectare plot.

${ }^{c}$ The number of camera trapping days is given for the plot outside of the exclosure and the plot inside the exclosure, which differed due to a defect camera during one of the deployments in the plot outside the exclosure.

Table S4.2. Estimated Effective Detection Distances (EDD) of the camera traps per habitat type, for the three deer species in this study.

\begin{tabular}{lllllll}
\hline \multirow{2}{*}{ Species } & \multirow{2}{*}{$\begin{array}{l}\text { Body mass } \\
(\mathrm{kg})^{\mathrm{a}}\end{array}$} & \multicolumn{5}{c}{$\mathrm{EDD}^{\mathrm{a}}(\mathrm{m})$ by Habitat $^{\mathrm{b}}$} \\
\cline { 3 - 7 } & & $\mathrm{CE}^{\mathrm{c}}$ & $\mathrm{DF}^{\mathrm{c}}$ & $\mathrm{FE}^{\mathrm{c}}$ & $\mathrm{MC}^{\mathrm{c}}$ & $\mathrm{VM}^{\mathrm{c}}$ \\
\hline Fallow deer (Dama dama) & 57.2 & 6.2 & - & 7.2 & - & 5.9 \\
Red deer (Cervus elaphus) & 240.9 & - & - & - & - & 7.6 \\
Roe deer (Capreolus capreolus) & 22.5 & 5.7 & 6.0 & 6.1 & 6.3 & 5.0 \\
\hline
\end{tabular}

\footnotetext{
a Body mass values as given in the PanTHERIA database ${ }^{179}$

${ }^{\mathrm{b}}$ Dominant plant species in the understorey. $\mathrm{CE}=$ Wood small-reed Calamagrostis epigejos, DF $=$ Wavy hairgrass Deschampsia flexuosa, FE = ferns (Broad buckler-fern Dryopteris dilatata, Common polypody Polypodium vulgare or Bracken Pteridium aquilinum), MC = Purple moor-grass Molinia caerulea, and VM = European blueberry Vaccinium myrtillus.

${ }^{c}$ Effective detection distance as estimated with a point model with a half-normal detection probability function with $\log _{10}$-transformed body mass as covariate.

- represents combinations of species and habitat type that were not observed.
} 


\section{6 | Chapter 4}

Table S4.3. The relative abundance of deer (passage rate) and Ixodes ricinus tick density for the twenty forest plots in the cross-sectional study.

\begin{tabular}{|c|c|c|c|c|c|c|c|}
\hline \multirow{2}{*}{ Site } & \multicolumn{4}{|c|}{ Deer passage rate $\left(\mathrm{m}^{-1} \mathrm{~d}^{-1}\right)$} & \multicolumn{3}{|c|}{$\begin{array}{l}\text { Ixodes ricinus density } \\
\qquad\left(100 \mathrm{~m}^{-2}\right)\end{array}$} \\
\hline & All deer & Roe deer & Fallow deer & Red deer & Larvae & Nymphs & Adults \\
\hline $\begin{array}{l}\text { Amsterdamse } \\
\text { Waterleiding } \\
\text { Duinen }\end{array}$ & 0.84 & 0 & 0.84 & 0 & 349 & 60.5 & 5.3 \\
\hline Bergherbos & 0.02 & 0.02 & 0 & 0 & 39 & 72.4 & 2.7 \\
\hline Buunderkamp & 0.02 & 0.02 & 0 & 0 & 216 & 183.4 & 6.3 \\
\hline Deelerwoud & 0.05 & 0 & 0.03 & 0.03 & 265 & 137.4 & 13.4 \\
\hline $\begin{array}{l}\text { Duin en } \\
\text { Kruidberg }\end{array}$ & 0.16 & 0.01 & 0.15 & 0 & 440 & 72.5 & 4.4 \\
\hline Enkhout & 0.06 & 0.01 & 0.01 & 0.04 & 75 & 114.8 & 5.3 \\
\hline $\begin{array}{l}\text { Enkhout } \\
\text { (exclosure) }\end{array}$ & 0 & 0 & 0 & 0 & 0 & 4.0 & 2.3 \\
\hline Halfmijl & 0.06 & 0.06 & 0 & 0 & 103 & 127.6 & 4.6 \\
\hline Herperduin & 0.01 & 0.01 & 0 & 0 & 6 & 10.0 & 0.3 \\
\hline Kremboong & 0.05 & 0.05 & 0 & 0 & 187 & 66.2 & 2.3 \\
\hline Maashorst & 0.03 & 0.03 & 0 & 0 & 250 & 53.3 & 1.4 \\
\hline Pettemerduin & 0 & 0 & 0 & 0 & 0 & 10.8 & 1.6 \\
\hline $\begin{array}{l}\text { Planken } \\
\text { Wambuis }\end{array}$ & 0.04 & 0.03 & 0 & 0.01 & 131 & 63.3 & 1.7 \\
\hline Rheebruggen & 0.05 & 0.05 & 0 & 0 & 517 & 71.6 & 4.1 \\
\hline $\begin{array}{l}\text { Schoorlse } \\
\text { Duinen }\end{array}$ & 0 & 0 & 0 & 0 & 0.1 & 1.8 & 0.3 \\
\hline Stameren & 0.05 & 0.05 & 0 & 0 & 123 & 94.5 & 7.8 \\
\hline Valenberg & 0.04 & 0.003 & 0.005 & 0.03 & 230 & 63.7 & 7.7 \\
\hline Vijverhof & 0.05 & 0.05 & 0 & 0 & 46 & 86.6 & 1.9 \\
\hline Vledderhof & 0.05 & 0.05 & 0.001 & 0 & 137 & 33.7 & 2.6 \\
\hline Zwanemeerbos & 0.04 & 0.04 & 0 & 0 & 102 & 56.7 & 9.0 \\
\hline
\end{tabular}


Deer Presence Determines Sheep Tick Density | 77 


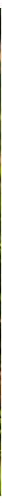

burden, your burden

is extrinsically determined

your gender, your size

nor your species otherwise

have any effect on this aspect

of your prudent life

except that you can expect

this tricky load, your burden

\section{Ronald Hofmeester}




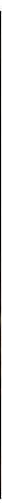

T.R. Hofmeester, P.A. Jansen, H.H.T. Prins, H. Sprong \& S.E. van Wieren

\section{Determinants of Tick (IXODES RICINUS) BURDEN ON Rodent Populations in Dutch Forests}

The number of vectors feeding on individual hosts is a key determinant in vector-borne pathogen dynamics. The vector burden on a host is in turn determined by both intrinsic (e.g., body mass and sex) and extrinsic (e.g., parasite density in the environment) factors. We assessed the relative importance of these factors by comparing the burden of the Sheep tick (Ixodes ricinus) on small rodents between twenty forest plots across the Netherlands, and relating this to species, sex and individual body mass of the rodents as intrinsic factors, and the relative abundance of deer and predators and rodent density as extrinsic factors. We found that extrinsic factors were more important for explaining tick burden than intrinsic factors. All relationships could be explained by one mechanism where the day range of rodents is the main determinant of their tick burden. Our results imply that changes in vertebrate assemblage can indirectly, via the tick burden on rodents, affect the prevalence of tick-borne pathogens. 


\section{0 | Chapter 5}

\section{Introduction}

Parasite burden - the number of parasites feeding on an individual host - is a key determinant in parasite population dynamics ${ }^{4,210}$. Which factors determine parasite load is of key interest in disease ecology and animal health, as many parasites negatively affect the condition of the host or vector pathogens. An increase in vector burden will potentially increase the number of hosts infected with a vector-borne pathogen and subsequently the number of infected vectors in the environment ${ }^{33}$, which is directly related to disease risk ${ }^{204}$.

For parasites that are encountered in the environment, parasite burdens on hosts are determined by three factors: 1) the number of parasites in the environment, and 2) the day range of the host, which together determine the encounter rate of parasites and hosts ${ }^{12}$, and 3) the resistance or tolerance of hosts towards the parasite ${ }^{13}$. The number of parasites in the environment is mainly determined by the number of host individuals that can sustain the parasite, which could either be from one species for a specialist parasite, or from multiple species for a generalist parasite ${ }^{211}$. The day range of the host relative to the presence of parasites is determined by many factors. Large host species tend to move over larger distances than small host species ${ }^{67,212}$ and males often have larger home and day ranges than females ${ }^{213}$. In addition, predation risk can decrease the distance travelled by prey species ${ }^{214}$. Finally, day range and home range size often decline with conspecific density ${ }^{213}$. The likelihood of a host individual encountering a parasite thus depends on intrinsic factors (individual characteristics of the host; e.g., species, size and sex) as well as extrinsic factors (abundance of other animals in the community). While the relationship between parasite burden and intrinsic factors has been studied in many systems ${ }^{215-217}$, the relationship with extrinsic factors is less well understood ${ }^{218}$, let alone their relative importance.

In Europe and North-America, the most common vector-borne pathogens are transmitted by ticks of the Ixodes ricinus complex ${ }^{60}$. Both I. ricinus and $I$. scapularis are three-host ticks. They feed mainly on rodents as larvae, on rodents, birds and deer as nymphs, and on deer as adults ${ }^{36,190}$ (Chapter 2). The number of larvae in the environment is related to the density and activity of deer, which determine the reproductive success of adult ticks ${ }^{32}$ (Chapter 4). Small rodents that feed these larvae are reservoirs for multiple tick-borne pathogens such as Babesia microti, Borrelia burgdorferi s.l., Borrelia miyamotoi and Candidatus 
Neoehrlichia mikurensis ${ }^{30,50}$. The rodents get infected by feeding infected larvae or nymphs, and subsequently transmit the pathogen to uninfected larvae ${ }^{36}$.

The goal of our study was to understand which factors determine the Sheep tick (I. ricinus) burden on small rodents. We measured the tick burden on three rodent species, Bank vole (Myodes glareolus), Field vole (Microtus agrestis), and Wood mouse (Apodemus sylvaticus) in 20 forest plots in the Netherlands that differed strongly in their vertebrate assemblage composition, and related these to both intrinsic and extrinsic factors. The intrinsic factors were species, sex and body mass, which are known determinants of tick burden on small mammals $32,89,95,215$. The extrinsic factors were the relative abundance of deer, the relative abundance of predators and rodent density. The predictions tested were that tick burden 1) is higher for Bank voles and Wood mice compared to Field voles, 2) is higher for male rodents than to females, 3) increases with body weight, 4) increases with the relative abundance of deer, 5) decreases with the relative abundance of predators and 6 ) decreases with rodent density.

\section{Methods}

\section{Study sites}

We collected data in twenty 1-ha forest plots scattered across the Netherlands, with a minimum distance between plots of $5 \mathrm{~km}$, except for two plots that were just 150m apart, but located inside (ENc) and outside (EN) a large 3 year old deer exclosure (Table S4.1). Eleven plots were sampled in 2013 and nine in 2014. All plots were situated within Pedunculate oak (Quercus robur), Scots pine (Pinus sylvestris) or mixed forests on sandy soils with a well-developed herbaceous plant layer (Table S4.1). Two sites, Schoorlse Duinen (SD) and Pettemerduinen (PD), were located in the northwest of the Netherlands, where no deer are present. All other sites were within the range of Roe deer (Capreolus capreolus).

\section{Rodent density and tick burden}

We used live trapping to measure rodent density and tick burden, in July and August. In each plot, we established a grid of 8x8 (64) Longworth small mammal live traps (Heslinga Traps, Groningen, the Netherlands) with a $12 \mathrm{~m}$ inter-trap distance. Traps were baited with maize, wheat, mealworms, a piece of carrot, and had hay as nesting material. We pre-baited the live traps for three days, then inspected the traps during six consecutive trapping sessions at twelve hour 


\section{2 | Chapter 5}

intervals. Every plot was sampled once. Captured rodents were transferred from the trap into a transparent plastic bag, from which we identified the animal to species $^{219}$. Shrews (Soricidae) were released immediately. Mice and voles were sexed and weighted, and the head, ears, throat and neck checked for ticks. A random selection of ticks was collected for identification to species using an established identification key 220 . We measured larval and nymphal burden (no adults were found on the rodents).

All rodents were individually marked by clipping some of the top fur in a unique pattern, which we used for individual identification when recaptured. All experimental handling procedures were approved by the Animal Experiments Committee of Wageningen University (WUR-2013055 and WUR-2014019) and by the Netherlands Ministry of Economic Affairs (FF/75A/2013/003).

We estimated the density of each rodent species using the capture-markrecapture models for closed populations presented by Otis etal. ${ }^{221}$ as implemented in $\mathrm{MARK}^{222}$, assuming that the probability of capture () and the probability of recapture () were equal and constant during trapping sessions. Because we trapped animals in an area of 1 ha, we report the abundance estimates from MARK as densities per hectare. If the number of animals captured was too low for capture-mark-recapture analysis, we used the "minimal number of individuals known alive' per hectare as the density estimate.

\section{Relative abundance of deer and predators}

We used camera trapping to estimate the relative abundance of medium-sized and large mammals during March - November, the main activity period of $I$. ricinus in the Netherlands ${ }^{156,166}$. In each plot, we placed two camera traps (HC500; RECONYX Inc., Holmen, WI, U.S.A.) on a randomly selected tree that were $>30$ $\mathrm{m}$ apart, at $40 \mathrm{~cm}$ above the ground, viewing north and parallel to the ground. Cameras were set to the highest sensitivity, to take a series of ten photographs when triggered, and with no delay between triggers. Each camera was deployed for four weeks, after which it was relocated to a new tree within the plot until we had 18 positions, totalling 504 camera trapping days per plot. Camera theft and malfunction caused some variation in the total number of camera trapping days between plots (Table S4.1). We used an automated image processing tool ${ }^{178}$ in which sequences of photographs were combined per event. Time-lapse photographs were taken every twelve hours, so that in case of camera failure, the last photograph could be used to calculate sampling effort. 


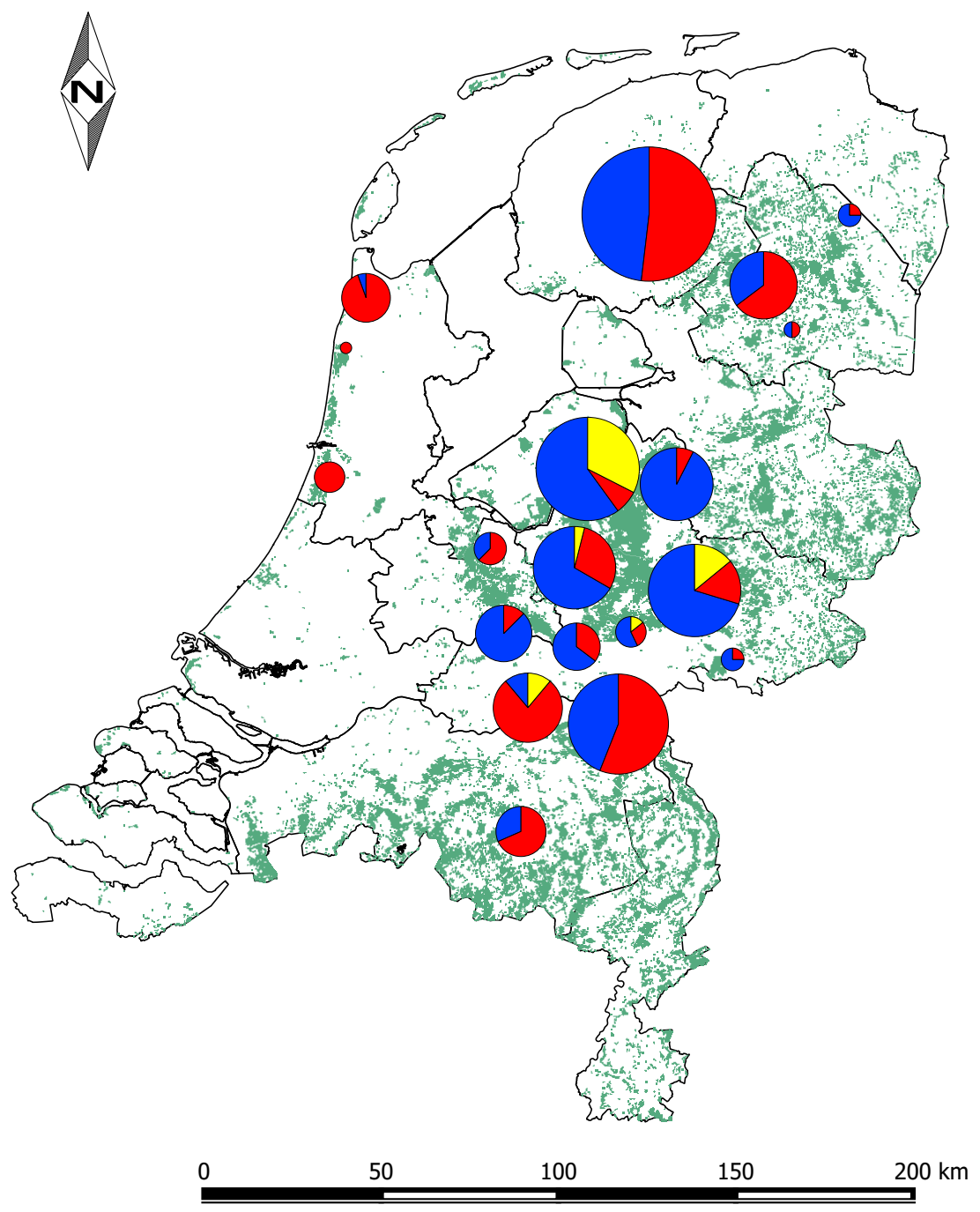

Figure 5.1. Map of the Netherlands with the locations where rodents were captured. The size of the pie chart represents the number of rodents captured, species are indicated by the colours: blue $=$ Bank vole, red $=$ Wood mouse, yellow $=$ Field vole.

To control for variation in detectability between species, we quantified the effective detection distance (EDD) for each species per habitat type using the line-transect method ${ }^{198}$ (Chapter 3 ). This involved the placement of a line of markers in the centre of the view at distance intervals of $2.5 \mathrm{~m}$. From each animal passage, we recorded the species and distance at which the animal passed between the markers. Using these data, we estimated the EDD for each species per habitat 


\section{4 | Chapter 5}

type (Table S5.1), using a model with a detection probability described by a point model with a half-normal detection probability function with $\log _{10}$ transformed body mass as covariate ${ }^{198}$ (Chapter 3 ). Body mass estimates for all species were taken from the PanTHERIA database ${ }^{179}$. The EDD estimates were used to determine passage rates per species per camera location as,

$$
P R_{i}=\frac{x_{i}}{t \cdot E D D_{i_{h}}}
$$

Equation 5.1

where $P R_{i}$ is the passage rate of species $i\left(\right.$ meter $^{1}$ day $\left.^{-1}\right), x_{i}$ the number of passages of species $i, t$ the total time at which the camera was active (in days), and $E D D_{\text {in }}$ the effective detection distance of species $i$ in habitat type $h$ (in meters). Records of animals that did not cross the line of markers were discarded. By doing so, we eliminated the need to correct for differences in detection angle between species and habitat types ${ }^{54}$. For each plot, we calculated the average passage rate $\left(\mathrm{m}^{-1}\right.$ $\left.\mathrm{d}^{-1}\right)$ per species using the arithmetic mean. The deer passage rate was then determined by summing the passage rates of all deer, and predator passage rate by summing the passage rates of all mammalian predators of rodents. For clarity we will refer to passage rates as (relative) abundance, but strictly speaking it is a combination of density and activity in a plot.

\section{Statistical analysis}

Statistical analyses were performed in $\mathrm{R}$ 3.2.381. We used hierarchical generalized linear mixed-effects models (GLMM) to test which parameters were correlated with larval and nymphal burdens on rodents, including intrinsic (individual) factors and extrinsic (plot) factors. Individual factors considered were: species, sex, and body mass of the individual rodents. We transformed the sex to a binomial variable using 0 for female and 1 for male after which we centred the parameter by extracting the mean. Rodent body mass was $\log _{10}$ transformed and then standardized by extracting the mean and dividing by two standard deviations ${ }^{223}$. Extrinsic factors considered were, deer passage rate, predator passage rate and rodent density of the forest plots. All three factors were $\log _{10}$-transformed (we added 0.01, the minimal passage rate found for deer, to accommodate the transformation for three plots that had no records of deer) and then standardized as above. All transformations were applied to approximate normal distributions for the parameters.

As variance inflation factor of all centred and standardized parameters were 
Table 5.1. Total number of rodents caught, mean prevalence of infestation $(P)$ with Ixodes ricinus larvae and nymphs, and median intensity of infestation $(I)$ with $I$. ricinus larvae and nymphs. No adults were found on the rodents. SD is standard deviation of the mean.

\begin{tabular}{lcllll}
\hline Species & $\mathrm{n}$ & $\begin{array}{l}\boldsymbol{P}_{\text {larvae }} \\
\text { Mean (SD) }\end{array}$ & $\begin{array}{l}\boldsymbol{I}_{\text {larvae }} \\
\text { Median (range) }\end{array}$ & $\begin{array}{l}\boldsymbol{P}_{\text {nymphs }} \\
\text { Mean (SD) }\end{array}$ & $\begin{array}{l}\boldsymbol{I}_{\text {nymphs }} \\
\text { Median (range) }\end{array}$ \\
\hline Bank vole & 331 & $0.86(0.35)$ & $7(1-176)$ & $0.10(0.30)$ & $1(1-5)$ \\
Field vole & 42 & $0.79(0.42)$ & $3(1-386)$ & $0.17(0.38)$ & $2(1-9)$ \\
Wood mouse & 253 & $0.84(0.37)$ & $8(1-343)$ & $0.16(0.37)$ & $1(1-8)$ \\
\hline
\end{tabular}

below 2 , indicating that collinearity was not a problem ${ }^{224}$, we used all variables in the modelling. We explored possible interactions between body mass and sex, and between species and sex and body mass, but none of the interactions was found to be significant, so the final model did not include any interactions. We modelled larval and nymphal burden with zero-altered models, using a two-step approach $^{225}$. First, we modelled the presence or absence of larvae or nymphs on an individual (prevalence of infestation: $P$ ) using a hierarchical GLMM with a binomial distribution and logit link function. Secondly, we modelled the number of larvae or nymphs, given presence (intensity of infestation: I), using a hierarchical GLMM with a zero-truncated negative binomial distribution and log link function. We dealt with differences between intrinsic and extrinsic factors by adding a random intercept per plot nested in year. This resulted in two models for larval burden and two for nymphal burden.

We fitted GLMMs using the gImmADMB package ${ }^{200,201}$. We tested the four full models and all possible sub-models using all parameters described above without interactions. We selected the best fitting models from our set by calculating AICC values and Akaike weights $\left(\omega_{m}\right)$ for the four different models using the dredge function of the MuMIn package ${ }^{82,83}$. To account for model selection uncertainty, we selected all models with $\triangle \mathrm{AICC}<4$ for model averaging, and calculated a weighted average regression coefficient and relative importance $\left(\omega_{p}\right)$ for all parameters included in the best performing models ${ }^{82,226}$.

\section{Results}

We caught a total of 626 rodents in twenty plots belonging to three species: Bank vole, Field vole and Wood mouse (Figure 5.1). One site (Duin en Kruidberg) 


\section{6 | Chapter 5}

yielded no rodents and was excluded from the analysis. We captured only one Wood mouse in Schoorlse Duinen (SD), and most rodents (135) in Vledderhof (VL; Figure 5.1). We counted a total of 10,576 larval and 158 nymphal Ixodes spp. attached to the rodents (Table 5.1). All collected ticks were identified as Ixodes ricinus. Both larval and nymphal burden varied widely between species, individuals and plots (Figure 5.2).

Camera traps recorded fourteen mammal species (Table S5.1), including three deer species - Fallow deer (Dama dama), Red deer (Cervus elaphus) and Roe deer - and five mammalian rodent-predator species - European pine marten (Martes martes), feral House cat (Felis catus), Polecat (Mustela putorius), Red fox (Vulpes vulpes) and Stone marten (Martes foina). Estimates of deer and predator passage rate and rodent density per plot are shown in Table 5.2. Deer were absent in three plots (Enkhout exclosure (ENc), Pettermerduin (PD) and $\mathrm{SD}$ ). The lowest deer passage rate was found in Herperduin (HD), and the highest

Table 5.2. Estimates of deer and predator passage rate and rodent density per plot.

\begin{tabular}{llll}
\hline \multirow{2}{*}{ Plot } & \multicolumn{2}{l}{ Passage ${\text { rate }\left(\mathrm{m}^{-1} \mathrm{~d}^{-1}\right)}^{2}$ Rodent density } \\
\cline { 2 - 3 } & Deer & Predators & \\
\cline { 1 - 2 } Amsterdamse Waterleiding & 0.844 & 0.008 & 7.2 \\
Duinen (AW) & 0.019 & 0.007 & 4 \\
Bergherbos (BB) & 0.024 & 0.004 & 18.7 \\
Buunderkamp (BU) & 0.052 & 0.004 & 68.6 \\
Deelerwoud (DW) & 0.055 & 0.012 & 48.8 \\
Enkhout (EN) & 0.000 & 0.001 & 86.2 \\
Enkhout exclosure (ENc) & 0.012 & 0.017 & 85.8 \\
Halfmijl (HM) & 0.061 & 0.002 & 29.6 \\
Herperduin (HD) & 0.050 & 0.020 & 2 \\
Kremboong (KB) & 0.031 & 0.006 & 47.1 \\
Maashorst (MH) & 0.000 & 0.023 & 19.7 \\
Pettemerduin (PD) & 0.038 & 0.009 & 7.7 \\
Planken Wambuis (PW) & 0.048 & 0.021 & 38.3 \\
Rheebruggen (RB) & 0.000 & 0.011 & 1 \\
Schoorlse Duinen (SD) & 0.049 & 0.013 & 28.2 \\
Stameren (ST) & 0.041 & 0.013 & 67.5 \\
Valenberg (VA) & 0.046 & 0.004 & 8 \\
Vijverhof (VH) & 0.046 & 0.033 & 327.7 \\
Vledderhof (VL) & 0.038 & 0.006 & 4.9 \\
Zwanemeerbos (ZM) & & & \\
\hline
\end{tabular}



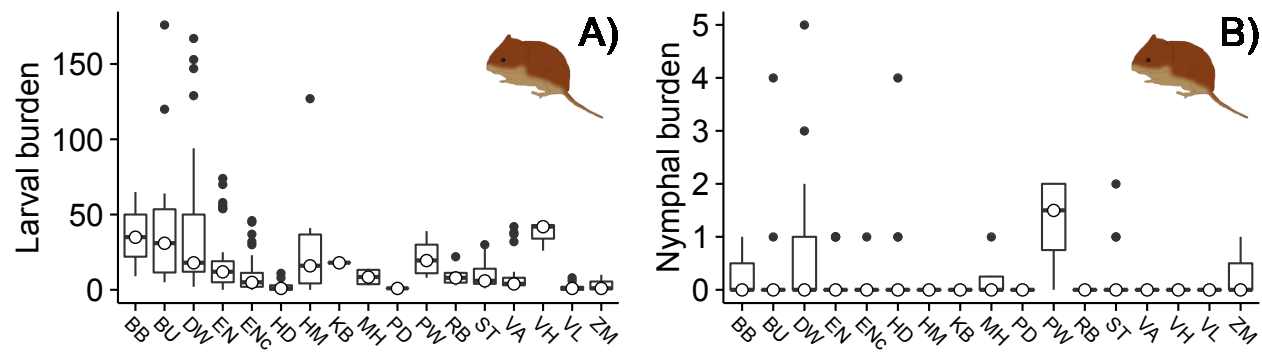

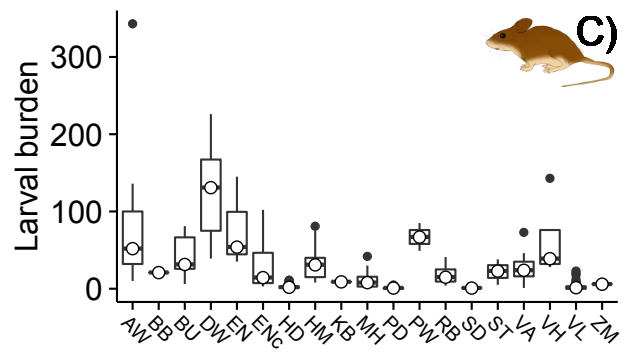

Plot

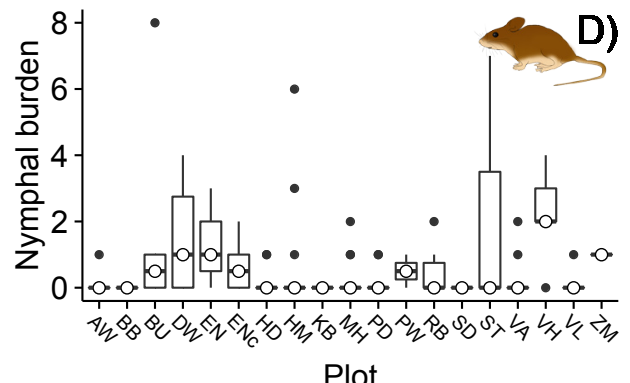

Plot

Figure 5.2. Boxplots showing variation between plots in number of $I$. ricinus A) larvae and B) nymphs parasitizing Bank voles, and C) larvae and D) nymphs parasitizing Wood mice. Central bars with white diamond are medians, boxes are interquartile ranges, whiskers are 95\% range, filled dots are estimates outside the $95 \%$ range.

deer abundance was found in Amsterdamse Waterleiding Duinen (AW). ENc had the lowest predator passage rate, while most predators were detected in $\mathrm{VL}$ (Table 5.2).

Three models of prevalence of infestation with larvae $\left(P_{\text {larvae }}\right)$ had a $\Delta \mathrm{AICc}<$ 4 (Table S5.2.1). $P_{\text {larvae }}$ increased with deer abundance, and decreased with predator abundance and rodent density (Table 5.3). Field voles had a lower $P_{\text {larvae }}$ than Bank voles and Wood mice, and males had a higher $P_{\text {larvae }}$ than females (Table 5.3). Two models of intensity of infestation with larvae $\left(I_{\text {larvae }}\right)$ had a $\Delta$ AICc $<4$ (Table S5.2.2). I larvae increased with rodent body mass and the relative abundance of deer, and decreased with predators abundance. Compared to Bank voles, Field voles had a lower $I_{\text {larvae, }}$, while Wood mice had a higher $I_{\text {larvae }}$. Males had a higher $I_{\text {larvae }}$ than females (Table 5.3).

Four models of prevalence of infestation with nymphs $\left(P_{\text {nymphs }}\right)$ had a $\triangle \mathrm{AICc}<$ 4 (Table S5.2.3). $P_{\text {nymphs }}$ increased with rodent body mass, and decreased with the relative abundance of predators and rodent density. Furthermore, males had a higher $P_{\text {nymphs }}$ than females, and Wood mice had a higher $P_{\text {nymphs }}$ than Bank voles and Field voles (Table 5.4). Nine models of intensity of infestation with 


\section{8 | Chapter 5}

nymphs $\left(I_{\text {nymphs }}\right)$ had a $\triangle \mathrm{AICc}<4$ (Table S5.2.4). $I_{\text {nymphs }}$ decreased with predator abundance only (Table 5.4).

The standardized parameter estimates were higher for extrinsic factors compared to intrinsic factors, especially for larval burden (Tables 5.2 and 5.3). Parameter estimates for extrinsic factors were four to six times higher for $P_{\text {larvae }}$ than rodent body mass and sex, and the parameter estimates for the relative abundance of deer and predators for $I_{\text {larvae }}$ were approximately three times higher than those for the intrinsic factors (Table 5.2). Intrinsic and extrinsic factors were equally important in explaining variation in $P_{\text {nymphs }}$. Only one extrinsic factor, the relative abundance of predators, explained variation in $I_{\text {nymphs }}$ (Table 5.3).

\section{Discussion}

The number of vectors feeding on an individual host is one of the key parameters in vector-borne disease dynamics ${ }^{4}$. We studied how the burden of immature lxodes ricinus, the main vector for vector-borne diseases in Europe ${ }^{60}$, on three forestdwelling rodent species was related to three intrinsic factors - rodent species, sex and body mass - and three extrinsic factors - the relative abundance of deer and predators and rodent density by comparing twenty forest plots across the Netherlands. We found that tick burden differed between species, that males in general had more ticks than females, and that heavier rodents had more ticks than lighter rodents. We also found that larval burden increased with the relative abundance of deer, and that both larval and nymphal burden decreased with the relative abundance of predators and rodent density. Burdens were correlated with extrinsic factors more strongly than with intrinsic factors (Tables 5.3 and 5.4), indicating that between-site differences in vertebrate assemblage have a larger influence on the number of ticks feeding on forest rodents than the characteristics of the rodents themselves.

Our results regarding intrinsic factors are consistent with previous studies, which showed higher tick burdens on males compared to females ${ }^{215}$, an increase in tick burden with increasing body mass of the host ${ }^{95,215}$ and higher tick burdens on Apodemus mice compared to Bank voles ${ }^{89,95}$. These differences have been attributed both to differences in day range between host individuals ${ }^{215}$ and differences in resistance against or tolerance for parasites between host species ${ }^{13,23}$. Our results regarding extrinsic factors (deer and predator abundance, and rodent density) are also consistent with previous studies, which 
Table 5.3. Standardized correlation coefficients $(\beta)$, with $95 \%$ confidence intervals $(\mathrm{Cl})$, and relative importance of all parameters obtained by model averaging $\left(\omega_{p}\right)$ of the best performing GLMMs for Ixodes ricinus larval burden.

\begin{tabular}{|c|c|c|c|c|c|c|}
\hline \multirow[b]{2}{*}{ Predictor } & \multicolumn{3}{|c|}{$\begin{array}{l}\text { Prevalence of infestation with } \\
\text { larvae }\left(\boldsymbol{P}_{\text {larvae }}\right)\end{array}$} & \multicolumn{3}{|c|}{$\begin{array}{l}\text { Intensity of infestation with } \\
\text { larvae }\left(I_{\text {larvae }}\right)\end{array}$} \\
\hline & $\beta$ & $95 \% \mathrm{Cl}^{\mathrm{C}}$ & $\omega_{p}$ & $\beta$ & $95 \% \mathrm{Cl}^{\mathrm{C}}$ & $\omega_{p}$ \\
\hline Field vole ${ }^{a}$ & -1.66 & $(-2.76--0.55)$ & 1.00 & -0.49 & $(-0.89--0.09)$ & 1.00 \\
\hline Wood mouse ${ }^{a}$ & 0.39 & $(-0.15-0.92)$ & 1.00 & 0.69 & $(0.47-0.91)$ & 1.00 \\
\hline $\operatorname{Sex}^{\mathrm{b}}$ & 0.58 & $(0.09-1.07)$ & 0.90 & 0.63 & $(0.43-0.82)$ & 1.00 \\
\hline Body mass & 0.49 & $(-0.02-0.99)$ & 0.72 & 0.43 & $(0.24-0.62)$ & 1.00 \\
\hline Deer abundance & 3.41 & $(2.33-4.48)$ & 1.00 & 1.84 & $(1.06-2.62)$ & 1.00 \\
\hline Predator abundance & -2.03 & $(-2.69--1.37)$ & 1.00 & -1.56 & $(-2.23--0.89)$ & 1.00 \\
\hline Rodent density & -1.87 & $(-2.83--0.92)$ & 1.00 & -0.39 & $(-1.22-0.43)$ & 0.35 \\
\hline
\end{tabular}

a Standardized correlation coefficients as compared to zero for bank voles.

${ }^{\mathrm{b}}$ Standardized correlation coefficient for males as compared to zero for females.

c $95 \%$ confidence intervals not containing zero $(p<0.05)$ are presented in bold.

Table 5.4. Standardized correlation coefficients $(\beta)$, with $95 \%$ confidence intervals $(\mathrm{Cl})$, and relative importance of all parameters obtained by model averaging $\left(\omega_{p}\right)$ of the best performing GLMMs for Ixodes ricinus nymphal burden.

\begin{tabular}{lcccccccc}
\hline & \multicolumn{3}{c}{$\begin{array}{c}\text { Prevalence of infestation with } \\
\text { nymphs }\left(\boldsymbol{P}_{\text {nymphs }}\right)\end{array}$} & & \multicolumn{3}{c}{$\begin{array}{c}\text { Intensity of infestation with } \\
\text { nymphs }\left(I_{\text {nymphs }}\right)\end{array}$} \\
\cline { 2 - 4 } Predictor & $\beta$ & $95 \% \mathrm{Cl}^{\mathrm{c}}$ & $\omega_{\mathrm{p}}$ & & $\beta$ & $95 \% \mathrm{Cl}^{\mathrm{c}}$ & $\omega_{\mathrm{p}}$ \\
\hline Field vole $^{\mathrm{a}}$ & -0.34 & $(-1.53-0.85)$ & 1.00 & & 0.84 & $(-0.70-2.38)$ & 0.06 \\
Wood mouse & 1.34 & $(0.61-2.07)$ & 1.00 & & 0.50 & $(-0.40-1.40)$ & 0.06 \\
Sex & 1.37 & $(0.74-1.99)$ & 1.00 & & 0.64 & $(-0.47-1.74)$ & 0.36 \\
Body mass & 1.82 & $(1.07-2.56)$ & 1.00 & & 0.23 & $(-0.85-1.32)$ & 0.16 \\
Deer abundance & 0.32 & $(-0.83-1.47)$ & 0.30 & & 0.34 & $(-1.28-1.97)$ & 0.16 \\
Predator abundance & -0.95 & $(-1.89--0.00)$ & 0.71 & & -1.37 & $(-2.50--0.24)$ & 1.00 \\
Rodent density & -2.25 & $(-3.57--0.93)$ & 1.00 & & -0.09 & $(-1.75-1.56)$ & 0.15 \\
\hline
\end{tabular}

a Standardized correlation coefficients as compared to zero for bank voles.

${ }^{\mathrm{b}}$ Standardized correlation coefficient for males as compared to zero for females.

${ }^{c}$ 95\% confidence intervals not containing zero $(p<0.05)$ are presented in bold. 


\section{0 | Chapter 5}

found an increase in larval burden on rodents with increasing deer density ${ }^{32}$ and a decreasing larval burden with increasing rodent density ${ }^{95,227}$. However, we are the first to report a decrease in tick burden with increasing relative abundance of predators.

To our knowledge, our study is the first to simultaneously consider intrinsic factors and abundance of different vertebrate species as determinants of tick burden on rodents. We found that extrinsic factors explain tick burden better than do intrinsic factors. This implies that differences in vertebrate assemblage structure, measured as the relative abundance of deer and predators and rodent density, are important causes of spatial differences in I. ricinus burden on rodents. For deer abundance, this relationship is straightforward because more deer can feed more adult ticks, resulting in a larger number of larvae in the environment ${ }^{32}$ (Chapter 4 ). This is reflected by both a higher prevalence and a higher intensity of infestation with larvae on rodents in areas with high deer abundance (Table 5.3). However, for rodent density and predator abundance, the relationship is less straightforward.

We found that tick burden on rodents decreased with rodent density. Two different mechanisms can explain this relationship. First, an increase of rodent density could result in the dilution of the available nymphs and larvae over more rodents. Secondly, the day range - the product of activity level and movement speed - of rodents could decrease with rodent density ${ }^{213}$, resulting in a lower encounter rate of rodents and ticks, hence a lower prevalence and intensity of infestation. Studies investigating behavioural responses of rodents to crowding in relation to tick burdens are needed to determine which mechanism is more important.

We found a clear negative correlation between tick burden and the relative abundance of generalist mammalian predators. At least two different mechanisms may have driven this relationship. First, the likelihood of rodents being predated may increase with their tick burden, which would result in a more uniform distribution of tick burdens in sites with high predator abundance. We consider this unlikely, as we found an over-dispersed distribution of larvae on rodents for all sites, including those with the highest relative abundance of predators. Second, rodents can reduce their activity level, and thus day range in response to predation risk ${ }^{214}$, even to perceived predation risk ${ }^{228,229}$. Thus, it seems plausible that predators reduce the encounter rate of rodents and ticks. 
Although several alternative mechanisms may cause the relationships that we observed, all involve the encounter probability of rodents and ticks. A change in encounter probability due to differences in vertebrate assemblage composition is also one of the proposed mechanisms underlying the dilution effect hypothe$\mathrm{sis}^{31}$. We see a clear need for studies that explore the role of movement behaviour of hosts as explanation for differences in parasite burden between populations, and ultimately disease dynamics.

Our study is among the first to use camera traps to assess the abundance of large vertebrate hosts in a study of parasite burden (but see LoGiudice et al. ${ }^{230}$ ). Camera traps are a reliable method for measuring the relative abundance of species provided that sampling is rigorously standardized ${ }^{167}$ and that differences in sampling effort and detection distance are accounted for ${ }^{54}$, as was the case in our study. Yet, our survey was imperfect in detecting carnivores. We may not have photographed any small mammalian predators such as Weasels (Mustela nivalis) or Stoats (M. erminea) due to these species' small size and high speed, hence low detectability ${ }^{54}$. Likewise, we missed activity of avian predators such as owls and raptors, due to a combination of good thermal isolation and very little movement on the ground ${ }^{231}$. Yet, our estimates of deer and medium-sized carnivore abundance overall have a level of precision not attained by any prior study of tick burden.

Concluding, differences in the relative abundance of deer and predators and rodent density can explain the large differences in the I. ricinus burden on rodents between populations. All relevant factors appear to be related to movement behaviour changing the encounter rate between ticks and rodents. Effects of other hosts in an assemblage on the behaviour of reservoir hosts should be taken into account by researchers investigating the relationship between host and pathogen ecology, especially for vector-borne pathogens. 


\section{2 | Chapter 5}

\section{Appendix to Chapter 5}

Table S5.1. Estimated Effective Detection Distances (EDD), for the medium-sized to large mammal species photographed with camera traps, per habitat type.

\begin{tabular}{|c|c|c|c|c|c|c|}
\hline \multirow{2}{*}{ Species } & \multirow{2}{*}{$\begin{array}{l}\text { Body mass } \\
\text { (kg) }\end{array}$} & \multicolumn{5}{|c|}{$\operatorname{EDD}(m)$ by Habitat ${ }^{\mathrm{a}}$} \\
\hline & & $\mathrm{CE}^{\mathrm{C}}$ & $\mathrm{DF}^{\mathrm{c}}$ & $\mathrm{FE}^{\mathrm{c}}$ & $\mathrm{MC}^{\mathrm{C}}$ & $\mathrm{VM}^{\mathrm{c}}$ \\
\hline $\begin{array}{l}\text { Eurasian red squirrel } \\
\text { (Sciurus vulgaris) }\end{array}$ & 0.3 & 4.0 & 3.0 & 2.7 & 2.5 & 2.4 \\
\hline $\begin{array}{l}\text { European badger } \\
\text { (Meles meles) }\end{array}$ & 11.8 & - & 5.4 & 5.4 & 5.5 & 4.5 \\
\hline $\begin{array}{l}\text { European hare } \\
\text { (Lepus europaeus) }\end{array}$ & 3.8 & 4.9 & 4.5 & 4.3 & 4.3 & 3.7 \\
\hline $\begin{array}{l}\text { European hedgehog } \\
\text { (Erinaceus europaeus) }\end{array}$ & 0.8 & 4.3 & - & 3.2 & 3.0 & - \\
\hline $\begin{array}{l}\text { European pine marten } \\
\text { (Martes martes) }\end{array}$ & 1.3 & 4.5 & 3.7 & 3.5 & 3.4 & 3.0 \\
\hline $\begin{array}{l}\text { European Rabbit } \\
\text { (Oryctolagus cuniculus) }\end{array}$ & 1.6 & - & 3.8 & - & 3.5 & - \\
\hline $\begin{array}{l}\text { Fallow deer } \\
\text { (Dama dama) }\end{array}$ & 57.2 & 6.2 & - & 7.2 & - & 5.9 \\
\hline $\begin{array}{l}\text { Feral house cat } \\
\text { (Felis catus) }\end{array}$ & 2.9 & - & 4.3 & 4.1 & 4.0 & - \\
\hline $\begin{array}{l}\text { Polecat } \\
\text { (Mustela putorius) }\end{array}$ & 1.0 & 4.4 & - & 3.4 & 3.2 & - \\
\hline $\begin{array}{l}\text { Red deer } \\
\text { (Cervus elaphus) }\end{array}$ & 240.9 & - & - & - & - & 7.6 \\
\hline $\begin{array}{l}\text { Red fox } \\
\text { (Vulpes vulpes) }\end{array}$ & 4.8 & 5.0 & 4.6 & 4.5 & 4.5 & 3.8 \\
\hline $\begin{array}{l}\text { Roe deer } \\
\text { (Capreolus capreolus) }\end{array}$ & 22.5 & 5.7 & 6.0 & 6.1 & 6.3 & 5.0 \\
\hline $\begin{array}{l}\text { Stone marten } \\
\text { (Martes foina) }\end{array}$ & 1.7 & - & 3.9 & 3.7 & 3.6 & - \\
\hline $\begin{array}{l}\text { Wild boar } \\
\text { (Sus scrofa) }\end{array}$ & 84.5 & - & - & - & - & 6.3 \\
\hline
\end{tabular}

${ }^{a}$ The abbreviations are related to the dominant species of vegetation in the herbaceous layer in the 1 hectare forest plot. $\mathrm{CE}=$ Calamagrostis epigejos, $\mathrm{DF}=$ Deschampsia flexuosa, $\mathrm{FE}=$ fern species $($ Dryopteris dilatata, Polypodium vulgare or Pteridium aquilinum), $\mathrm{MC}=$ Molinia caerulea, and VM = Vaccinium myrtillus .

${ }^{\mathrm{b}}$ Body mass values are taken from the PanTHERIA database ${ }^{179}$.

${ }^{\mathrm{C}}$ Estimates of EDD are based on a point model with a half-normal detection probability function with $\log _{10^{-}}$ transformed body mass as covariate.

- represents combinations of species and habitat that were not observed. 
Table S5.2.1. Model results for the analysis of prevalence of infestation with larvae $\left(P_{\text {larvae }}\right)$. Models presented are the best performing hierarchical GLMMs with binomial distribution with logit link function.

\begin{tabular}{llll}
\hline & \multicolumn{3}{c}{ Model } \\
\cline { 2 - 4 } Predictor & 1 & 2 & 3 \\
\hline Field vole $^{\mathrm{a}}$ & -1.70 & -1.53 & -1.73 \\
Wood mouse $^{\mathrm{a}}$ & 0.39 & 0.39 & 0.38 \\
Deer abundance & 3.44 & 3.39 & 3.27 \\
Rodent density & -1.88 & -1.88 & -1.80 \\
Predator abundance & -2.03 & -2.04 & -2.02 \\
Sex & 0.59 & 0.57 & - \\
Weight $^{\mathrm{b}}$ & 0.49 & - & 0.47 \\
AlCc $^{c}$ & 0.00 & 1.56 & 3.55 \\
$\omega_{m}{ }^{c}$ & 0.61 & 0.28 & 0.10 \\
\hline
\end{tabular}

a Standardized correlation coefficients as compared to zero for bank voles.

${ }^{\mathrm{b}}$ Standardized correlation coefficient for males as compared to zero for females.

${ }^{c}$ Akaike weight as calculated for for each model ${ }^{226}$.

- Parameter was not included in the model.

Table S5.2.2. Model results for the analysis of intensity of infestation with larvae $\left(I_{\text {larvae }}\right)$. Models presented are the best performing hierarchical GLMMs with truncated negative binomial distribution and log link function.

\begin{tabular}{lll}
\hline & \multicolumn{2}{c}{ Model } \\
\cline { 2 - 3 } Predictor & 1 & 2 \\
\hline Field vole $^{\mathrm{a}}$ & -0.49 & -0.49 \\
Wood mouse $^{\mathrm{a}}$ & 0.69 & 0.69 \\
Deer abundance & 1.87 & 1.78 \\
Predator abundance & -1.58 & -1.53 \\
Sex & 0.63 & 0.63 \\
Weight & 0.43 & 0.43 \\
Rodent density & - & -0.39 \\
$\Delta$ AlCc & 0.00 & 1.27 \\
$\omega_{m}{ }^{\mathrm{b}}$ & 0.65 & 0.35 \\
\hline
\end{tabular}

a Standardized correlation coefficients as compared to zero for bank voles.

${ }^{\mathrm{b}}$ Standardized correlation coefficient for males as compared to zero for females.

${ }^{c}$ Akaike weight as calculated for for each model ${ }^{226}$.

- Parameter was not included in the model. 


\section{4 | Chapter 5}

Table S5.2.3. Model results for the analysis of prevalence of infestation with nymphs $\left(P_{\text {nymphs }}\right)$. Models presented are the best performing hierarchical GLMMs with binomial distribution with logit link function.

\begin{tabular}{lllll}
\hline & \multicolumn{5}{c}{ Model } \\
\cline { 2 - 5 } Predictor & 1 & 2 & 3 & 4 \\
\hline Field vole $^{\mathrm{a}}$ & -0.39 & -0.37 & -0.23 & -0.21 \\
Wood mouse $^{\mathrm{a}}$ & 1.36 & 1.33 & 1.32 & 1.32 \\
Sex $^{\mathrm{b}}$ & 1.36 & 1.37 & 1.37 & 1.37 \\
Weight & 1.83 & 1.82 & 1.80 & 1.79 \\
Rodent density & -2.25 & -2.17 & -2.33 & -2.29 \\
Predator abundance & -0.94 & -0.97 & - & - \\
Deer abundance & - & 0.37 & - & 0.17 \\
$\Delta$ AlCc & 0.00 & 1.63 & 1.64 & 3.62 \\
$\omega_{\mathrm{m}}{ }^{\mathrm{c}}$ & 0.49 & 0.22 & 0.22 & 0.08 \\
\hline
\end{tabular}

a Standardized correlation coefficients as compared to zero for bank voles.

${ }^{\mathrm{b}}$ Standardized correlation coefficient for males as compared to zero for females.

${ }^{c}$ Akaike weight as calculated for for each model ${ }^{226}$.

- Parameter was not included in the model.

Table S5.2.4. Model results for the analysis of intensity of infestation with nymphs $\left(P_{\text {nymphs }}\right)$. Models presented are the best performing hierarchical GLMMs with truncated negative binomial distribution and log link function.

\begin{tabular}{llllllllll}
\hline & \multicolumn{1}{c}{ Model } \\
\cline { 2 - 10 } Predictor & 1 & 2 & 3 & 4 & 5 & 6 & 7 & 8 & 9 \\
\hline Predator abundance & -1.34 & -1.48 & -1.28 & -1.38 & -1.34 & -1.11 & -1.51 & -1.43 & -1.50 \\
Sex & - & 0.63 & - & - & - & - & 0.64 & 0.63 & 0.67 \\
Weight & - & - & 0.23 & - & - & - & - & 0.24 & - \\
Deer abundance & - & - & - & 0.31 & - & - & 0.40 & - & -0.25 \\
Rodent density & - & - & - & - & 0.003 & - & - & - & - \\
Field vole & - & - & - & - & - & 0.84 & - & - & - \\
Wood mouse $^{\mathrm{b}}$ & - & - & - & - & - & 0.50 & - & - & - \\
$\Delta$ AlCc $^{\mathrm{c}}$ & 0.00 & 0.94 & 2.17 & 2.20 & 2.35 & 3.07 & 3.13 & 3.16 & 3.27 \\
$\omega_{\mathrm{m}}{ }^{\mathrm{b}}$ & - & 0.18 & 0.10 & 0.10 & 0.09 & 0.06 & 0.06 & 0.06 & 0.06 \\
\hline
\end{tabular}

a Standardized correlation coefficient for males as compared to zero for females.

${ }^{\mathrm{b}}$ Standardized correlation coefficients as compared to zero for bank voles.

${ }^{c}$ Akaike weight as calculated for for each model ${ }^{226}$.

- Parameter was not included in the model. 
Determinants of Tick Burden on Rodents | 95 


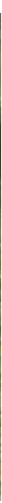

mice think twice

a carnivore will take you by surprise

and for living with deer

you'll pay the price

so roll the dice or be precise

and rate the risk

of a tick getting sick

because you behave unwise

Ronald Hofmeester 


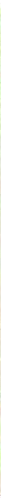

T.R. Hofmeester, P.A. Jansen, H.J. Wijnen, E.C. Coipan, M. Fonville, H.H.T. Prins, H. Sprong \& S.E. van WIEREN

\section{Cascading Effects of Changes in Vertebrate Assemblages on Rodent-transmitted TICK-BORNE DISEASE RISK}

The density of infected nymphs (DIN) is an often used measure of tick-borne disease risk. We tested three alternative mechanisms by which vertebrate assemblage composition can affect DIN - 1) encounter reduction between individual hosts and (infected) ticks, 2) vector augmentation by additional hosts feeding extra ticks, and 3) regulation of reservoir host populations by predators - in a system with three rodent-transmitted tick-borne pathogens. We compared the density of Ixodes ricinus nymphs infected with Borrelia afzelii, Borrelia miyamotoi and Candidatus Neoehrlichia mikurensis between twenty forest sites across the Netherlands, and correlated those to the relative abundance of deer and predators as measured by camera trapping, rodent density as measured by live trapping, and median larval burden on rodents, using path analysis. We found support for vector augmentation by deer, and for encounter reduction between immature $I$. ricinus and rodents by conspecifics and generalist mammalian predators. Larval burden on rodents was positively correlated with DIN for all three pathogens. We did not find support for reservoir host regulation by predators, nor a correlation between reservoir host density and DIN. Our results imply that changes in vertebrate assemblages can lead to cascading effects on rodent-transmitted tick-borne disease risk, via larval burden on rodents. 


\section{8 | Chapter 6}

\section{Introduction}

The incidence of zoonotic vector-borne diseases has increased in recent decades ${ }^{2}$. In Europe and North America, the most common vector-borne pathogens are transmitted by two tick species from the Ixodes ricinus complex ${ }^{60}:$ I. ricinus in Europe and I. scapularis in North America. Both species are three-host ticks, mainly feeding on small rodents as larvae, on multiple host species as nymphs, and on deer as adults ${ }^{36,190}$ (Chapter 2). While feeding on these hosts, ticks can become infected with pathogens that are transmitted by the host ${ }^{8}$. Infection prevalence in ticks often increases with the number of bloodmeals, thus larvae generally have a lower infection prevalence than nymphs, which in turn have a lower infection prevalence than adults ${ }^{41}$. Population densities of ticks show an opposite pattern, larvae being more abundant than nymphs, which in turn are more abundant than adult ticks ${ }^{8}$. Therefore, the density of infected nymphs (DIN) is often referred to as the most important ecological parameter that, together with the level of human exposure to ticks, determines tick-borne disease risk ${ }^{39}$. The DIN is determined by two parameters: 1) the density of nymphs and 2) the infection prevalence in these nymphs.

There are large differences in nymphal infection prevalence (NIP) between areas for many tick-borne pathogens such as Borrelia burgdorferi s.l., the causative agent of Lyme borreliosis ${ }^{41}$, Borrelia miyamotoi, the causative agent of acute febrile illness ${ }^{43}$, Anaplasma phagocytophilum, the causative agent of human granulocytic anaplasmosis ${ }^{42}$ and Candidatus Neoehrlichia mikurensis, the causative agent of neoehrlichiosis ${ }^{44}$, resulting in large differences between areas in disease risk for these pathogens. The mechanisms behind these differences are poorly understood, but it is widely assumed that they are related to differences in vertebrate host composition ${ }^{157}$.

There are several possible mechanisms by which different vertebrate host species could influence NIP or DIN ${ }^{31}$. For tick-borne pathogens, the most likely mechanisms are: 1) encounter reduction, 2) vector augmentation, and 3) reservoir host regulation. Encounter reduction is a decrease in the chance of a reservoir host individual encountering a tick, which may result from a decreased activity of a reservoir host due to the presence of (conspecific) competitors and predators, leading to lower tick burden ${ }^{31}$. Vector augmentation is an increase in tick density, resulting from the presence of maintenance hosts, such as deer ${ }^{190}$ (Chapters $2 \& 4$ ), leading to a higher tick burden on reservoir-competent hosts. 
An increase in individual tick burden can lead to an increase in DIN as it increases the number of infected ticks (nymphs and adults) feeding on an individual host, which increases the number of infected hosts. Subsequently, if the number of larvae feeding on each infected host increases, so will the absolute number of larvae that become infected, resulting in a higher DIN ${ }^{33}$. Reservoir host regulation, finally, refers to predators reducing the population density of reservoir hosts through predation ${ }^{31,160}$. This, theoretically, results in a decreased DIN due to a decrease in reservoir-host availability for ticks ${ }^{33}$. Therefore, changes in the presence or abundance of predators could have cascading effects on DIN by affecting both the density of, and the tick burden on reservoir hosts ${ }^{160}$. Evidence for these mechanisms is sparse. In Europe, where rodents are important reservoir hosts for several tick-borne pathogens ${ }^{50}$, two studies have linked some variation in rodent tick-burden to variation in deer density (positive correlation) ${ }^{32}$ and rodent density (negative correlation) ${ }^{95}$, but left a large part of the variation unexplained. Neither study considered predators of rodents.

In this study, we aimed to find empirical evidence for encounter reduction, vector augmentation and reservoir host regulation. Our approach was to compare the I. ricinus burden on three rodent species - Bank vole (Myodes glareolus), Field vole (Microtus agrestis), and Wood mouse (Apodemus sylvaticus) - and differences in DIN for three rodent-transmitted tick-borne pathogens - B. afzelii, B. miyamotoi and $\mathrm{Ca}$. N. mikurensis ${ }^{50}$ - between twenty forest plots that differed in their vertebrate assemblage composition. Based on the theoretical mechanisms of Keesing et al. ${ }^{31}$ and the empirical evidence of Cagnacci et al. ${ }^{32}$,and Kiffner et al. ${ }^{95}$, we propose six relationships between predator, deer and rodent density, tick burden on rodents and DIN for rodent-transmitted tick-borne pathogens (Figure 6.1): 1) tick burden on rodents increases with the density of deer, important maintenance hosts for adult female I. ricinus that can therefore increase the number of immature ticks in the environment ${ }^{32,190}$ (vector augmentation); 2) tick burden on rodents decreases with rodent density because ticks are distributed over more individuals and because rodents decrease their spatial activity, decreasing encounter rate ${ }^{95,213}$ (encounter reduction); 3 ) tick burden on rodents decreases with predator density because rodents decrease their activity in response to predation risk, decreasing encounter rate ${ }^{214}$ (encounter reduction); 4) rodent density decreases with predator density (reservoir host regulation) ${ }^{31,160 ;}$ 5) the DIN of rodent-transmitted tick-borne pathogens increases with per-capita tick burden on rodents, because the chance of individuals becoming infected with 


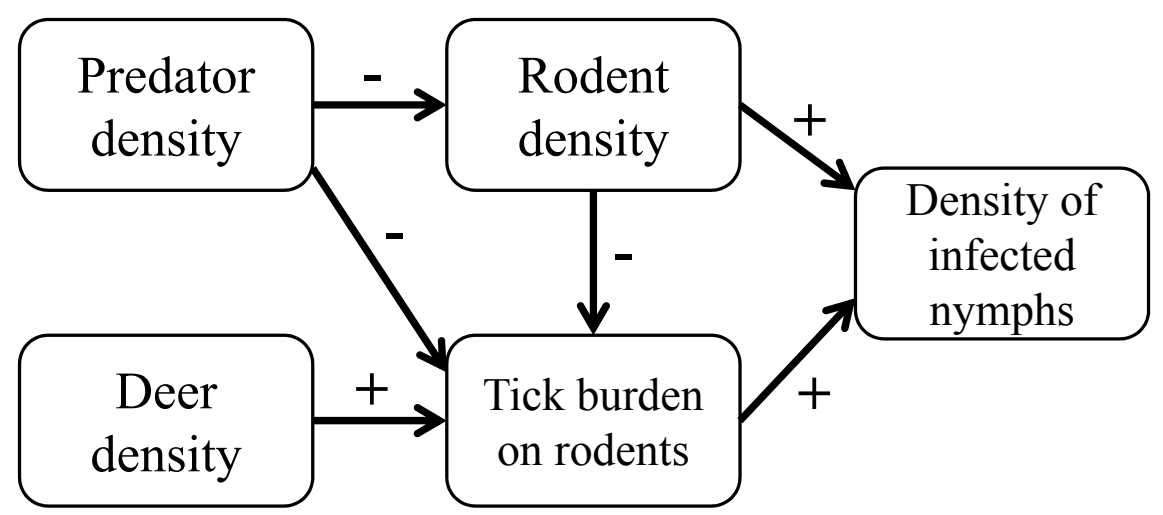

Figure 6.1. Conceptual model of relationships between vertebrate assemblage composition, tick burden on rodents and the density of infected nymphs. Reasoning and references for the hypothesized correlations are given in the main text.

a pathogen by feeding an infected nymph increases with tick burden, and each infected individual will infect more larvae, resulting in a higher DIN irrespective of rodent density; and 6) for a given per-capita tick burden, the DIN of rodenttransmitted tick-borne pathogens increases with rodent density ${ }^{33}$.

\section{Methods}

\section{Study sites}

We collected data in twenty forest plots of 1 hectare (ha) located within nineteen forest sites in the Netherlands, with $>5 \mathrm{~km}$ between sites. Sites were selected to form a large gradient in deer and predator abundance based on distribution maps and information from the managers of the nature reserves. We sampled eleven plots in 2013, and nine in 2014 (Table S4.1). We identified the dominant herbaceous species in the vegetation layer, and distinguished five types: Calamagrostis epigejos dominated, Deschampsia flexuosa dominated, fern (Dryopteris dilatata, Polypodium vulgare or Pteridium aquilinum) dominated, Molinia caerulea dominated, and Vaccinium myrtillus dominated vegetation (Table S4.1). In one site, Enkhout, we collected data in two plots $150 \mathrm{~m}$ apart, of which one was inside an exclosure of 3 ha. The exclosure was built to exclude large herbivores three years before field collection, and used by us to mimic a situation in which all larger mammals were absent, which we verified with camera trapping data. 


\section{Relative abundance of deer and predators}

Our prediction of a positive correlation between deer density and tick burden on rodents is based on the assumption that adult ticks have an increased chance to find a host when there are more deer per surface area. The number of deer passing a location can therefore be used to measure the likelihood of adult ticks to encounter deer. Similarly, behavioural changes of rodents are determined by the local density or activity of predators in a site, which can be measured by the number of predators passing a location. Therefore, passage rates of deer and predators, as measures of relative abundance ${ }^{166}$, can be used to test our predictions.

We measured passage rates of deer and predators using camera traps (HC500; Reconyx Inc., Holmen, WI, U.S.A.) during March - November, the period in which Ixodes ricinus is most active in the Netherlands ${ }^{156}$. In each plot, two camera traps were placed on two randomly selected trees, $>30$ m apart, and 40 $\mathrm{cm}$ above the ground facing north, with the view parallel to the ground. Every four weeks, both cameras were relocated to a new location within the plot, until we had 18 positions, totalling 504 camera trapping days per plot. Theft and camera malfunction caused some variation in the total number of camera trapping days per plot (Table S4.1). To quantify effective detection distance (see below), we placed a line of markers in the centre of the view of each camera, at distance intervals of $2.5 \mathrm{~m}$.

We set the cameras to the highest sensitivity, to take a series of ten photographs when triggered, and to take a new series of ten photographs as soon as they were triggered again after the first series with no delay. We used a custommade photo processing tool ${ }^{178}$ in which series of photographs that were taken within five minutes were automatically combined with the previous series of photographs. Series of photographs that were taken more than 15 minutes apart were automatically stored as separate sequences, and series of photographs separated by five to 15 minutes were checked manually to determine if it was the same animal passing, and either combined with the previous series, or stored as a separate sequence ${ }^{178}$. Time-lapse pictures were taken every 12 hours to check camera functioning, and photographs were taken when the camera was deployed and when the camera was collected. The first and last photograph taken by each camera was used to calculate the sampling time of the camera.

For each photo sequence, we recorded all animals that crossed the line of markers in the middle of the view, ignoring all other animals. By doing so, 


\section{2 | Chapter 6}

we eliminated the need to correct for differences in detection angle between species and habitat types ${ }^{198}$ (Chapter 3 ). For each of the animals that passed the line, we recorded the species and distance at which the animal passed between the markers. Using these data, we estimated the effective detection distance (EDD) for each species per habitat type (Table S5.1), using a point model with a detection probability described by a half-normal function with $\log _{10}$-transformed body mass as covariate to correct for differences in sensor sensitivity between species and habitat types ${ }^{198}$. The EDD estimates were used to determine passage rates per species per camera location as,

$$
P R_{i}=\frac{x_{i}}{t \cdot E D D_{i_{h}}}
$$

Equation 6.1

where $P R_{i}$ is the passage rate of species $i\left(\right.$ meter $^{-1}$ day $\left.^{-1}\right), x_{i}$ the number of passages of species $i, t$ the total time at which the camera was active (in days), and $E D D_{\text {in }}$ the effective detection distance of species $i$ in habitat type $h$ (in meters). This passage rate is an index of density and activity, described as the number of animals passing the camera per day standardized for differences in detection probability between species and habitats. For clarity we will refer to passage rates as (relative) abundance.

For each plot, we calculated the plot-specific passage rate per species as the arithmetic mean of the passage rates for all deployments per species. The deer passage rate $\left(\mathrm{m}^{-1} \mathrm{~d}^{-1}\right)$ per plot was calculated as the summed rates of Fallow deer (Dama dama), Red deer (Cervus elaphus) and Roe deer (Capreolus capreolus). The predator passage rate $\left(\mathrm{m}^{-1} \mathrm{~d}^{-1}\right)$ per plot was calculated as the summed rates of European pine marten (Martes martes), Feral house cat (Felis catus), Polecat (Mustela putorius), Red fox (Vulpes vulpes) and Stone marten (Martes foina).

\section{Rodent density and tick burden}

We determined tick burden per plot as: 1) the median number of larvae feeding on an individual rodent (larval burden) and 2) the average prevalence of nymphs on an individual rodent (nymphal prevalence). We used these measures to distinguish between the chance that individual rodents become infected by feeding an infected nymph (nymphal prevalence) and the chance that uninfected larvae become infected by feeding on an infected rodent (larval burden). Rodent density, larval burden and nymphal prevalence were quantified by live trapping. In each plot, we established a grid of $8 \times 8$ (64) Longworth small mammal live 
traps (Heslinga Traps, Groningen, the Netherlands) with a $12 \mathrm{~m}$ inter-trap distance, in July or August. We baited the traps with maize, wheat, mealworms, and a piece of carrot, and added hay as isolating material. We pre-baited the live traps for three days, and then checked the traps during six consecutive trapping sessions at twelve hour intervals. Captured mice were transferred from the trap into a transparent plastic bag, from which we identified the animal to species ${ }^{219}$. Shrews (Soricidae) were released immediately. We handled the mice and voles with care, by holding them by the scruff of their neck and counted all the ticks on the head, ears, throat, and neck of the animal. Median larval burden on rodents was estimated in each plot by counting all larvae on each rodent during the first capture, and taking the median as a plot-level estimate over all rodents caught in a plot. Nymphal prevalence was calculated as the percentage of rodents that were infested with one or more nymphs at first capture per plot. We collected a random selection of ticks for identification to species level in the lab using an established identification key ${ }^{220}$. All rodents were individually marked by clipping some of the top fur in a unique pattern, for individual identification when recaptured. All experimental handling procedures were approved by the Animal Experiments Committee of Wageningen University (WUR-2013055 and WUR-2014019) and by the Netherlands Ministry of Economic Affairs (FF/75A/2013/003).

We estimated the density of each rodent species using the capturemark-recapture models for closed populations presented by Otis et al. ${ }^{221}$ as implemented in MARK ${ }^{222}$, assuming that the probability of capture $(p)$ and the probability of recapture (c) were equal and constant during trapping sessions. We report the abundance estimates from MARK as densities per hectare. Where the minimum number of animals caught per species was too low to estimate a density using MARK, we used the minimal number of individuals known alive per hectare as the density estimate. For each plot, we combined the densities of the different species to calculate the overall rodent density $\left(\mathrm{ha}^{-1}\right)$.

\section{Density of infected nymphs}

To determine the density of infected nymphs with rodent-transmitted tick-borne pathogens, we collected ticks six times in each plot, once every four weeks from April - September, by blanket-dragging of twenty $10 \mathrm{~m}$ transects using a $1 \mathrm{~m}^{2}$ cotton cloth ${ }^{199}$, totalling $1200 \mathrm{~m}^{2}$. We dragged for ticks only in optimal conditions: on dry days, with air temperature $>10^{\circ} \mathrm{C}^{8}$, and in dry vegetation $<60 \mathrm{~cm} \mathrm{high}{ }^{40}$. During each session, dragging was performed within five days in all plots to 


\section{4 | Chapter 6}

minimize variation in weather conditions. We calculated the average number of nymphs per $100 \mathrm{~m}^{2}$ to analyse differences between plots. All I. ricinus nymphs were collected in Eppendorf tubes and stored at $-20^{\circ} \mathrm{C}$ until pathogen analysis.

To determine pathogen prevalence in all individual nymphs, we performed DNA extraction by alkaline lysis in ammonium hydroxide ${ }^{232}$. For the detection of $B$. burgdorferi s.l. and B. miyamotoi we performed a triplex real-time polymerase chain reaction ( $\mathrm{qPCR}$ ), targeting ospA and flaB genes. The sequences of primers and probes, and the qPCR settings are given in Appendix S6.1. We tested for the presence of $\mathrm{Ca}$. N. mikurensis DNA in a duplex $\mathrm{qPCR}^{233}$.

For typing to the genospecies level of $B$. burgdorferi s.l., the positive samples of the qPCR were further submitted to PCR targeting the variable 5S-23S intergenic spacer region (IGS), according to the protocol described in Coipan et al. ${ }^{234}$. We sequenced PCR products using an ABI PRISM BigDye Terminator Cycle sequencing Ready Reaction kit (Perkin Elmer, Applied Biosystems), confirmed the sequences by sequencing both strands ${ }^{235}$, and identified the Borrelia genospecies based on the DNA sequence of IGS. IGS sequences were stored and analysed in BioNumerics version 7.0 (Applied Math, Belgium), and assigned to Borrelia genospecies based on sequence identity with reference DNA sequences from GenBank. We determined the number of nymphs found with a co-infection of two pathogens and co-infection with all three pathogens, and estimated the overall prevalence of these co-infections using the sequencing and qPCR results.

Because only $44 \%$ of the qPCR positive nymphs yielded a successful sequence, we approximated the infection prevalence of nymphs with $B$. afzelii for each plot as,

$$
N I P_{B a}=\frac{P_{B a} \cdot I_{B b}}{N_{t}}
$$

Equation 6.2

where $N I P_{B a}$ is the nymphal infection prevalence with $B$. afzelii, $P_{B a}$ is the proportion of successful sequences identified as $B$. afzelii, $I_{B b}$ is the total number of nymphs infected with B. burgdorferi s.l. as determined by qPCR, and $N_{t}$ is the total number of nymphs tested in a plot. We assumed that all genospecies had an equal probability of being sequenced successfully.

We used the calculated infection prevalence with $B$. afzelii and the infection prevalence as determined by GPCR for $B$. miyamotoi and $\mathrm{Ca}$. N. mikurensis to estimate the density of questing nymphs infected by these pathogens as, 


$$
D I N_{P i}=N I P_{P i} \cdot D O N
$$

Equation 6.3

where $D I N_{P i}$ is the density of questing nymphs infected with pathogen species $i\left(100 \mathrm{~m}^{-2}\right), N I P_{P i}$ is the infection prevalence in questing nymphs with pathogen species $i$, and $D O N$ is the density of questing nymphs as determined by blanketdragging $\left(100 \mathrm{~m}^{-2}\right)$.

\section{Statistical analysis}

Statistical analyses were performed in R 3.2.3 ${ }^{81}$ using the nlme package ${ }^{236}$ to perform linear mixed models (LMMs) and the g/mmADMB package ${ }^{201}$ to perform generalized linear mixed models (GLMMs). We first tested if temperature and humidity influenced our estimates of nymphal density ${ }^{208}$, which was not the case (methods and results in Appendix S6.2).

We used confirmatory path analysis using directional separation, to simultaneously test for all predicted relationships (Figure 6.1) ${ }^{237}$. Confirmatory path analysis was used to quantify indirect correlations based on only observational data. When this type of data has a hierarchical or multilevel structure, directional separation can be used to test causal models. We determined and tested the independence claims for the causal model in Figure 6.1 and calculated $C$ values as described by Shipley ${ }^{237}$ for each of the three pathogens separately. Causal models were rejected if the $C$ value was unlikely to have occurred by chance $(p<0.05)$. We used estimates on plot level for all parameters to be able to test the causal model.

To determine which parameter estimate for tick burden we could best use, we tested for a correlation between larval burden and nymphal prevalence per plot. As the two were positively correlated (binomial GLMM with logit link function, $\beta=2.21, p<0.001$ ), we only included larval burden in the path analyses. We tested for a correlation of nymphal prevalence with deer and predator abundance, and rodent density using a GLMM with a binomial distribution, a logit link function, and a random intercept per vegetation type nested within year.

For each pathogen, we determined the independence claims for the causal model (Table S6.1), and tested these using an LMM and GLMMs with a negative binomial distribution and log link function (table S6.1). We tested the likelihood 


\section{6 | Chapter 6}

of all three causal models by testing the $C$ statistic with a chi-square test ${ }^{237}$. Causal models that were not rejected based on their $C$ statistic $(p>0.05)$ were further tested using an LMM and GLMMs to determine path coefficients. The correlation of rodent density with predator abundance was tested using an LMM. The correlation of median larval burden on rodents with predator and deer abundance, and rodent density was tested using a GLMM with a negative binomial distribution and a log link function, and the correlation of DIN with rodent density and median larval burden was tested using a GLMM with a negative binomial distribution and a log link function, for each pathogen separately. All mixed models were allowed to have a random intercept per vegetation type nested within year.

We did an additional analysis to test for a possible correlation of median larval burden on rodents with the relative abundance of another important host species, European hare (Lepus europaeus) ${ }^{238}$. The extended model for median larval burden on rodents included deer, predator, and hare abundance, and rodent density as parameters using a GLMM with a negative binomial distribution, a log link function, and a random intercept per vegetation type nested within year, which was compared to the simpler model used in the path analysis using AICc values ${ }^{82}$.

To approximate a normal distributions for all parameters not transformed by a GLMM, we used a $\log _{10}$ transformation. We added a value of 0.01 , the minimal passage rate found for deer, to the raw deer passage rate to avoid transformation problems due to three areas with no records of deer. After transformation, we standardized all parameters by extracting the mean, and dividing by two standard deviations ${ }^{223}$ to obtain standardized regression coefficients.

We used a chi-square test to test for differences in observed prevalence of co-infections with the different pathogens in all questing nymphs, and expected prevalence of co-infections based on the infection prevalence of the separate pathogens, to test for associations between the pathogens.

\section{Results}

We recorded 14 mammal species with camera trapping (Table S5.1). The median passage rate per plot was $0.04 \mathrm{~m}^{-1} \mathrm{~d}^{-1}$ for deer (range: $0-0.84$ ) and $0.01 \mathrm{~m}^{-1} \mathrm{~d}^{-1}$ for predators (range: $0.001-0.033$; Table S6.2). We caught a total of 626 forest 


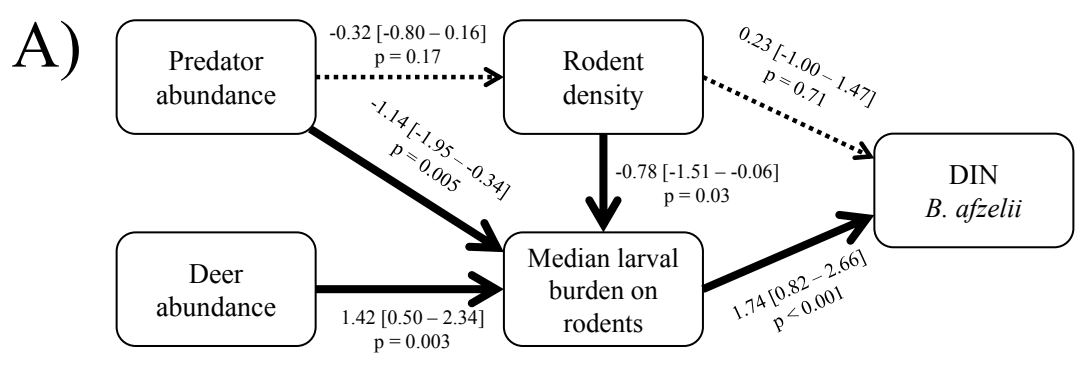

B)
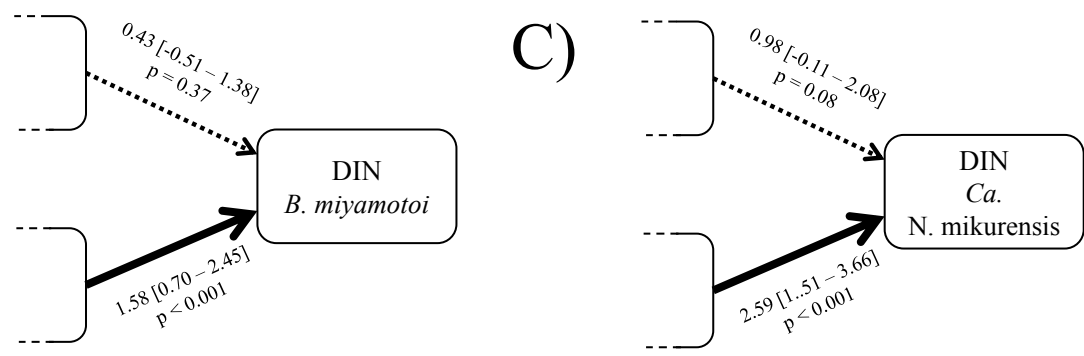

Figure 6.2. Path diagrams showing direct and indirect correlations of vertebrate assemblage composition with median larval burden on rodents and density of infected nymphs (DIN) for A) Borrelia afzelii, B) B. miyamotoi and C) Candidatus Neoehrlichia mikurensis. The left part of the diagram is identical for all three models and therefore only shown once. Thick lines indicate statistically supported paths. Path coefficients have $95 \%$ confidence interval between brackets.

rodents of three species: Bank vole, Field vole and Wood mouse. These rodents carried a total of 10,576 larvae and 158 nymphs of I. ricinus. The median rodent density per plot was 24 ha $^{-1}$ (range: 0 - 327.7), median larval burden per rodent per plot was 11 larvae (range: 1-52), and average nymphal prevalence per plot was 0.20 of rodents infested (range: $0-0.71$ ) between plots (Table S6.2). We could not determine tick burden on rodents in one site (Duin en Kruidberg), where we caught zero rodents, hence excluded this plot from the analysis.

We caught a total of 16,617 nymphs by blanket-dragging in all twenty plots, of which 14,025 were analysed for the presence of B. afzelii, B. miyamotoi, and Ca. N. mikurensis. The median density of infected nymphs (DIN) was $2.5100 \mathrm{~m}^{-2}$ (range: 0 - 22.2) for $B$. afzelii, $1.2100 \mathrm{~m}^{-2}$ (range: 0 - 5.9) for $B$. miyamotoi and $2.6100 \mathrm{~m}^{-2}$ (range: 0 - 24.5) for Ca. N. mikurensis (Table S6.2).

Among questing nymphs we found an overrepresentation of co-infection of $B$. afzelii and B. miyamotoi (observed $\%=0.08$, expected $\%=0.05, x^{2}$-value $=4.3$, $p=0.04$ ), co-infection with B. afzelii and Ca. N. mikurensis (observed $\%=0.54$, expected $\%=0.13, X^{2}$-value $\left.=168.1, p<0.001\right)$, co-infection with $B$. miyamotoi 


\section{8 | Chapter 6}

and Ca. N. mikurensis (observed $\%=0.18$, expected $\%=0.12, X^{2}$-value $=4.0, p$ $=0.047$ ), and co-infection of all three pathogens (observed $\%=0.02$, expected $\%=0.003, x^{2}$-value $=19.8, p<0.001$ )

The tested path models were supported for all three pathogens $(B$. afzelii: $\mathrm{C}=$ 3.25, $p=0.78$; B. miyamotoi: $\mathrm{C}=2.74, \mathrm{p}=0.84$; $\mathrm{Ca}$. N. mikurensis: $\mathrm{C}=1.84, \mathrm{p}$ $=0.93)$. However, not all path coefficients were significantly different from zero (Figure 6.2): There was no correlation between predator passage rate and rodent density (LMM: $\beta=-0.32, p=0.17$ ), and no correlation between rodent density and DIN for any of the three pathogens ( $B$. afzelii GLMM: $\beta=0.23, p=0.71$; B. miyamotoi GLMM: $\beta=0.43, p=0.37 ; \mathrm{Ca}$. N. mikurensis GLMM: $\beta=0.98, p$ $=0.08$; Figure 6.2). Larval burden on rodents increased with deer abundance (GLMM: $\beta=1.42, p=0.003$ ), and decreased with predator abundance (GLMM: $\beta$ $=-1.14, p=0.005)$ and rodent density (GLMM: $\beta=-0.78, p=0.03)$, as predicted (Figure 6.2). Nymphal prevalence on rodents decreased with predator abundance (GLMM: $\beta=-0.66, p=0.02$ ) and rodent density (GLMM: $\beta=-2.09, p<0.001$ ), as predicted, but was not correlated with deer abundance (GLMM: $\beta=0.64, p$ $=0.10$ ). For each of the three pathogens, DIN increased with larval burden on rodents, as predicted ( $B$. afzelii GLMM: $\beta=1.74, p<0.001$; $B$. miyamotoi GLMM: $\beta=1.58, p<0.001$; Ca. N. mikurensis GLMM: $\beta=2.59, p<0.001$; Figure 6.3).

The results for the extended model for median larval burden (including hare abundance) were similar to the results from the simpler model in the path analysis. Larval burden on rodents increased with deer abundance (GLMM: $\beta=$ 1.31, $p=0.003$ ), and decreased with predator abundance (GLMM: $\beta=-1.11, p$ $=0.007$ ) and rodent density (GLMM: $\beta=-0.82, p=0.03$ ). Median larval burden on rodents was not correlated with European hare abundance (GLMM: $\beta=-0.17$, $p=0.66)$. The extended model had a lower model fit than the simpler model in the path analysis $(\triangle \mathrm{AICC}=6.07)$.

\section{Discussion}

Interactions between vertebrate hosts are hypothesized to influence the density of infected nymphs (DIN), which is an often used ecological parameter to determine tick-borne disease risk ${ }^{39}$. Therefore, changes in fauna composition may have cascading effects on tick-borne disease risk. We used path analysis to investigate three different mechanisms by which different hosts can influence DIN: 1) encounter reduction between ticks and reservoir hosts, 2) vector augmentation 

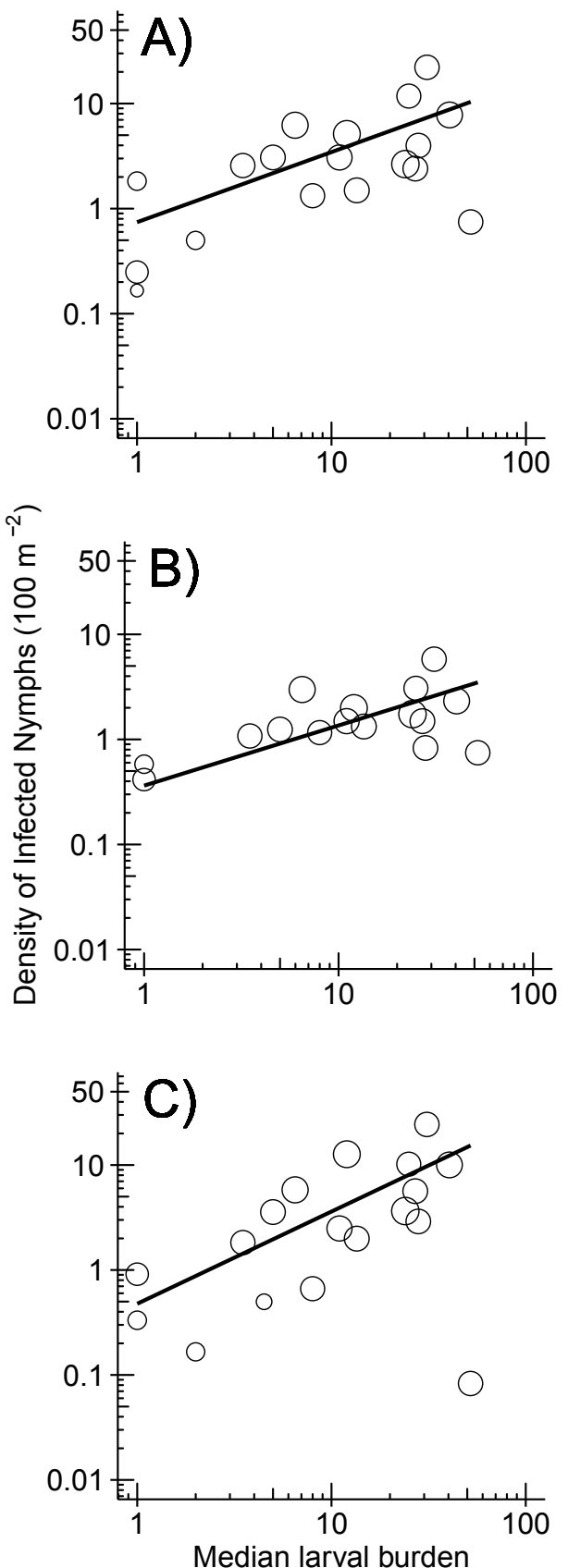

Figure 6.3. Correlation between median larval burden on rodents and density of infected nymphs (DIN) for A) Borrelia afzelii, B) B. miyamotoi and C) Candidatus Neoehrlichia mikurensis. Symbol size varies with sample size. 


\section{0 | Chapter 6}

and 3) reservoir host regulation ${ }^{31}$. We found that variation in the density of $I$. ricinus nymphs infected with three rodent-transmitted tick-borne pathogens was explained by differences in vertebrate assemblage composition. The patterns found are consistent with vector augmentation by deer, and encounter reduction by rodents and predators, but not with reservoir host regulation by predators. This means that changes in fauna composition may have cascading effects on rodent-transmitted tick-borne disease risk.

The support for augmentation of $I$. ricinus larvae by deer is consistent with a previous study in which larval burden on rodents increased with deer density 32 . This relationship is straightforward as more deer can feed more adult ticks, resulting in a larger number of larvae in the environment ${ }^{32,38}$ (Chapter 4 ), and was reflected in our study by a higher larval burden on rodents in areas with high deer abundance (Figure 6.2). Our results are also consistent with previous studies that hypothesized that deer could indirectly increase disease risk by increasing larval densities and subsequently the number of larvae feeding on reservoircompetent host species ${ }^{208,239}$. Some studies have suggested a possible dilution effect of high deer densities on disease risk, where deer, which are incompetent as hosts for several pathogens (e.g. B. burgdorferi s.l. ${ }^{240}$ ), attract (infected) nymphs that would otherwise feed on reservoir competent host species ${ }^{32}$. However, we did not find support for this hypothesis, as deer abundance was not correlated with nymphal prevalence and there was a strong positive correlation with larval burden on rodents. The removal of deer from a system would thus lower disease risk, by reducing the number of larvae in the environment and thereby reducing the encounter rate between reservoir hosts and larvae .

The DIN for rodent-transmitted tick-borne pathogens decreased with rodent density, apparently due to a negative relationship between rodent density and larval burden and nymphal prevalence on rodents (encounter reduction). However, in contrast to previous studies (e.g., Ostfeld et al. ${ }^{204}$ ), we did not find a direct correlation between rodent density and DIN. This could be due to the fact that we measured rodent density and DIN in the same year, while rodent population densities fluctuate between years ${ }^{204}$ and $I$. ricinus has a multiyear life cycle, in which the larvae feeding on rodents in one year will quest as nymphs in the vegetation in the following year ${ }^{8}$. A negative correlation between larval burden on rodents and rodent density was previously found both in North America $^{241}$ and in Europe ${ }^{95}$. There are two possible mechanisms for this negative relationship. First, an increase in rodents could result in a diluted distribution of 
the total number of immature ticks over more rodents, resulting in lower larval burden and nymphal prevalence. Secondly, the day-range of rodents was found to decrease with increasing rodent density ${ }^{213,242}$. This could result in a lower rate of rodent-tick encounter, resulting in lower larval burden and lower nymphal prevalence on rodents, as found in our study. As these two mechanisms are not mutually exclusive, studies investigating the effect of rodent density on spatial behaviour of rodents in relation to questing ticks are needed.

Ostfeld and Holt ${ }^{160}$ theorised that generalist predators can influence disease risk by reducing reservoir host densities (reservoir host regulation). We did not find a negative correlation between predator abundance and rodent density. This could be due to the fact that two mechanisms function at the same time: 1) high predator abundance reduces rodent population densities (negative correlation) ${ }^{243}$, while at the same time, 2) patches with high rodent density attract predators from the surrounding area (positive correlation) ${ }^{244}$. Therefore, correlational studies on a small spatial scale ( $\leq 1$ ha) might not be able to show reservoir host regulation. Besides the presence of predators, food supply could be an important determinant of rodent densities in our plots ${ }^{245}$, masking possible effects of predators on their prey population.

We did find an indirect negative correlation between the relative abundance of predators and DIN, via larval burden on rodents (encounter reduction). Furthermore, we found that nymphal prevalence on rodents decreased with predator abundance. These correlations could be due to several mechanisms. First, rodents with very high tick burdens could be more likely to be predated on, which would result in a more uniform distribution of ticks over rodents in sites with high predator abundance, resulting in lower median burdens. Second, addition of predators might result in a larger part of the larvae feeding on predators, reducing rodent tick burden. We consider this unlikely, as predators in general have very low larval burdens ${ }^{190,246}$ (Chapter 2). Therefore, we argue that the most likely mechanism involves a change in the spatial behaviour of rodents as a response to predation pressure ${ }^{214}$ (Chapter 5), resulting in a lower encounter rate between ticks and individual rodents. Previous studies have shown that rodents can change both their activity pattern and spatial habitat use as a response to predators or the presence of predator scent ${ }^{28,229}$. The negative correlation that we found may be caused by a decrease of rodent activity in response to the relative abundance predators. This indirect correlation may also explain the negative correlation found between Red fox density and Lyme borreliosis cases 


\section{2 | Chapter 6}

in a large-scale study in North America ${ }^{247}$.

In North America, competition for questing ticks between different host species with varying reservoir competence has been put forward as the main mechanism by which vertebrate assemblage structure influences DIN for B. burgdorferi s. . $^{20,31,241}$. In Europe, however, the majority of I. ricinus larvae feed on small rodents, and other species - such as European hares - might not contribute much to feeding larval I. ricinus due to their low densities ${ }^{64,190}$ (Chapter 2). This was supported by our analysis, as we found no correlation between the relative abundance of European hares and I. ricinus burden on rodents. Therefore, there is no evidence for European hares luring away larvae from rodents, one of the suggested mechanisms for encounter reduction ${ }^{31}$. Furthermore, all correlations between tick burden on rodents and vertebrate assemblage parameters in our study could be explained by behaviour of rodents (Chapter 5). It has been suggested that the spatial behaviour of individual hosts is more important in determining tick burden than the availability of ticks ${ }^{158}$. This suggests that, at least in Europe, a change in spatial behaviour due to the presence of conspecifics, competitors or predators is a more plausible explanation for the difference in tick burden on rodents between sites with different vertebrate assemblages than competition for ticks between the different host species.

Our study is among the first to use camera traps to study the interaction between vertebrate communities and prevalence of disease. We show that the relative abundance of medium-sized to large mammals quantified with camera traps can be used to study mechanisms underlying disease dynamics at a small spatial scale. Prior studies investigated the role of vertebrate hosts on tick-borne disease dynamics on large spatial scales (e.g., Levi et al. ${ }^{247}$ ), probably because abundance data from hunting-bag statistics and species distributions are available on this scale ${ }^{248}$. The interactions between hosts, ticks and tickborne pathogens, however, occur on much smaller spatial scales, such as the 1 ha-forest patches that we investigated. We draw attention to the issue of scale when studying predictive patterns in disease ecology. Camera traps may become very important for collecting data on vertebrate assemblages at smaller spatial scales, provided that biases due to factors other than animal density or activity are accounted for ${ }^{167,198}$ (Chapter 3).

Concluding, we found support for vector augmentation by deer and encounter reduction by conspecifics and predators. We did not find support for reservoir host regulation by predators, nor a correlation between reservoir host density 
and tick-borne disease risk as measured by the DIN. We found further support for a reservoir role of forest-dwelling rodents for $\mathrm{B}$. miyamotoi and $\mathrm{Ca}$. N. mikurensis as co-infections of both pathogens with $B$. afzelii in nymphs were found more than expected by chance. This study is, to our knowledge, the first to find empirical support for changes in tick-borne disease risk resulting from behavioural responses of hosts to changes in vertebrate assemblage composition. The emergence of a cascading effect of predator abundance on tick-borne disease risk calls for the conservation of medium-sized predators like Polecat, Pine marten and Red fox, of which especially the latter is hunted in many countries in Europe ${ }^{249}$. 


\section{4 | Chapter 6}

\section{Appendix to Chapter 6}

Appendix S6.1. Primers and PCR program used for detection of Borrelia burgdorferi s.l. and B. miyamotoi.

For the detection of B. burgdorferi s.I. and B. miyamotoi, a triplex qPCR, targeting ospA and flaB genes, was used. The sequences of primers and probes are given in Table S6.1.1.

Table S6.1.1. Primers and probes used in the PCR analyses

\begin{tabular}{|c|c|c|}
\hline $\begin{array}{l}\text { Target gene/primer \& } \\
\text { probe }\end{array}$ & $\begin{array}{l}\text { Amplicon } \\
\text { length }\end{array}$ & Sequence \\
\hline $\begin{array}{l}\text { OspA (Outer mem- } \\
\text { brane Protein A, } \\
\text { B. burgdorferi s.l.) }\end{array}$ & $\pm 139 \mathrm{bp}$ & \\
\hline B-OspA_modF & & 5'-AAT ATT TAT TGG GAA TAG GTC TAA-3' \\
\hline B-OspA_borAS & & 5'-CTT TGT CTT TTT CTT TRC TTA CA-3' \\
\hline B-OspAmodPatto & & 5'-Atto520-AAG CAA AAT GTT AGC AGC CTT GA-BHQ1-3' \\
\hline $\begin{array}{l}\text { FlaB (Flagelin B, } \\
\text { B. burgdorferi s.I.) }\end{array}$ & $\pm 89 \mathrm{bp}$ & \\
\hline B-FlaB-F & & 5'-CAG AIA GAG GTT CTA TAC AIA TTG AIA TAG A-3' \\
\hline B-FlaB-Rc & & 5'-GTG CAT TTG GTT AIA TTG CGC-3' \\
\hline B-FlaB-Rt & & 5'-GTG CAT TTG GTT AIA TTG TGC-3' \\
\hline B-FlaB-Patto & & 5'-Atto425-CAA CTI ACA GAI GAA AXT AAI AGA ATT GCT GAI CA-Pho-3' \\
\hline $\begin{array}{l}\text { FlaB (Flagelin B, } \\
\text { B. miyamotoi) }\end{array}$ & $\pm 155 \mathrm{bp}$ & \\
\hline FlabBm.motoiF2 & & 5'-AGA AGG TGC TCA AGC AG-3' \\
\hline FlabB.m.motoiR3 & & 5'-TCG ATC TTT GAA AGT GAC ATA T-3' \\
\hline FlabBm.motoiPro & & 5'-ATT0647N-AGC ACA ACA GGA GGG AGT TCA AGC-BHQ2-3' \\
\hline
\end{tabular}

$\mathrm{X}=\mathrm{BHQ}-1-\mathrm{dT} ; \mathrm{BHQ}=\mathrm{Black}$ Hole Quencher

The qPCR was performed using the iQ Multiplex Powermix PCR reagent kit (BioRad Laboratories, Hercules, USA), in a LightCycler 480 Real-Time PCR System (F. Hoffmann-La Roche, Basel, Switzerland). The reaction mix consisted of iQ multiplex Powermix, $100 \mathrm{nM}$ of the B-FlaB-Rc and B-FlaB-Rt primers, $200 \mathrm{nM}$ 
of the B-FlaB-F, FlabBm.motoiF2, and FlabB.m.motoiR3 primers, $400 \mathrm{nM}$ of the B-OspA_modF and B-OspA_borAS primers, $100 \mathrm{nM}$ of the B-OspAmodPatto probe, $200 \mathrm{nM}$ of the B-FlaB-Patto and FlabBm.motoiPro probes, and $3 \mu \mathrm{l}$ of template DNA in a final volume of $20 \mathrm{ul}$. Cycling conditions included an initial activation of the iTaq DNA polymerase at $95^{\circ} \mathrm{C}$ for $5 \mathrm{~min}$, followed by 60 cycles of a $5 \mathrm{~s}$ denaturation at $95^{\circ} \mathrm{C}$, followed by a $35 \mathrm{~s}$ annealing-extension step at $60^{\circ} \mathrm{C}$ (ramp rate $2.2^{\circ} \mathrm{C} / \mathrm{s}$ and a single point measurement at $60^{\circ} \mathrm{C}$ ) and a cooling cycle of $37^{\circ} \mathrm{C}$ for $20 \mathrm{~s}$. Analysis was performed using the second derivative calculations for crossing point values. For each run positive and negative controls and blank samples were included.

Appendix S6.2. Methods and results for analysis of influence of temperature and humidity on estimates of nymphal density.

\section{Methods}

Air temperature and humidity can influence the efficiency of drag sampling, resulting in biased tick density estimates ${ }^{208}$. We therefore measured air temperature and relative humidity at the start and the end of each sampling session within the vegetation using a hygro-thermometer (TH-1; Amprobe, Everett, WA, U.S.A.) and averaged these values per drag session. These were correlated to nymphal densities using a generalized linear mixed model with a negative binomial distribution, log link function and a random intercept per plot nested within year, as implemented in the g/mmADMB package in $\mathrm{R}^{81,201}$. We used a random intercept per plot per year to account for multiple measurements in each plot. Both temperature and humidity estimates were standardized by extracting the mean and dividing by 2 standard deviations in order to obtain standardized correlation coefficients ${ }^{223}$.

\section{Results}

Nymphal density per sampling session was not correlated to either temperature (GLMM: $\beta=-0.15, p=0.23$ ) or relative humidity (GLMM: $\beta=-0.20, p=0.09$ ). We therefore did not correct for these variables in our path analysis. 


\section{6 | Chapter 6}

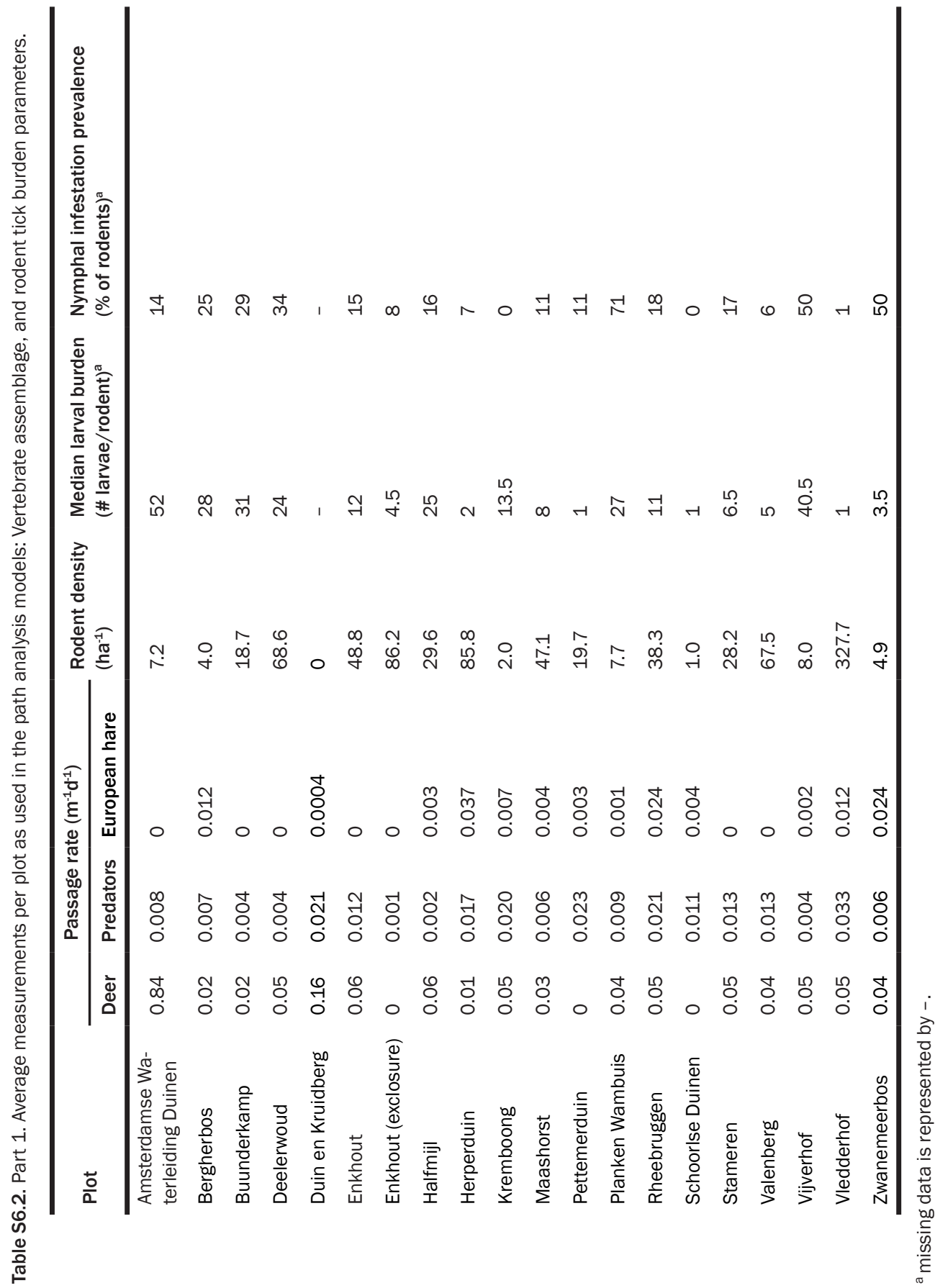




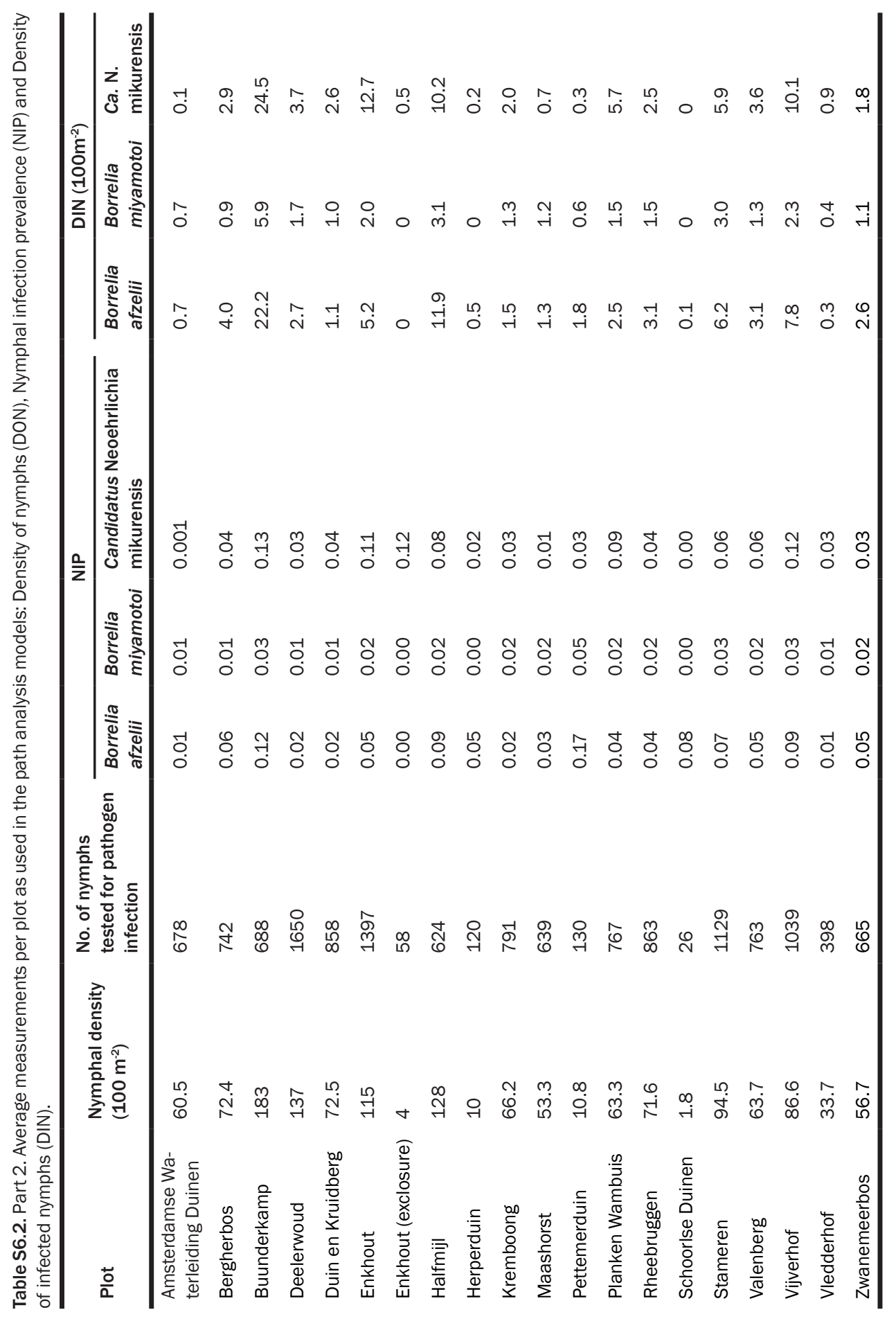


To be or not to be competent

that is the pathogen's question

is it or is it not

competent

is that the question?

\section{Ronald Hofmeester}




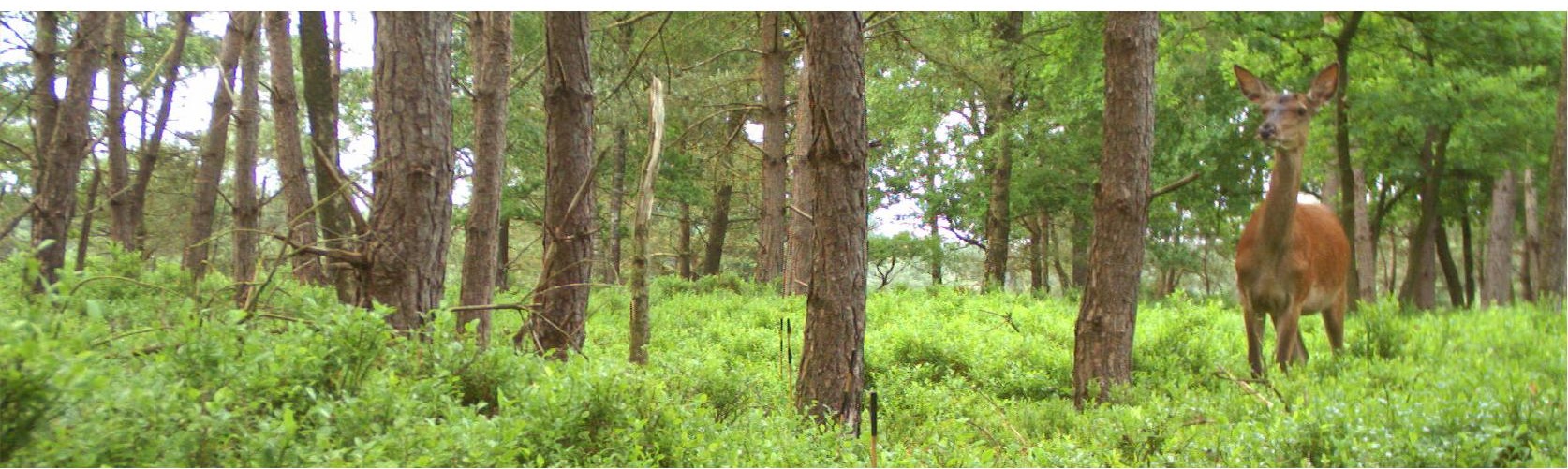

T.R. Hofmeester, E.C. Coipan, M. Fonville, P.A. Jansen, H.H.T. Prins, H. Sprong \& S.E. van Wieren

\section{No Support for a Dilution Effect of Host Species Richness on TICK-BORne Disease RISK IN EUROPE}

The prevalence of pathogens is predicted to increase with the relative abundance of reservoir-competent hosts (those that transmit the pathogen) - which is assumed to decrease with species richness - and to decrease with the relative abundance of reservoir-incompetent hosts (those that do not transmit the pathogen) - which is assumed to increase with species richness - but this has hardly been tested empirically. We investigated the relationship between pathogen prevalence in questing Ixodes ricinus nymphs, densities of infected nymphs, the abundance of reservoir-competent and reservoir-incompetent hosts, and host species richness for seven tick-borne pathogen species in twenty 1-ha forest plots in the Netherlands, with a wide range in host abundance and host species richness as measured with camera traps and live traps. We found that infection prevalence in nymphs for six pathogen species, and density of infected nymphs for three pathogen species increased with abundance of reservoir-competent hosts, and that the abundance of several reservoir-competent host species rose with host species richness. Nymphal infection prevalence decreased with reservoir-incompetent host abundance for three pathogens. To test if correlations found on this small spatial scale resulted in large-scale differences, we tested for a correlation of mammalian host species richness with Lyme borreliosis incidence for 17 countries in Western Europe. At this scale, Lyme borreliosis incidence increased with mammal richness as well. These results are in disagreement with a dilution effect of species richness for tick-borne pathogens in Europe. Rather, the relative densities of reservoircompetent and reservoir-incompetent host species are the most important parameters determining tick-borne disease risk. 


\section{0 | Chapter 7}

\section{Introduction}

Vector-borne pathogens often use multiple host species to complete their enzootic cycle. If host species differ in their reservoir competence (i.e., their ability to transmit the pathogen to feeding vectors), pathogen prevalence in vectors can vary between populations with different host assemblages ${ }^{34,190,250}$ (Chapter 2). The distribution of vectors over the different host species dependent on the densities of the different host species will thus determine the prevalence of pathogens in the vectors ${ }^{21}$.

Theoretical studies have predicted that the prevalence of a pathogen in vectors should increase with the abundance of reservoir-competent host species, because a larger proportion of vectors will feed on these species (amplification effect), while pathogen prevalence in the vectors should decrease with the abundance of reservoir-incompetent host species, because a larger proportion of vectors will then feed on these species (dilution effect) ${ }^{27,33}$. Furthermore, Van Buskirk and Ostfeld ${ }^{27}$ predicted that the density of reservoir-competent hosts is the main determinant of the density of infected vectors, a widely used measure of disease risk ${ }^{27}$. When the host species with the highest reservoir competence occurs in many different communities and reaches the highest abundance compared to other species in species-poor communities, both the prevalence of vector-borne pathogens and the density of infected vectors should decrease with host diversity: a dilution effect of host species richness ${ }^{3}$.

For tick-borne pathogens vectored by a generalist species, the main conditions for a dilution effect to occur are that 1) pathogen prevalence increases with the density of host species with the highest reservoir competence and that 2) the density of these host species decreases with host species richness ${ }^{3}$. It has been shown that a dilution effect of host species richness occurs in many disease systems ${ }^{251}$, including the Lyme borreliosis system in North America, where a dilution effect of host species richness was found both at a small ${ }^{230}$ and a large spatial scale ${ }^{248}$. However, the mechanisms underlying any negative correlation with host species richness remain relatively unstudied ${ }^{218}$.

In Europe, the Sheep tick, Ixodes ricinus, is the most important vector for vector-borne diseases such as Lyme borreliosis and tick-borne encephalitis ${ }^{57}$. Although $I$. ricinus parasitizes more than 100 host species, the majority of $I$. ricinus individuals feed on a relatively small selection of host species: larvae mostly on small mammals, nymphs on thrushes, and most adults on deer ${ }^{190}$. 
Species within these host groups happen to also be the most important reservoirs for tick-borne pathogens in Europe. Small mammals such as the Bank vole (Myodes glareolus) and Wood mouse (Apodemus sylvaticus) are important hosts for Borrelia afzelii, B. bavariensis, B. miyamotoi, B. spielmanii, and Candidatus Neoehrlichia mikurensis ${ }^{50,190,252}$ (Chapter 2). Eurasian blackbird (Turdus merula) and Song thrush (T. philomelos) are important reservoir hosts for B. garinii and B. valaisiana ${ }^{190,252}$ (Chapter 2), and Roe deer (Capreolus capreolus), Red deer (Cervus elaphus) and Fallow deer (Dama dama) are important reservoir hosts for Anaplasma phagocytophilum ${ }^{42,51,154}$. Which host species are most important for the transmission of B. burgdorferi s.s. in Europe is not well known, and Eurasian red squirrel (Sciurus vulgaris), small mammals and birds have all been suggested as reservoir hosts for this genospecies ${ }^{48,162}$. Because roles of host species differ between pathogen species, there is substantial debate if the conditions for a dilution effect for tick-borne pathogens in Europe are met $^{158}$. It has been proposed that tick-borne disease risk in Europe as measured by the density of infected nymphs might actually increase with vertebrate species richness ${ }^{253}$.

We studied the correlation of tick-borne disease risk with host species richness in Europe at two spatial scales. First, we simultaneously studied the relationship of seven tick-borne pathogen species that are all transmitted by I. ricinus, but are maintained by different host species, and therefore differ in their hypothesized prevalence in vertebrate assemblages (Table 7.1). Specifically, for each of these pathogens, we studied the nymphal infection prevalence (NIP) and density of infected nymphs (DIN) in twenty 1-ha forest plots scattered across the Netherlands that differed in their vertebrate assemblage. Second, we investigated whether Lyme borreliosis incidence in 17 Western European countries ${ }^{56}$ was correlated with species richness of mammalian hosts. We expected that 1) NIP and DIN for all pathogens would increase with the abundance of reservoir-competent hosts (Table 7.1), and 2) would decrease with the abundance of reservoir-incompetent hosts. Next, we expected that 3 ) the abundance of reservoir-competent hosts would increase with host species richness, as multiple host species have a high realized reservoir competence for each of the pathogens, which should result in 4) a positive correlation of Lyme borreliosis incidence with species richness of mammalian hosts both at a small and large spatial scale. 


\section{2 | Chapter 7}

\section{Methods}

We compared pathogen prevalence between twenty study plots of 1 ha in forested sites in the Netherlands (Table S4.1) that widely ranged in host species presence and abundance. Sites were at least $10 \mathrm{~km}$ apart (or $5 \mathrm{~km}$ apart but separated by a busy motorway) to ensure separate host populations. We sampled eleven plots in 2013 and in nine in 2014. In one site, Enkhout, we sampled in two separate plots of which one was within a fenced forest patch (3 ha) which had been fenced three years prior to our sampling.

\section{Reservoir host abundance}

We based our expectations for the correlation between the presence and abundance of host species and infection prevalence of tick-borne pathogens on the assumption that questing ticks are more likely to encounter a host species as the latter is present more often. This could be either due to a high host density

Table 7.1. Pathogens and main reservoir-competent host species for which a positive correlation is expected with nymphal infection prevalence and density of infected nymphs. Taxonomic groups are printed in bold.

Pathogen
Small mammal-transmitted
Borrelia burgdorferi genospecies
(B. afzelii and B. spielmanii)

Bird-transmitted

Borrelia burgdorferi genospecies (B. garinii and B. valaisiana)

Borrelia burgdorferi s.s.

Candidatus

Neoehrlichia mikurensis

Anaplasma phagocytophilum

Borrelia miyamotoi
Main reservoir host species

References

Wood mouse (Apodemus sylvaticus), Bank vole (Myodes glareolus), Field vole (Microtus agrestis), and Common 94,96 shrew (Sorex araneus) (Small mammals)

Black bird (Turdus merula) and Song thrush ( $T$. ${ }_{125,254}$ philomelos) (Birds)

Eurasian red squirrel (Sciurus vulgaris), Wood mouse, Bank vole (Small mammals), and Birds

Bank vole and Wood mouse (Rodents)

233,255

Roe deer (Capreolus capreolus), Red deer (Cervus 42,51 elaphus), and Fallow deer (Dama dama) (Ungulates)

Bank vole, Wood mouse (Rodents) and trans-ovarial transmission 
or a high activity level of host individuals. Therefore, we used the number of animals walking past a location in the plot per day, measured as their passage rate, as a measure of host availability to ticks.

We used camera traps (HC500, RECONYX Inc., Holmen, WI, U.S.A.) to determine the passage rate of mammals and birds in each forest plot as the rate of detection by camera traps during March - November, the main activity period of Ixodes ricinus in the Netherlands ${ }^{156}$. In each plot we used the protocol described in Chapter 3 to obtain eighteen camera positions, resulting in approximately 500 camera trap days per plot (Table S4.1). All passages of one animal or group of animals were stored as a separate sequence, and annotated using a custommade photo-processing tool called 'Agouti' (cf. Kays et al. ${ }^{178}$ ).

For every sequence of photographs, we determined the species and number of animals that crossed the midline of the view. For each crossing, we determined the distance from the camera with the aid of markers placed at $2.5 \mathrm{~m}$ intervals in front of the camera ${ }^{198}$ (Chapter 3). Following the methods described in Chapter 3, we determined the effective detection distance (EDD) of all mammal species per habitat type using a point model with a detection probability described by a half-normal function with $\log _{10}$-transformed body mass as covariate. As we did not have enough detections per habitat type for birds, we estimated EDD for all habitat types simultaneously, using a hazard-rate function with $\log _{10}$-transformed body mass as covariate. The EDD estimates for mammals are given in Table S5.1 and those for birds are given in Table S7.1. These estimates were used to determine passage rates per species per camera location as,

$$
P R_{i}=\frac{x_{i}}{t \cdot E D D_{i_{h}}}
$$

Equation 7.1

where $P R_{i}$ is the passage rate of species $i\left(\right.$ meter $^{1}$ day $\left.^{-1}\right), x_{i}$ the number of passages of species $i, t$ the total time at which the camera was active (in days), and $E D D_{\text {in }}$ the effective detection distance of species $i$ in habitat type $h$ (in meters). We used the arithmetic mean to average this rate over all camera locations to determine the passage rate per species per plot.

To obtain passage rate estimates for groups of species with a high reservoir competence, we simply summed passage rates of host species. Deer included Roe deer, Red deer and Fallow deer, and ungulates included all deer and Wild boar (Sus scrofa), another possible reservoir-competent species $A$. 


\section{4 | Chapter 7}

phagocytophilum ${ }^{51}$. Common thrushes included Blackbird and Song thrush, and birds included both species of thrush, Blue tit (Cyanistes caeruleus), Chaffinch (Fringilla coelebs), Great tit (Parus major), Mistle thrush (Turdus viscivorus) and Redwing (Turdus iliacus), i.e., all observed species that were reservoir-competent for bird-transmitted B. burgdorferi s.l. genospecies ${ }^{190}$ (Chapter 2). We determined the passage rate for reservoir-incompetent species by summing - per pathogen species - the passage rates of all species not known to transmit the pathogen in previously published studies ${ }^{42,51,190}$ (Chapter 2 ). For clarity we will refer to passage rates as "abundance", but strictly speaking it is a combination of density and activity in a plot.

Small-mammal densities were estimated by live trapping as described in Chapter 5. We estimated densities of Bank vole, Field vole (Microtus agrestis) and Wood mouse using capture-mark-recapture models for closed populations implemented in MARK $^{222}$ assuming that the probability of capture and the probability of recapture were equal and constant over trapping sessions. For plots in which we caught too few rodents to estimate densities using MARK and for the density estimates for Common shrew (Sorex araneus) and Pigmy shrew (Sorex minutus) we used the 'minimum number of animals known alive' as the density estimate. We combined the densities of Bank vole, Wood mouse and Field vole to estimate rodent density and combined the densities for all five small mammal species to estimate small-mammal density per plot.

Table 7.2. Observed and expected co-infections of pathogens in questing nymphs (observed\% / expected\%).

\begin{tabular}{|c|c|c|c|c|c|}
\hline & $\begin{array}{l}\text { Bird-transmitted } \\
\text { Borrelia spp. }^{a}\end{array}$ & $\begin{array}{l}\text { Borrelia } \\
\text { burgdorferi } \\
\text { s.s. }\end{array}$ & $\begin{array}{l}\text { Anaplasma } \\
\text { phagocytophilum }^{a}\end{array}$ & $\begin{array}{l}\text { Borrelia } \\
\text { miyamotoi }^{\mathrm{a}}\end{array}$ & $\begin{array}{l}\text { Candidatus } \\
\text { Neoehrlichia } \\
\text { mikurensis }^{a}\end{array}$ \\
\hline B. afzelii & $0 / 0.02$ & 0 / 0.003 & $0.03 / 0.07$ & $\begin{array}{l}0.08 / \\
0.04 *\end{array}$ & $\begin{array}{l}0.51 / \\
0.12 * * *\end{array}$ \\
\hline $\begin{array}{l}\text { Bird-transmitted } \\
\text { Borrelia spp. }\end{array}$ & & $0 / 0.001$ & $0 / 0.03 *$ & $0.01 / 0.02$ & $\begin{array}{l}0.01 / \\
0.06 * *\end{array}$ \\
\hline B. burgdorferi s.s. & & & $0 / 0.004$ & $\begin{array}{l}0.01 / \\
0.003\end{array}$ & 0 / 0.01 \\
\hline A. phagocytophilum & & & & 0.05 / 0.07 & $\begin{array}{l}0.04 / \\
0.19 * * *\end{array}$ \\
\hline B. miyamotoi & & & & & 0.17 / 0.12 \\
\hline
\end{tabular}

a Associations that differed significantly from expected prevalence are printed in bold, with * $p<0.05, * * p<$ $0.01, * * * p<0.001$, based on 14,044 tested nymphs. 
Only species known to host I. ricinus ${ }^{145,190}$ (Chapter 2) were taken into account in our analyses. Assumed reservoir competence and reservoir incompetence for all host species used in the analyses are given in Table S7.2. We used all photographs taken by the camera traps to estimate species richness of medium-sized to large mammalian hosts (from here on referred to as large mammal richness) and of bird hosts (from here on bird richness), and live-trap data to estimate species richness of small mammalian hosts (from here on small mammal richness) by counting all observed host species per plot. We combined the number of host species that were observed by both cameras and live traps to estimate overall species richness of mammalian hosts (from here on mammal richness).

\section{Pathogen prevalence in ticks}

We collected ticks six times in each plot by blanket-dragging ${ }^{199}$ once every four weeks from April - September using a white cotton cloth of $1 \mathrm{~m}^{2}$. We dragged twenty transects of $10 \mathrm{~m}$ during each visit, resulting in $200 \mathrm{~m}^{2}$. Every $10 \mathrm{~m}$ we collected all nymphs in Eppendorf tubes and stored them at $-20^{\circ} \mathrm{C}$ for molecular analysis. For plots that yielded $<600$ nymphs, all nymphs were tested for pathogen prevalence; in plots that yielded more nymphs, a random subset of 600 nymphs were tested for pathogen prevalence, and when prevalence with B. burgdorferi s.l. was below $8 \%$, an extra set was tested to increase precision.

We used the methods described in Coipan et al. ${ }^{234}$, Jahfari et al. ${ }^{51}$, and Chapter 6 to determine the nymphal infection prevalence (NIP) for Anaplasma phagocytophilum, Borrelia afzelii, B. burgdorferi s.s., B. garinii, B. valaisiana, B. miyamotoi, and Candidatus Neoehrlichia mikurensis. NIP values were multiplied with the density of nymphs to calculate the density of infected nymphs (DIN; $100 \mathrm{~m}^{-2}$ ).

\section{Lyme borreliosis risk in Europe}

We tested for a correlation of tick-borne disease risk at a large spatial scale with host species richness, by comparing Lyme borreliosis incidence data as summarized and standardized for western European countries by Sykes and Makiello ${ }^{56}$ and the number of mammalian host species for $I$. ricinus present in each country. We used the list of host species published by Anderson and Magnarelli ${ }^{145}$ and the presence/absence of species as described in the database of the IUCN ${ }^{257}$ to determine the species richness of mammalian hosts (from here on mammal richness) per country. 


\section{6 | Chapter 7}

\section{Statistical analysis}

Statistical analyses were performed in R 3.2.381. First we confirmed the assumed association of the different pathogens with taxonomic groups of hosts by testing for the occurrence of co-infections of pathogen species that were either transmitted by host species in different taxonomic groups or by host species in the same taxonomic group (birds, rodents and ungulates). We used a chisquare test to check for differences in observed and expected frequencies of co-infections of the different pathogens.

We used generalized linear mixed models (GLMM), as implemented in the gImmADMB package ${ }^{200,201}$, to test for a correlation between reservoir-competent and reservoir-incompetent host abundance and nymphal infection prevalence (NIP), and the density of infected nymphs (DIN). We $\log _{10}$-transformed the passage rates and density estimates to approximate a normal distribution where we added the lowest observed value to parameters containing non-detection of the specific species or group of species. All parameters were standardized by extracting the mean and dividing by two standard deviations ${ }^{223}$ and all models had a random intercept for data acquired in the two different years. We checked for collinearity between the different parameters used in multiple parameter models by calculating the variance inflation factor. Variance inflation factors of all parameters used in these models were below 2 , indicating that collinearity was not a problem ${ }^{224}$.

The analysis consisted of three parts. First, for each pathogen species, we tested for correlations between NIP and the abundance of reservoir-competent and reservoir-incompetent hosts. Second, for each pathogen species, we tested for correlations between DIN and the abundance of reservoir-competent and reservoir-incompetent hosts. We compared AICc values ${ }^{82}$ of alternative models to determine whether taxonomic groups of multiple reservoir competent species explained NIP and DIN better than individual species. Third, we tested for a correlation between the abundance of the most important reservoir-competent and reservoir-incompetent hosts and host species richness.

We used GLMMs with a binomial distribution and logit link function, to study the correlation between the abundance of reservoir-incompetent hosts and NIP (amplification effect). The number of tested nymphs with and without infection per plot was used to get a weighted test for the infection prevalence. To test for a correlation between DIN and the abundance of reservoir-competent hosts (amplification effect), we used GLMMs with a negative binomial distribution 
and log link function (as counts of nymphs were over-dispersed). We tested for correlations of Bank vole, Wood mouse, rodent and small mammal density with NIP and DIN for B. afzelii, B. miyamotoi, and Ca. N. mikurensis; for correlations of Blackbird, common thrush and reservoir-competent bird abundance with NIP and DIN for B. garinii, and B. valaisiana; for correlations of Red squirrel, Blackbird, common thrush and reservoir-competent bird abundance, and Bank vole, Wood mouse, rodent and small mammal density with NIP and DIN for B. burgdorferi s.s.; and for correlations of Roe deer, deer and ungulate abundance with NIP and DIN for A. phagocytophilum. We calculated AICc values ${ }^{82}$ to compare the fit of the different models. We considered models to perform equally well when $\triangle \mathrm{AICC}$ $<2^{82}$.

We used the best-fitting model for each pathogen to subsequently test for a dilution effect of reservoir-incompetent host species on NIP and DIN. We checked if adding reservoir-incompetent host species improved model fit by comparing AICc values. We added the abundances of reservoir-incompetent

A. phagocytophilum
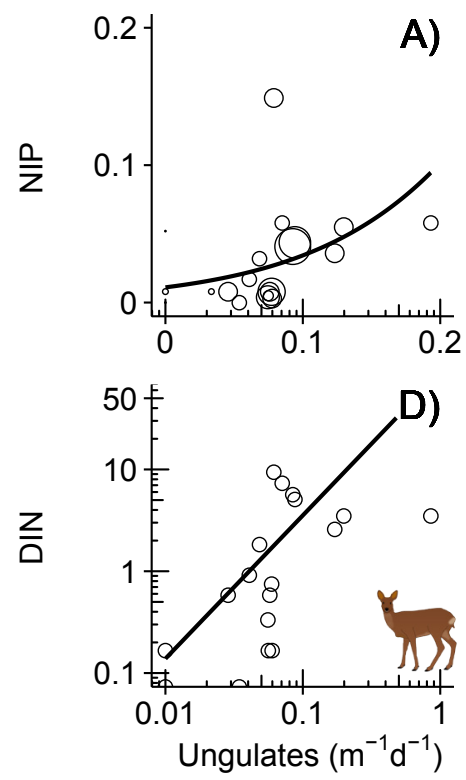

B. garinii
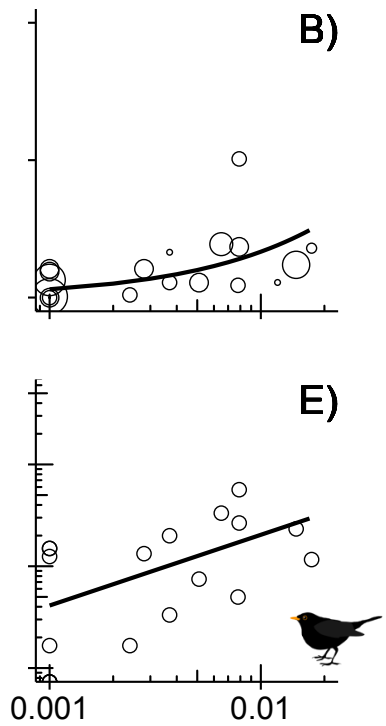

Blackbirds $\left(\mathrm{m}^{-1} \mathrm{~d}^{-1}\right)$
Ca. N. mikurensis

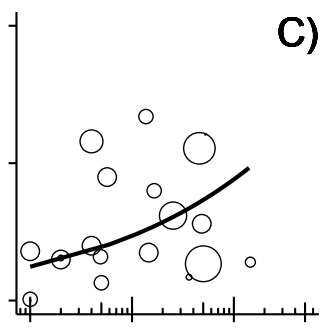

C)

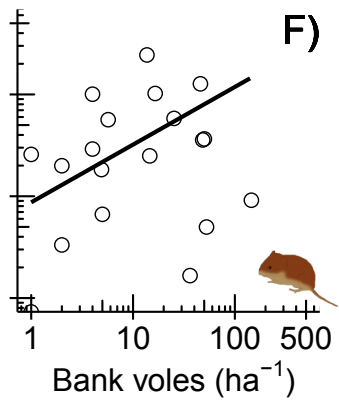

Figure 7.1. Correlations of nymphal infection prevalence (NIP; nymph ${ }^{-1}$ ) and density of infected nymphs (DIN; $100 \mathrm{~m}^{-2}$ ) with host abundance for A/D) Anaplasma phagocytophilum and ungulates $\left.\left(+0.01 \mathrm{~m}^{-1} \mathrm{~d}^{-1}\right), \mathrm{B} / \mathrm{E}\right) \mathrm{B}$. garinii and Blackbirds $\left(+0.001 \mathrm{~m}^{-1} \mathrm{~d}^{-1}\right)$, and C/F) Candidatus Neoehrlichia mikurensis and Bank voles $(+1$ ha-1). The lines are the predictions of the fitted models. Solid lines represent model predictions $(p<0.05)$. Symbol size for NIP scales with sample size. 


\section{8 | Chapter 7}

species separately for all medium-sized to large mammals and birds combined, and for all small mammals combined.

We used linear mixed models (LMMs) as implemented in the nlme package ${ }^{236}$ to test for correlations between the abundance of the most important reservoircompetent and reservoir-incompetent hosts and host species richness. A random intercept per year was included in all models. We used the outcome of the first analyses to determine which species or group of species were included in the model. We tested for a correlation of mammal richness with Lyme borreliosis incidence at a large spatial scale using a GLM with a negative binomial distribution and log link function, as Lyme borreliosis incidence data were over-dispersed.

\section{Results}

Co-infections of pathogen species confirmed the assumed associations of pathogen species with taxonomic groups of hosts. We found a co-infection with at least two pathogen species in $2 \%$ of all tested nymphs (249 of 14,044 tested nymphs), and co-infection with three pathogen species in eight nymphs (0.06\%). We did not find any co-infections of Borrelia garinii and B. valaisiana and therefore combined the numbers of nymphs infected with both pathogen species (bird-transmitted Borrelia spp.) in our tests. Co-infections of birdtransmitted Borrelia spp. and Anaplasma phagocytophilum, of bird-transmitted Borrelia spp. and Candidatus Neoehrlichia mikurensis, and of $\mathrm{Ca}$. N. mikurensis and $A$. phagocytophilum occurred significantly less than expected under random co-occurrence (Table 7.2). In contrast, co-infection of $B$. afzelii and B. miyamotoi and of $B$. afzelii and $C a$. N. mikurensis occurred significantly more than expected (Table 7.2).

Nymphal infection prevalence (NIP) and density of infected nymphs (DIN) Borrelia lusitaniae and B. spielmanii were only found in one questing nymph and were therefore not considered in the statistical analyses. NIP increased with the abundance of reservoir-competent hosts (amplification effect) for six out of seven pathogens (Figure 7.1; Table 7.3). The abundance of taxonomic groups of host species explained NIP better than did the abundance of single reservoircompetent host species for three pathogens: A. phagocytophilum (ungulates), B. burgdorferi s.s. (birds), and B. valaisiana (common thrushes; Table S7.3). After correcting for the abundance of reservoir-competent hosts, NIP decreased 


\section{No Support for a Dilution Effect of Host Species Richness | 129}

Table 7.3. Coefficient estimates of the generalized linear mixed models with the best model fit testing for correlations between the abundance of reservoir-competent and reservoir-incompetent hosts and nymphal infection prevalence (NIP) and density of infected nymphs (DIN).

\begin{tabular}{|c|c|c|c|c|c|}
\hline \multirow{2}{*}{$\begin{array}{l}\text { Pathogen } \\
\text { species }\end{array}$} & \multirow{2}{*}{ Model } & \multicolumn{4}{|c|}{ Coefficient estimate $^{\mathrm{ab}}$} \\
\hline & & Competent host 1 & Competent host 2 & Incompetent host 1 & Incompetent host 2 \\
\hline
\end{tabular}

Anaplasma phagocytophilum

\section{Ungulates}

$\begin{array}{ll}\text { NIP } & 1.2 * * * \\ \text { DIN } & 3.0 * * *\end{array}$

Borrelia afzelii

Wood mice

NIP $0.4 * *$

$-0.9 * * *$

Bank voles

DIN $\quad 0.9$

B. burgdorferi s.s.

$\begin{array}{lll} & \text { Birds } & \text { Red squirrels } \\ \text { NIP } & 1.2 * * & 1.1 * * * \\ \text { DIN } & 1.3 & \end{array}$

B. garinii

\section{Black birds}

$\begin{array}{ll}\text { NIP } & 2.0 * * * \\ \text { DIN } & 1.5 *\end{array}$

B. valaisiana

Common thrushes

$\begin{array}{ll}\text { NIP } & 2.1 * * * \\ \text { DIN } & 1.3\end{array}$

B. miyamotoi

$$
\text { Bank voles }
$$

$\begin{array}{ll}\text { NIP } & -0.1 \\ \text { DIN } & 0.4\end{array}$

Candidatus Neoehrlichia mikurensis

Bank voles
NIP
$0.8 * * *$
DIN
1.7 *
$-0.5 * * *$

a Species or taxonomic group of species presented here are the ones that best explained NIP and DIN based on AICc values (Table S7.3). Reservoir-incompetent hosts are split into two groups, where (1) is the abundance of all incompetent hosts as determined with camera traps and (2) the abundance of all incompetent hosts as determined with live traps (Table S7.2).

b. $p<0.10, * p<0.05, * * p<0.01, * * * p<0.001$. 

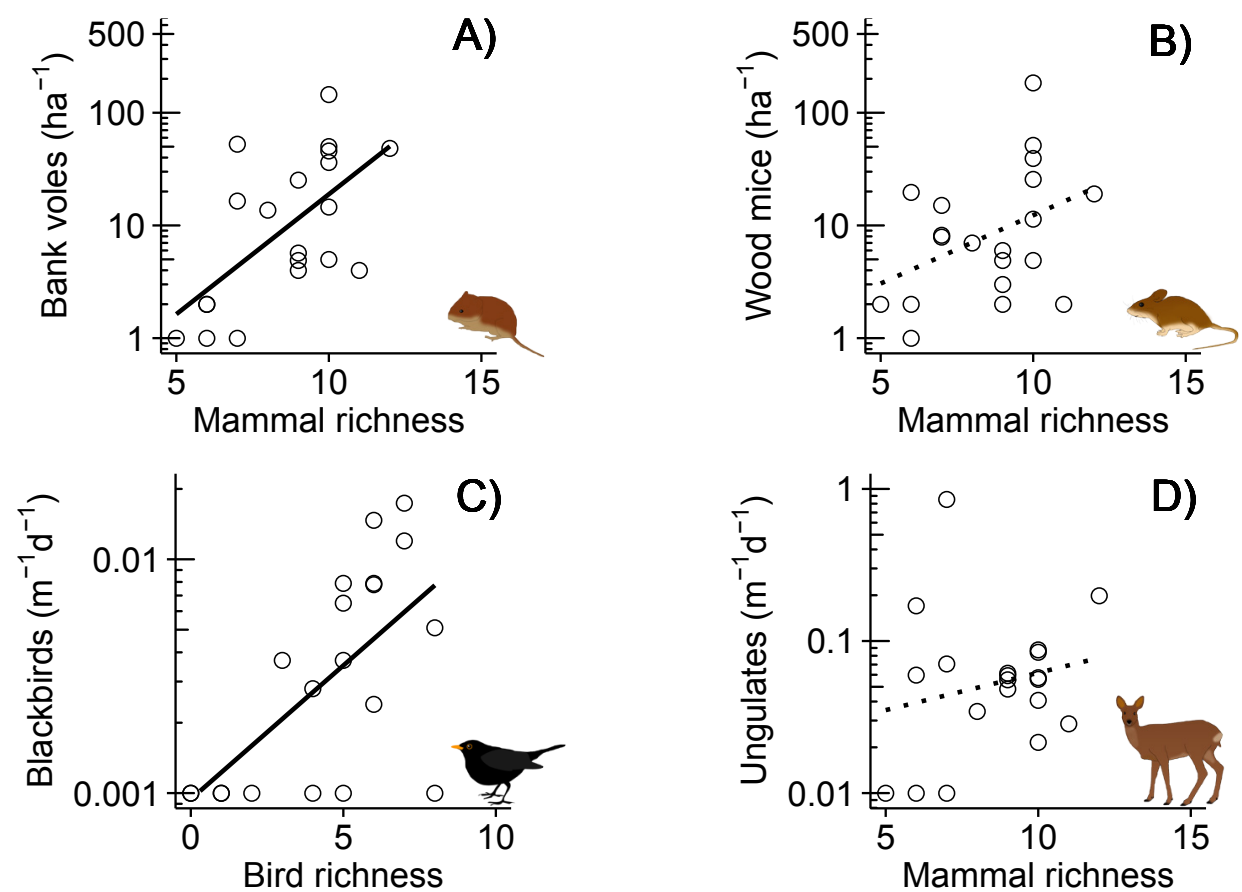

Figure 7.2. Correlations of abundance and host species richness for A) Bank voles $\left(+1\right.$ ha $\left.^{-1}\right)$ and mammal richness, B) Wood mice (+1 ha $\left.{ }^{-1}\right)$ and mammal richness, C) Blackbirds (+0.001 $\left.\mathrm{m}^{-1} \mathrm{~d}^{-1}\right)$ and bird richness, and D) ungulates $\left(+0.01 \mathrm{~m}^{-1} \mathrm{~d}^{-1}\right)$ and mammal richness. Solid lines represent significant correlations $(p<0.05)$, dotted lines non-significant correlations.

with the abundance of reservoir-incompetent hosts (dilution effect) for four pathogen species and increased with the abundance of reservoir-incompetent hosts (amplification effect) for two pathogen species (Tables 7.3 \& S7.4). $\triangle$ AICc values for all tested models on which we based model selection are presented in Table S7.3 (for selection of host species or taxonomic group) and Table S7.4 (for determining model fit of models including the abundance of reservoirincompetent hosts).

We found a positive correlation between the abundance of reservoir-competent hosts and DIN for three pathogens (A. phagocytophilum, B. garinii, and Ca. N. mikurensis; Figure 7.1), but not for the four other pathogens (Table 7.3). Adding the abundance of reservoir-incompetent hosts did not increase the model fit for any of the models describing DIN (Table S7.4). 
Host species abundance and species richness

The density of Bank voles rose with mammal richness $(\beta=0.2, p=0.004$; Figure $7.2 A)$, while the density of Wood mice did not $(\beta=0.05, p=0.32$; Figure 7.2B). The abundance of Blackbirds rose with bird richness $(\beta=0.1, p=0.003$; Figure $7.2 \mathrm{C}$ ) as did the abundance of common thrushes ( $\beta=0.1, p=0.003)$, and reservoir-competent birds $(\beta=0.2, p<0.001)$. The abundance of Red squirrels was not correlated with mammal richness $(\beta=0.08, p=0.18)$ and neither was ungulates abundance $(\beta=0.05, p=0.38$; Figure 7.2D).

The abundance of reservoir-incompetent hosts for $A$. phagocytophilum rose with total host species richness ( $\beta=0.09, p=0.003$ ). In contrast, the abundance of hosts that are reservoir incompetent for $B$. afzelii was not correlated with total host species richness ( $\beta=0.03, p=0.38$ ), and neither were the abundances of reservoir-incompetent hosts for $B$. garinii and $B$. valaisiana $(\beta=0.04, p=0.21$ ), B. burgdorferi s.s. ( $\beta=0.03, p=0.33$ ) or $B$. miyamotoi and Ca. $N$. mikurensis ( $\beta$ $=0.04, p=0.26$ ).

\section{Lyme borreliosis incidence in Europe}

The incidence of Lyme borreliosis in 17 countries in Western Europe increased with mammal richness $(\beta=0.08, p=0.02$; Figure 7.3). Two countries show an opposite trend as both Portugal $($ LB incidence $=0.04$, Mammal richness $=$ 28 ) and Italy (LB incidence $=0.001$, Mammal richness $=44$ ) have a relatively low incidence of Lyme borreliosis compared to the number of mammalian host species.

\section{Discussion}

The dilution effect of host species richness hypothesis, which states that pathogen prevalence should decrease with species richness ${ }^{3,34}$ is based on two conditions which have hardly been tested empirically ${ }^{258}:$ 1) pathogen prevalence increases with the relative abundance of reservoir-competent compared to reservoir-incompetent host species, and 2) the relative abundance of reservoircompetent host species decreases with species richness. We empirically studied the relationship between tick-borne disease risk and host species richness at two spatial scales. We found that the nymphal infection prevalence (NIP) for six pathogen species and the density of infected nymphs (DIN) for three pathogen species increased with the abundance of reservoir-competent hosts, and NIP 


\section{2 | Chapter 7}

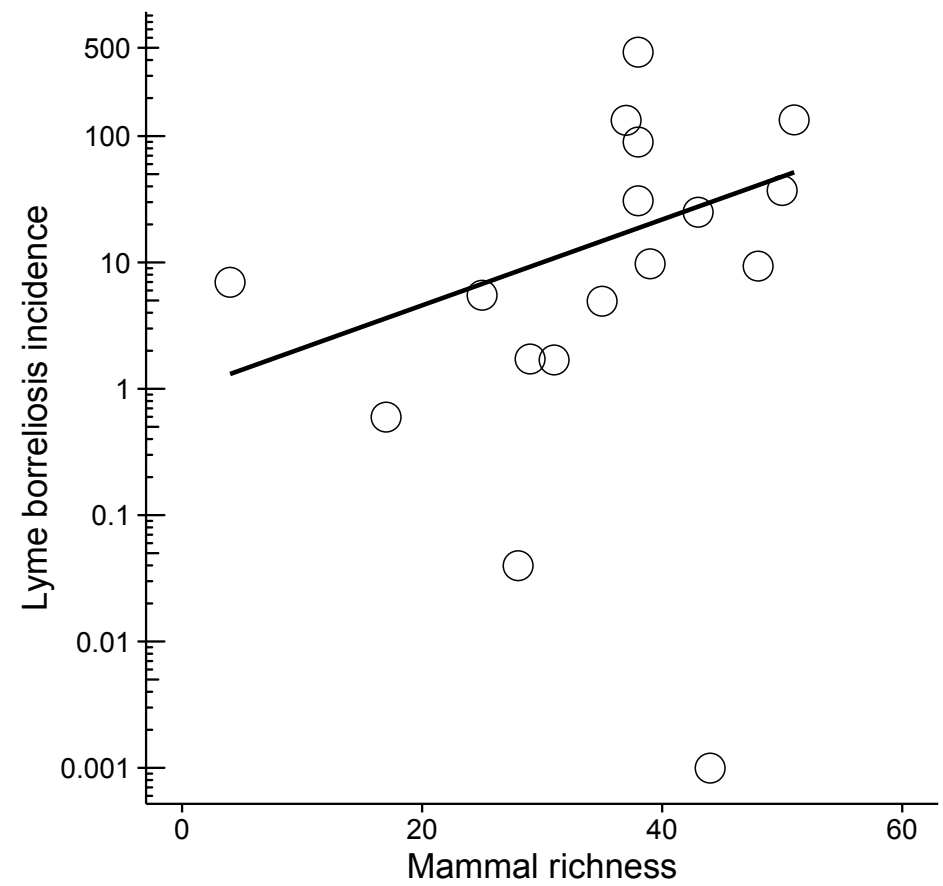

Figure 7.3. Correlation of Lyme borreliosis incidence (annual number of new cases per 100,000 inhabitants) and mammal host species richness for 17 countries in Western Europe. The line shows the fit for the generalized linear model.

decreased with abundance of reservoir-incompetent hosts for four pathogen species across twenty forest plots within the Netherlands, supporting the first condition for a dilution effect of host species richness. However, the abundance of two of the most important host species for Borrelia burgdorferi s.l. in Europe (Bank vole and Blackbird) ${ }^{190}$ (Chapter 2), increased with host species richness in these forests, while the abundance of species that are reservoir-incompetent hosts for six pathogens did not, contradicting the second condition for a dilution effect of host species richness. At a larger spatial scale, Lyme borreliosis incidence increased with species richness of mammalian hosts for Ixodes ricinus across 17 countries in Western Europe. These findings indicate that there is no dilution effect of host species richness for tick-borne pathogens in Europe. Rather, the abundances of reservoir-competent and reservoir-incompetent hosts appear to be the most important determinants of tick-borne disease risk in Europe.

NIP increased with reservoir host abundance (amplification effect) for six out of seven pathogen species. Thus the abundance of reservoir-competent hosts 
appears to be an important determinant of NIP for tick-borne pathogens (Figure 7.1). To our knowledge, this study is the first to find a relationship between reservoir-competent host abundance and NIP in Europe, as evidence so far was circumstantial. One study found a significant difference in A. phagocytophilum prevalence between five sites that also differed in deer abundance ${ }^{259}$, while other studies related differences in tick-borne pathogen prevalence to differences in vegetation structure or landscape configuration, which might be related to reservoir-competent host abundance ${ }^{253,260,261}$. We did not find a correlation between reservoir-competent host abundance and NIP for B. miyamotoi, which might be explained by trans-ovarial transmission ${ }^{43}$.

We found that NIP for four pathogen species decreased with reservoirincompetent host abundance (dilution effect), after correcting for reservoircompetent host abundance, confirming predictions from theoretical models (e.g., Van Buskirk and Ostfeld ${ }^{27}$ ). This is in line with previous studies in Europe that found a dilution effect of deer abundance on NIP for Borrelia burgdorferi s.l. ${ }^{259,262,263}$. In contrast, NIP for the two B. burgdorferi s.l. genospecies that are transmitted by birds ( $B$. garinii and $B$. valaisiana) increased with abundance of reservoir-incompetent hosts. This unexpected result could be caused by an increase in tick burden on thrushes with increasing reservoir-incompetent host abundance, as both larval burden and infection prevalence in thrushes will increase with tick burden ${ }^{190}$ (Chapter 2). Little is known about the determinants of tick burden on birds. Marsot et al. ${ }^{18}$ and Heylen et al. ${ }^{264}$ propose that the foraging behaviour of birds is an important determinant of tick burden. Studies investigating determinants of tick burden on birds, and especially Blackbirds, in relation to other (mammalian) hosts are needed to better understand the determinants of NIP for bird-transmitted tick-borne pathogens.

We found mixed results for correlations of DIN with host abundance across forests. We found a positive correlation between reservoir-competent host abundance and DIN for three pathogen species (A. phagocytophilum, B. garinii, and Ca. N. mikurensis; Figure 7.1), but not the four other species. In Chapter 6 we found that DIN increased with median larval burden on rodents for three pathogen species (B. afzelii, B. miyamotoi and Ca. N. mikurensis) in the same forested plots. Differences in tick burden have a large potential impact on DIN as both the number of hosts getting infected by feeding nymphal ticks and the number of infected hosts feeding larvae increase ${ }^{190}$ (Chapters $2 \& 6$ ). Therefore, reservoir-competent host density alone may not be enough to explain differences 


\section{4 | Chapter 7}

in DIN between plots, and differences in tick burden on reservoir-competent hosts should also be taken into account in theoretical models.

Our data support the role of groups of vertebrate host species in amplifying and transmitting different tick-borne pathogens. Co-infections of pathogen species maintained by different taxonomic groups were less common than expected, while pathogen species transmitted by the same taxonomic group were more common than expected (Table 7.3). The only exception was B. burgdorferi s.s., for which NIP increased with the abundance of Red squirrels and birds in our sites.

We found a positive correlation between the abundance of reservoir-competent hosts and host species richness for two species: the Bank vole and Blackbird (Figure 7.2), and two taxonomic groups: common thrushes and reservoir competent birds. These patterns disagree with one of the most important conditions for a dilution effect of host species richness to occur, namely that reservoir-competent hosts are most abundant in species-poor assemblages ${ }^{3}$. The observed correlations are most probably driving the positive correlation between Lyme borreliosis incidence and mammal host species richness across Western Europe (Figure 7.3). Therefore, there is no support for a dilution effect of host species richness for tick-borne pathogens in Europe, either at a large or small spatial scale. Our findings contrast sharply with studies from North America, where there is substantial evidence that the most important reservoir-competent hosts for B. burgdorferi s.s., the White-footed mouse (Peromyscus leucopus) and Eastern chipmunk (Tamias striatus), are omnipresent and most abundant in species-poor assemblages ${ }^{230,265}$. Therefore, although the determinants of NIP and DIN (abundance of reservoir-competent and reservoir-incompetent hosts) are the same on both continents, the correlation of disease risk with species richness is reversed. In other words, the ecology of tick-borne pathogens does not seem to differ between the two continents, but the relationship between the abundance of reservoir-competent hosts and host species richness does. This could be caused by many factors, including differences in persecution history of rare species and landscape configuration ${ }^{266}$ but also a species identity effect. To better understand the ecology of infectious diseases, disease ecologists should therefore study pathogen prevalence in relation to the specific species that can or cannot transmit pathogens, rather than a general parameter such as host species richness.

We used camera traps to study the availability of medium-sized to large 
mammals and birds for questing ticks, a relatively novel approach. While camera traps might not detect all species present in a site ${ }^{231}$, they do yield an estimate of the passage rate of species walking on the ground, which gives a measurement for their actual availability to questing ticks. Passage rate might be a better estimate of host availability for ticks than host density, as it incorporates how much individual hosts move, and therefore the probability of hosts to encounter ticks $^{12}$. Another advantage is that the same camera traps could be used to study the abundance of a large range of both reservoir-competent and reservoirincompetent hosts and to study host species richness. Camera traps are a suitable tool to investigate host assemblages for ticks or other parasites that are encountered by hosts in the environment, although live trapping might still be necessary for small mammals that are hard to detect with camera traps (but see Villette et al. ${ }^{267}$ ).

In summary, we found support for an amplification effect of the abundance of reservoir-competent hosts on the prevalence of six tick-borne pathogen species in questing nymphal $I$. ricinus in forests in the Netherlands, where we found a positive correlation between the abundance of several reservoir-competent host species and host species richness. We found a dilution effect of the abundance of reservoir-incompetent hosts for three tick-borne pathogens, and an amplification effect of reservoir-incompetent host abundance for two tick-borne pathogens. Furthermore, we found that Lyme borreliosis incidence in Western Europe increased with mammal host species richness. Our findings contradict a dilution effect of host species richness for tick-borne pathogens in Europe, both at a small and large spatial scale, and support critiques of the dilution effect arguing that species identity is more important than species richness ${ }^{158}$. We therefore conclude that studies investigating vector-borne disease risk should study the density of reservoir-competent and reservoir-incompetent hosts rather than host species richness as determinant of vector-borne disease risk. 


\section{6 | Chapter 7}

\section{Appendix to chapter 7}

Table S7.1. Estimated Effective Detection Distances (EDD), for the bird species photographed with camera traps.

\begin{tabular}{|c|c|c|}
\hline Species & Body mass $^{a}(\mathrm{~g})$ & $\operatorname{EDD}(m)^{b}$ \\
\hline Chaffinch (Fringilla coelebs) & 22 & 1.31 \\
\hline Eurasian blackbird (Turdus merula) & 97 & 1.45 \\
\hline Eurasian blue tit (Cyanistes caeruleus) & 11 & 1.25 \\
\hline Eurasian jay (Garrulus glandarius) & 160 & 1.50 \\
\hline Eurasian siskin (Spinus spinus) & 13 & 1.26 \\
\hline Eurasian woodcock (Scolopax rusticola) & 250 & 1.55 \\
\hline European starling (Sturnus vulgaris) & 80 & 1.43 \\
\hline Great spotted woodpecker (Dendrocopos major) & 80 & 1.43 \\
\hline Great tit (Parus major) & 19 & 1.29 \\
\hline Mistle thrush (Turdus viscivorus) & 130 & 1.48 \\
\hline Redwing (Turdus iliacus) & 60 & 1.40 \\
\hline Song thrush (Turdus philomelos) & 74 & 1.42 \\
\hline Stock dove (Columba oenas) & 300 & 1.57 \\
\hline Wood pigeon (Columba palumbus) & 457 & 1.61 \\
\hline
\end{tabular}

${ }^{a}$ Body mass values are taken from Cramp and Perrins ${ }^{79}$.

${ }^{\mathrm{b}}$ Estimates of EDD are based on a point model with a hazard-rate detection probability function with $\log _{10}{ }^{-}$ transformed body mass as covariate. 
No Support for a Dilution Effect of Host Species Richness | 137

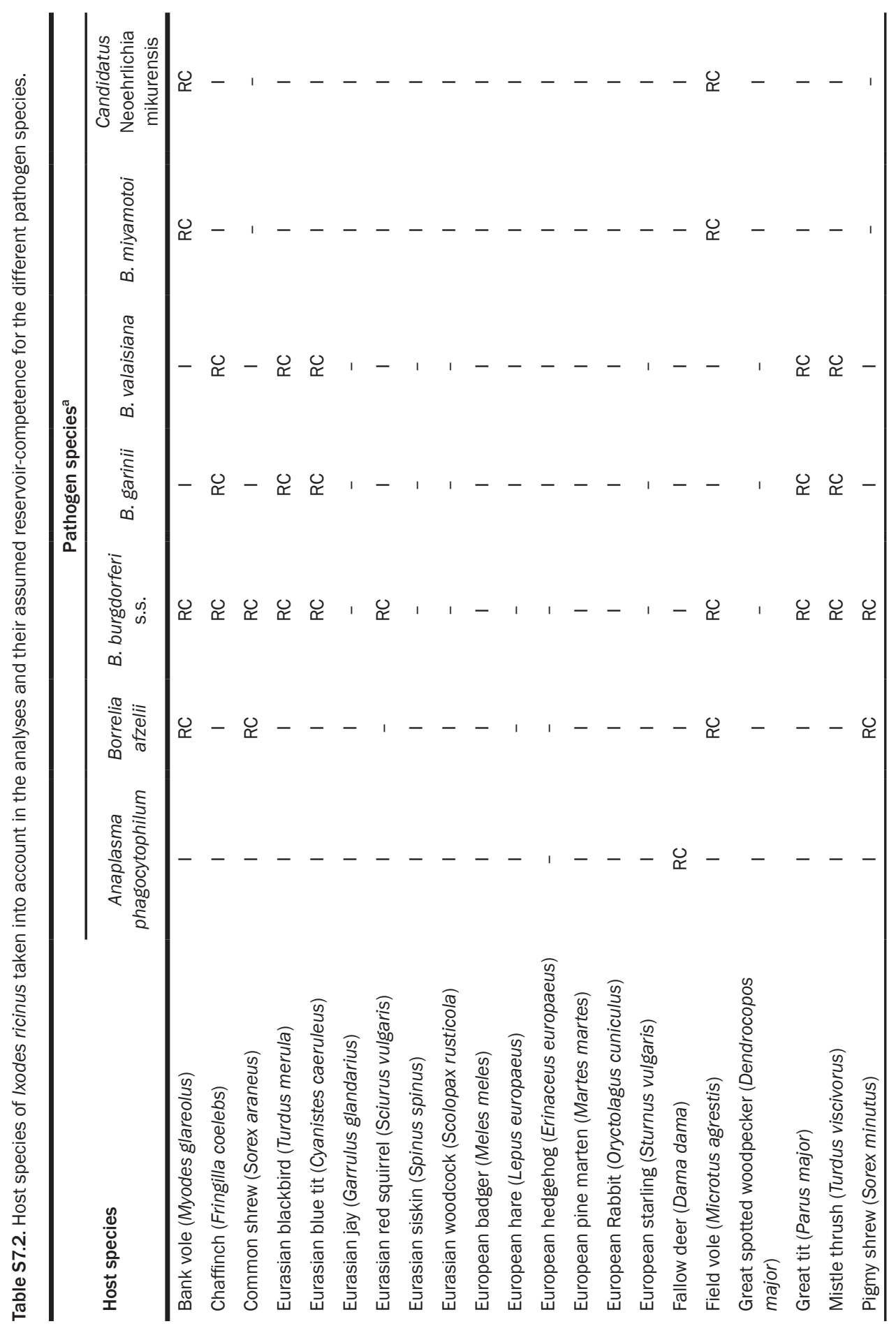


138 | Chapter 7

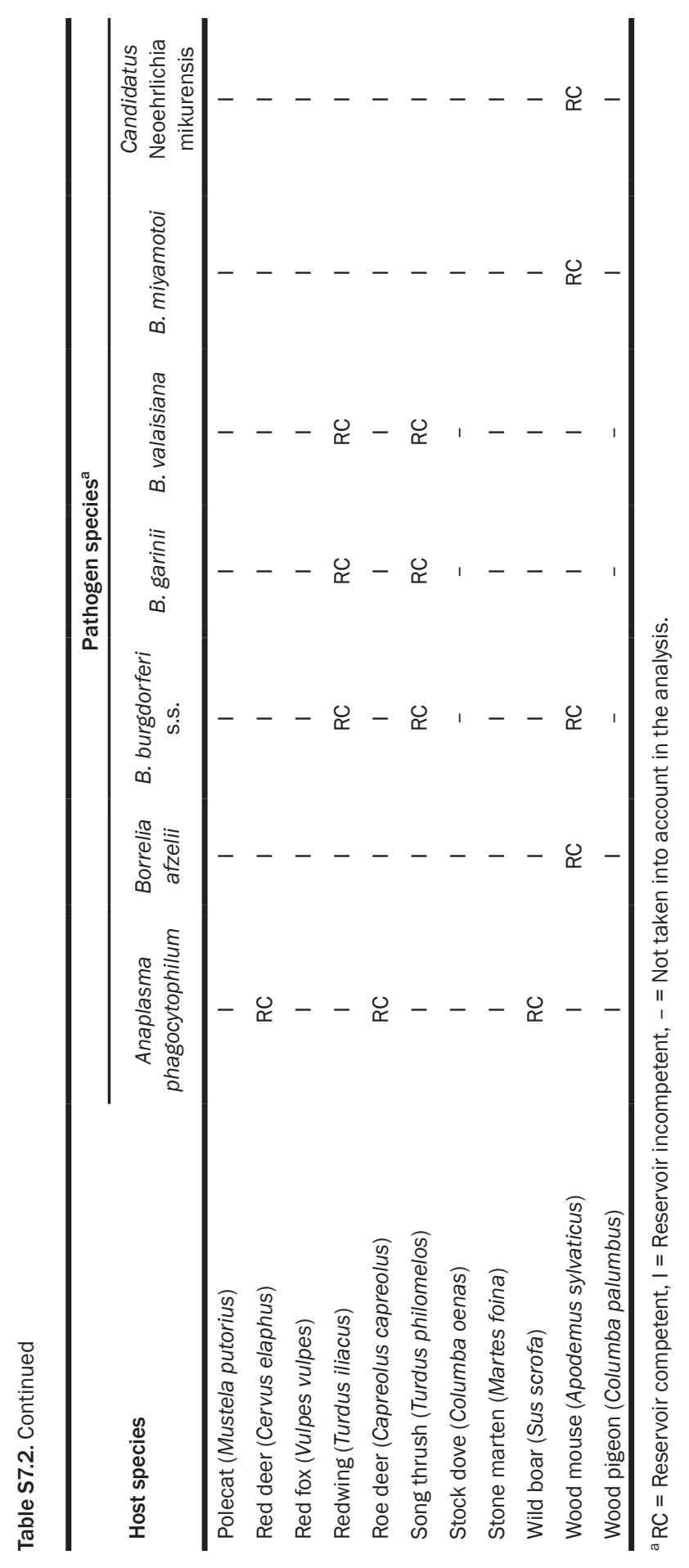


No Support for a Dilution Effect of Host Species Richness | 139

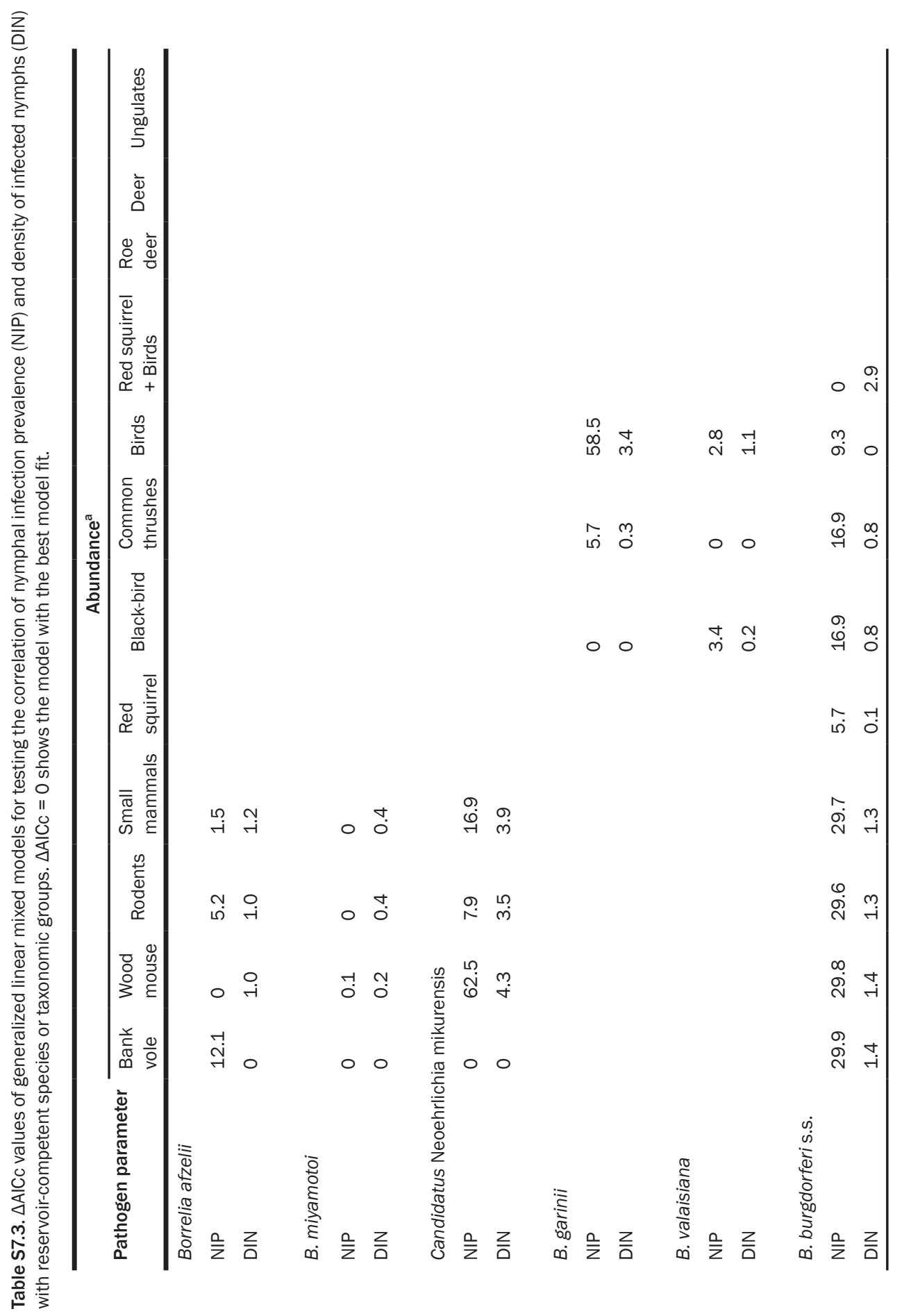




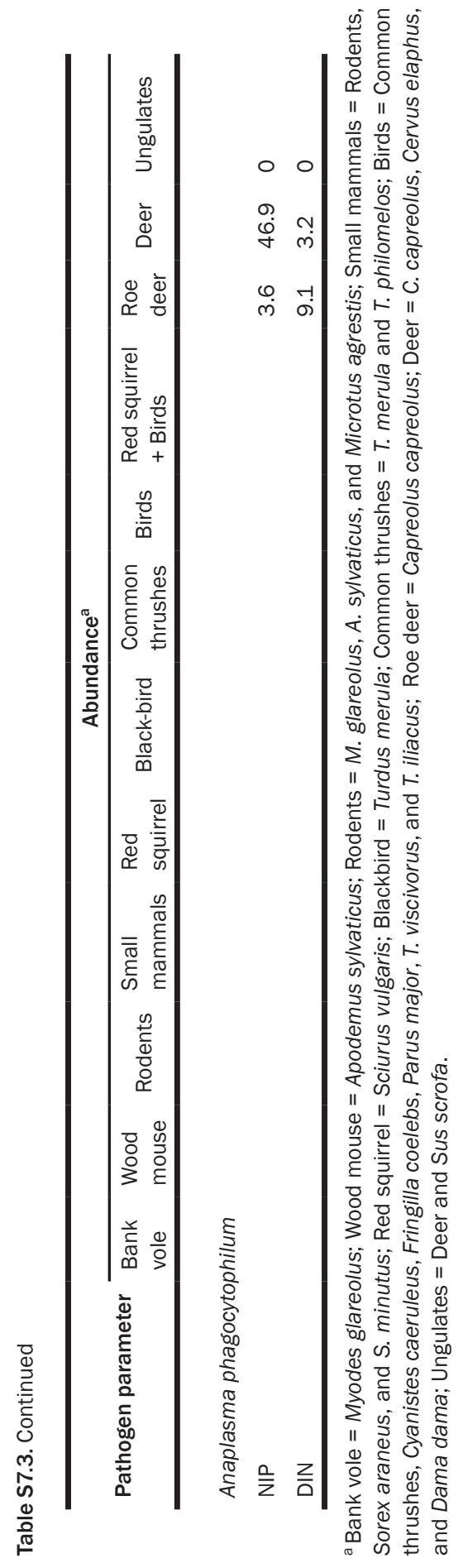




\section{No Support for a Dilution Effect of Host Species Richness | 141}

Table S7.4. $\triangle \mathrm{AICC}$ values of generalized linear mixed models for testing the correlation of nymphal infection prevalence (NIP) and density of infected nymphs (DIN) with abundance of reservoir-competent hosts (Model 1) and the same model with the abundance of reservoir-incompetent hosts added (Model 2). $\Delta$ AICc $=0$ shows the model with the best model fit.

\begin{tabular}{llc}
\hline \multirow{2}{*}{ Pathogen parameter } & \multicolumn{2}{c}{ Model } \\
\cline { 2 - 3 } & $1^{\mathrm{a}}$ & 2 \\
\hline Anaplasma phagocytophilum & & \\
NIP & 110.1 & 0 \\
DIN & 0 & 0.9 \\
Borrelia afzelii & & \\
NIP & 33 & 0 \\
DIN & 0 & 3.5
\end{tabular}

B. burgdorferi s.s.

$\begin{array}{lll}\text { NIP } & 0 & 2.4 \\ \text { DIN } & 0 & 2.8\end{array}$

B. garinii

NIP

11.50

DIN

$\begin{array}{ll}0 & 0.4\end{array}$

B. valaisiana

NIP

3.10

DIN

$0 \quad 1.4$

B. miyamotoi

NIP

20

DIN

$0 \quad 3.6$

Candidatus Neoehrlichia mikurensis

\begin{tabular}{lll} 
NIP & 10.3 & 0 \\
DIN & 0 & 3.5 \\
\hline
\end{tabular}

a Reservoir-competent hosts used in the models were: ungulates (Fallow deer Dama dama, Red deer Cervus elaphus, Roe deer Capreolus capreolus and Wild boar Sus scrofa) for A. phagocytophilum; Wood mouse (Apodemus sylvaticus) for B. afzelii NIP and Bank vole (Myodes glareolus) for B. afzelii DIN; Eurasian red squirrel (Sciurus vulgaris) and birds (Blue tit Cyanistes caeruleus, Chaffinch Fringilla coelebs, Eurasian blackbird Turdus merula, Great tit Parus major, Mistle thrush T. viscivorus, Redwing T. iliacus, and Song thrush T. philomelos) for B. burgdorferi s.s. NIP and birds for DIN; Eurasian blackbird for B. garinii; Eurasian blackbird and Song thrush for B. valaisiana; and Bank vole for B. miyamotoi and Ca. N. mikurensis. 


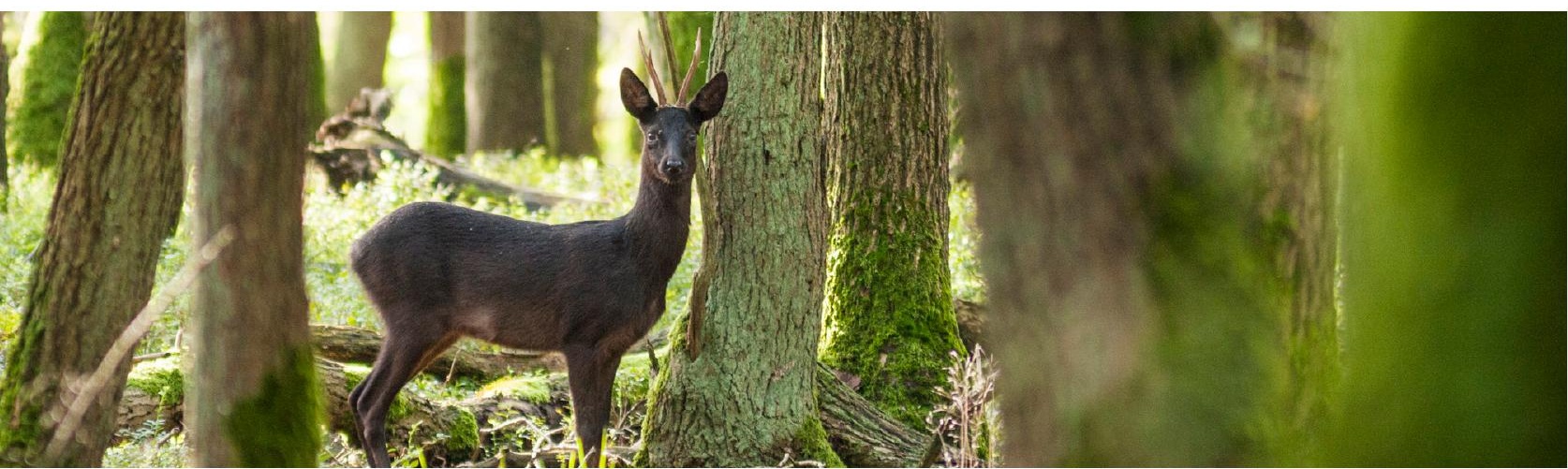

take some mice

nicely sliced

add a deer, or two

heat it up and brew

grind a tick

do it quick

blend it with some dew

let us make a stew

season with a bird

more things it will disturb

stir it through and through

let it brew and brew

taste it well

can you foretell

if the value of

your recipe comes true?

Ronald Hofmeester 


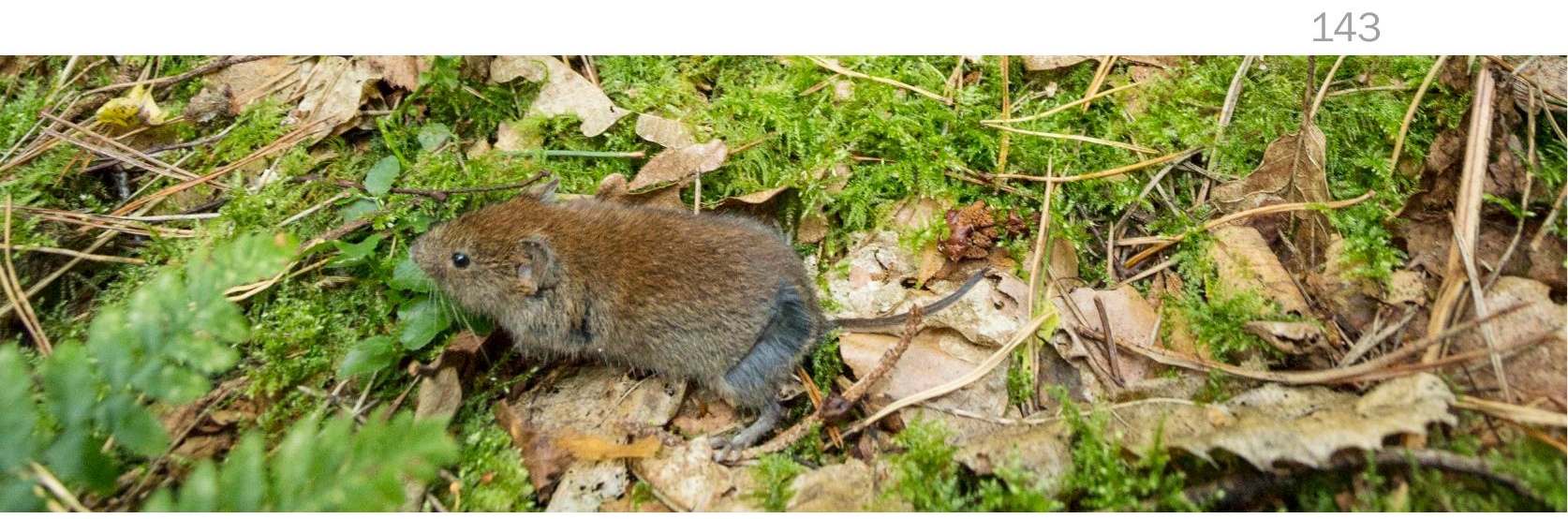

\section{SYNTHESIS: From Biodiversity to BehaVIOUR OF Specific Species in Disease Ecology}

Zoonotic vector-borne diseases have increased in incidence in the last decades ${ }^{2}$. In Europe and North America, tick-borne pathogens cause the majority of vector-borne diseases, including Lyme borreliosis, Rocky Mountain spotted fever and tick-borne encephalitis ${ }^{57}$. Due to the complex life cycle of tick-borne pathogens including multiple vertebrate host and vector species, these pathogens form a challenge to ecologists. By accepting this challenge, ecologists can begin to understand what drives dynamics in microbial populations with the added benefit of obtaining knowledge on the micro-organisms that cause disease in humans. By reviewing the knowledge on the most studied tick-borne pathogen, Borrelia burgdorferi sensu lato (s.I.) in Europe (Chapter 2), I could pinpoint the gaps in our knowledge on this well-studied system. The outcomes of the review provided the basis for a large cross-sectional study and an exclosure experiment. By performing these studies I gained a better understanding of the role of different vertebrate hosts in 1) maintaining Sheep tick (Ixodes ricinus) populations (Chapters 4 \& 5), and 2) infecting ticks with multiple tick-borne pathogens (Chapters 6 \& 7). My findings result in a better understanding of the intricate interplay between vertebrate hosts, l. ricinus and tick-borne pathogens. However, much is still unknown. In this synthesis, I discuss what new insights the results from my thesis bring and how they might change theory on the influence of vertebrate hosts on disease dynamics. I first discuss my findings on tick-borne pathogens in the Netherlands and relate these to previous studies on the relationship between vertebrate hosts and tick-borne pathogen prevalence. Second, I present a mathematical model in which I show the importance of spatial behaviour of hosts for tick-borne pathogen dynamics and proclaim the need for integration of models and theory from the field of behavioural ecology into disease ecology. Concluding, I will speculate about future developments of vertebrate populations in Europe and the influence these will have on tick-borne disease risk. 


\section{4 | Chapter 8}

\section{The idiosyncratic link between biodiversity and tick-borne disease risk}

Until recently, many studies investigating the ecology of infectious diseases focussed on one pathogen transmitted by one host species ${ }^{268}$. However, many pathogens are maintained by multiple host species in natural systems, where host species often differ in their ability to maintain and transmit the pathogen (reservoir competence) ${ }^{4}$. For pathogens that are maintained by multiple host species, the addition or loss of a host species in an area can result in an increase (amplification) or a decrease (dilution) of pathogen prevalence and abundance, depending on the identity of the species ${ }^{269}$. Ostfeld and Keesing ${ }^{157}$ were among the first to study the correlation between host assemblage composition and disease risk, and showed that Lyme borreliosis incidence decreased with the species richness of small-mammal hosts in the north-eastern U.S.A., which led to the proposition of the dilution effect of host species richness hypothesis. The dilution effect hypothesis states that disease risk should decrease with increasing biodiversity. For vector-borne diseases the system has to comply to four conditions in order for a dilution effect to occur ${ }^{3}: 1$ ) the vector must be a generalist, feeding on multiple host species 2) a substantial number of infected vectors must acquire the infection from an infected host, 3) host species must differ in their reservoir competence, and 4) there must be a positive correlation between reservoir competence and numerical dominance in the host assemblage, such that the most competent host species tends to be present in species-rich and species-poor assemblages. However, systems might not always meet these conditions, which has sparked an intense debate over the generality of the dilution effect of host species richness ${ }^{158,270,271}$. A recent meta-analysis concluded that the majority of studies showed a decrease of pathogen prevalence with increasing species richness ${ }^{251}$. Of course this is good news for conservationists trying to convince governments to preserve biodiversity. But it can also lead to false expectations as there are potentially lethal exceptions, such as different hantaviruses and Yersinia pestis, the causative agent of bubonic plague, for which pathogen prevalence increased with species richness ${ }^{251}$. I found a similar result for tick-borne pathogens in Europe (Chapter 7), where disease risk increased with host species richness. More important, the focus on the pattern - disease risk decreases with host diversity - has not brought us closer to understanding the mechanisms by which different host species influence disease risk ${ }^{31,34}$. 
To better understand how different host species can influence disease risk, it is necessary to first understand the determinants of disease risk. For the tickborne pathogens that I studied, these can be split into three groups: 1) the determinants of tick presence and abundance, 2) the determinants of pathogen prevalence in these ticks, and 3) the determinants of people acquiring a tick bite $^{39}$. I will discuss the first two as these are dependent on the composition of the vertebrate assemblage.

Determinants of I. ricinus abundance

The presence of $I$. ricinus is first of all dependent on the right micro-climatic conditions $^{8}$. These conditions are often found in forested habitat and can be used to forecast $I$. ricinus presence ${ }^{272}$. However, there are substantial differences in $I$. ricinus densities between areas with suitable habitat, which are often attributed to differences in host species composition ${ }^{156,253}$. In Chapter 2 I performed a systematic review to determine the host species that are most important in feeding the different stages of $I$. ricinus. I found that tick burden increased with body mass of the host species, except for larval burden on mammals ${ }^{190}$ (Chapter 2). As body mass of mammals decreased with host density, the majority of larvae feed on small mammals which occur in the highest densities (Figure 2.2). By combining average density and tick burden I showed that deer are the most important hosts for the adult stage, while thrushes were the most important hosts for nymphs (Chapter 2). In light of these findings, I updated Figure 1.1 to show that the majority of all three stages of $I$. ricinus appear to feed on only a few species of vertebrate hosts, belonging to three taxonomic groups: rodents, thrushes and deer (Figure 8.1).

The survival of ticks is dependent on encountering a host ${ }^{8}$, thus $I$. ricinus density per life stage should increase with the abundance of the host species that are most important for feeding the previous life stage. As deer are the most important hosts for adult I. ricinus, larval density should increase with deer abundance. In my study sites larval density was significantly higher in areas with $\operatorname{deer}(n=17)$ compared to areas without deer $(n=3)$, and larval density increased with the relative abundance of deer for which the correlation was strongest in areas where only Roe deer (Capreolus capreolus) were present (Chapter 4). Consequently, larval burden on rodents increased with the relative abundance of deer (Chapters 5-6). As small mammals were found to be the most important hosts for larval I. ricinus (Chapter 2) nymphal densities should increase with 


\section{6 | Chapter 8}

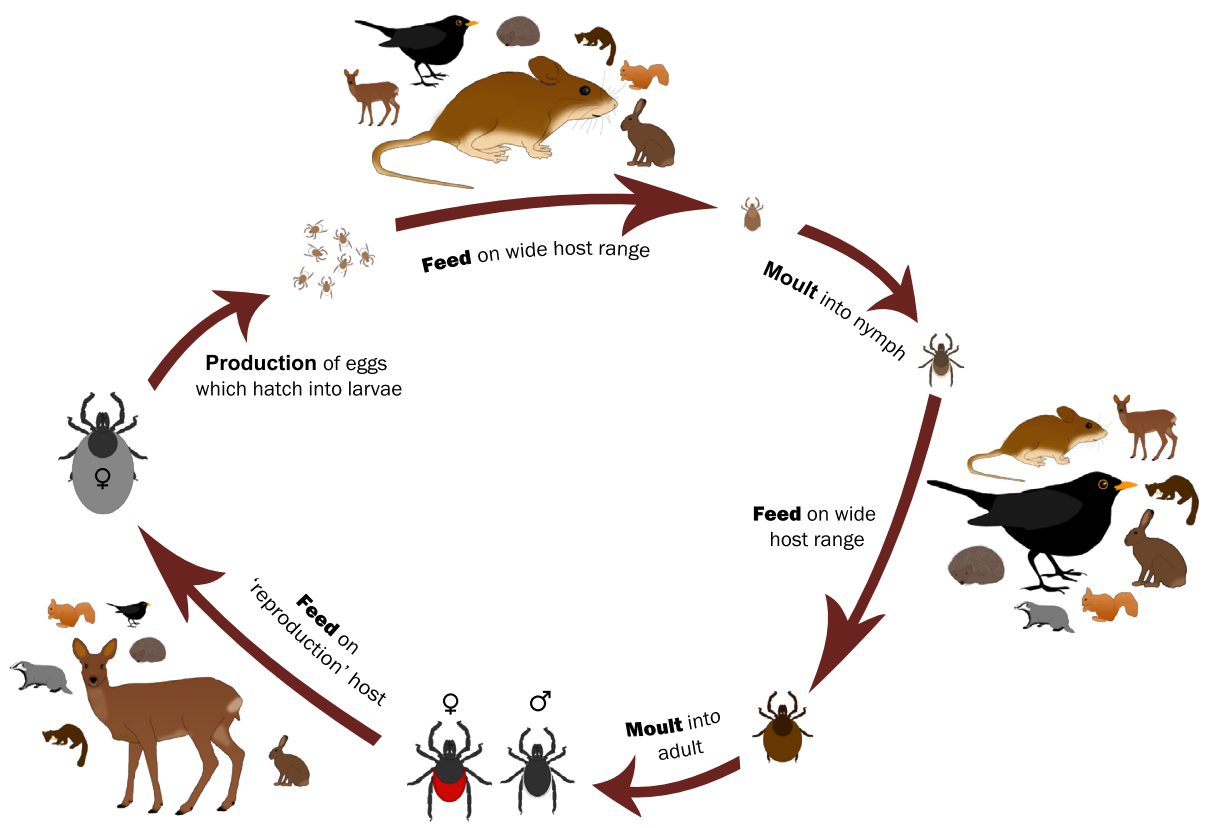

Figure 8.1. Schematic representation of the life cycle of Ixodes ricinus updated with the results from Chapter 2. Although I. ricinus parasitizes many host species, larvae mainly feed on rodents, nymphs on thrushes and adults on deer. Pictures of single species represent groups of taxonomically related species. The size of the host picture represents the theorized importance of the host taxon for that life stage of $I$. ricinus.

small mammal density, which was found for I. scapularis in North America ${ }^{204}$. However, in my study sites there was no correlation between small mammal and tick density (GLMM with negative binomial distribution and log link function and a random intercept per year: $\beta=0.1, p=0.81$ ).

The lack of a correlation of nymphal density and small mammal density in my study sites is most probably caused by the large variation between sites in larval burden on small mammals (Chapter 5). Indeed nymphal density per plot increased with median larval burden on rodents (GLMM with negative binomial distribution and log link function and a random intercept per year: $\beta=1.4$, $p<0.001)$. This suggests that rodents are indeed the most important hosts feeding larvae, and that only their density is not sufficient to predict the density of questing I. ricinus nymphs. To my knowledge there is only one previous study in which rodent density and tick burden were compared to questing I. ricinus density. In a study in Ireland, questing nymph density and larval burden on 
Wood mice (Apodemus sylvaticus) were higher within a forest patch with high deer abundance compared to a patch with low deer abundance, but nymphal density was not correlated to Wood mouse density ${ }^{139}$. However, both Gray et al. ${ }^{139}$ and I determined nymphal density and rodent density in the same year while most nymphs questing in the vegetation fed as larvae in the previous year ${ }^{8}$. It is likely that tick burden on rodents fluctuates less between years than rodent density ${ }^{204}$, which could explain my findings (Chapter 5) and the findings of Gray et al. ${ }^{139}$. Therefore, nymphal density is most probably determined by a combination of rodent density (differences between years) and tick burden on rodents (differences between sites).

In Chapter 5 I found that tick burden on individual rodents was most strongly correlated to the relative abundance of other hosts (predators, deer and rodents). These findings are consistent with studies on two different tick species on two continents. For I. ricinus in Europe, Cagnacci et al. ${ }^{32}$ found an increase of larval burden on rodents with deer abundance in all but one site, and Kiffner et al. ${ }^{95}$ found a decrease of larval burden on rodents with rodent density. Similarly, for I. scapularis in North America, Levi et al. ${ }^{241}$ found that larval burden on rodents decreased with rodent density, and Calabrese et al. ${ }^{12}$ found that larval burden on rodents increased with high larval densities in the environment, most probably related to high densities of hosts for adult ticks. This suggests that the mechanisms determining larval burden on rodents, and subsequently questing nymphal density are similar for both tick species in systems with different host assemblages.

From my results, I conclude that the relative abundance of deer, rodents and predators indirectly determine questing $I$. ricinus nymphal densities, via tick burden on rodents (Chapters 5-6). Although deer abundance was not directly correlated to nymphal density (Chapter 4), the indirect correlation might explain the increase of $I$. ricinus densities with deer abundance found in previous studies $^{38,55,208}$. Especially in locations or habitats where small mammals are scarce, the abundance of deer or thrushes might be an important determinant of nymphal density ${ }^{141,209}$. In my sites, questing adult density increased with questing nymphal density (GLMM with negative binomial distribution and log link function and a random intercept per year: $\beta=1.2, p<0.001$ ), but not with the density of any of the vertebrate hosts (Chapter 4 ). This suggests that questing adult density is mostly determined by the determinants of larval and nymphal densities. Therefore, questing $I$. ricinus density seems to be determined by the relative 


\section{8 | Chapter 8}

abundance of species in multiple taxonomic groups (deer, rodents, thrushes and predators) rather than the abundance of only a single host species.

Determinants of pathogen prevalence in I. ricinus

Theoretical studies have shown that the relative abundances of host species with a high reservoir competence and species with a low reservoir competence determine pathogen prevalence in questing ticks ${ }^{27,33}$. Therefore, it is essential to know which host species have a high reservoir competence. In Chapter 2 I showed that host species that have a high nymphal burden are often infected with $B$. burgdorferi s.I. and subsequently they also have a high realized reservoir competence for $B$. burgdorferi s.I. However, the rodent species that seem to infect most larvae with $B$. burgdorferi s.l. do not have a very high realized reservoir competence. This led me to propose two maintenance strategies for $B$. burgdorferi s.l., namely via a high infection prevalence in ticks (as a consequence of high host density), or via a high infection prevalence in hosts (Chapter 2). In either case, the different genospecies of B. burgdorferi s.l. should evolve to be best transmitted by host species that are often encountered. Thus, the host species that are most important for feeding the different stages of I. ricinus - several species of rodents, thrushes and deer - are also the most likely candidates to be hosts with a high reservoir competence for tick-borne pathogens transmitted by $I$. ricinus. This indeed seems to be the case because rodents are reservoir hosts for B. afzelii, B. bavariensis, B. miyamotoi, and Candidatus Neoehrlichia mikurensis, thrushes for $B$. garinii and B. valaisiana, and deer for Anaplasma phagocytophilum ${ }^{42,50,51,190,233,252}$ (Chapter 2). Therefore, I expected that the prevalence of these tick-borne pathogens would increase with the relative abundance of rodents, thrushes and deer.

In my study sites, infection prevalence in questing I. ricinus nymphs increased with the relative abundance of reservoir-competent hosts for six out of seven studied pathogen species, while infection prevalence decreased with the relative abundance of reservoir-incompetent hosts for four pathogen species, consistent with predictions from theoretical studies ${ }^{27,33}$ (Chapter 7 ). These findings are again consistent with previous findings on two different tick species on two continents. For I. ricinus in Europe, Mysterud et al. ${ }^{263}$ found that infection prevalence of $B$. burgdorferi s.l. in questing nymphs decreased with deer density, as deer are reservoir-incompetent for B. burgdorferi s.I. ${ }^{240}$. For I. scapularis in North America, LoGiudice et al. ${ }^{230}$ showed that a model incorporating densities, larval burden 
and realized reservoir competence of several host species provided predictions of nymphal infection prevalence that were consistent with empirical findings, where nymphal infection prevalence increased with the density of the species with the highest reservoir competence. From these findings I conclude that the relative abundance of specific host species (identity effect) determines the infection prevalence with tick-borne pathogens.

\section{The importance of host identity}

As described above, the determinants of tick-borne disease risk - as defined by the density of ticks and pathogen prevalence in these ticks - are consistent between different systems although the actual species involved differ. It seems, however, that the same mechanisms result in different patterns for the relationship between the prevalence of tick-borne pathogens and host species richness between the I. scapularis system in North America and the I. ricinus system in Europe ${ }^{157,248}$ (Chapter 7). This could be due to the fact that the identity of the species added or lost in an assemblage has a major impact on the predicted pathogen prevalence ${ }^{273}$. For example, the addition of an ungulate species to an assemblage should result in an increase in the prevalence of $A$. phagocytophilum in questing I. ricinus (Chapter 7 ). In contrast, the addition of a mammalian carnivore species to an assemblage should result in a decrease in the prevalence in questing nymphs of $B$. afzelii and other tick-borne pathogen species transmitted by rodents (Chapter 6). Furthermore, species differ in their response to other species in an assemblage, resulting in differences between species for the correlation of host abundance and species richness ${ }^{265}$. Therefore, a broad parameter such as species richness is not a good parameter describing the potential of an assemblage for pathogens, because pathogens are dependent on specific species of hosts and vectors ${ }^{274}$.

Every pathogen species is unique as it is transmitted by its own set of host species - which are themselves also unique - and has its own evolutionary history. Therefore, the identity of pathogens and host species should always be considered when studying pathogen prevalence. Different pathogen species can however show similar patterns. In Chapter 7 I studied seven different pathogen species, which showed similar trends in response to their respective reservoir host species. From this, I conclude that general predictions can be made for tick-borne pathogens, as long as the identities of the pathogen, vector and host species are considered, highlighting the need for studies investigating 


\section{0 | Chapter 8}

the importance of different host species for specific pathogens. This calls for research on the ecology of tick-borne pathogens in systems with I. persulcatus as the most important vector, such as in Russia and the Far East ${ }^{36}$, and other understudied systems.

The study of interactions between specific host species is needed to better understand factors determining pathogen prevalence. There are several theoretical frameworks incorporating different mechanisms by which different host species can influence disease ris $k^{31,34}$. Therefore, I think it is time for disease ecologists to make the next step and start empirical work and experiments on different mechanisms by which vertebrate species can alter the encounter rate between infected and susceptible individuals (for directly transmitted pathogens) or between vectors and reservoir-competent host individuals (for vector-borne pathogens). My work on tick-borne pathogens showed that the density of I. ricinus nymphs infected with different tick-borne pathogens increased with either the larval burden on rodents (Chapter 6) or the relative abundance of Blackbirds (Turdus merula) and ungulates as estimated with camera traps (Chapter 7). Both parameters are related to the spatial behaviour of hosts, as hosts that move more are more likely to encounter ticks or cameras. However, the influence of spatial behaviour of hosts on tick-borne pathogen dynamics is highly understudied.

\section{The importance of behaviour}

Behaviour of hosts has long been recognised as an important determinant of infection prevalence for directly transmitted pathogens, where contact networks are used extensively ${ }^{275}$. Also, it has been shown that host movement can influence parasite distributions on a large spatial scale ${ }^{11}$. However, (spatial) behaviour of hosts is often neglected in studies investigating parasites that are encountered in the environment, especially at small spatial scales. For example, most models describing tick and tick-borne pathogen dynamics in multi-host systems have only considered host density ${ }^{276}$ and even when behaviour was taken into account, it was indirectly modelled as a function of host density ${ }^{277}$. The data in Chapter 5-7 suggest that changes in behaviour of reservoir-competent host species might have strong effects on tick-borne pathogen dynamics, via the average tick burden on hosts. To investigate the effect of behaviour of reservoir-competent hosts and compare this to the effect of host density, I modelled the influence of host density and daily distance travelled on the number of hosts that gets 
Synthesis: From Biodiversity to Behaviour of Specific Species | 151 infected with a tick-borne pathogen and the number of larvae that subsequently get infected by those infected hosts on a small spatial scale (1 ha). I have used the data I obtained for Wood mice and B. afzelii in Chapter 5-7 to parameterize the model.

Modelling the effects of density and daily distance travelled

Most models related to tick and tick-borne pathogen dynamics assume that the influence of different host species on the density of ticks and the prevalence of tick-borne pathogens is determined by the combination of the density, the average tick burden, and the reservoir competence of the different host species in an assemblage ${ }^{27,33,276}$ (Chapter 2). In these models, average tick burden per species is often modelled as a constant, while it is clear that tick burdens on a single species differ between sites with different host assemblages (Chapters 5). There are some indications that these differences are related to spatial behaviour of host individuals ${ }^{12}$ (Chapter 5-7). Therefore, I have modelled a fluctuating tick burden on reservoir-competent hosts as a function of daily distance travelled to investigate the effect of this parameter on tick density and tick-borne pathogen dynamics in relation to host density. As described in Chapter 5 the tick burden on a host is determined by 1) the number of ticks in the environment, 2) the day range of the host and 3 ) the resistance or tolerance (including grooming) of the host. In this model, I assumed that the number of ticks in the environment is equal for all hosts and that resistance or tolerance towards ticks is equal for all host individuals (these and other assumptions made in this model are described in Box 8.1). These two assumptions should be met when considering host individuals from one species (small variation in resistance/tolerance) at a small spatial and temporal scale (small variation in tick density).

Following Calabrese et al. ${ }^{12}$ the expected tick burden, $\lambda$, of a host individual can be described as a function of the daily accumulation of new ticks and the daily loss of ticks that are engorged (see Table 8.1 for a description of all symbols and an overview of the estimates used in this chapter). If I assume that the accumulation of ticks is proportional to the daily distance travelled $M$ (meter . day $\left.{ }^{-1}\right)$, ticks are picked up with a constant rate $\alpha\left(\mathrm{m}^{-1}\right)$, and ticks are lost with a constant rate $\delta\left(\mathrm{d}^{-1}\right)$, daily change in tick burden can be described as,

$$
\frac{d \lambda}{d t}=\alpha \cdot M-\delta \cdot \lambda
$$




\section{2 | Chapter 8}

If I assume that the tick burden found on a host is equal to the equilibrium of the daily change in tick burden $(d \lambda / d t=0)$, it can be described as,

$$
\lambda=\frac{\alpha \cdot M}{\delta}
$$

Equation 8.2

Equation 8.2 can then be used to estimate the larval and nymphal accumulation rates, $\alpha$, and $\alpha_{n}$, respectively, for Wood mice in the Netherlands. I made this distinction to be able to separate 1) the number of Wood mice that gets infected by feeding an infected nymph and 2) the number of Wood mice that subsequently infects feeding larvae. In Chapter 5 I found that the median intensity of infestation with larvae on an average Wood mouse in the Netherlands was 8 and that the prevalence of infestation was 0.84 , resulting in $\lambda_{l}=6.72$. The average $I$. ricinus larva feeds on a rodent host for three days ${ }^{278}$, resulting in $\delta_{1}=1 / 3$ and the average daily distance travelled, $M$, by Wood mice is $350 \mathrm{~m}^{67}$. These numbers result in an estimate of $\alpha_{1}=0.0064$. For Wood mice in the Netherlands, the median intensity of infestation with nymphs was 1 , and the prevalence of infestation was 0.16 , resulting in an average $\lambda_{n}=0.16$ (Chapter 5). Nymphal I. ricinus feed on a rodent host for an average of 3.5 days ${ }^{278}$, giving $\delta_{n}=1 / 3.5$. This results in an estimate of $\alpha_{n}=0.00013$. I used these estimates to model the effect of changes in daily distance travelled, $M$, on larval burden, $\lambda_{l}$, and the probability of infection in Wood mice.

To be able to estimate how many larvae an individual host will infect during its lifetime it is necessary to calculate the cumulative probability that an individual host gets infected over time and the number of larvae it will infect per day if infected. The daily encounter rate with an infected nymph, $I\left(\lambda \cdot \mathrm{d}^{-1}\right)$, can be described as,

$$
I=\alpha_{n} \cdot \iota_{n} \cdot M
$$

Equation 8.3

where $I_{n}$ is the average nymphal infection prevalence with a specific pathogen. If I assume that a host gets infected as soon as it is bitten by an infected nymph, the probability of a host being infected at time $t$ (days), $P l_{(t)}$, can be described as,

$$
P I_{(t)}=1-(1-I)^{t}
$$

Equation 8.4

with $t=1,2, \ldots, L E$, where $L E(d)$ is the average lifespan of a host. I used Equations 
Table 8.1. Summary of the parameters and parameterization for the I. ricinus and Wood mouse model used in this chapter.

\begin{tabular}{|c|c|c|c|}
\hline Symbol & Description (units) & Estimate & Reference \\
\hline$\alpha$ & Larval encounter rate $\left(\mathrm{m}^{-1}\right)$ & 0.0064 & This chapter \\
\hline$\alpha_{n}$ & Nymphal encounter rate $\left(\mathrm{m}^{-1}\right)$ & 0.00013 & This chapter \\
\hline$\delta_{1}$ & Larval detachment rate $\left(\mathrm{d}^{-1}\right)$ & $1 / 3$ & 278 \\
\hline$\delta_{n}$ & Nymphal detachment rate $\left(\mathrm{d}^{-1}\right)$ & $1 / 3.5$ & 278 \\
\hline$D$ & Host density (ha-1) & $10-40$ & Chapter 5 \\
\hline$I_{n}$ & Nymphal infection prevalence $\left(\right.$ nymph $\left.^{-1}\right)$ & 0.052 & Chapter 7 \\
\hline$\lambda_{1}$ & Larval burden on host $^{\mathrm{a}}\left(\right.$ host $\left.^{-1}\right)$ & 6.72 & Chapter 5 \\
\hline$\lambda_{n}$ & Nymphal burden on host ${ }^{\mathrm{a}}\left(\right.$ host $\left.^{-1}\right)$ & 0.16 & Chapter 5 \\
\hline$L E$ & Lifespan of the host (d) & 90 & 279 \\
\hline M & Daily distance travelled $\left(\mathrm{md}^{-1}\right)$ & $175-700$ & 213 \\
\hline s & Survival rate from engorged larva to nymph (larva-1) & 0.4 & 8 \\
\hline
\end{tabular}

a Larval and nymphal burden are the median number of larvae and nymphs, respectively, feeding on an individual host as estimated over all sampled individuals in Chapter 5.

8.3 and 8.4 to estimate the probability that an individual Wood mouse has been infected with $B$. afzelii during its lifetime $\left(P I_{(L E)}\right)$. The average infection prevalence with $B$. afzelii in questing nymphs in the Netherlands is, $I_{n}=0.052$ (Chapter 7 ), which I used to calculate $I \approx 0.0024$. Using the average lifespan of Wood mice, $L E=90$ days $^{279}$, this results in $P l_{(L E)} \approx 0.19$, which is quite similar to the average infection prevalence of 0.17 for $B$. burgdorferi s.I. in Wood mice as found in Chapter 2.

Using equation 8.4, I calculated the cumulative probability of infection with $B$. afzelii for a Wood mouse for a range of values for $M$ that were obtained in a telemetry study on Wood mice in the UK $(25-2500 m)^{213}$, which shows that the probability of infection at time $t$ approximately doubles with a twofold increase in daily distance travelled $M$, until values at the top of the observed range are reached (Figure 8.2).

I calculated the average total number of larvae encountered by one host during 


\section{4 | Chapter 8}

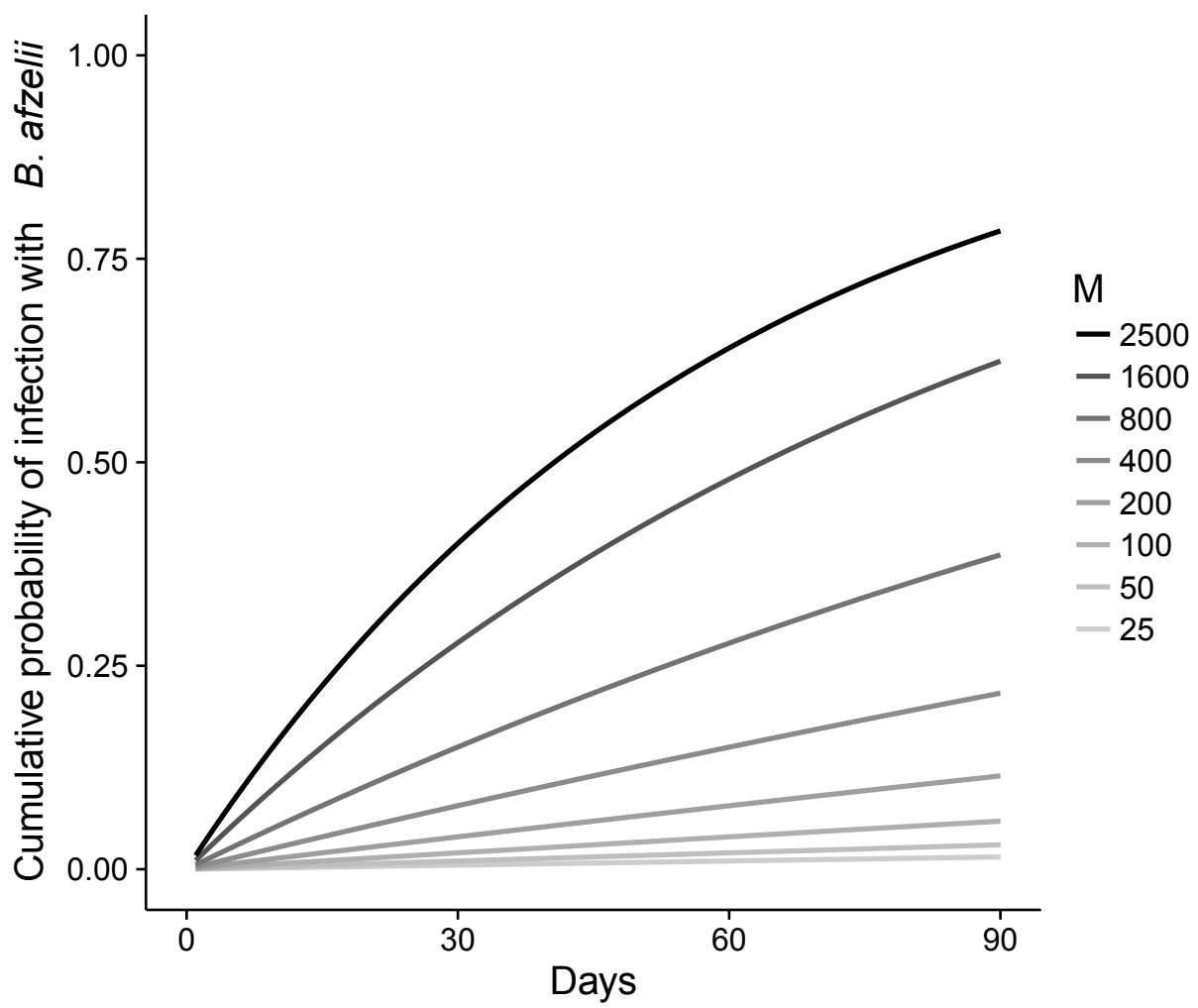

Figure 8.2. Cumulative probability of infection with $B$. afzelii as a function of daily distance travelled, $M$, for Wood mice.

a lifetime, $L$, using the average lifespan and the number of larvae encountered per day as described in equation 8.1 as,

$$
L=L E \cdot \alpha_{l} \cdot M
$$

Using the average Wood mouse data, and equation 8.5, the average number of larvae encountered per Wood mouse during its lifespan is $L \approx 202$. If I assume that the density of hosts $D$ (hectare ${ }^{-1}$ ) is constant during one cohort of individuals with lifespan $L E$, the number of larvae fed by a population of hosts, $L_{p}$, can be described as,

$$
L_{p}=L \cdot D=L E \cdot \alpha_{l} \cdot M \cdot \mathrm{D}
$$

Equation 8.6 
From these equations it follows that the effects of daily distance travelled and host density on $L_{p}$ are the same: a twofold increase in density, or a twofold increase in daily distance travelled will both result in a twofold increase in $L_{p}$ (Figure 8.3).

If I assume that encountered larvae are infected as soon as the host has encountered an infected nymph, the cumulative number of larvae that gets infected by an individual host during its lifespan, $L_{i}$, can be described as,

$$
L_{i}=\sum_{t=1}^{L E} \alpha_{l} \cdot M \cdot\left(1-(1-I)^{t}\right)
$$

Equation 8.7

Using this equation for Wood mice and $B$. afzelii in the Netherlands gives $L_{i} \approx$ 20. By multiplying $L_{i}$ by the survival rate of host attached larva to nymph, $s$, the number of infected nymphs, $N_{i}$, that got their infection from an individual mouse during their lifetime can be described as,

$$
N_{i}=L_{i} \cdot S
$$

Equation 8.8

The average survival rate of host attached larva to nymph, $s$, for $I$. ricinus is $0.4^{8}$, which results in $N_{i} \approx 8$ for $B$. afzelii. To calculate the density of infected nymphs supported per host population, I assume a constant density of hosts $D$ (hectare $^{-1}$ ) during one cohort of individuals with lifespan $L E$. With that assumption the number of nymphs infected per hectare, $N_{i p}$, can be described as,

$$
N_{i_{p}}=N_{i} \cdot D
$$

Equation 8.9

In my study sites, I found an average density for Wood mice of 20 ha $^{-1}$ (Chapter 5), which would translate in $N_{i p} \approx 162 \mathrm{ha}^{-1}$ infected per cohort of Wood mice.

Using the equations above, the effect of changes in density and average daily distance travelled on the number of nymphs infected per population can be determined. This can be done by combining the different equations to express $N_{i p}$ as a function of daily distance travelled and density as,

$$
N_{i_{p}}=L_{i} \cdot s \cdot D=\left(\sum_{t=\frac{1}{\delta_{l}}}^{L E} \alpha_{l} \cdot M \cdot\left(1-\left(1-\left(\alpha_{n} \cdot \iota_{n} \cdot M\right)\right)^{t}\right)\right) \cdot s \cdot D \quad \text { Equation } 8.10
$$




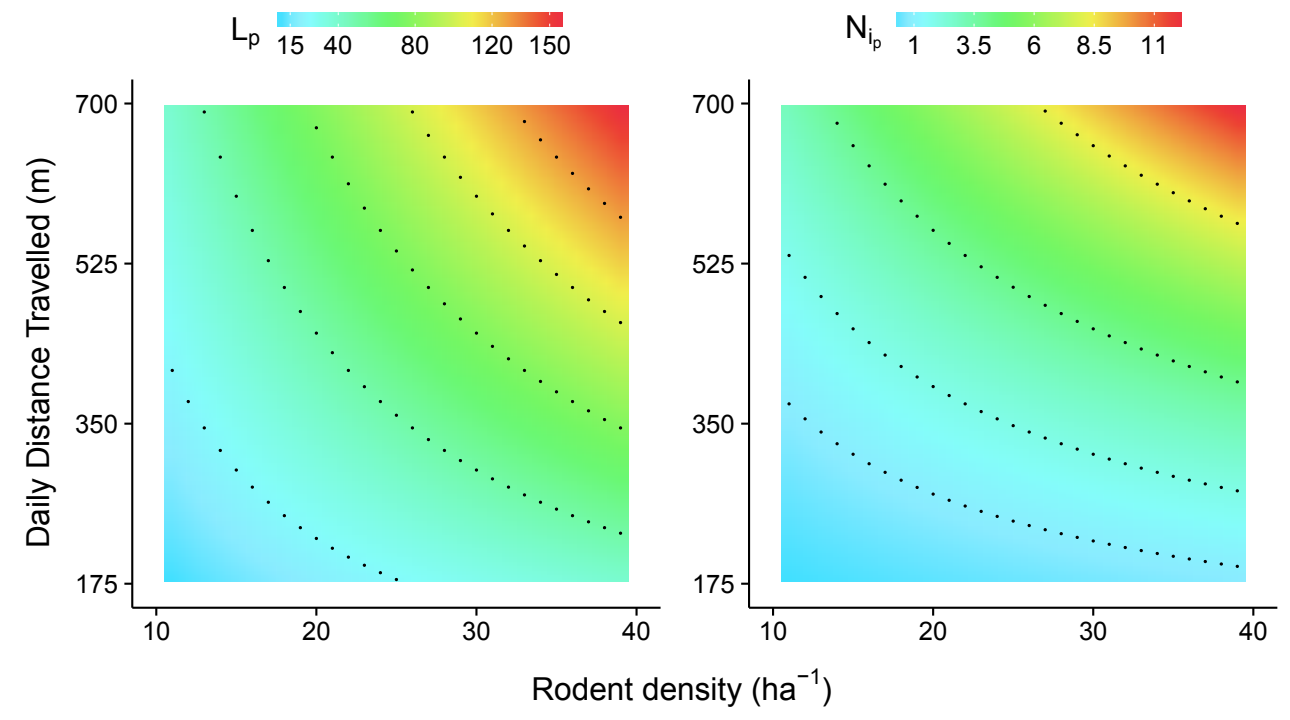

Figure 8.3. Model outcome for number of larvae fed, $L_{p}\left(100 \mathrm{~m}^{-2}\right)$, and number of nymphs infected with $B$. afzelii, $N_{i p}\left(100 \mathrm{~m}^{-2}\right)$, by populations of Wood mice with varying density and daily distance travelled. Dotted lines show combinations of density and daily distance travelled that result in the same number of (infected) ticks.

From equation 8.10 it is clear that changing daily distance travelled, $M$, has a larger impact on $N_{i p}$ than changing host density, $D$. This can also be seen in the output of a simple model calculating the number of nymphs infected with $B$. afzelii by populations of Wood mice with varying densities and daily distances travelled per individual mouse, where a twofold increase in $D$ leads to a twofold increase in $N_{i p}$, while a twofold increase in $M$ results in almost a fourfold increase in $N_{i p}$ (Figure 8.3).

\section{Validation of model assumptions}

From the model it is clear that, under the given assumptions (Box 8.1), changes in daily distance travelled by reservoir-competent host individuals have a larger effect on the number of ticks infected by a population than changes in host density. Below, I discuss the different assumptions and possible implications of deviations from these assumptions.

In the model, I assumed that both larval and nymphal densities are independent of host density. Due to the multi-year life cycle of Ixodes ticks, there is a time lag on the effect of host density on tick density and infection prevalence in questing ticks $^{8}$. Therefore, it can be stated that at any one moment in time, tick density 


\section{Box 8.1.}

Assumptions underlying the presented model on the influence of daily distance travelled and host density on tick-borne pathogen dynamics.

1. Both larval and nymphal densities are independent of host density.

2. The probability of encountering a larva is independent on the probability of encountering a nymph.

3. The number of larvae and nymphs encountered is linearly correlated to the daily distance travelled with slope = encounter rate.

4. Larval and nymphal encounter rate $\left(\mathrm{m}^{-1}\right)$ is independent of host movement.

5. Hosts get infected only by the nymphal stage.

6. Nymphal infection prevalence is independent of host movement or density.

7. The availability of larvae and nymphs is independent on the number of larvae and nymphs that have already found a host.

8. Once an infected nymphs is encountered, the host becomes infected instantaneously and can directly transmit the infection to encountered larvae.

9. Infected hosts infect encountered larvae for the rest of their lifespan.

10. Realized reservoir competence is proportional to the infection rate in hosts.

11. All parameters that influence the survival of feeding and engorged ticks (resistance/tolerance/grooming) are similar for all host individuals.

12. Daily distance travelled is independent of host density.

and infection prevalence are not dependent on the host density at that specific time, in which case assumptions 1 and 6 (Box 8.1) will hold for any specific time step. When this model is used to study temporal changes in tick-borne pathogen prevalence, these factors should however be taken into account.

I assumed that the encounter rate for larvae, $\alpha$, was independent of the encounter rate for nymphs, $\alpha_{n}$. It was difficult to test this assumption, as there are no empirical studies estimating the encounter rate between ticks and hosts from the host perspective. There are studies that estimate host contact rate from the tick perspective but these only consider host density as a factor determining 


\section{8 | Chapter 8}

contact rate ${ }^{280}$. In my study sites (Chapter 4-7) the number of larvae counted per $10 \mathrm{~m}$ drag was positively correlated with the number of nymphs counted on the same drag (generalized linear mixed model with a negative binomial distribution and a random intercept per month nested in site: $\beta=0.2, p<0.001$ ), suggesting that $\alpha_{1}$ and $\alpha_{n}$ could be correlated as well. There was a very large variation in larval and nymphal counts between drags, indicating that larval and nymphal densities were clustered. This clustered spatial distribution of ticks could also mean that the number of ticks encountered is not linear with daily distance travelled (Box 8.1; assumption 3). Moving $1 \mathrm{~m}$ in a patch with very high tick density will result in another number of ticks encountered than moving $1 \mathrm{~m}$ in a patch with low tick density. Both these effects will influence the outcome of the model in an absolute sense, but should not affect the relative contribution of daily distance travelled and density. Furthermore, they show that the spatial distribution of ticks and the spatial movement of hosts should be made explicit in models of tick-borne pathogen prevalence. This could be done in an individual-based model where the effect of spatial aggregation of ticks and spatial behaviour of hosts could be modelled explicitly.

My model is based on the assumption that the number of ticks encountered per distance moved is independent of host movement (Box 8.1; assumption 4). Animal movement is determined by several factors, including the size of the animal, the availability of resources and the presence of predators and competitors ${ }^{281,282}$ (for further discussion see below). As tick burden most probably has a very low fitness $\operatorname{cost}^{283}$, it is unlikely that hosts change their movement as a response to tick presence (but see Allan et al. ${ }^{284}$ ). Therefore, the assumption that tick encounter is independent of host movement seems reasonable. However, the speed with which animals move might affect encounter rate. Animals that move over a larger distance per day can do so by increasing the speed with which they move, or by increasing the time spent active ${ }^{186}$. There is however, to my knowledge, no information on the relationship between movement speed and the chance that an encountered tick will attach to the host. Spatially explicit models could be used to test the effect of deviations from this assumption on model outcome, where the number of ticks encountered per day could be modelled as a function of movement pattern, speed, time spent active and a speed-determined tickencounter rate.

I assumed that hosts only get infected by feeding an infected nymph. This assumption only holds for certain pathogens that are not trans-ovarially 
transmitted ${ }^{22}$ and for host species that do not feed adult ticks. However, even pathogens that are considered not to be trans-ovarially transmitted, such as $B$. afzelii, can still be found in very low prevalence in questing larvae, which can therefore have a major contribution to infection in rodents ${ }^{256}$. Incorporating infection by larvae into the model would further increase the importance of daily distance travelled as an important determinant of infection prevalence in hosts and subsequently in infected nymphs.

The assumption that the number of ticks encountered is independent of the number of ticks that already found a host is controversial. Most models considering the effects of host diversity and abundance on pathogen prevalence in ticks have assumed that tick numbers are limited and are distributed over the hosts according to the abundance of these hosts $20,27,33,285,286$. However, the low survival rates between the different life stages of $I$. ricinus imply that the majority of larvae and nymphs do not survive to become adults ${ }^{22}$. Indeed, the few studies that have tried to estimate the chance that a tick finds a host estimated this chance to be very low ${ }^{22,280}$. This suggests that the majority of larvae and nymphs never find a host, which would mean that every added host will just feed the ticks that otherwise would not have found a host ${ }^{158}$. This implies that, at least for larvae and nymphs, the availability of ticks in the vegetation is not limiting and the assumption holds.

I assumed that hosts are immediately infective after encountering an infected nymph and that larvae are immediately infected when encountering a host (Box 8.1; assumptions 8). However, infected ticks encountering a host do not immediately infect the host and feeding ticks do not immediately get infected ${ }^{287}$. Furthermore, hosts have a latent period between getting infected and being infective to feeding ticks which is different for each pathogen. For example, B. burgdorferi s.l. in rodents has a latent period of 7 days ${ }^{22}$. Incorporating these time lags would affect the absolute number of nymphs infected by each individual rodent, but would not affect the relative contribution of daily distance travelled and host density and therefore does not impact the implications of the model. While many studies incorporate host recovery after infection for different pathogens ${ }^{269}$, empirical studies on B. burgdorferi s.l. show that rodents do not recover from infection and stay infectious to feeding ticks for the rest of their lifespan 22,288 (Box 8.1; assumption 9).

For B. burgdorferi s.l., infection prevalence and realized reservoir competence are strongly correlated (Chapter 2; Box 8.1; assumption 10). Kurtenbach et al. ${ }^{24}$ 


\section{0 | Chapter 8}

showed that with experimental infection in the lab there are differences in reservoir competence between host species and Dizij and Kurtenbach ${ }^{23}$ showed that host species differ in their resistance against ticks (Box 8.1; assumption 11). As these differences were linked to differences in immuno-competence, it is probable that individuals also differ in their reservoir competence. Furthermore, grooming behaviour differs between species and individuals ${ }^{20,289}$. These differences could be taken into account in the model by including reservoir competence as an extra parameter into the estimation of $L_{i}$ in equation 8.7 and by including a parameter for the percentage of ticks surviving resistance or grooming in equation 8.5. This would, however, only influence the absolute number of larvae fed and number of nymphs infected and not the relative contribution of daily distance travelled and density of reservoir-competent hosts.

I assumed that daily distance travelled and host density are independent. However, this is unlikely as spatial behaviour of animals is often related to home range size, and home range size is related to density. Radio-tracked Wood mice showed a decrease in daily distance travelled with increasing densities ${ }^{213}$. Similarly, network analyses showed that Field voles (Microtus agrestis) moved less and were more spatially constrained in their contacts at high population densities ${ }^{242}$. These studies suggest that rodents have smaller home ranges at high population densities, which will lead to lower daily distances travelled. This implies that it is very difficult to distinguish effects of daily distance travelled and true density effects when doing an empirical study and both factors should be taken into account to better understand the role of both on tick burden and tickborne pathogen dynamics.

The impact of host behaviour on tick-borne pathogen dynamics Although none of the assumptions will hold for all systems, there are some systems and situations in which the assumptions made by the model I presented here will be met. Many assumptions will mainly affect the absolute outcome of the model and not the relative contribution of daily distance travelled and density. Under the given assumptions, I showed that for a system with Wood mice and $B$. afzelii, daily distance travelled by Wood mice has a larger impact on the density of infected nymphs than the density of Wood mice. This is in agreement with the finding that the tick burden on rodents, but not rodent density was correlated with the density of nymphs infected with tick-borne pathogens transmitted by those rodents (Chapters $6 \& 7$ ). 
An increase in daily distance travelled will increase the average burden of both larvae and nymphs, which will impact tick-borne pathogens that are transmitted by co-feeding transmission ${ }^{290}$. Tick-borne encephalitis virus is the most important tick-borne pathogen that is mainly transmitted by co-feeding transmission ${ }^{291}$. Therefore, the spatial behaviour of hosts should be taken into account in models investigating tick-borne encephalitis virus transmission. Similarly, co-infections with multiple pathogens can have profound effects on pathogen transmission ${ }^{292}$. Animals that walk more not only have a larger probability of becoming infected with one pathogen, but they also have a larger probability of becoming infected with multiple pathogens, further increasing the importance of spatial behaviour on tick-borne pathogen prevalence.

The role of spatial behaviour might be less important for tick-borne pathogens that are maintained by a high infection prevalence in hosts compared to pathogens that are maintained by a high infection prevalence in questing ticks ${ }^{190}$ (Chapter 2). For example, using nymphal burden on Blackbirds as determined in Chapter 2, infection prevalence of $B$. garinii in nymphs as found in Chapter 7 and a daily distance travelled on the ground of $200 \mathrm{~m}$ results in an infection prevalence in Blackbirds of approximately 0.84 , which is quite similar to 0.86 , the average infection prevalence of Blackbirds with B. burgdorferi s.I. ${ }^{190}$ (Chapter 2). Halving the distance travelled daily to $100 \mathrm{~m}$ results in an infection prevalence of 0.60 , which is a much smaller reduction than was found for a similar reduction in travel distance for the Wood mouse model. Nevertheless, a reduction in spatial behaviour still results in a decrease in infection prevalence and therefore daily distance travelled has a larger effect on the density of infected nymphs than host density.

From these examples I conclude that the spatial behaviour of reservoircompetent hosts should be taken into account as an important determinant of tick burden and tick-borne pathogen dynamics.

\section{Integrating behavioural ecology into disease ecology}

Keesing et al. ${ }^{31}$ theorized that the presence of other species (competitors and predators) could influence disease transmission both for directly transmitted pathogens and vector-borne pathogens by affecting the rate of contact between infected and non-infected host individuals, between infected hosts and noninfected vectors and between infected vectors and non-infected hosts (encounter 


\section{2 | Chapter 8}

reduction/augmentation). There is some empirical evidence that this might be the case. For example, Khalil et al. ${ }^{293}$ showed that the presence of competitors and predators reduced Puumala hantavirus prevalence in Bank voles (Myodes glareolus), and I showed that the presence of predators and competitors can reduce the tick burden on rodents, resulting in a reduction of tick-borne disease risk (Chapter 5-6). Although these studies did not investigate how predators and competitors changed vector and pathogen prevalence, both speculated that the behaviour of hosts might play a large role. To my knowledge, there is only one study that specifically took behavioural changes of host species as a response to predation risk into account as a determinant of vector-borne disease dynamics ${ }^{294}$.

Above, I showed that the spatial behaviour of hosts plays an important role in determining encounter rates between individual hosts and ticks. A substantial amount of work has been done on the factors driving and determining movement of animals ${ }^{295}$. I will limit my discussion to effects of predators and competitors on the spatial behaviour of vertebrate species, as these effects might change disease transmission as a response to changes in the vertebrate assemblage.

\section{Predator and competitor induced changes in animal behaviour}

Behavioural ecologists have studied the behavioural responses of prey to predators or cues of predators both theoretically and empirically. Theoretically, prey face a trade-off between finding enough food and reducing predation risk ${ }^{296}$. This should result in a reduction of activity as a response to predators in areas with sufficient food. Indeed, empirical studies have shown that prey can both reduce their activity or change their habitat use to 'safer' habitats in response to the presence of predators or perceived predation risk ${ }^{297}$. For example, Field voles decreased their activity and day range in response to the presence of Weasels (Mustela nivalis) and Stoats (M. erminea) $)^{214}$, and Bank voles decreased their activity as a response to the presence of Weasel scent ${ }^{229}$. Also larger host species, such as Red deer (Cervus elaphus) change their foraging behaviour and habitat use in response to predators ${ }^{298}$. Birds can respond to predators by changing their behaviour in three dimensions. For example, Blackbirds decreased the time spent on the ground as a response to predators ${ }^{299}$. The response of prey to predators can even have consequences on a population level, as predator induced movement can result in changes in local densities ${ }^{300}$.

Apart from predators, the presence of competitors can also change animal behaviour. The presence of conspecifics or heterospecifics can both reduce home 


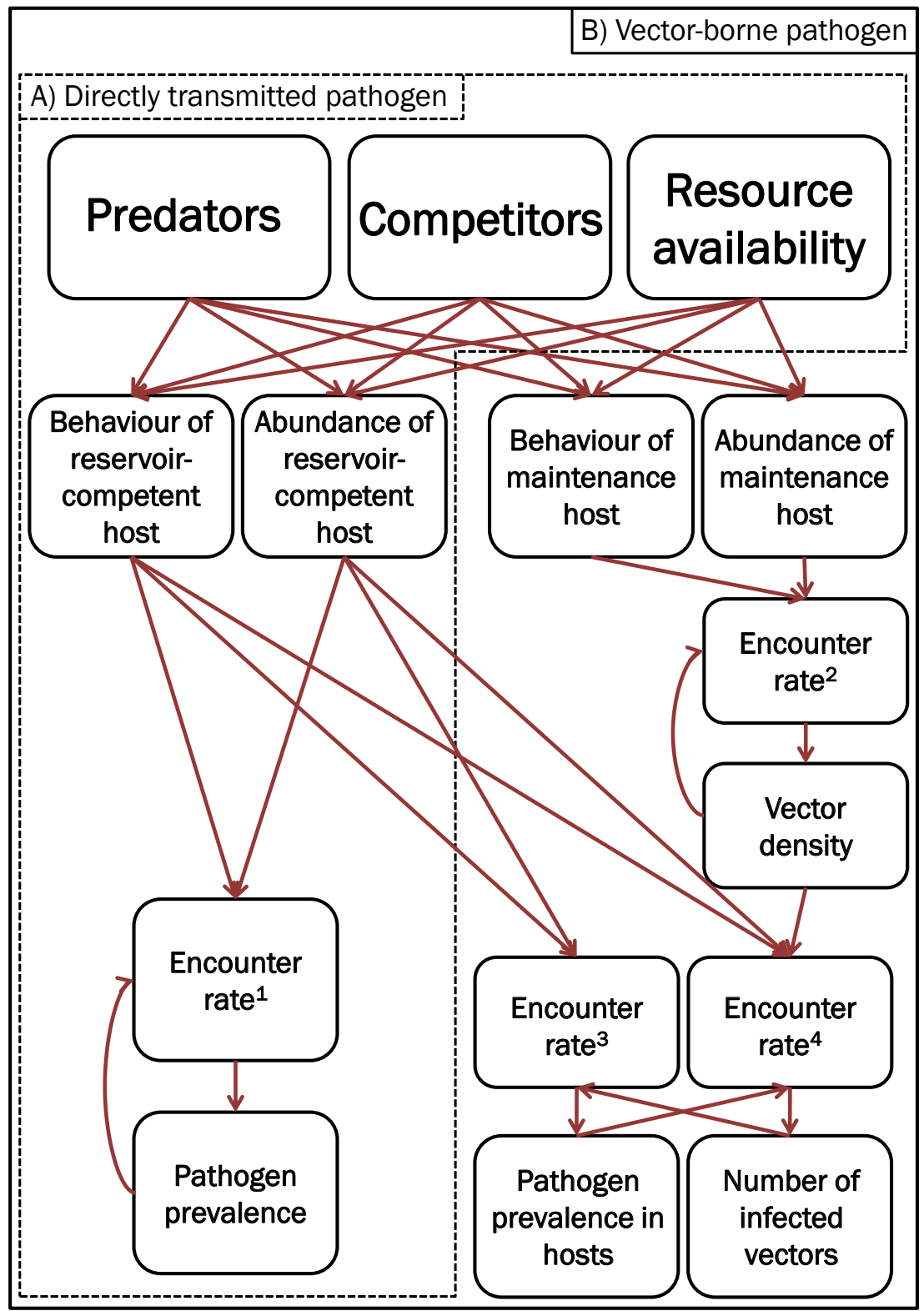

Figure 8.4. Theoretical framework showing how the presence of predators, competitors and resources can influence both A) directly transmitted and B) vector-borne pathogen prevalence, via changes in behaviour and abundance of both reservoir competent and maintenance hosts. ${ }^{1}$ encounter rate between susceptible and infected reservoir-competent host individuals, ${ }^{2}$ encounter rate between maintenance hosts and vectors, ${ }^{3}$ encounter rate between uninfected reservoir-competent hosts and infected vectors, and ${ }^{4}$ encounter rate between infected reservoir competent hosts and uninfected vectors. 


\section{4 | Chapter 8}

range size ${ }^{213,301}$. For example, Bank voles changed their home range size and activity as a response to the presence of Common shrews (Sorex araneus) ${ }^{302}$. Depending on the situation, the presence of competitors can be as important determining home range size as the availability of food ${ }^{303}$. Animals with a smaller home range often also move over smaller distances per day ${ }^{213}$. Therefore, the presence of predators or competitors can result in changes in daily distance travelled of reservoir-competent hosts, thus changing the encounter rate between these hosts and pathogens.

\section{Behavioural disease ecology}

The examples shown above indicate that changes in vertebrate assemblages can have profound effects on the spatial behaviour of animals. I also showed that, at least theoretically, daily distance travelled has a large effect on the encounter rate of reservoir-competent hosts with ticks. Therefore, existing models investigating the effect of changes in host assemblage, such as the models by Keesing et al. ${ }^{20}$ and Levi et al. ${ }^{241}$, are incomplete and ignore important interactions between host species that will change the dynamics of tick-borne pathogens. Also models investigating the effect of predators on disease risk often only incorporate direct effects of predators ${ }^{304,305}$, while it is clear that predators also have many indirect effects on prey which might affect pathogen dynamics. Furthermore, (spatial) behaviour of animals is influenced by many other factors, such as the size of the animal and resource availability ${ }^{67,282}$. Therefore, disease ecology, and in particular the study of vector-borne pathogens, could benefit from incorporating the fast amount of theory provided by the fields of behavioural and movement ecology to better understand how spatial behaviour of animals affects pathogen dynamics at different spatial scales. This could be done by integrating models and theory from the different fields into a new discipline: behavioural disease ecology. For example, parasites could be added to predator-prey game models ${ }^{306}$ to study the effect of behavioural changes of predators and prey on parasite distribution on small spatial scales, or spatially explicit models might be used to determine the relative importance of direct and indirect transmission for pathogens such as Puumala hantavirus ${ }^{307}$.

For directly transmitted pathogens the presence of predators and competitors and resource availability will influence both the spatial behaviour and abundance of reservoir-competent hosts, which will affect the encounter rate between susceptible and infected individuals (Figure 8.4). The impact will, however, be 
much larger for vector-borne pathogens, as the change in behaviour of reservoircompetent hosts will affect both the encounter rate of uninfected hosts with infected vectors and the encounter rate of infected hosts with uninfected vectors. Furthermore, the presence of predators and competitors and resource availability will also influence the behaviour and abundance of maintenance hosts for the vector, which will affect encounter rate between vector and maintenance host, and therefore vector density (Figure 8.4).

The integration of spatial behaviour of hosts into models of pathogen maintenance and transmission will lead to better understanding of the spatial epidemiology of pathogens ${ }^{308}$ both on large and small spatial scales. By better understanding the mechanisms behind pathogen transmission in assemblages of hosts, ecologists and public health officials will be able to understand and perhaps even forecast how changes in vertebrate assemblages will influence pathogen prevalence. This is needed as an increasing human population and continuous changes to the environment have led to a world in which animals have to adapt to live closer to humans, increasing the disease risk for zoonotic diseases $^{309}$.

\section{Tick-borne disease dynamics in the Anthropocene}

Ixodes ricinus populations have expanded and increased in density over the last couple of decades ${ }^{61}$. This can partly be explained by an increase in population size of the most important host species (Bank vole, Blackbird, Red deer, Roe deer, and Wood mouse) in Europe ${ }^{147-149}$. These species have most probably been so successful because they have been able to adapt to a fragmented and humandominated landscape ${ }^{310,311}$. In the future, vertebrate assemblages are expected to further change as both small to medium-sized ${ }^{312}$ and large ${ }^{313}$ carnivores have also started to increase their distribution in Europe.

The increase in carnivores and the return of apex predators to some parts of Europe $^{313}$ might result in cascading effects on tick-borne disease risk by changes in prey behaviour (see above). In Chapter $5 \& 6$ I showed that the tick burden on rodents decreases with the relative abundance of medium-sized (meso) predators. The comeback of apex predators, such as the Wolf (Canis lupus) and Eurasian lynx (Lynx lynx), can have a regulating effect on these mesopredators ${ }^{314}$, which might result in cascading effects on tick-borne disease risk ${ }^{247}$. In contrast, the presence of apex predators will change behaviour and densities of deer ${ }^{315,316}$, 


\section{6 | Chapter 8}

which might impact tick-borne disease risk more directly. Therefore, the current comeback of carnivores in Europe will change the behaviour and densities of host species, and might thus indirectly change tick-borne disease risk.

Another change in distribution and behaviour of vertebrate hosts for ticks in Europe is the adaptation of species to living in urban areas. More and more bird species have adapted to living in an urbanized world ${ }^{317}$, and rodents have been thriving close to humans for centuries ${ }^{318}$. Although deer might not be present in the centre of urbanized areas, Roe deer have formed small populations in city parks ${ }^{319}$. In the current situation, tick populations in the city seem to be relatively low, and Blackbirds living in urban areas have lower tick burden than Blackbirds living in the forest ${ }^{320}$. However, with increasing deer densities in the city as a possible response to the comeback of apex predators, this might change. Furthermore, by moving into the city, ticks might come into contact with novel host species. For example, Brown rats (Rattus norvegicus), that are absent in most forests, can be an important host for ticks in urban environments ${ }^{321}$. This spillover into new host species is very hard to predict and might result in novel host species with a very high reservoir competence ${ }^{322}$.

Within cities, deer might be present in city parks, while hedgehogs might take over the role of deer as maintenance hosts in gardens ${ }^{75}$. The release from predation has been suggested as the driver of increased hedgehog abundances in urban areas ${ }^{323,324}$. Both the European hedgehog (Erinaceus europaeus) in Western Europe and the Northern white-breasted hedgehog (E. roumanicus) in Eastern Europe are reservoirs for different genospecies of B. burgdorferi s.l. including one of the causative agents of neuroborreliosis, B. bavariensis ${ }^{325,326}$. Also Blackbirds, an important host species for another neuroborreliosis causing genospecies, $B$. garinii, is present in high densities in urban areas, especially in gardens ${ }^{327}$. Generally, the few species that survive in urban areas occur in high densities $^{328}$, which suggests that reservoir-competent species can reach high densities in urban areas. Due to these high densities, the infection prevalence in ticks in cities and city parks might be very high as was found in a city park in Munich ${ }^{329}$. The high amount of time people spent in city parks and gardens further increases the risk of getting Lyme borreliosis in these habitats ${ }^{330,331}$. As a result, tick-borne disease risk in urban areas might already be higher than expected, and will most probably increase in the near future. Therefore, studies in urban areas are needed to better understand how urbanization, adaptation of vertebrate hosts to urban areas and spillover into novel host species will 
influence tick-borne disease risk in urban areas.

Concluding, our world is changing and as a consequence vertebrate assemblages are also changing. This may lead to changes in I. ricinus density and infection prevalence with tick-borne pathogens. From the studies presented in this thesis I conclude that the abundance and behaviour of several host species (e.g., Bank vole, Blackbird, Red deer, Red fox, Roe deer, and Wood mouse) determines tick-borne disease risk. Therefore, studying the drivers of animal abundance and behaviour related to ticks and pathogens will be the next step in better understanding and describing tick-borne disease risk. The ecology of tickborne pathogens is very complex and targeting vertebrate hosts for intervention strategies will be both inefficient and costly due to the intricate interplay between multiple vertebrate host species. Therefore, I conclude that prevention of tick bites is the best way to reduce tick-borne disease incidence. 
168 


\section{BIBLIOGRAPHY}

1 Taylor, L. H., Latham, S. M. \& Woolhouse, M. E. J. Risk factors for human disease emergence. Philosophical Transactions of the Royal Society of London Series B-Biological Sciences 356, 983-989 (2001).

2 Jones, K. E. et al. Global trends in emerging infectious diseases. Nature 451, 990-993, doi:10.1038/ nature06536 (2008).

3 Ostfeld, R. S. \& Keesing, F. The function of biodiversity in the ecology of vector-borne zoonotic diseases. Canadian Journal of Zoology-Revue Canadienne De Zoologie 78, 2061-2078, doi:10.1139/cjz-78-122061 (2000).

4 Woolhouse, M. E. J., Taylor, L. H. \& Haydon, D. T. Population biology of multihost pathogens. Science 292, 1109-1112, doi:10.1126/science.1059026 (2001).

5 Holdenried, R., Evans, F. C. \& Longanecker, D. S. Host-Parasite-Disease Relationships in a Mammalian Commuinty in the Central Coast Range of California. Ecol. Monogr. 21, 1-18, doi:10.2307/1948643 (1951).

6 Samia, N. I. et al. Dynamics of the plague-wildlife-human system in Central Asia are controlled by two epidemiological thresholds. Proc. Natl. Acad. Sci. USA 108, 14527-14532, doi:10.1073/pnas.1015946108 (2011).

7 Cardon, M., Loot, G., Grenouillet, G. \& Blanchet, S. Host characteristics and environmental factors differentially drive the burden and pathogenicity of an ectoparasite: A multilevel causal analysis. J. Anim. Ecol. 80, 657-667, doi:10.1111/j.1365-2656.2011.01804.x (2011).

8 Randolph, S. E. Tick ecology: processes and patterns behind the epidemiological risk posed by ixodid ticks as vectors. Parasitology 129, S37-S65, doi:10.1017/s0031182004004925 (2004).

9 Välimäki, P. et al. Geographical variation in host use of a blood-feeding ectoparasitic fly: Implications for population invasiveness. Oecologia 166, 985-995, doi:10.1007/s00442-011-1951-y (2011).

10 Estrada-Pena, A. The relationships between habitat topology, critical scales of connectivity and tick abundance Ixodes ricinus in a heterogeneous landscape in northern Spain. Ecography 26, 661-671, doi:10.1034/j.1600-0587.2003.03530.x (2003).

11 Lopez, J. E., Gallinot, L. P. \& Wade, M. J. Spread of parasites in metapopulations: An experimental study of the effects of host migration rate and local host population size. Parasitology 130, 323-332, doi:10.1017/s0031182004006602 (2005).

12 Calabrese, J. M., Brunner, J. L. \& Ostfeld, R. S. Partitioning the Aggregation of Parasites on Hosts into Intrinsic and Extrinsic Components via an Extended Poisson-Gamma Mixture Model. PLoS One 6, e29215, doi:10.1371/journal.pone.0029215 (2011). 
13 Jackson, J. A. et al. An Immunological Marker of Tolerance to Infection in Wild Rodents. PLoS Biol. 12, e1001901 (2014).

14 Krasnov, B., Khokhlova, I. \& Shenbrot, G. The effect of host density on ectoparasite distribution: An example of a rodent parasitized by fleas. Ecology 83, 164-175 (2002).

15 Pfäffle, M., Petney, T., Skuballa, J. \& Taraschewski, H. Comparative population dynamics of a generalist (Ixodes ricinus) and specialist tick (I. hexagonus) species from European hedgehogs. Exp. Appl. Acarol. 54, 151-164, doi:10.1007/s10493-011-9432-x (2011).

16 Streicker, D. G., Fenton, A. \& Pedersen, A. B. Differential sources of host species heterogeneity influence the transmission and control of multihost parasites. Ecol. Lett. 16, 975-984, doi:10.1111/ele.12122 (2013).

17 Ezenwa, V. O., Price, S. A., Altizer, S., Vitone, N. D. \& Cook, K. C. Host traits and parasite species richness in even and odd-toed hoofed mammals, Artiodactyla and Perissodactyla. Oikos 115, 526-536, doi:10.1111/j.2006.0030-1299.15186.x (2006).

18 Marsot, M. et al. Which forest bird species are the main hosts of the tick, Ixodes ricinus, the vector of Borrelia burgdorferi sensu lato, during the breeding season? Int. J. Parasitol. 42, 781-788 (2012).

19 Bordes, F., Morand, S., Kelt, D. A. \& Van Vuren, D. H. Home Range and Parasite Diversity in Mammals. Am. Nat. 173, 467-474, doi:10.1086/597227 (2009).

20 Keesing, F. et al. Hosts as ecological traps for the vector of Lyme disease. Proceedings of the Royal Society B-Biological Sciences 276, 3911-3919, doi:10.1098/rspb.2009.1159 (2009).

21 LoGiudice, K., Ostfeld, R. S., Schmidt, K. A. \& Keesing, F. The ecology of infectious disease: Effects of host diversity and community composition on Lyme disease risk. Proc. Natl. Acad. Sci. USA 100, 567571, doi:10.1073/pnas.0233733100 (2003).

22 Randolph, S. E. \& Craine, N. G. General Framework for Comparative Quantitative Studies on Transmission of Tick-Borne Diseases Using Lyme Borreliosis in Europe as an Example. J. Med. Entomol. 32, 765777 (1995).

23 Dizij, A. \& Kurtenbach, K. Clethrionomys glareolus, but not Apodemus flavicollis, acquires resistance to Ixodes ricinus L., the main European vector of Borrelia burgdorferi. Parasite Immunol. 17, 177-183, doi:10.1111/j.1365-3024.1995.tb00887.x (1995).

24 Kurtenbach, K. et al. Differential immune responses to Borrelia burgdorferi in European wild rodent species influence spirochete transmission to Ixodes ricinus L. (Acari: Ixodidae). Infect. Immun. 62, 53445352 (1994).

25 Brunner, J. L., LoGiudice, K. \& Ostfeld, R. S. Estimating reservoir competence of Borrelia burgdorferi hosts: Prevalence and infectivity, sensitivity, and specificity. J. Med. Entomol. 45, 139-147, doi:10.1603 /0022-2585(2008)45[139:ercobb]2.0.co;2 (2008).

26 Kilpatrick, A. M., Daszak, P., Jones, M. J., Marra, P. P. \& Kramer, L. D. Host heterogeneity dominates West Nile virus transmission. Proceedings of the Royal Society B: Biological Sciences 273, 2327-2333, doi:10.1098/rspb.2006.3575 (2006).

27 Van Buskirk, J. \& Ostfeld, R. S. Controlling Lyme Disease by Modifying the Density and Species Composition of Tick Hosts. Ecol. Appl. 5, 1133-1140 (1995).

28 Hamer, G. L. et al. Host selection by Culex pipiens mosquitoes and west nile virus amplification. Am. J. Trop. Med. Hyg. 80, 268-278 (2009).

29 Kawecki, T. J. Red queen meets Santa Rosalia: Arms races and the evolution of host specialization in organisms with parasitic lifestyles. Am. Nat. 152, 635-651, doi:10.1086/286195 (1998). 
30 Ostfeld, R. S. et al. Life History and Demographic Drivers of Reservoir Competence for Three Tick-Borne Zoonotic Pathogens. PLoS One 9, e107387 (2014).

31 Keesing, F., Holt, R. D. \& Ostfeld, R. S. Effects of species diversity on disease risk. Ecol. Lett. 9, 485-498, doi:10.1111/j.1461-0248.2006.00885.x (2006).

32 Cagnacci, F. et al. Effects of deer density on tick infestation of rodents and the hazard of tick-borne encephalitis. I: Empirical assessment. Int. J. Parasitol. 42, 365-372 (2012).

33 Gilbert, L., Norman, R., Laurenson, K. M., Reid, H. W. \& Hudson, P. J. Disease persistence and apparent competition in a three-host community: an empirical and analytical study of large-scale, wild populations. J. Anim. Ecol. 70, 1053-1061, doi:10.1046/j.0021-8790.2001.00558.x (2001).

34 Johnson, P. T. J., Ostfeld, R. S. \& Keesing, F. Frontiers in research on biodiversity and disease. Ecol. Lett. 18, 1119-1133, doi:10.1111/ele.12479 (2015).

35 Piesman, J. \& Eisen, L. in Annu. Rev. Entomol. Vol. 53 Annual Review of Entomology 323-343 (Annual Reviews, 2008).

36 Gray, J. S. The ecology of ticks transmitting Lyme borreliosis. Exp. Appl. Acarol. 22, 249-258, doi:10.1023/a:1006070416135 (1998).

37 Jaenson, T. G. T. et al. Geographical Distribution, Host Associations, and Vector Roles of Ticks (Acari: Ixodidae, Argasidae) in Sweden. J. Med. Entomol. 31, 240-256 (1994).

38 Gilbert, L., Maffey, G. L., Ramsay, S. L. \& Hester, A. J. The effect of deer Management on the abundance of Ixodes ricinus in Scotland. Ecol. Appl. 22, 658-667 (2012).

39 Sprong, H. et al. Circumstantial evidence for an increase in the total number and activity of borrelia-infected ixodes ricinus in the Netherlands. Parasit. Vectors 5, 294, doi:10.1186/1756-3305-5-294 (2012).

40 Mejlon, H. A. \& Jaenson, T. G. T. Questing behaviour of Ixodes ricinus ticks (Acari: Ixodidae). Exp. Appl. Acarol. 21, 747-754, doi:10.1023/a:1018421105231 (1997).

41 Rauter, C. \& Hartung, T. Prevalence of Borrelia burgdorferi sensu lato genospecies in Ixodes ricinus ticks in Europe: a metaanalysis. Appl. Environ. Microbiol. 71, 7203-7216, doi:10.1128/aem.71.11.72037216.2005 (2005).

42 Stuen, S., Granquist, E. G. \& Silaghi, C. Anaplasma phagocytophilum - a widespread multi-host pathogen with highly adaptive strategies. Frontiers in Cellular and Infection Microbiology 3, 31, doi:10.3389/ fcimb.2013.00031 (2013).

43 Wagemakers, A., Staarink, P. J., Sprong, H. \& Hovius, J. W. R. Borrelia miyamotoi: a widespread tickborne relapsing fever spirochete. Trends Parasitol. 31, 260-269, doi:http://dx.doi.org/10.1016/j. pt.2015.03.008 (2015).

44 Silaghi, C., Beck, R., Oteo, J. A., Pfeffer, M. \& Sprong, H. Neoehrlichiosis: an emerging tick-borne zoonosis caused by Candidatus Neoehrlichia mikurensis. Exp. Appl. Acarol. 68, 279-297, doi:10.1007/s10493015-9935-y (2015).

45 Stanek, G., Wormser, G. P., Gray, J. \& Strle, F. Lyme borreliosis. Lancet 379, 461-473, doi:10.1016/ s0140-6736(11)60103-7 (2012).

46 Margos, G., Vollmer, S. A., Ogden, N. H. \& Fish, D. Population genetics, taxonomy, phylogeny and evolution of Borrelia burgdorferi sensu lato. Infection Genetics and Evolution 11, 1545-1563, doi:10.1016/j. meegid.2011.07.022 (2011).

47 Humair, P. F. \& Gern, L. The wild hidden face of Lyme borreliosis in Europe. Microb. Infect. 2, 915-922, doi:10.1016/s1286-4579(00)00393-2 (2000). 


\section{2 | Bibliography}

48 Kurtenbach, K. et al. Host association of Borrelia burgdorferi sensu lato - the key role of host complement. Trends Microbiol. 10, 74-79, doi:10.1016/s0966-842x(01)02298-3 (2002).

49 Kurtenbach, K. et al. Fundamental processes in the evolutionary ecology of Lyme borreliosis. Nature Reviews Microbiology 4, 660-669, doi:10.1038/nrmicro1475 (2006).

50 Burri, C., Schumann, O., Schumann, C. \& Gern, L. Are Apodemus spp. mice and Myodes glareolus reservoirs for Borrelia miyamotoi, Candidatus Neoehrlichia mikurensis, Rickettsia helvetica, R. monacensis and Anaplasma phagocytophilum? Ticks Tick Borne Dis. 5, 245-251, doi:http://dx.doi.org/10.1016/j. ttbdis.2013.11.007 (2014).

51 Jahfari, S. et al. Circulation of four Anaplasma phagocytophilum ecotypes in Europe. Parasit. Vectors 7, 365 (2014).

52 Rowcliffe, J. M. \& Carbone, C. Surveys using camera traps: are we looking to a brighter future? Anim. Conserv. 11, 185-186, doi:10.1111/j.1469-1795.2008.00180.x (2008).

53 Welbourne, D. J., Claridge, A. W., Paull, D. J. \& Lambert, A. How do passive infrared triggered camera traps operate and why does it matter? Breaking down common misconceptions. Remote Sensing in Ecology and Conservation 2, 77-83, doi:10.1002/rse2.20 (2016).

54 Rowcliffe, J. M., Carbone, C., Jansen, P. A., Kays, R. \& Kranstauber, B. Quantifying the sensitivity of camera traps: an adapted distance sampling approach. Methods in Ecology and Evolution 2, 464-476, doi:10.1111/j.2041-210X.2011.00094.x (2011).

55 Jensen, P. M., Hansen, H. \& Frandsen, F. Spatial risk assessment for lyme borreliosis Denmark. Scand. J. Infect. Dis. 32, 545-550 (2000).

56 Sykes, R. A. \& Makiello, P. An estimate of Lyme borreliosis incidence in Western Europe. Journal of Public Health Online first, doi:10.1093/pubmed/fdw017 (2016).

57 Jongejan, F. \& Uilenberg, G. The global importance of ticks. Parasitology 129, S3-S14, doi:10.1017/ s0031182004005967 (2004).

58 Baranton, G. et al. Delineation of Borrelia burgdorferi Sensu Stricto, Borrelia garinii sp. nov., and Group VS461 Associated with Lyme Borreliosis. Int. J. Syst. Bacteriol. 42, 378-383 (1992).

59 Xu, G., Fang, Q. Q., Keirans, J. E. \& Durden, L. A. Molecular Phylogenetic Analyses Indicate that the Ixodes ricinus Complex Is a Paraphyletic Group. J. Parasitol. 89, 452-457, doi:10.2307/3286032 (2003).

60 Dantas-Torres, F., Chomel, B. B. \& Otranto, D. Ticks and tick-borne diseases: A One Health perspective. Trends Parasitol. 28, 437-446, doi:10.1016/j.pt.2012.07.003 C2 - 22902521 (2012).

61 Medlock, J. M. et al. Driving forces for changes in geographical distribution of Ixodes ricinus ticks in Europe. Parasit. Vectors 6, 1, doi:10.1186/1756-3305-6-1 (2013).

62 Mannelli, A., Bertolotti, L., Gern, L. \& Gray, J. Ecology of Borrelia burgdorferi sensu lato in Europe: Transmission dynamics in multi-host systems, influence of molecular processes and effects of climate change. FEMS Microbiol. Rev. 36, 837-861 (2012).

63 Vanwambeke, S. O., Sumilo, D., Bormane, A., Lambin, E. F. \& Randolph, S. E. Landscape Predictors of Tick-Borne Encephalitis in Latvia: Land Cover, Land Use, and Land Ownership. Vector Borne Zoonotic Dis. 10, 497-506, doi:10.1089/vbz.2009.0116 (2010).

64 Tälleklint, L. \& Jaenson, T. G. T. Transmission of Borrelia burgdorferi s.l. from Mammal Reservoirs to the Primary Vector of Lyme Borreliosis, Ixodes ricinus (Acari: Ixodidae), in Sweden. J. Med. Entomol. 31, 880886 (1994).

65 Hanincova, K. et al. Association of Borrelia afzelii with rodents in Europe. Parasitology 126, 11-20, doi:10.1017/s0031182002002548 (2003). 
66 Hanincova, K. et al. Association of Borrelia garinii and B-valaisiana with songbirds in Slovakia. Appl. Environ. Microbiol. 69, 2825-2830, doi:10.1128/aem.69.5.2825-2830.2003 (2003).

67 Carbone, C., Cowlishaw, G., Isaac, N. J. B. \& Rowcliffe, J. M. How Far Do Animals Go? Determinants of Day Range in Mammals. The American Naturalist 165, 290-297, doi:10.1086/426790 (2005).

68 Lee, K. A. Linking immune defenses and life history at the levels of the individual and the species. Integr. Comp. Biol. 46, 1000-1015, doi:10.1093/icb/icl049 (2006).

69 Previtali, M. A. et al. Relationship between pace of life and immune responses in wild rodents. Oikos 121, doi:10.1111/j.1600-0706.2012.020215.x (2012).

70 Barbour, A., Bunikis, J., Fish, D. \& Hanincova, K. Association between body size and reservoir competence of mammals bearing Borrelia burgdorferi at an endemic site in the northeastern United States. Parasit. Vectors 8, 299 (2015).

71 Huang, Z. Y. X. et al. Species' Life-History Traits Explain Interspecific Variation in Reservoir Competence: A Possible Mechanism Underlying the Dilution Effect. PLoS One 8, e54341 (2013).

72 Matuschka, F. R., Fischer, P., Musgrave, K., Richter, D. \& Spielman, A. Hosts on which nymphal Ixodes ricinus most abundantly feed. Am. J. Trop. Med. Hyg. 44, 100-107 (1991).

73 Matuschka, F. R., Lange, R., Spielman, A., Richter, D. \& Fischer, P. Subadult Ixodes ricinus (Acari: Ixodidae) on Rodents in Berlin, West Germany. J. Med. Entomol. 27, 385-390 (1990).

74 Franke, J., Hildebrandt, A. \& Dorn, W. Exploring gaps in our knowledge on Lyme borreliosis spirochaetes - Updates on complex heterogeneity, ecology, and pathogenicity. Ticks Tick Borne Dis. 4, 11-25, doi:http://dx.doi.org/10.1016/j.ttbdis.2012.06.007 (2013).

75 Pfäffle, M., Littwin, N., Muders, S. V. \& Petney, T. N. The ecology of tick-borne diseases. Int. J. Parasitol. 43, 1059-1077, doi:http://dx.doi.org/10.1016/j.ijpara.2013.06.009 (2013).

76 Perkins, S. E., Cattadori, I. M., Tagliapietra, V., Rizzoli, A. P. \& Hudson, P. J. Empirical evidence for key hosts in persistence of a tick-borne disease. Int. J. Parasitol. 33, 909-917, doi:10.1016/s00207519(03)00128-0 (2003).

77 Mather, T. N., Wilson, M. L., Moore, S. I., Ribeiro, J. M. C. \& Spielman, A. Comparing the relative potential of rodents as reservoirs of the Lyme disease spirochete (Borrelia burgdorferi). Am. J. Epidemiol. 130, 143-150 (1989).

78 Kahl, O., Gern, L., Eisen, L. \& Lane, R. S. in Lyme Borreliosis: Biology, Epidemiology and Control (eds J.S. Gray, O. Kahl, R. S. Lane, \& G. Stanek) 29-46 (CABI Publishing, 2002).

79 Cramp, S. \& Perrins, C. M. Handbook of the Birds of Europe, the Middle East and North Africa. The Birds of the Western Palearctic. (Oxford University Press, 1994).

80 Niethammer, J. \& Krapp, F. Handbuch der Säugetiere Europas. (Aula-Verlag, 1978).

81 R: A language and environment for statistical computing. (R Foundation for Statistical Computing. URL http://www.R-project.org/, Vienna, Austria, 2015).

82 Burnham, K. P. \& Anderson, D. R. Multimodel inference: Understanding AIC and BIC in model selection. Sociological Methods and Research 33, 261-304, doi:10.1177/0049124104268644 (2004).

83 MuMIn: Multi-model inference. (http://CRAN.R-project.org/package=MuMIn, 2014).

84 Cook, R. D. Detection of Influential Observation in Linear Regression. Technometrics 19, 15-18, doi:10.2307/1268249 (1977).

85 Boyard, C., Vourc'h, G. \& Barnouin, J. The relationships between Ixodes ricinus and small mammal species at the woodland-pasture interface. Exp. Appl. Acarol. 44, 61-76, doi:10.1007/s10493-008-9132-3 (2008). 


\section{4 | Bibliography}

86 Brinck, P. et al. Small Mammals in Sweden as Hosts of Tick-Borne Encephalitis Virus and Vagrant Ectoparasites. Oikos 18, 124-134 (1967).

87 Cosson, J.-F. et al. Genetic characterization of the human relapsing fever spirochete Borrelia miyamotoi in vectors and animal reservoirs of Lyme disease spirochetes in France. Parasit. Vectors 7, 233 (2014).

88 Estrada-Peña, A., Osácar, J. J., Pichon, B. \& Gray, J. S. Hosts and pathogen detection for immature stages of Ixodes ricinus (Acari: Ixodidae) in North-Central Spain. Exp. Appl. Acarol. 37, 257-268, doi:10.1007/ s10493-005-3271-6 (2005).

89 Gassner, F. et al. Rodent species as natural reservoirs of Borrelia burgdorferi sensu lato in different habitats of Ixodes ricinus in The Netherlands. Ticks Tick Borne Dis. 4, 452-458, doi:http://dx.doi. org/10.1016/j.ttbdis.2012.11.017 (2013).

90 Gray, J. S., Kirstein, F., Robertson, J. N., Stein, J. \& Kahl, O. Borrelia burgdorferi sensu lato in Ixodes ricinus ticks and rodents in a recreational park in south-western Ireland. Exp. Appl. Acarol. 23, 717-729, doi:10.1023/a:1006233700194 (1999).

91 Hellgren, O., Andersson, M. \& Råberg, L. The genetic structure of Borrelia afzelii varies with geographic but not ecological sampling scale. J. Evol. Biol. 24, 159-167, doi:10.1111/j.1420-9101.2010.02148.x (2011).

92 Humair, P. F., Peter, O., Wallich, R. \& Gern, L. Strain Variation of Lyme Disease Spirochetes Isolated from Ixodes ricinus Ticks and Rodents Collected in Two Endemic Areas in Switzerland. J. Med. Entomol. 32, 433-438 (1995).

93 Humair, P. F., Rais, O. \& Gern, L. Transmission of Borrelia afzelii from Apodemus mice and Clethrionomys voles to Ixodes ricinus ticks: differential transmission pattern and overwintering maintenance. Parasitology 118, 33-42, doi:doi:null (1999).

94 Humair, P. F., Turrian, N., Aeschilimann, A. \& Gern, L. Borrelia burgdorferi in a focus of Lyme borreliosis: epizootiologic contribution of small mammals. Folia Parasitol. 40, 65-70 (1993).

95 Kiffner, C., Vor, T., Hagedorn, P., Niedrig, M. \& Ruhe, F. Factors affecting patterns of tick parasitism on forest rodents in tick-borne encephalitis risk areas, Germany. Parasitol. Res. 108, 323-335, doi:10.1007/ s00436-010-2065-x (2011).

96 Kurtenbach, K. et al. Differential transmission of the genospecies of Borrelia burgdorferi sensu lato by game birds and small rodents in England. Appl. Environ. Microbiol. 64, 1169-1174 (1998).

97 Marsot, M. et al. Introduced Siberian Chipmunks (Tamias sibiricus barberi) Contribute More to Lyme Borreliosis Risk than Native Reservoir Rodents. PLoS One 8, e55377, doi:10.1371/journal.pone.0055377 (2013).

98 Martello, E. et al. Range expansion of Ixodes ricinus to higher altitude, and co-infestation of small rodents with Dermacentor marginatus in the Northern Apennines, Italy. Ticks Tick Borne Dis. 5, 970-974, doi:10.1016/j.ttbdis.2014.07.021 (2014).

99 Matuschka, F. R., Eiffert, H., Ohlenbusch, A. \& Spielman, A. Amplifying role of edible dormice in Lyme disease transmission in central Europe. J. Infect. Dis. 170, 122-127 (1994).

100 Michalik, J., Hofman, T., Buczek, A., Skoracki, M. \& Sikora, B. Borrelia burgdorferi s.l. in Ixodes ricinus (Acari: Ixodidae) Ticks Collected from Vegetation and Small Rodents in Recreational Areas of the City of Poznań. J. Med. Entomol. 40, 690-697 (2003).

101 Pérez, D., Kneubühler, Y., Rais, O. \& Gern, L. Seasonality of Ixodes ricinus Ticks on Vegetation and on Rodents and Borrelia burgdorferi sensu lato Genospecies Diversity in Two Lyme Borreliosis-Endemic Areas in Switzerland. Vector Borne Zoonotic Dis. 12, 633-644, doi:10.1089/vbz.2011.0763 (2012). 
102 Råberg, L. Infection intensity and infectivity of the tick-borne pathogen Borrelia afzelii. J. Evol. Biol. 25, 1448-1453, doi:10.1111/j.1420-9101.2012.02515.x (2012).

103 Radzijevskaja, J. et al. The propensity of voles and mice to transmit Borrelia burgdorferi sensu lato infection to feeding ticks. Vet. Parasitol. 197, 318-325, doi:10.1016/j.vetpar.2013.06.008 (2013).

104 Richter, D. \& Matuschka, F. R. Perpetuation of the Lyme disease spirochete Borrelia lusitaniae by lizards. Appl. Environ. Microbiol. 72, 4627-4632, doi:10.1128/aem.00285-06 (2006).

105 Schmidt, S. et al. Multiple Infections of Rodents with Zoonotic Pathogens in Austria. Vector Borne Zoonotic Dis. 14, 467-475, doi:10.1089/vbz.2013.1504 (2014).

106 Silaghi, C. et al. Babesia spp. and Anaplasma phagocytophilum in questing ticks, ticks parasitizing rodents and the parasitized rodents - Analyzing the host-pathogen-vector interface in a metropolitan area. Parasit. Vectors 5, 191 (2012).

107 Siński, E., Pawełczyk, A., Bajer, A. \& Behnke, J. M. Abundance of wild rodents, ticks and environmental risk of Lyme borreliosis: A longitudinal study in an area of Mazury Lakes district of Poland. Ann. Agric. Environ. Med. 13, 295-300 (2006).

108 Tälleklint, L. \& Jaenson, T. G. T. Infestation of mammals by Ixodes ricinus ticks (Acari: Ixodidae) in south-central Sweden. Exp. Appl. Acarol. 21, 755-771, doi:10.1023/a:1018473122070 (1997).

109 Vourc'h, G., Marmet, J., Chassagne, M., Bord, S. \& Chapuis, J. L. Borrelia burgdorferi sensu lato in Siberian chipmunks (Tamias sibiricus) introduced in suburban forests in France. Vector Borne Zoonotic Dis. 7, 637-641, doi:10.1089/vbz.2007.0111 (2007).

110 Wegner, Z., Stańczak, J., Racewicz, M., Kubica-Biernat, B. \& Kruminis-Łozowska, W. The etiological agent of lyme disease, Borrelia burgdorferi, in ticks (Acari: Ixodidae) from eastern Poland. Zentralbl. Bakteriol. 286, 93-106 (1997).

111 Welc-Faleciak, R., Bajer, A., Behnke, J. M. \& Sinski, E. Effects of host diversity and the community composition of hard ticks (Ixodidae) on Babesia microti infection. Int. J. Med. Microbiol. 298, 235-242, doi:10.1016/j.ijmm.2007.12.002 (2008).

112 Christova, I. \& Gladnishka, T. Prevalence of infection with Francisella tularensis, Borrelia burgdorferi sensu lato and Anaplasma phagocytophilum in rodents from an endemic focus of tularemia in Bulgaria. Ann. Agric. Environ. Med. 12, 149-152 (2005).

113 Dubska, L. et al. Synanthropic Birds Influence the Distribution of Borrelia Species: Analysis of Ixodes ricinus Ticks Feeding on Passerine Birds. Appl. Environ. Microbiol. 77, 1115-1117, doi:10.1128/ aem.02278-10 (2011).

114 James, M. C., Furness, R. W., Bowman, A. S., Forbes, K. J. \& Gilbert, L. The importance of passerine birds as tick hosts and in the transmission of Borrelia burgdorferi, the agent of Lyme disease: a case study from Scotland. Ibis 153, 293-302, doi:10.1111/j.1474-919X.2011.01111.x (2011).

115 Michalik, J., Wodecka, B., Skoracki, M., Sikora, B. \& Stańczak, J. Prevalence of avian-associated Borrelia burgdorferi s.I. genospecies in Ixodes ricinus ticks collected from blackbirds (Turdus merula) and song thrushes (T. philomelos). Int. J. Med. Microbiol. 298, Supplement 1, 129-138 (2008).

116 Taragel'ova, V. et al. Blackbirds and song thrushes constitute a key reservoir of Borrelia garinii, the causative agent of Borreliosis in Central Europe. Appl. Environ. Microbiol. 74, 1289-1293, doi:10.1128/ aem.01060-07 (2008).

117 Kaiser, A., Seitz, A. \& Strub, O. Prevalence of Borrelia burgdorferi sensu lato in the Nightingale (Luscinia megarhynchos) and other passerine birds. Int. J. Med. Microbiol. 291, 75-79 (2002). 


\section{6 | Bibliography}

118 Kipp, S., Goedecke, A., Dorn, W., Wilske, B. \& Fingerle, V. Role of birds in Thuringia, Germany, in the natural cycle of Borrelia burgdorferi sensu lato, the Lyme disease spirochaete. Int. J. Med. Microbiol. 296, 125-128, doi:10.1016/j.jimm.2006.01.041 (2006).

119 Dubska, L. et al. Tick-borne zoonotic pathogens in ticks feeding on the common nightingale including a novel strain of Rickettsia sp. Ticks Tick Borne Dis. 3, 265-268, doi:10.1016/j.ttbdis.2012.06.001 (2012).

120 Mogl, C., de Mendonça, P. G., Harsch, A. \& Hey, J. Potential for Cofeeding Transmission of Tick-Borne Pathogens in Common Voles and Roe Deer - Supportive Molecular Evidence from Field Samples. Acta Zoologica Bulgarica 63, 301-306 (2011).

121 Amore, G. et al. Borrelia lusitaniae in Immature Ixodes ricinus (Acari: Ixodidae) feeding on common wall lizards in Tuscany, central Italy. J. Med. Entomol. 44, 303-307 (2007).

122 Dubska, L., Literak, I., Kocianova, E., Taragelova, V. \& Sychra, O. Differential Role of Passerine Birds in Distribution of Borrelia Spirochetes, Based on Data from Ticks Collected from Birds during the Postbreeding Migration Period in Central Europe. Appl. Environ. Microbiol. 75, 596-602, doi:10.1128/ aem.01674-08 (2009).

123 Fietz, J., Tomiuk, J., Matuschka, F. R. \& Richter, D. Seasonal Prevalence of Lyme Disease Spirochetes in a Heterothermic Mammal, the Edible Dormouse (Glis glis). Appl. Environ. Microbiol. 80, 3615-3621, doi:10.1128/aem.00251-14 (2014).

124 Mannelli, A. et al. Borrelia burgdorferi sensu lato infection in larval Ixodes ricinus (Acari: Ixodidae) feeding on blackbirds in northwestern Italy. J. Med. Entomol. 42, 168-175 (2005).

125 Humair, P. F., Turrian, N., Aeschlimann, A. \& Gern, L. Ixodes ricinus immatures on birds in a focus of Lyme borreliosis. Folia Parasitol. 40, 237-242 (1993).

126 Heylen, D., Tijsse, E., Fonville, M., Matthysen, E. \& Sprong, H. Transmission dynamics of Borrelia burgdorferi s.I. in a bird tick community. Environ. Microbiol. 15, 663-673, doi:10.1111/1462-2920.12059 (2013).

127 Franke, J. et al. Coexistence of pathogens in host-seeking and feeding ticks within a single natural habitat in central Germany. Appl. Environ. Microbiol. 76, 6829-6836, doi:10.1128/aem.01630-10 (2010).

128 Bown, K. J. et al. Sympatric Ixodes trianguliceps and Ixodes ricinus ticks feeding on field voles (Microtus agrestis): Potential for increased risk of Anaplasma phagocytophilum in the United Kingdom? Vector Borne Zoonotic Dis. 6, 404-410, doi:10.1089/vbz.2006.6.404 (2006).

129 Richter, D., Schlee, D. B., Allgower, R. \& Matuschka, F. R. Relationships of a novel Lyme disease spirochete, Borrelia spielmani sp nov., with its hosts in central Europe. Appl. Environ. Microbiol. 70, 6414 6419, doi:10.1128/aem.70.11.6414-6419.2004 (2004).

130 Václav, R., Ficová, M., Prokop, P. \& Betáková, T. Associations Between Coinfection Prevalence of Borrelia lusitaniae, Anaplasma sp., and Rickettsia sp. in Hard Ticks Feeding on Reptile Hosts. Microb. Ecol. 61, 245-253, doi:10.1007/s00248-010-9736-0 (2011).

131 Pacilly, F. C. A. et al. Blood feeding on large grazers affects the transmission of Borrelia burgdorferi sensu lato by Ixodes ricinus. Ticks Tick Borne Dis. 5, 810-817, doi:10.1016/j.ttbdis.2014.06.004 (2014).

132 Heidrich, J. Untersuchungen zur Prävalenz von Borrelia burgdorferi sensu lato beim Rotfuchs (Vulpes vulpes) in Ostbrandenburg PhD thesis, Freien Universität Berlin, (2000).

133 Heidrich, J. et al. Investigation of skin samples from red foxes (Vulpes vulpes) in eastern Brandenburg (Germany) for the detection of Borrelia burgdorferi s. I. Zentralbl. Bakteriol. 289, 666-672 (1999). 
134 Vazquez, L. et al. Tick infestation (Acari: Ixodidae) in roe deer (Capreolus capreolus) from northwestern Spain: population dynamics and risk stratification. Exp. Appl. Acarol. 53, 399-409, doi:10.1007/ s10493-010-9403-7 (2011).

135 Vor, T., Kiffner, C., Hagedorn, P., Niedrig, M. \& Ruhe, F. Tick burden on European roe deer (Capreolus capreolus). Exp. Appl. Acarol. 51, 405-417, doi:10.1007/s10493-010-9337-0 (2010).

136 Tijsse-Klasen, E., Fonville, M., Reimerink, J. H. J., Spitzen-van der Sluijs, A. \& Sprong, H. Role of sand lizards in the ecology of Lyme and other tick-borne diseases in the Netherlands. Parasit. Vectors 3, 42, doi:10.1186/1756-3305-3-42 (2010).

137 De Boer, R., Hovius, K. E., Nohlmans, M. K. \& Gray, J. S. The woodmouse (Apodemus sylvaticus) as a reservoir of tick-transmitted spirochetes (Borrelia burgdorferi) in The Netherlands. Zentralbl. Bakteriol. 279, 404-416 (1993).

138 De Carvalho, I. L. et al. Molecular characterization of a new isolate of Borrelia lusitaniae derived from Apodemus sylvaticus in Portugal. Vector Borne Zoonotic Dis. 10, 531-534, doi:10.1089/vbz.2008.0210 (2010).

139 Gray, J. S., Kahl, O., Janetzki, C. \& Stein, J. Studies on the Ecology of Lyme Disease in a Deer Forest in County Galway, Ireland. J. Med. Entomol. 29, 915-920 (1992).

140 Gray, J. S., Kahl, O., Janetzki, C., Stein, J. \& Guy, E. The spatial distribution of Borrelia burgdorferi-infected Ixodes ricinus in the Connemara region of County Galway, Ireland. Exp. Appl. Acarol. 19, 163-172, doi:10.1007/bf00046288 (1995).

141 Gray, J. S., Robertson, J. N. \& Key, S. Limited role of rodents as reservoirs of Borrelia burgdorferi sensu lato in Ireland. Eur. J. Epidemiol. 16, 101-103, doi:10.1023/a:1007642908546 (2000).

142 Harrison, A., Bown, K. J., Montgomery, W. I. \& Birtles, R. J. Ixodes ricinus is not an epidemiologically relevant vector of Bartonella species in the wood mouse (Apodemus sylvaticus). Vector Borne Zoonotic Dis. 12, 366-371, doi:10.1089/vbz.2011.0807 (2012).

143 Ferreri, L. et al. Pattern of Tick Aggregation on Mice: Larger Than Expected Distribution Tail Enhances the Spread of Tick-Borne Pathogens. PLoS Comp. Biol. 10, e1003931, doi:10.1371/journal.pcbi.1003931 (2014).

144 Michalik, J. et al. Borrelia burgdorferi sensu stricto in yellow-necked mice and feeding Ixodes ricinus ticks in a forest habitat of west central Poland. J. Med. Entomol. 42, 850-856 (2005).

145 Anderson, J. F. \& Magnarelli, L. A. Epizootiology of Lyme Disease-Causing Borreliae. Clinics in Dermatology 11, 339-351 (1993).

146 Rózsa, L., Reiczigel, J. \& Majoros, G. Quantifying parasites in samples of hosts. J. Parasitol. 86, 228-232, doi:10.1645/0022-3395(2000)086[0228:qpisoh]2.0.co;2 (2000).

147 Apollonio, M., Andersen, R. \& Putman, R. European ungulates and their management in the 21st century. 618 (Cambridge University Press, 2010).

148 Gregory, R. D. et al. Population trends of widespread woodland birds in Europe. Ibis 149, 78-97, doi:10.1111/j.1474-919X.2007.00698.x (2007).

149 van Strien, A. J., Bekker, D. L., La Haye, M. J. J. \& van der Meij, T. Trends in small mammals derived from owl pellet data using occupancy modelling. Mammalian Biology - Zeitschrift für Säugetierkunde 80, 340-346, doi:http://dx.doi.org/10.1016/j.mambio.2015.04.002 (2015).

150 Hulinska, D. et al. Molecular and microscopical evidence of Ehrlichia spp. and Borrelia burgdorferi sensu lato in patients, animals and ticks in the Czech Republic. New Microbiol. 25, 437-448 (2002).

151 Pato, F. J. et al. Seroprevalence of borrelia burgdorferi sensu lato in roe deer (Capreolus Capreolus) from Northwestern Spain. J. Zoo Wildl. Med. 44, 660-665, doi:10.1638/2012-0240r2.1 (2013). 
152 Pichon, B., Gilot, B. \& Perez-Eid, C. Detection of spirochaetes of Borrelia burgdorferi complexe in the skin of cervids by PCR and culture. Eur. J. Epidemiol. 16, 869-873 (2000).

153 Kjelland, V., Ytrehus, B., Stuen, S., Skarpaas, T. \& Slettan, A. Prevalence of Borrelia burgdorferi in Ixodes ricinus ticks collected from moose (Alces alces) and roe deer (Capreolus capreolus) in southern Norway. Ticks Tick Borne Dis. 2, 99-103, doi:10.1016/j.ttbdis.2010.12.002 (2011).

154 Matuschka, F. R. et al. Diversionary role of hoofed game in the transmission of Lyme disease spirochetes. Am. J. Trop. Med. Hyg. 48, 693-699 (1993).

155 Martin, L. B., Weil, Z. M. \& Nelson, R. J. Immune defense and reproductive pace of life in Peromyscus mice. Ecology 88, 2516-2528, doi:10.1890/07-0060.1 (2007).

156 Gassner, F. et al. Geographic and Temporal Variations in Population Dynamics of Ixodes ricinus and Associated Borrelia Infections in The Netherlands. Vector Borne Zoonotic Dis. 11, 523-532, doi:10.1089/ vbz.2010.0026 (2011).

157 Ostfeld, R. S. \& Keesing, F. Biodiversity and disease risk: The case of lyme disease. Conserv. Biol. 14, 722-728, doi:10.1046/j.1523-1739.2000.99014.x (2000).

158 Randolph, S. E. \& Dobson, A. D. M. Pangloss revisited: a critique of the dilution effect and the biodiversity-buffers-disease paradigm. Parasitology 139, 847-863, doi:doi:10.1017/S0031182012000200 (2012).

159 Wood, C. L. \& Lafferty, K. D. Biodiversity and disease: A synthesis of ecological perspectives on Lyme disease transmission. Trends Ecol. Evol. 28, 239-247, doi:10.1016/j.tree.2012.10.011 (2013).

160 Ostfeld, R. S. \& Holt, R. D. Are predators good for your health? Evaluating evidence for top-down regulation of zoonotic disease reservoirs. Front. Ecol. Environ. 2, 13-20, doi:10.1890/1540-9295(2004)002[ 0013:apgfyh]2.0.co;2 (2004).

161 Humair, P. F. \& Gern, L. Relationship between Borrelia burgdorferi sensu lato species, red squirrels (Sciurus vulgaris) and Ixodes ricinus in enzootic areas in Switzerland. Acta Trop. 69, 213-227, doi:10.1016/ s0001-706x(97)00126-5 (1998).

162 Pisanu, B. et al. High prevalence of Borrelia burgdorferi s.I. in the European red squirrel Sciurus vulgaris in France. Ticks Tick Borne Dis. 5, 1-6, doi:http://dx.doi.org/10.1016/j.ttbdis.2013.07.007 (2014).

163 Wilson, G. J. \& Delahay, R. J. A review of methods to estimate the abundance of terrestrial carnivores using field signs and observation. Wildl. Res. 28, 151-164, doi:http://dx.doi.org/10.1071/WR00033 (2001).

164 Rovero, F., Zimmermann, F., Berzi, D. \& Meek, P. “Which camera trap type and how many do I need?” A review of camera features and study designs for a range of wildlife research applications. Hystrix 24, doi:10.4404/hystrix-24.2-6316 (2013).

165 Karanth, K. U. et al. Science deficiency in conservation practice: The monitoring of tiger populations in India. Anim. Conserv. 6, 141-146 (2003).

166 Carbone, C. et al. The use of photographic rates to estimate densities of tigers and other cryptic mammals. Anim. Conserv. 4, 75-79 (2001).

167 Sollmann, R., Mohamed, A., Samejima, H. \& Wilting, A. Risky business or simple solution - Relative abundance indices from camera-trapping. Biol. Conserv. 159, 405-412, doi:10.1016/j.biocon.2012.12.025 (2013).

168 Kelly, M. J. \& Holub, E. L. Camera Trapping of Carnivores: Trap Success Among Camera Types and Across Species, and Habitat Selection by Species, on Salt Pond Mountain, Giles County, Virginia. Northeast. Nat. 15, 249-262, doi:10.1656/1092-6194(2008)15[249:ctocts]2.0.co;2 (2008).

169 McNab, B. K. On Estimating Thermal Conductance in Endotherms. Physiol. Zool. 53, 145-156 (1980). 
170 Rowcliffe, J. M., Field, J., Turvey, S. T. \& Carbone, C. Estimating animal density using camera traps without the need for individual recognition. J. Appl. Ecol. 45, 1228-1236, doi:10.1111/j.13652664.2008.01473.x (2008).

171 Caravaggi, A. et al. An invasive-native mammalian species replacement process captured by camera trap survey random encounter models. Remote Sensing in Ecology and Conservation 2, 45-58, doi:10.1002/ rse2.11 (2016).

172 Buckland, S. T., Rexstad, E. A., Marques, T. A. \& Oedekoven, C. S. Distance Sampling: Methods and Applications. (Springer International Publishing, 2015).

173 Buckland, S. T. et al. Introduction to Distance Sampling. (Oxford University Press, 2001).

174 Thomas, L. et al. Distance software: design and analysis of distance sampling surveys for estimating population size. J. Appl. Ecol. 47, 5-14, doi:10.1111/j.1365-2664.2009.01737.x (2010).

175 mrds: Mark-Recapture Distance Sampling v. R package version 2.1.14 (http://CRAN.R-project.org/package $=$ mrds, 2015)

176 Marques, F. F. C. \& Buckland, S. T. Incorporating Covariates into Standard Line Transect Analyses. Biometrics 59, 924-935, doi:10.1111/j.0006-341X.2003.00107.x (2003).

177 Meek, P. D. et al. Recommended guiding principles for reporting on camera trapping research. Biodivers. Conserv. 23, 2321-2343, doi:10.1007/s10531-014-0712-8 (2014).

178 Kays, R. et al. in 2009 IEEE 34th Conference on Local Computer Networks, LCN 2009. 811-818.

179 Jones, K. E. et al. PanTHERIA: a species-level database of life history, ecology, and geography of extant and recently extinct mammals. Ecology 90, 2648-2648, doi:10.1890/08-1494.1 (2009).

180 Tobler, M. W., Carrillo-Percastegui, S. E., Leite Pitman, R., Mares, R. \& Powell, G. An evaluation of camera traps for inventorying large- and medium-sized terrestrial rainforest mammals. Anim. Conserv. 11, 169178, doi:10.1111/j.1469-1795.2008.00169.x (2008).

181 Anile, S. \& Devillard, S. Study design and body mass influence RAls from camera trap studies: evidence from the Felidae. Anim. Conserv. 19, 35-45, doi:10.1111/acv.12214 (2016).

182 Rovero, F. \& Marshall, A. R. Camera trapping photographic rate as an index of density in forest ungulates. J. Appl. Ecol. 46, 1011-1017, doi:10.1111/j.1365-2664.2009.01705.x (2009).

183 Manzo, E., Bartolommei, P., Rowcliffe, J. M. \& Cozzolino, R. Estimation of population density of European pine marten in central Italy using camera trapping. Acta Theriologica 57, 165-172, doi:10.1007/ s13364-011-0055-8 (2012).

184 Rowcliffe, J. M., Kays, R., Carbone, C. \& Jansen, P. A. Clarifying assumptions behind the estimation of animal density from camera trap rates. The Journal of Wildlife Management 77, 876-876, doi:10.1002/ jwmg.533 (2013).

185 Cusack, J. J. et al. Applying a random encounter model to estimate lion density from camera traps in Serengeti National Park, Tanzania. The Journal of Wildlife Management 79, 1014-1021, doi:10.1002/ jwmg.902 (2015).

186 Rowcliffe, J. M., Jansen, P. A., Kays, R., Kranstauber, B. \& Carbone, C. Wildlife speed cameras: measuring animal travel speed and day range using camera traps. Remote Sensing in Ecology and Conservation, n/a-n/a, doi:10.1002/rse2.17 (2016).

187 Stephens, P. A., Zaumyslova, O. Y., Miquelle, D. G., Myslenkov, A. I. \& Hayward, G. D. Estimating population density from indirect sign: Track counts and the Formozov-Malyshev-Pereleshin formula. Anim. Conserv. 9, 339-348, doi:10.1111/j.1469-1795.2006.00044.x (2006).

188 Kilpatrick, A. M. \& Randolph, S. E. Drivers, dynamics, and control of emerging vector-borne zoonotic diseases. The Lancet 380, 1946-1955, doi:http://dx.doi.org/10.1016/S0140-6736(12)61151-9 (2012). 
189 Sonenshine, D. E. Biology of ticks. (Oxford University Press, 1991).

190 Hofmeester, T. R. et al. Few vertebrate species dominate the Borrelia burgdorferi s.l. life cycle. Environmental Research Letters 11, 043001, doi:10.1088/1748-9326/11/4/043001 (2016).

191 Rand, P. W. et al. Deer density and the abundance of Ixodes scapularis (Acari : Ixodidae). J. Med. Entomol. 40, 179-184, doi:10.1603/0022-2585-40.2.179 (2003).

192 Rand, P. W., Lubelczyk, C., Holman, M. S., Lacombe, E. H. \& Smith, R. P. Abundance of Ixodes scapularis (Acari : Ixodidae) after the complete removal of deer from an isolated offshore island, endemic for Lyme disease. J. Med. Entomol. 41, 779-784, doi:10.1603/0022-2585-41.4.779 (2004).

193 Kugeler, K. J., Jordan, R. A., Schulze, T. L., Griffith, K. S. \& Mead, P. S. Will Culling White-Tailed Deer Prevent Lyme Disease? Zoonoses and Public Health, doi:10.1111/zph.12245 (2015).

194 Perkins, S. E., Cattadori, I. M., Tagliapietra, V., Rizzoli, A. P. \& Hudson, P. J. Localized deer absence leads to tick amplification. Ecology 87, 1981-1986, doi:10.1890/0012-9658(2006)87[1981:Idaltt]2.0.co;2 (2006).

195 Dobson, A. D. M. \& Randolph, S. E. Modelling the effects of recent changes in climate, host density and acaricide treatments on population dynamics of Ixodes ricinus in the UK. J. Appl. Ecol. 48, 1029-1037, doi:10.1111/j.1365-2664.2011.02004.x (2011).

196 Li, S., Vanwambeke, S. O., Licoppe, A. M. \& Speybroeck, N. Impacts of deer management practices on the spatial dynamics of the tick Ixodes ricinus: A scenario analysis. Ecol. Model. 276, 1-13, doi:10.1016/j. ecolmodel.2013.12.023 (2014).

197 Broekhuizen, S., Spoelstra, K., Thissen, J. B. M., Canters, K. J. \& Buys, J. C. Atlas van de Nederlandse Zoogdieren. (KNNV Uitgeverij, 2016).

198 Hofmeester, T. R., Rowcliffe, J. M. \& Jansen, P. A. A simple method for estimating the effective detection distance of camera traps. Remote Sensing in Ecology and Conservation (Accepted).

199 Tack, W. et al. The effects of sampling method and vegetation type on the estimated abundance of Ixodes ricinus ticks in forests. Exp. Appl. Acarol. 54, 285-292, doi:10.1007/s10493-011-9444-6 (2011).

200 Fournier, D. A. et al. AD Model Builder: Using automatic differentiation for statistical inference of highly parameterized complex nonlinear models. Optimization Methods and Software 27, 233-249, doi:10.10 80/10556788.2011.597854 (2012).

201 Generalized Linear Mixed Models using AD Model Builder (http://glmmadmb.r-forge.r-project.org/, 2015).

202 Wilson, M. L., Telford, S. R., Piesman, J. \& Spielman, A. Reduced Abundance of Immature Ixodes dammini (Acari: Ixodidae) Following Elimination of Deer. J. Med. Entomol. 25, 224-228, doi:10.1093/jmedent/25.4.224 (1988).

203 Dobson, A. History and complexity in tick-host dynamics: discrepancies between 'real' and 'visible' tick populations. Parasit. Vectors 7, 231 (2014).

204 Ostfeld, R. S., Canham, C. D., Oggenfuss, K., Winchcombe, R. J. \& Keesing, F. Climate, deer, rodents, and acorns as determinants of variation in Lyme-disease risk. PLoS Biol. 4, 1058-1068, doi:10.1371/ journal.pbio.0040145 (2006).

205 Tagliapietra, V. et al. Saturation deficit and deer density affect questing activity and local abundance of Ixodes ricinus (Acari, Ixodidae) in Italy. Vet. Parasitol. 183, 114-124, doi:10.1016/j.vetpar.2011.07.022 (2011).

206 Gilbert, L. Altitudinal patterns of tick and host abundance: a potential role for climate change in regulating tick-borne diseases? Oecologia 162, 217-225, doi:10.1007/s00442-009-1430-x (2010). 
207 Ruiz-Fons, F. \& Gilbert, L. The role of deer as vehicles to move ticks, Ixodes ricinus, between contrasting habitats. Int. J. Parasitol. 40, 1013-1020, doi:10.1016/j.ijpara.2010.02.006 (2010).

208 James, M. C. et al. Environmental determinants of Ixodes ricinus ticks and the incidence of Borrelia burgdorferi sensu lato, the agent of Lyme borreliosis, in Scotland. Parasitology 140, 237-246, doi:doi:10.1017/S003118201200145X (2013).

209 Gilbert, L., Jones, L. D., Hudson, P. J., Gould, E. A. \& Reid, H. W. Role of small mammals in the persistence of Louping-ill virus: field survey and tick co-feeding studies. Med. Vet. Entomol. 14, 277-282, doi:10.1046/j.1365-2915.2000.00236.x (2000).

210 Crofton, H. D. A quantitative approach to parasitism. Parasitology 62, 179-193, doi:doi:10.1017/ S0031182000071420 (1971).

211 Anderson, R. M. \& May, R. M. Regulation and Stability of Host-Parasite Population Interactions: I. Regulatory Processes. J. Anim. Ecol. 47, 219-247, doi:10.2307/3933 (1978).

212 Haskell, J. P., Ritchie, M. E. \& Olff, H. Fractal geometry predicts varying body size scaling relationships for mammal and bird home ranges. Nature 418, 527-530, doi:10.1038/nature00840 (2002).

213 Tew, T. E. \& Macdonald, D. W. Dynamics of space use and male vigour amongst wood mice, Apodemus sylvaticus, in the cereal ecosystem. Behav. Ecol. Sociobiol. 34, 337-345, doi:10.1007/bf00197004 (1994).

214 Borowski, Z. \& Owadowska, E. Field vole (Microtus agrestis) seasonal spacing behavior: The effect of predation risk by mustelids. Naturwissenschaften 97, 487-493, doi:10.1007/s00114-010-0663-1 (2010).

215 Harrison, A., Scantlebury, M. \& Montgomery, W. I. Body mass and sex-biased parasitism in wood mice Apodemus sylvaticus. Oikos 119, 1099-1104, doi:10.1111/j.1600-0706.2009.18072.x (2010).

216 Lindenfors, P. et al. Parasite species richness in carnivores: Effects of host body mass, latitude, geographical range and population density. Global Ecol. Biogeogr. 16, 496-509, doi:10.1111/j.14668238.2006.00301.x (2007).

217 Morand, S., Goüy de Bellocq, J., Stanko, M. \& Miklisová, D. Is sex-biased ectoparasitism related to sexual size dimorphism in small mammals of Central Europe? Parasitology 129, 505-510, doi:doi:10.1017/ S0031182004005840 (2004).

218 Johnson, P. T. J., de Roode, J. C. \& Fenton, A. Why infectious disease research needs community ecology. Science 349, doi:10.1126/science.1259504 (2015).

219 Lange, R., Twisk, P., van Winden, A. \& van Diepenbeek, A. Zoogdieren van West-Europa. (KNNV, 2003).

220 Hillyard, P. D. Ticks of North-West Europe. (Backhuys Publishers, 1996).

221 Otis, D. L., Burnham, K. P., White, G. C. \& Anderson, D. R. Statistical Inference from Capture Data on Closed Animal Populations. Wildlife Monographs, 3-135, doi:10.2307/3830650 (1978).

222 White, G. C. \& Burnham, K. P. Program MARK: Survival estimation from populations of marked animals. Bird Study 46, S120-S139 (1999).

223 Gelman, A. Scaling regression inputs by dividing by two standard deviations. Stat. Med. 27, 2865-2873, doi:10.1002/sim.3107 (2008).

224 Zuur, A. F., leno, E. N. \& Elphick, C. S. A protocol for data exploration to avoid common statistical problems. Methods in Ecology and Evolution 1, 3-14, doi:10.1111/j.2041-210X.2009.00001.x (2010).

225 Zuur, A. F., leno, E. N., Walker, N. J., Saveliev, A. A. \& Smith, G. M. in Mixed effects models and extensions in ecology with $R \quad$ 261-293 (Springer, 2009).

226 Symonds, M. R. E. \& Moussalli, A. A brief guide to model selection, multimodel inference and model averaging in behavioural ecology using Akaike's information criterion. Behav. Ecol. Sociobiol. 65, 13-21, doi:10.1007/s00265-010-1037-6 (2011). 
Rosa, R., Pugliese, A., Ghosh, M., Perkins, S. E. \& Rizzoli, A. Temporal variation of Ixodes ricinus intensity on the rodent host Apodemus flavicollis in relation to local climate and host dynamics. Vector Borne Zoonotic Dis. 7, 285-295, doi:10.1089/vbz.2006.0607 (2007).

228 Díaz, M., Torre, I., Peris, A. \& Tena, L. Foraging behavior of wood mice as related to presence and activity of genets. J. Mammal. 86, 1178-1185, doi:10.1644/04-mamm-a-127r1.1 (2005).

229 Haapakoski, M., Sundell, J. \& Ylönen, H. Conservation implications of change in antipredator behavior in fragmented habitat: Boreal rodent, the bank vole, as an experimental model. Biol. Conserv. 184, 11-17, doi:http://dx.doi.org/10.1016/j.biocon.2014.12.023 (2015).

230 LoGiudice, K. et al. Impact of Host Community Composition on Lyme Disease Risk. Ecology 89, 28412849, doi:10.1890/07-1047.1 (2008).

231 O'Brien, T. G. \& Kinnaird, M. F. A picture is worth a thousand words: the application of camera trapping to the study of birds. Bird Conservation International 18, S144-S162, doi:doi:10.1017/ S0959270908000348 (2008).

232 Schouls, L. M., Van De Pol, I., Rijpkema, S. G. T. \& Schot, C. S. Detection and identification of Ehrlichia, Borrelia burgdorferi sensu lato, and Bartonella species in Dutch Ixodes ricinus ticks. J. Clin. Microbiol. 37, 2215-2222 (1999).

233 Jahfari, S. et al. Prevalence of Neoehrlichia mikurensis in ticks and rodents from North-west Europe. Parasites and Vectors 5, doi:10.1186/1756-3305-5-74 (2012).

234 Coipan, E. C. et al. Geodemographic analysis of Borrelia burgdorferi sensu lato using the 5S-23S rDNA spacer region. Infect., Genet. Evol. 17, 216-222 (2013).

235 Sanger, F., Nicklen, S. \& Coulson, A. R. DNA sequencing with chain-terminating inhibitors. Proceedings of the National Academy of Sciences of the United States of America 74, 5463-5467 (1977).

236 nlme: Linear and Nonlinear Mixed Effects Models v. R package version 3.1-122 (http://CRAN.R-project. org/package=nlme, 2015).

237 Shipley, B. Confirmatory path analysis in a generalized multilevel context. Ecology 90, 363-368, doi:10.1890/08-1034.1 (2009).

238 Tälleklint, L. \& Jaenson, T. G. T. Maintenance by hares of European Borrelia Burgdorferi in ecosystems without rodents. J. Med. Entomol. 30, 273-276 (1993).

239 Tälleklint, L. \& Jaenson, T. G. T. Relationship between Ixodes ricinus density and prevalence of infection with Borrelia-like spirochetes and density of infected ticks. J. Med. Entomol. 33, 805-811 (1996).

240 Jaenson, T. G. T. \& Tälleklint, L. Incompetence of Roe Deer as Reservoirs of the Lyme Borreliosis Spirochete. J. Med. Entomol. 29, 813-817 (1992).

241 Levi, T., Keesing, F., Holt, R. D., Barfield, M. \& Ostfeld, R. S. Quantifying dilution and amplification in a community of hosts for tick-borne pathogens. Ecol. Appl. 26, 484-498, doi:10.1890/15-0122 (2016).

242 Davis, S., Abbasi, B., Shah, S., Telfer, S. \& Begon, M. Spatial analyses of wildlife contact networks. Journal of The Royal Society Interface 12, doi:10.1098/rsif.2014.1004 (2015).

243 Korpimäki, E., Norrdahl, K., Klemola, T., Pettersen, T. \& Stenseth, N. C. Dynamic effects of predators on cyclic voles: field experimentation and model extrapolation. Proceedings of the Royal Society of London B: Biological Sciences 269, 991-997, doi:10.1098/rspb.2002.1972 (2002).

244 O’Mahony, D., Lambin, X., MacKinnon, J. L. \& Coles, C. F. Fox predation on cyclic field vole populations in Britain. Ecography 22, 575-581, doi:10.1111/j.1600-0587.1999.tb01287.x (1999).

245 Krebs, C. J. Population Fluctuations in Rodents. (The University of Chicago Press, 2013).

246 Christian, A. in Milbenkundlichen Kolloquium (Karl-Franzens-Universität Graz im Institut für Zoologie, 2001). 
247

248

249

250

251

252

253

254

255

256

257

258

259

260

261 Jaenson, T. G. T. et al. Risk indicators for the tick Ixodes ricinus and Borrelia burgdorferi sensu lato in Sweden. Med. Vet. Entomol. 23, 226-237 (2009).

262 Mysterud, A., Easterday, W. R., Qviller, L., Viljugrein, H. \& Ytrehus, B. Spatial and seasonal variation in the prevalence of Anaplasma phagocytophilum and Borrelia burgdorferi sensu lato in questing Ixodes ricinus ticks in Norway. Parasites and Vectors 6, doi:10.1186/1756-3305-6-187 (2013).

263 Mysterud, A. et al. Contrasting emergence of Lyme disease across ecosystems. Nature Communications 7 (2016).

264 Heylen, D., Adriaensen, F., Van Dongen, S., Sprong, H. \& Matthysen, E. Ecological factors that determine Ixodes ricinus tick burdens in the great tit (Parus major), an avian reservoir of Borrelia burgdorferi s.l. Int. J. Parasitol. 43, 603-611, doi:10.1016/j.ijpara.2013.02.007 (2013). 
265 Nupp, T. E. \& Swihart, R. K. Landscape-level correlates of small-mammal assemblages in forest fragments of farmland. J. Mammal. 81, 512-526, doi:10.1644/1545-1542(2000)081<0512:Ilcosm>2.0. co;2 (2000).

266 Tews, J. et al. Animal species diversity driven by habitat heterogeneity/diversity: the importance of keystone structures. J. Biogeogr. 31, 79-92, doi:10.1046/j.0305-0270.2003.00994.x (2004).

267 Villette, P., Krebs, C. J., Jung, T. S. \& Boonstra, R. Can camera trapping provide accurate estimates of small mammal (Myodes rutilus and Peromyscus maniculatus) density in the boreal forest? J. Mammal., doi:10.1093/jmammal/gyv150 (2015).

268 Grenfell, B. T. \& A. P. Dobson. Ecology of Infectious Diseases in Natural Populations. (Cambridge University Press, 1995).

269 Dobson, A. Population dynamics of pathogens with multiple host species. Am. Nat. 164, S64-S78, doi:10.1086/424681 (2004).

270 Salkeld, D. J., Padgett, K. A. \& Jones, J. H. A meta-analysis suggesting that the relationship between biodiversity and risk of zoonotic pathogen transmission is idiosyncratic. Ecol. Lett., doi:10.1111/ele.12101 (2013).

271 Wood, C. L. et al. Does biodiversity protect humans against infectious disease? Ecology 95, 817-832, doi:10.1890/13-1041.1 c2 - 24933803 (2014).

272 Swart, A. et al. Predicting tick presence by environmental risk mapping. Frontiers in public health 2, 238, doi:10.3389/fpubh.2014.00238 (2014).

273 Ostfeld, R. S. \& LoGiudice, K. Community disassembly, biodiversity loss, and the erosion of an ecosystem service. Ecology 84, 1421-1427, doi:10.1890/02-3125 (2003).

274 Kurtenbach, K. et al. The key roles of selection and migration in the ecology of Lyme borreliosis. Int. J. Med. Microbiol. 291, 152-154 (2002).

275 Craft, M. E. Infectious disease transmission and contact networks in wildlife and livestock. Philosophical Transactions of the Royal Society of London B: Biological Sciences 370, doi:10.1098/rstb.2014.0107 (2015).

276 Norman, R. A., Worton, A. J. \& Gilbert, L. Past and future perspectives on mathematical models of tickborne pathogens. Parasitology 143, 850-859, doi:doi:10.1017/S0031182015001523 (2016).

277 Cobbold, C. A., Teng, J. \& Muldowney, J. S. The influence of host competition and predation on tick densities and management implications. Theoretical Ecology 8, 349-368, doi:10.1007/s12080-015-0255-y (2015).

278 Balashov, Y. S. Bloodsucking Ticks (Ixodoidea) - Vectors of Diseases of Man and Animals. (Nauka Publishers, 1968).

279 Jamon, M. The dynamics of Wood mouse (Apodemus sylvaticus) populations in the Camargue. J. Zool. 208, 569-582, doi:10.1111/j.1469-7998.1986.tb01524.x (1986).

280 Dobson, A. D. M., Finnie, T. J. R. \& Randolph, S. E. A modified matrix model to describe the seasonal population ecology of the European tick Ixodes ricinus. J. Appl. Ecol. 48, 1017-1028, doi:10.1111/j.13652664.2011.02003.x (2011).

281 Brown, J. S., Laundré, J. W. \& Gurung, M. The Ecology of Fear: Optimal Foraging, Game Theory, and Trophic Interactions. J. Mammal. 80, 385-399, doi:10.2307/1383287 (1999).

282 Prins, H. H. T. \& Langevelde, F. v. in Resource Ecology: Spatial and Temporal Dynamics of Foraging Wageningen UR Frontis Series (eds H.H.T. Prins \& F. van Langevelde) 129-155 (Springer, 2008).

283 Voordouw, M. J., Lachish, S. \& Dolan, M. C. The Lyme Disease Pathogen Has No Effect on the Survival of Its Rodent Reservoir Host. PLoS One 10, e0118265 (2015). 
284 Allan, B. F., Varns, T. S. \& Chase, J. M. Fear of Parasites: Lone star ticks increase giving-up densities in White-tailed deer. Israel Journal of Ecology \& Evolution 56, 313-324, doi:10.1560/ijee.56.3-4.313 (2010).

285 Bolzoni, L., Rosà, R., Cagnacci, F. \& Rizzoli, A. Effect of deer density on tick infestation of rodents and the hazard of tick-borne encephalitis. II: Population and infection models. Int. J. Parasitol. 42, 373-381 (2012).

286 Rosa, R. \& Pugliese, A. Effects of tick population dynamics and host densities on the persistence of tickborne infections. Math. Biosci. 208, 216-240, doi:10.1016/j.mbs.2006.10.002 (2007).

287 Randolph, S. E. Ticks are not insects: Consequences of contrasting vector biology for transmission potential. Parasitol. Today 14, 186-192, doi:10.1016/s0169-4758(98)01224-1 (1998).

288 Hofmeister, E. K., Ellis, B. A., Glass, G. E. \& Childs, J. E. Longitudinal study of infection with Borrelia burgdorferi in a population of Peromyscus leucopus at a Lyme disease-enzootic site in Maryland. The American Journal of Tropical Medicine and Hygiene 60, 598-609 (1999).

289 Hart, B. L. Behavioral adaptations to pathogens and parasites: Five strategies. Neuroscience and Biobehavioral Reviews 14, 273-294, doi:10.1016/s0149-7634(05)80038-7 (1990).

290 Randolph, S. E. Transmission of tick-borne pathogens between co-feeding ticks: Milan Labuda's enduring paradigm. Ticks Tick Borne Dis. 2, 179-182, doi:10.1016/j.ttbdis.2011.07.004 (2011).

291 Randolph, S. E., Miklisova, D., Lysy, J., Rogers, D. J. \& Labuda, M. Incidence from coincidence: patterns of tick infestations on rodents facilitate transmission of tick-borne encephalitis virus. Parasitology 118, 177-186, doi:10.1017/s0031182098003643 (1999).

292 Telfer, S. et al. Species interactions in a parasite community drive infection risk in a wildlife population. Science 330, 243-246, doi:10.1126/science.1190333 (2010).

293 Khalil, H., Ecke, F., Evander, M., Magnusson, M. \& Hörnfeldt, B. Declining ecosystem health and the dilution effect. 6, 31314 (2016).

294 Kershenbaum, A., Stone, L., Kotler, B. P. \& Blaustein, L. Predation Risk can Drive Cycles in Zoonotic Disease Prevalence. Israel Journal of Ecology \& Evolution 56, 281-295, doi:10.1560/ijee.56.3-4.281 (2010).

295 Nathan, R. et al. A movement ecology paradigm for unifying organismal movement research. Proc. Natl. Acad. Sci. USA 105, 19052-19059, doi:10.1073/pnas.0800375105 (2008).

296 Brown, J. S. Patch use under predation risk: I. Models and predictions. Ann. Zool. Fenn. 29, 301-309 (1992).

297 Brown, J. S., Kotler, B. P., Smith, R. J. \& Wirtz li, W. O. The effects of owl predation on the foraging behavior of heteromyid rodents. Oecologia 76, 408-415, doi:10.1007/bf00377036 (1988).

298 Ripple, W. J., Larsen, E. J., Renkin, R. A. \& Smith, D. W. Trophic cascades among wolves, elk and aspen on Yellowstone National Park's northern range. Biol. Conserv. 102, 227-234, doi:http://dx.doi. org/10.1016/S0006-3207(01)00107-0 (2001).

299 Post, P. \& Götmark, F. Foraging behavior and predation risk in male and female Eurasian blackbirds (Turdus merula) during the breeding season. The Auk 123, 162-170, doi:doi:10.1642/0004-8038(2006)123[0162:FBAPRI]2.0.C0;2 (2006).

300 Abrams, P. A. Measuring the population-level consequences of predator-induced prey movement. Evol. Ecol. Res. 10, 333-350 (2008).

301 Eccard, J. A. \& Ylönen, H. Costs of coexistence along a gradient of competitor densities: an experiment with arvicoline rodents. J. Anim. Ecol. 76, 65-71, doi:10.1111/j.1365-2656.2006.01175.x (2007). 
302 Liesenjohann, M. et al. From interference to predation: type and effects of direct interspecific interactions of small mammals. Behav. Ecol. Sociobiol. 65, 2079-2089 (2011).

303 Schradin, C. et al. Female home range size is regulated by resource distribution and intraspecific competition: a long-term field study. Anim. Behav. 79, 195-203, doi:http://dx.doi.org/10.1016/j.anbehav.2009.10.027 (2010).

304 Roy, M. \& Holt, R. D. Effects of predation on host-pathogen dynamics in SIR models. Theor. Popul. Biol. 73, 319-331, doi:http://dx.doi.org/10.1016/j.tpb.2007.12.008 (2008).

305 Packer, C., Holt, R. D., Hudson, P. J., Lafferty, K. D. \& Dobson, A. P. Keeping the herds healthy and alert: implications of predator control for infectious disease. Ecol. Lett. 6, 797-802, doi:10.1046/j.14610248.2003.00500.x (2003).

306 Bouskila, A. A habitat selection game of interactions between rodents and their predators. Ann. Zool. Fenn. 38, 55-70 (2001).

307 Kallio, E. R. et al. Prolonged survival of Puumala hantavirus outside the host: evidence for indirect transmission via the environment. J. Gen. Virol. 87, 2127-2134, doi:doi:10.1099/vir.0.81643-0 (2006).

308 Ostfeld, R. S., Glass, G. E. \& Keesing, F. Spatial epidemiology: an emerging (or re-emerging) discipline. Trends Ecol. Evol. 20, 328-336, doi:10.1016/j.tree.2005.03.009 (2005).

309 Bradley, C. A. \& Altizer, S. Urbanization and the ecology of wildlife diseases. Trends Ecol. Evol. 22, 95102, doi:http://dx.doi.org/10.1016/j.tree.2006.11.001 (2007).

310 Hewison, A. J. et al. The effects of woodland fragmentation and human activity on roe deer distribution in agricultural landscapes. Canadian Journal of Zoology 79, 679-689, doi:10.1139/z01-032 (2001).

311 Michel, N., Burel, F. \& Butet, A. How does landscape use influence small mammal diversity, abundance and biomass in hedgerow networks of farming landscapes? Acta Oecol. 30, 11-20, doi:http://dx.doi. org/10.1016/j.actao.2005.12.006 (2006).

312 Proulx, G. et al. in Martens and Fishers (Martes) in Human-Altered Environments: An International Perspective (eds Daniel J. Harrison, Angela K. Fuller, \& Gilbert Proulx) 21-76 (Springer US, 2005).

313 Chapron, G. et al. Recovery of large carnivores in Europe's modern human-dominated landscapes. Science 346, 1517-1519, doi:10.1126/science.1257553 (2014).

314 Ritchie, E. G. \& Johnson, C. N. Predator interactions, mesopredator release and biodiversity conservation. Ecol. Lett. 12, 982-998, doi:10.1111/j.1461-0248.2009.01347.x (2009).

315 Melis, C. et al. Predation has a greater impact in less productive environments: variation in roe deer, Capreolus capreolus, population density across Europe. Global Ecol. Biogeogr. 18, 724-734, doi:10.1111/ j.1466-8238.2009.00480.x (2009).

316 Tufto, J., Andersen, R. \& Linnell, J. Habitat Use and Ecological Correlates of Home Range Size in a Small Cervid: The Roe Deer. J. Anim. Ecol. 65, 715-724, doi:10.2307/5670 (1996).

317 Møller, A. P. Flight distance of urban birds, predation, and selection for urban life. Behav. Ecol. Sociobiol. 63, 63-75, doi:10.1007/s00265-008-0636-y (2008).

318 Meyer, A. in Rats, Mice and People: Rodent Biology and Management Australian Centre for International Agricultural Research monograph (eds G.R. Singleton, L.A. Hinds, C.J. Krebs, \& D.M. Spratt) (ACIAR, 2003).

319 Wang, M. \& Schreiber, A. The impact of habitat fragmentation and social structure on the population genetics of roe deer (Capreolus capreolus L.) in Central Europe. Heredity 86, 703-715, doi:10.1046/j.1365-2540.2001.t01-1-00889.x (2001). 
320 Gregoire, A., Faivre, B., Heeb, P. \& Cezilly, F. A comparison of infestation patterns by Ixodes ticks in urban and rural populations of the Common Blackbird Turdus merula. Ibis 144, 640-645, doi:10.1046/j.1474919X.2002.00102.x (2002).

321 Matuschka, F. R. et al. Risk of urban lyme disease enhanced by the presence of rats. J. Infect. Dis. 174, 1108-1111 (1996).

322 Morse, S. S. et al. Prediction and prevention of the next pandemic zoonosis. The Lancet 380, 19561965, doi:10.1016/s0140-6736(12)61684-5 (2012).

323 Hof, A. R., Snellenberg, J. \& Bright, P. W. Food or fear? Predation risk mediates edge refuging in an insectivorous mammal. Anim. Behav. 83, 1099-1106, doi:http://dx.doi.org/10.1016/j.anbehav.2012.01.042 (2012).

324 Poel, J. L. v. d., Dekker, J. \& Langevelde, F. v. Dutch hedgehogs Erinaceus europaeus are nowadays mainly found in urban areas, possibly due to the negative Effects of badgers Meles meles. Wildl. Biol. 21, 51-55, doi:10.2981/wlb.00072 (2015).

325 Coipan, E. C. et al. Imbalanced presence of Borrelia burgdorferi s.I. multilocus sequence types in clinical manifestations of Lyme borreliosis. Infect., Genet. Evol. 42, 66-76, doi:http://dx.doi.org/10.1016/j. meegid.2016.04.019 (2016).

326 Skuballa, J. et al. Occurrence of different Borrelia burgdorferi sensu lato genospecies including B. afzelii, B. bavariensis, and B. spielmanii in hedgehogs (Erinaceus spp.) in Europe. Ticks Tick Borne Dis. 3, 8-13 (2012).

327 Luniak, M., Mulsow, R. \& Walasz, K. Urbanization of the European blackbird - expansion and adaptations of urban population. Urban ecological studies in Central and Eastern Europe. Proc. international symposium, Warszawa-Jablonna, 1986, 187-200 (1990)

328 McKinney, M. L. Urbanization as a major cause of biotic homogenization. Biol. Conserv. 127, 247-260, doi:http://dx.doi.org/10.1016/j.biocon.2005.09.005 (2006).

329 Fingerle, V. et al. Epidemiological aspects and molecular characterization of Borrelia burgdorferi s.l. from southern Germany with special respect to the new species Borrelia spielmanii sp. nov. Int. J. Med. Microbiol. 298, 279-290, doi:http://dx.doi.org/10.1016/j.ijmm.2007.05.002 (2008).

330 Mulder, S., Van Vliet, A. J. H., Bron, W. A., Gassner, F. \& Takken, W. High risk of tick bites in Dutch Gardens. Vector Borne Zoonotic Dis. 13, 865-871, doi:10.1089/vbz.2012.1194 (2013).

331 Rizzoli, A. et al. Ixodes ricinus and Its Transmitted Pathogens in Urban and Peri-Urban Areas in Europe: New Hazards and Relevance for Public Health. Frontiers in Public Health 2, 251, doi:10.3389/ fpubh.2014.00251 (2014). 


\section{SUMMARY}

Diseases that are transmitted by arthropod vectors from animal hosts to humans - so called zoonotic vector-borne diseases - have increased in incidence in the last decades. In North America and Europe, tick-borne pathogens cause the majority of vector-borne diseases, including Lyme borreliosis and tick-borne encephalitis. The pathogens causing these diseases are transmitted by tick species within the Ixodes ricinus complex. These are generalist ticks that have a multi-year lifecycle with three active stages, larva, nymph and adult. Each stage passively waits for a vertebrate host by questing in the vegetation. Once a host is encountered they feed on the host for several days sucking blood, after which they detach and moult to the next stage or lay eggs. Although these ticks spend the majority of their life in the vegetation, the availability of hosts is an important determinant of tick densities.

In Europe, the Sheep tick (Ixodes ricinus) is the most important vector for tick-borne pathogens. These pathogens include Borrelia burgdorferi sensu lato (s.l.), the causative agent of Lyme borreliosis, Anaplasma phagocytophilum, the causative agent of human granulocytic anaplasmosis, Borrelia miyamotoi, the causative agent of acute febrile illness and Candidatus Neoehrlichia mikurensis, the causative agent of neoehrlichiosis. There are several genospecies within the B. burgdorferi s.l. complex, among which B. afzelii, B. bavariensis, B. garinii, B. Iusitaniae, B. spielmanii, and B. valaisiana are found in questing ticks and patients in the Netherlands. All of these pathogens are maintained and amplified by vertebrate hosts. Host species differ in their ability to transmit the different pathogens (reservoir competence), as well as in their competence for ticks. Therefore, it has been hypothesized that changes in vertebrate assemblage composition can change tick-borne pathogen dynamics and thereby tick-borne 


\section{0 | Summary}

disease risk, where a decrease in host species diversity might lead to an increased disease risk, the so-called dilution effect of host species richness hypothesis.

The main objective of this thesis was to better understand the role of different vertebrate host species in maintaining $I$. ricinus populations and in infecting I. ricinus larvae with different tick-borne pathogens. In Chapter 2, I performed a systematic review on data concerning vertebrate hosts of $I$. ricinus and $B$. burgdorferi s.I. I analysed data from 66 publications and calculated the relative contribution of host species to feeding the different stages of $I$. ricinus and to infecting I. ricinus larvae with $B$. burgdorferi s.I. I found that for both mammals and birds, there was a positive correlation between host body mass and tick burden for the different stages. Nymphal burden was positively correlated with infection prevalence of hosts with $B$. burgdorferi s.l., which was again positively correlated with the average number of larvae that got infected with $B$. burgdorferi s.l. while feeding on a host. Our quantification method showed that the majority of $I$. ricinus individuals of the three stages (larva, nymph and adult) feed on only a few vertebrate host species (rodents, thrushes and deer, respectively). Based on these results, I argued that small mammal-transmitted Borrelia spp. are maintained due to the high density of their reservoir hosts, while birdtransmitted Borrelia spp. are maintained due to the high infection prevalence in their reservoir hosts. This suggests that $B$. burgdorferi s.l. genospecies evolved to be best transmitted by host species that are most often encountered, either due to high host density, or due to high encounter rates between ticks and host species (high nymphal burden). I concluded that only a few vertebrate host species, rodents, thrushes and deer, maintain I. ricinus and B. burgdorferi s.l. populations and that the increase in distribution and abundance of these host species could be the main driver behind increased tick-borne disease incidence in Europe.

From this systematic review, it became clear that there are a few host species that maintain $I$. ricinus populations and that encounter rate between ticks and hosts was an important parameter for B. burgdorferi s.l. maintenance. To study the correlations between these factors in the field, I set-up a large cross-sectional study in twenty forest plots of 1 hectare (ha) in the Netherlands. In order to study the availability to ticks of different vertebrate host species in these plots, I needed to think up a standardized method that could be used to study different vertebrate species in a quantitative way. Because the chance that a questing tick encounters an individual of a specific species depends on the number of times 
an individual of a species walks past a specific place in the forest, I decided that camera traps would be the ideal tool to study vertebrate populations because, in a way, these camera traps could be seen as questing ticks encountering vertebrate species.

Camera traps are cameras that are triggered by a passive infrared (PIR) sensor that registers a difference in thermal infrared. In my case this meant that camera traps would start to take photographs as soon as a warm-blooded animal walked past and was detected by the PIR sensor. The sensitivity of the PIR sensor is dependent on the surface temperature of the animal walking past, which is different for animals of different sizes. Bigger species are more easily detected by the PIR sensor because they omit more heat than smaller species. Therefore, bigger species are detected by the PIR sensor at larger distances than smaller species. Due to this difference, camera trap data need to be corrected in order to be able to compare the numbers of photographs taken from different animal species. This can be done by calculating the effective detection distance (EDD) of a camera trap for each species. However, established methods to estimate EDD were labour intensive. To be able to perform the fieldwork - camera trapping and tick dragging - in multiple plots on one day, I needed an easier and quicker method to estimate EDD, which is presented in Chapter 3. I found that apart from body mass, different vegetation types can also yield different EDD estimates.

I used this new method to correct photographic capture rates - as a measure of relative abundance or the availability of host species for ticks - of all animal species that were photographed using EDD estimates for the different species and vegetation types for all twenty forest plots throughout the Netherlands. In Chapter 4, I performed a deer exclusion experiment in four pairs of 0.75 -ha forest plots in a forest near Apeldoorn and used the data from this experiment and the data from the cross-sectional study to investigate the correlation between $I$. ricinus densities to the presence and relative abundance of deer. Ixodes ricinus of all stages were more abundant in plots with deer $(n=17)$ than in plots without $\operatorname{deer}(n=3)$. Where deer were present, the density of $I$. ricinus did not increase with deer abundance, with one exception: where only Roe deer were present, larval density increased with Roe deer abundance. Experimental exclusion of deer reduced nymphal density by $66 \%$ and adult density by $32 \%$ within a time frame of two years. My results suggest that deer presence rather than deer abundance drives the density of $I$. ricinus nymphs and adults. This implies that $I$. ricinus density will not necessarily decrease with a reduction in deer 


\section{2 | Summary}

density. That small exclosures ( $<1$ ha) substantially reduced I. ricinus densities suggests that fencing can be used to reduce tick-borne disease risk in areas with high recreational pressure.

From Chapter 2 it was clear that encounter rate between host individuals and ticks as measured by tick burden was an important parameter determining the importance of host species in maintaining I. ricinus populations and in determining the reservoir role of host species for tick-borne pathogens. Therefore, I set out to better understand which factors drive tick burden on rodents in Dutch forests, which is presented in Chapter 5. The I. ricinus burden on rodents can be determined by both intrinsic (e.g., body mass and sex of the host) and extrinsic (e.g., parasite density in the environment) factors. I assessed the relative importance of these factors by comparing the I. ricinus burden on rodents in the twenty plots of the cross-sectional study, and relating this to species, sex and individual body mass of the rodents as intrinsic factors, and the availability of deer to ticks, the predation risk by predators and rodent density as extrinsic factors. Average tick burden differed between species, was higher for males compared to females and increased with individual body mass. Rodents in sites with higher passage rates of deer had more ticks, while rodents in sites with higher passage rates of predators or high rodent densities had fewer ticks. I found that tick burden was most strongly correlated with extrinsic values. All relationships supported a mechanism where tick burden is determined by a combination of the number of ticks present in the environment and day range of rodents. This implies that changes in vertebrate assemblage can indirectly, via the tick burden on rodents, affect the prevalence of tick-borne pathogens.

This idea was tested in Chapter 6, where I related differences in vertebrate assemblage composition and tick burden on rodents to the DIN for rodenttransmitted tick-borne pathogens using path analysis. I tested three alternative mechanisms by which vertebrate assemblage composition can affect DIN: 1) encounter reduction between individual hosts and (infected) ticks, 2) vector augmentation by additional hosts feeding extra ticks, and 3) regulation of reservoir host populations by predators. I found similar results as in Chapter 5 for the correlation of larval burden on rodents with deer (vector augmentation), and predator abundance and rodent density (encounter reduction). DIN for $B$. afzelii, B. miyamotoi and Ca. N. mikurensis increased with larval burden on rodents. I did not find support for reservoir host regulation by predators, nor a correlation between rodent density and DIN. These results imply that changes in 
vertebrate assemblage can lead to cascading effects on rodent-transmitted tickborne disease risk, via larval burden on rodents.

Theoretical studies predict that the relative abundance of hosts that can transmit a pathogen (reservoir competent) and those that cannot transmit a pathogen (reservoir incompetent) determine tick-borne pathogen prevalence and the density of infected ticks. A change in the relative abundance of reservoircompetent hosts with host species richness, might subsequently result in the dilution or amplification of pathogen prevalence. However, empirical evidence is scarce. In Chapter 7 I investigated the correlation between pathogen prevalence in questing I. ricinus nymphs, densities of infected nymphs, the abundance of reservoir-competent and reservoir-incompetent hosts and host species richness for seven tick-borne pathogen species using data from the cross-sectional study. Infection prevalence in nymphs for six pathogen species, and density of infected nymphs for three pathogen species increased with abundance of reservoircompetent hosts (amplification effect), and reservoir-competent host abundance rose with host species richness in my study plots. Nymphal infection prevalence decreased with reservoir-incompetent host abundance for three pathogens (dilution effect). To test if correlations found at this small spatial scale resulted in large scale differences, I tested for a correlation of mammal-host species richness with Lyme borreliosis incidence for 17 countries in Western Europe. At this scale, Lyme borreliosis incidence increased with mammal-host species richness. These results are in disagreement with a dilution effect of host species richness for tick-borne pathogens in Europe. Rather, the relative densities of reservoir-competent and reservoir-incompetent host species are the most important parameters determining tick-borne disease risk.

These results are discussed and synthesized in Chapter 8, where I discuss my findings on tick-borne pathogens in the Netherlands, and relate these to previous studies on the relationship between vertebrate hosts and tick-borne pathogen prevalence. Furthermore, I present a mathematical model in which the importance of spatial behaviour of hosts for tick-borne pathogens is shown and I proclaim the need for the integration of the field of behavioural ecology into disease ecology to better understand the effect of changes in vertebrate assemblages on pathogen prevalence and ultimately, disease risk. The data presented in this thesis show that it is not host diversity but the presence, abundance and behaviour of specific host species that drives tick-borne pathogen dynamics (identity effect). Vertebrate species change their behaviour in the presence or 


\section{4 | Summary}

absence of predators and competitors. I show that this, theoretically, can have a major influence on the density of infected nymphs in the vegetation. Therefore, behavioural changes of reservoir-competent hosts should be taken into account when modelling the effect of changes in vertebrate assemblage composition on tick-borne disease risk.

The behaviour of vertebrate species in Europe is changing, as multiple species have adapted to human-dominated and fragmented landscapes. The adaptation of small mammals, thrushes and deer to fragmented landscapes might be one of the driving factors behind the increase in tick-borne disease incidence in Europe. A further adaptation of important host species to urbanized landscapes might be expected as these are the safest areas for vertebrate species trying to avoid predation. This might result in an increase in population density of reservoircompetent host species in urban areas with a corresponding increase in tickborne pathogen prevalence and therefore, tick-borne disease risk.

Concluding, our world is changing and as a consequence vertebrate assemblages are also changing. This may lead to changes in I. ricinus density and infection prevalence with tick-borne pathogens. From the studies presented in this thesis I conclude that the abundance and behaviour of several host species (e.g., Bank vole, Blackbird, Red deer, Red fox, Roe deer, and Wood mouse) determines tick-borne disease risk. Therefore, studying the drivers of animal abundance and behaviour related to ticks and pathogens will be the next step in better understanding and describing tick-borne disease risk. The ecology of tickborne pathogens is very complex and targeting vertebrate hosts for intervention strategies will be both inefficient and costly due to the intricate interplay between multiple vertebrate host species. Therefore, I conclude that prevention of tick bites is the best way to reduce tick-borne disease incidence. 
Summary | 195 
196 


\section{SAMENVATTING}

Ziektes die door teken worden overgedragen vormen in Nederland een steeds groter probleem. Jaarlijks worden er bijvoorbeeld ongeveer 25.000 mensen besmet met Borrelia burgdorferi s.l., het bacteriecomplex dat de ziekte van Lyme veroorzaakt. Er zijn echter meer ziekteverwekkers die door teken kunnen worden overgedragen, zoals het virus dat teken-encefalitis veroorzaakt. De meest voorkomende tekensoort in Nederland is de schapenteek Ixodes ricinus. Deze tekensoort heeft drie levensstadia: de larve, nimf en adult, die passief in de vegetatie wachten op een gewervelde gastheer. leder stadium heeft bloed nodig van een gastheer om te overleven of om eitjes te kunnen produceren. Omdat de teken wachten op een gastheer is de kans dat ze een gastheer vinden afhankelijk van de dichtheid van de verschillende soorten gastheren. Hierdoor is de beschikbaarheid van gastheren een belangrijke factor die de tekendichtheid bepaald.

Tijdens die ene bloedmaaltijd per stadium kunnen teken besmet raken met bacteriën die door de gastheer worden overgedragen. Voorbeelden zijn Borrelia burgdorferi sensu lato (s.l.), het bacterie-complex dat de ziekte van Lyme veroorzaakt, Anaplasma phagocytophilum, de bacterie die Humane Granulocytaire Anaplasmose veroorzaakt, en andere ziekteverwekkers zoals Borrelia miyamotoi en Candidatus Neoehrlichia mikurensis. In Nederland zijn er verschillende geno-soorten binnen het Borrelia burgdorferi s.l. complex gevonden in teken en patiënten, namelijk: B. afzelii, B. bavariensis, b. garinii, B. Iusitaniae, $B$. spielmanii en $B$. valaisiana. Al deze ziekteverwekkers worden onderhouden door gewervelde dieren. Er zijn verschillende soorten zoogdieren en vogels die als gastheer kunnen dienen voor de schapenteek, en deze gastheersoorten verschillen in hoe goed ze verschillende ziekteverwekkers kunnen overdragen. 


\section{8 | Samenvatting}

Ook verschillen de gastheersoorten in hoeveel teken ze gemiddeld voeden. Hierdoor heeft de soortensamenstelling van de gastheren in een gebied invloed op hoeveel schapenteken er in de vegetatie gevonden worden en welk percentage van die teken besmet is met de verschillende bacterie soorten. Dat effect van de gastheergemeenschap heeft onderzoekers in Noord-Amerika geïnspireerd tot het stellen van het zogenaamde 'verdunningseffect' waarbij een toename in soortenrijkdom van gastheren zou leiden tot een afname in ziekterisico, kortweg, biodiversiteit beschermt tegen de kans op ziekte.

Het voornaamste doel van dit proefschrift was om een beter beeld te krijgen van de rol die verschillende diersoorten spelen in het onderhouden van schapenteekpopulaties en in het besmetten van de schapenteek met verschillende teken-overdraagbare ziekteverwekkers. In Hoofdstuk 2 presenteer ik een 'systematische review' waarin ik data van 66 studies heb gebruikt om te kijken welke gastheersoorten het belangrijkst zijn in het voeden van de verschillende stadia van de schapenteek en in het besmetten van het larvale stadium van de schapenteek met B. burgdorferi s.l. Ik vond dat het gewicht van een diersoort positief gecorreleerd was met het aantal teken dat die soort gemiddeld bij zich droeg. De gemiddelde besmettingsgraad van een diersoort nam toe met het gemiddeld aantal nimfen, vervolgens nam het gemiddeld aantal larven dat door een gastheersoort besmet wordt met B. burgdorferi s.l. toe met de gemiddelde besmettingsgraad van de gastheer. Uit een simpel model, gebaseerd op de gemiddelde tekenlast van een diersoort en hun dichtheden, bleek dat kleine knaagdieren zoals muizen en woelmuizen, lijsterachtigen en herten waarschijnlijk de belangrijkste gastheren zijn voor, respectievelijk, de larven, nimfen en adulten. Er zijn dus maar een paar soorten gastheren nodig om een tekenpopulatie in stand te kunnen houden. Ook bleek dat, in de gemiddelde Europese situatie, muizen de belangrijkste gastheergroep zijn die met $B$. burgdorferi besmette nimfen voortbrengt. Gebaseerd op mijn bevindingen verwachtte ik dat $B$. burgdorferi s.l. twee strategieën kan hebben om te overleven. Deze strategieën zijn afhankelijk van een hoge infectiegraad in teken dankzij een hoge gastheerdichtheid (bij door muizen overgedragen Borrelia soorten) of afhankelijk van een hoge infectiegraad in gastheren dankzij een hoge tekenlast (bij door vogels overgedragen Borrelia soorten). Dit suggereert dat B. burgdorferi s.l. geno-soorten geëvolueerd zijn om overgedragen te worden door de gastheren die ze vaak tegen komen. De aantallen en verspreiding van de belangrijkste gastheren voor de schapenteek (muizen, lijsterachtigen en herten) zijn in de 
laatste decennia alle drie toegenomen wat een verklaring zou kunnen zijn voor de toename in het aantal patiënten met door teken overgedragen ziektes.

Uit de systematische review bleek dat er maar een paar gastheersoorten zijn die belangrijk zijn voor het onderhouden van schapenteekpopulaties en dat de trefkans tussen gastheren en teken een belangrijke factor is die mogelijk de infectiegraad in teken met B. burgdorferi s.l. bepaalt. Om uit te zoeken of dit ook overeenkomt met de aantallen teken en hun besmettingsgraad in het veld, heb ik een grote veldstudie uitgevoerd waarin ik metingen heb gedaan in twintig bosplots van 1 hectare (ha) verspreid over Nederland. Voor het meten van de beschikbaarheid van verschillende gastheersoorten voor teken had ik een gestandaardiseerde methode nodig waarmee ik verschillende diersoorten op een kwalitatieve manier kon onderzoeken. Omdat de kans dat een teek die in de vegetatie zit te wachten ook daadwerkelijk een gastheer tegenkomt afhankelijk is van hoe vaak er een gastheer passeert, besloot ik om cameravallen te gebruiken om de gastheerpopulatie te onderzoeken. Een cameraval kon immers meten hoeveel dieren er per tijdseenheid passeerden, en op die manier dienst doen als een surrogaat teek die zat te wachten op een gastheer.

Cameravallen zijn camera's die worden geactiveerd door een passieve infrarood (PIR) sensor. Een PIR sensor meet een verschil in thermaal infrarood. In mijn geval betekende dit dat de cameravallen foto's gingen maken zodra er een warmbloedig dier langs liep dat werd gedetecteerd door de PIR sensor. De gevoeligheid van de PIR sensor is afhankelijk van de oppervlaktetemperatuur van het dier dat langsloopt, wat weer gerelateerd is aan de grootte van de diersoort. Grotere soorten worden gemakkelijker door de PIR sensor gedetecteerd omdat ze meer warmte uitstralen dan kleinere soorten. Vanwege dit verschil moeten cameravaldata gecorrigeerd worden om de aantallen passages van verschillende diersoorten met elkaar te kunnen vergelijken. Dit kan door de effectieve detectie afstand (EDA) van de cameraval voor iedere diersoort te berekenen. Bestaande methodes voor het bepalen van de EDA waren erg arbeidsintensief. Dus om mijn veldwerk uit te kunnen voeren, waarbij ik zowel cameravallen moest plaatsen als teken moest slepen in meerdere plots per dag, had ik een snellere en makkelijkere manier nodig om de EDA te kunnen bepalen. Ik presenteer deze methode in Hoofdstuk 3. Ik vond dat, naast lichaamsgewicht, de dichtheid van de vegetatie ook een effect had op de EDA.

Ik heb mijn nieuwe methode gebruikt om de aantallen passages - als maat voor de beschikbaarheid van een diersoort voor teken - te corrigeren 


\section{0 | Samenvatting}

gebaseerd op EDA's die bepaald waren per diersoort en per vegetatiesoort. Die gecorrigeerde aantallen passages heb ik gebruikt om de beschikbaarheid van alle verschillende soorten gastheren (zoogdieren en vogels) voor teken te bepalen in de twintig bosplots door heel Nederland. Voor Hoofdstuk 4 heb ik daarnaast ook een experiment uitgevoerd in het Achterpark van Kroondomein het Loo bij Apeldoorn. Voor dit experiment hebben we 4 bosplots van $\pm 0,75$ ha uitgerasterd met een 2,2 $\mathrm{m}$ hoog hek en deze vergeleken met 4 bosplots zonder hek die $100 \mathrm{~m}$ verderop lagen. Dit experiment, en de data uit de twintig bosplots verspreid over heel Nederland gebruikte ik om de correlatie tussen de dichtheid van de schapentekenpopulatie en de beschikbaarheid van herten voor teken te bestuderen. Alle drie de stadie van de schapenteek hadden significant hogere dichtheden in de 17 plots waar herten voorkwamen vergeleken met de 3 plots zonder herten. Waar herten aanwezig waren was er geen correlatie tussen de het aantal schapenteken en de beschikbaarheid van herten met één uitzondering: waar alleen reeën aanwezig waren was er een positieve correlatie tussen het aantal reeën en het aantal larven in de vegetatie. Het experimenteel uitsluiten van herten resulteerde binnen twee jaar in een reductie van het aantal nimfen met $66 \%$ en van het aantal adulten met $32 \%$. Hieruit concludeer ik dat de aanwezigheid van herten essentieel is voor de schapenteek, maar dat meer herten niet noodzakelijk leidt tot meer teken. De afrastering van kleine gebieden $(<1 \mathrm{ha}$ ) leidde tot een significante afname in de aantallen teken in de vegetatie. Daarom kan het afrasteren van gebieden een goede methode zijn om het risico op teken-overgedragen ziektes te verlagen op plekken met lokaal veel recreanten.

In Hoofdstuk 2 bleek dat de trefkans tussen individuele gastheren en schapenteken, gemeten als de tekenlast van een gastheer, een belangrijke factor was die bepaalde hoe belangrijk een gastheersoort was voor de instandhouding van schapenteekpopulaties. Daarnaast leek die tekenlast ook te bepalen hoe goed een soort $B$. burgdorferi s.l. over kon brengen naar voedende larven. Daarom was ik benieuwd welke factoren het aantal teken op een muis in Nederlandse bossen bepaalde. Dit heb ik uitgezocht in Hoofdstuk 5. De schapentekenlast van muizen wordt bepaald door zowel intrinsieke factoren (bijvoorbeeld lichaamsgewicht en geslacht van de gastheer) als extrinsieke factoren (bijvoorbeeld de hoeveelheid teken in de omgeving). Ik heb getracht om te bepalen welke van deze twee typen factoren de meeste invloed heeft op de aantallen schapenteken op een muis. Dit deed ik door de aantallen schapenteken op muizen te meten in de twintig bosplots in heel Nederland en deze aantallen te correleren aan de soort, het geslacht en het 
lichaamsgewicht van iedere individuele muis (als intrinsieke factoren) en aan de beschikbaarheid van herten voor teken, het predatierisico voor muizen, bepaald door het gemiddeld aantal passages van roofdieren, en de muizendichtheid (als extrinsieke factoren). De gemiddelde tekenlast van een muis was verschillend voor verschillende muizensoorten (aardmuis, bosmuis en rosse woelmuis), was hoger voor mannetjes dan voor vrouwtjes en nam toe met het lichaamsgewicht van de muis. Muizen in gebieden met meer herten hadden meer teken, terwijl muizen in gebieden met meer predatoren of een hogere muizendichtheid juist een lagere tekenlast hadden. Ik vond dat de tekenlast van muizen het sterkst gecorreleerd was aan extrinsieke factoren, zoals het aantal herten en het aantal roofdieren. Alle gevonden relaties ondersteunen een mechanisme waarbij de tekenlast van een muis wordt bepaald door twee factoren: 1) het aantal teken in de vegetatie en 2) de afstand die een muis gemiddeld per dag aflegt. Dit impliceert dat veranderingen in de soortsamenstelling van een gemeenschap van gewervelde dieren via de tekenlast van muizen een indirect effect kan hebben op het ziekterisico op door teken overgedragen ziekteverwekkers.

Dit idee heb ik getest in Hoofdstuk 6 waar ik verschillen in de samenstelling van de gastheergemeenschap en de tekenlast van muizen heb vergeleken met de dichtheid aan geïnfecteerde nimfen die besmet waren met door muizen overgedragen teken-overdraagbare ziekteverwekkers. Met een pad-analyse testte ik drie verschillende mechanismes waarmee de gastheergemeenschap invloed kan hebben op het aantal besmette nimfen in de vegetatie, namelijk: 1) trefkansreductie tussen individuele gastheren en (geïnfecteerde) teken, 2) tekentoename dankzij de aanwezigheid van gastheren die extra teken voeden waardoor de tekenpopulatie groter wordt en 3) regulatie van competente gastheren door predatoren. Ik vond vergelijkbare resultaten als in Hoofdstuk 5 wat betreft de correlaties tussen de larvenlast van muizen en herten (tekentoename) en de correlatie tussen de larvenlast van muizen en roofdieren (trefkansreductie). Het aantal nimfen in de vegetatie dat geïnfecteerd was met B. afzelii, B. miyamotoi en Ca. N. mikurensis nam toe met de larvenlast van muizen, maar niet met muizendichtheid. Ook was muizendichtheid niet gecorreleerd met het aantal roofdieren in een plot. Kortom, het is de tekenlast van een muis, en niet de muizendichtheid die voor een belangrijk deel bepaalt hoeveel teken in de vegetatie besmet zijn met door muizen overgedragen tekenoverdraagbare ziekteverwekkers, en die tekenlast is voornamelijk afhankelijk van het aantal herten (positief) en het aantal roofdieren en muizen (negatief) 


\section{2 | Samenvatting}

dat in een gebied voorkomt. Deze resultaten suggereren dat veranderingen in de gastheergemeenschap een cascade-effect kunnen hebben op het risico op door muizen overgedragen teken-overdraagbare aandoeningen via een effect op de larvenlast van muizen.

Modelstudies hebben voorspeld dat de infectiegraad van ziekteverwekkers in teken en de dichtheid van geïnfecteerde teken bepaald worden door de relatieve dichtheden van gastheren die een ziekteverwekker kunnen overdragen (reservoir competent) en van gastheren die een ziekteverwekker niet kunnen overdagen (reservoir incompetent). Als een verandering in soortenrijkdom van de gastheren gepaard gaat met een verandering in de relatieve dichtheid van reservoircompetente gastheren kan dit leiden tot een toename of een afname van de infectiegraad van een ziekteverwekker. Empirisch bewijs hiervoor is echter schaars. In Hoofdstuk 7 heb ik onderzoek gedaan naar de infectiegraad van zeven ziekteverwekkers in schapenteeknimfen, het aantal nimfen in de vegetatie dat geïnfecteerd was met die ziekteverwekkers, de relatieve beschikbaarheid van reservoircompetente en reservoirincompetente gastheren en de soortenrijkdom van gastheren in de twintig bosplots van mijn onderzoek. De infectiegraad van zes ziekteverwekkers en het aantal nimfen in de vegetatie geïnfecteerd met drie ziekteverwekkers namen toe met de relatieve beschikbaarheid van competente gastheren (een zogenoemd vermenigvuldigingseffect). De infectiegraad van drie ziekteverwekkers nam af met de relatieve beschikbaarheid van incompetente gastheren (het reeds genoemde verdunningseffect). Daarnaast nam in mijn onderzoeksgebieden de relatieve beschikbaarheid van competente gastheren toe met de soortenrijkdom van de gastheergemeenschap. Dit in tegenstelling tot onderzoek uit de Verenigde Staten waar deze correlatie negatief was. Ik wilde weten of deze vondsten op een kleine ruimtelijke schaal effect zouden kunnen hebben op het ziekterisico op een grotere ruimtelijke schaal. Om dit te testen heb ik gekeken naar de correlatie tussen zoogdierrijkdom en het aantal mensen dat jaarlijks gediagnosticeerd wordt met de ziekte van Lyme in 17 landen in Europa. Op deze ruimtelijke schaal bleek het aantal mensen dat ziek wordt positief gecorreleerd met de zoogdierrijkdom in een land. Deze vondsten komen niet overeen met de voorspellingen van de 'verdunningseffect door soortenrijkdom' hypothese die gebaseerd is op vondsten uit Noord-Amerika. In plaats daarvan is de identiteit van de gastheer erg belangrijk en bepaald de combinatie aan competente en niet competente gastheren het ziekte risico voor teken-overdraagbare aandoeningen. 
Ik bediscussieer de resultaten uit alle voorgaande hoofdstukken in Hoofdstuk 8. In dit hoofdstuk bespreek ik mijn bevindingen op het gebied van tekenoverdraagbare aandoeningen in Nederland en vergelijk ik deze met voorgaande studies naar de relatie tussen gastheren en de prevalentie van door tekenoverdraagbare ziekteverwekkers. Daarnaast presenteer ik een wiskundig model waarmee ik laat zien dat het ruimtelijke gedrag van een gastheer een belangrijke factor is die bepaalt hoeveel teken er besmet zijn met een bepaalde ziekteverwekker. Daarom denk ik dat het onderzoek naar gedragsecologie en het onderzoek naar ziekte-ecologie gecombineerd zouden moeten worden om beter te begrijpen welke mechanismes een rol spelen in het bepalen van de infectiegraad van ziekteverwekkers, oftewel het ziekterisico. Mijn onderzoek laat zien dat het niet de soortenrijkdom van gastheren is, maar de aanwezigheid, dichtheid en het gedrag van specifieke gastheersoorten die bepalend zijn voor het ziekterisico op door teken overgedragen aandoeningen (een zogenoemd identiteitseffect). Die specifieke gastheren veranderen hun gedrag als reactie op de aanwezigheid van andere dieren zoals concurrenten of roofdieren. Die veranderingen in gedrag kunnen, in ieder geval theoretisch, een groot effect hebben op het aantal geïnfecteerde teken in een gebied. Daarom is het belangrijk om gedragsveranderingen van competente gastheren in acht te nemen in modellen die proberen om het effect van gastheergemeenschappen op ziekterisico te voorspellen.

Zoogdieren en vogels in Europa veranderen momenteel hun gedrag, als reactie op het gefragmenteerde landschap dat gedomineerd wordt door bevolkte gebieden. Muizen, lijsterachtigen en herten hebben zich aangepast aan het leven in een gefragmenteerd landschap wat één van de belangrijkste factoren zou kunnen zijn voor de toename in het aantal mensen dat ziek wordt dankzij een door teken overgedragen ziekteverwekker. Deze gastheren kunnen zich nog verder aanpassen aan leven in een stedelijke omgeving, zeker als de terugkeer van predatoren het predatierisico buiten steden hoger maakt. Dit zou kunnen zorgen voor een verhoogd aantal reservoircompetente gastheersoorten in stedelijke omgevingen dat kan leiden tot een verhoogd ziekterisico in stadsparken en tuinen.

Onze wereld verandert en het gevolg is dat de gemeenschappen van dieren ook veranderen. Dit kan er toe leiden dat dichtheden van schapenteken en de infectiegraad van ziekteverwekkers in teken gaan veranderen. Uit mijn onderzoek blijkt dat de dichtheden en het gedrag van een paar gastheersoorten (o.a. 


\section{4 | Samenvatting}

bosmuis, edelhert, merel, ree, vos en rosse woelmuis) het ziekterisico op door teken-overgedragen ziekteverwekkers bepalen. Daarom is het belangrijk om een beter beeld te krijgen van wat de dichtheden en het gedrag van deze soorten bepaalt, zeker in relatie tot teken. De ecologie van door teken overgedragen ziekteverwekkers is erg complex, wat het bestrijden van teken door het aanpakken van gastheren erg inefficiënt en kostbaar zal maken. Daarom denk ik dat het voorkomen van tekenbeten door middel van de juiste kleding en goede controle de beste manier is om het aantal mensen dat ziek wordt te verlagen.

\section{Implicaties voor natuurbeheerders}

Tijdens mijn onderzoek heb ik veel gesproken met de natuurbeheerders die de gebieden beheren waar ik mijn onderzoek heb gedaan. In deze paragraaf zal ik proberen om mijn onderzoeksresultaten te vertalen naar enkele aanbevelingen voor natuurbeheerders in Nederland. Uit mijn onderzoek blijkt dat de aanwezigheid van herten de voornaamste factor is die bepaalt hoeveel teken er in een gebied voorkomen. Zodra enkele herten aanwezig zijn is dit voldoende om een tekenpopulatie in stand te houden en het maakt niet uit of dit damherten, edelherten of reeën zijn (Hoofdstuk 4). Het aantal larven in de vegetatie, en daardoor het aantal larven dat beschikbaar is voor muizen of merels, neemt wel toe met het aantal herten (Hoofdstukken 5 \& 6). Echter, het aantal herten is niet de enige factor die invloed heeft op het aantal larven op een muis. De muizendichtheid en het aantal roofdieren waren net zo belangrijk (Hoofdstukken $5 \& 6$ ). Daarom zal het beheren van de hertenpopulatie niet altijd een gewenst effect geven op de tekenpopulatie, maar hangt dit ook af van de muizen- en roofdierpopulatie in het gebied. Het lokaal uitrasteren van herten op plekken waar veel gerecreëerd wordt, zoals picknickplekken, speelweides of natuurcampings, zou echter wel resulteren in een significante verlaging van de tekendichtheid op deze plekken (Hoofdstuk 4). Of dit ook gepaard gaat met een verlaging van het ziekterisico is echter maar de vraag, omdat herten incompetente gastheren zijn voor veel ziekteverwekkers die door teken worden overgedragen, waardoor de infectiegraad met deze ziekteverwekkers in teken lager is in gebieden met veel herten (Hoofdstuk 7). Hierbij komt het menselijk gedrag ook om de hoek kijken. Is het risico hoger in een gebied met 10 teken waarvan er 5 besmet zijn (50\% besmettingsgraad; hypothetisch in een uitgerasterd gebied waar wel muizen en merels voorkomen) ten opzichte van een gebied met 100 teken waarvan er 
10 besmet zijn ( $10 \%$ besmettingsgraad; hypothetisch in de omgeving van de afrastering waar wel herten rondlopen)? Het aantal besmette teken is lager in de eerste situatie, dus de kans om een besmette teek tegen te komen is lager. Maar de kans om überhaupt een teek tegen te komen is ook lager, en daardoor zullen mensen zich mogelijk veiliger voelen en niet op teken controleren, terwijl in het tweede gebied al tijdens de wandeling een teek wordt opgemerkt en deze mensen dus met zekerheid thuis gaan controleren op teken (als ze goed zijn voorgelicht en verstandig zijn). Kortom, het beschrijven welke factoren zorgen voor veel besmette teken in de vegetatie, iets wat ik aan de hand van de resultaten uit mijn proefschrift wel durf te doen, hoeft niet persé overeen te komen met het daadwerkelijke risico voor bezoekers van een gebied.

Daarnaast hebben alle diersoorten ook invloed op elkaar en elkaars gedrag. Hierdoor zal één diersoort beheren om het ziekterisico te verminderen ook een invloed hebben op de dichtheden en het gedrag van andere diersoorten, wat niet goed te voorspellen is. Daarom is mijn advies om geen beheer toe te passen gebaseerd op het verminderen van dierenpopulaties, maar in plaats daarvan lokaal goede voorlichting te geven op locaties waar het risico op het oplopen van een besmette teek het hoogst is. Dit is in bosvakken met regelmatig een hert of een ree, weinig roofdieren en veel muizen en merels. Als deze gebieden buiten de wandelroutes worden gehouden en er voorlichtingsborden of -posters geplaatst worden bij de parkeerplaats met daarop duidelijke foto's van hoe klein teken zijn en waar mensen op moeten letten bij het controleren en daarna, denk ik dat het aantal patiënten met door teken overgedragen aandoeningen niet verder hoeft te stijgen en misschien zelfs af kan nemen. 
206 


\section{AfFiLIATION OF CO-AUTHORS}

Patrick A. Jansen, Herbert H.T. Prins, Sipke E. van Wieren, H. Jan Wijnen:

Resource Ecology Group, Wageningen University, Wageningen, the Netherlands

Willem Takken:

Laboratory of Entomology, Wageningen University, Wageningen, the Netherlands

E. Claudia Coipan, Manoj Fonville, Hein Sprong:

Centre for Infectious Disease Control Netherlands, National Institute for Public Health and the Environment, Bilthoven, the Netherlands

J. Marcus Rowcliffe:

Institute of Zoology, Zoological Society of London, London, United Kingdom 
208 


\section{ACKNOWLEDGEMENTS}

If you would have told me five years ago that I would obtain my PhD by studying ticks, I would not have believed you. Just before I started the work that resulted in this PhD thesis I was convinced that population genetics was going to be my future. So I am very happy that I met this wonderful professor called Herbert Prins, who saw the potential in me to become a disease ecologist and asked me to work on the ecology of Lyme disease. It was only after one year into my PhD that I realized that he was right and that disease ecology combines everything that I find interesting, especially mammals, but also arthropods, evolution and genetics. Thank you Herbert for putting me on this path, which I am now so happy to be on! Also, although you were away a lot, always in some tropical or otherwise marvellous place, you were there for me when I really needed it most. Thank you!

I was a really lucky guy if you consider supervision. First of all, if I needed to know anything about Dutch forests or large mammals, I could walk to the other side of Lumen and ask Sip. Sip, you are one of the few real field ecologists left in the Netherlands if it comes to animal ecology (and especially mammalogy). Although we only spent little time together in the field, I really learned a lot from you, especially if it comes down to 'reading' herbivory signs. For me it feels like we explored the terrain of tick ecology together and by doing fieldwork, reading and talking, we sharpened our view on the ecology of Ixodes ricinus. You never stopped being critical about my ideas and work, which always improved whatever I did. Thank you for that!

Secondly, if I needed to know anything about camera traps, methodology or statistics, I could walk to the room next door and ask Patrick. Patrick, I am very grateful for all the hours that we spent cooking up great new ideas, figuring out the best way to analyse those hierarchical datasets, and refining texts. Your way 


\section{0 | Acknowledgements}

of approaching science and manuscripts has really shaped my writing and way of thinking. I am very happy that you were one of my mentors and I hope that we will be able to come up with some cool camera trapping project in the future!

Last but not least, if I needed to know anything about the nasty bacteria that reside in ticks, I could call to the RIVM and ask Hein. Hein, although officially you were not one of my supervisors, for me, you really are one of my mentors. I really appreciate your enthusiasm, which was very contagious. Also, thank you for your confidence in me and providing me with the extra funding to be able to perform an extra project next to finishing my thesis. Your confidence in me has given me the extra self-confidence that I needed to finish this thesis.

Of course I was not the only one in the beautiful 'Shooting the messenger' project. First of all a big thank you to Gilian and Claudia for being my partners in crime, it was always fun to discuss ideas or new data with you and I am super happy that the combination of our three theses really made a big contribution to our understanding of the ecology of ticks and tick-borne diseases. Willem, Sip and Hein, as coordinators of the project you made sure that everything went smoothly and things were arranged in time. Thank you for that! And thank you Marieta for living up our meetings every now and then!

This thesis would not have been possible without a big amount of field work. I drove $>22,000 \mathrm{~km}$ around the Netherlands, caught 17,401 ticks (not including $>10,000$ larvae), counted $>10,000$ ticks on 661 rodents and got approximately 100 tick bites. All that work would not have been possible without the help of Frans. Frans, thank you very much for helping me with the tick dragging during two field seasons! I would also like to thank all other colleagues and students that helped during the field work presented in this thesis: Helen, Herman, Jan, Mikhail, Pieter-Bas, Rutger, San, Wouter and Zheng, thank you very much! All that field work would not have been possible without the approval of all the managers of the forests in which I did my research. Special thanks go out to Bert Biemans, Rogier Dijkstra, Arthur Ebregt, Karel van der Heijden, Henk Hofman, Hans Hoogewerf, Chris van den Hoven, Marcel Geurts, Leon Kelder, Ruud Luntz, Jan Rots, Koen Rotteveel, Jaap Rouwenhorst, Han ten Seldam, Vincent van der Spek, Hugo Spitzen, and Bertil Zoer for providing access to the nature reserves.

Collecting $>16,000$ nymphs is already a lot of work, but testing all these nymphs for different tick-borne pathogens takes even longer. I am very grateful to Claudia and Manoj for doing the enormous amount of lab work of which the results are presented in this thesis, thank you! Special thanks also go out to 
Marco Visser and Gerrit Gort for getting me acquainted with the wonderful world of generalized linear mixed models.

Doing all that field work, analysing and writing would not have been half as fun without the wonderful colleagues at REG, (and a little bit of FEM and PEN). Audri, Benson, Bernardo, Daniel, David, Dorit, Edson, Eduardo, Erlinda, Faith, Frank, Fred, Henjo, Herman, Ignas, Iris, Jasper, Jente, Jeroen, Jing, Joost, Juan, Jusper, Kevin, Kyle, Lennart, Mart, Martijn, Mikhail, Milena, Mina, Ntuthuko, Peter, Peter, Pim, Priya, Rafael, Richard, Richard, Rob, Ron, Shyam, Sintayehu, Tibor, Vincent, Yanjie, Yin, Yingying, Yorick, Yussuf, Zhang and Zheng thank you for the wonderful years in Lumen. Special thanks go out to Helen, thank you for being my office mate all these years. I really enjoyed our conversations about ticks, tickborne diseases and all other random things that passed the office over all these years, including social dancing and the differences between balfolk and salsa. I really enjoyed sharing my office with you, and of course, thank you for being my paranymph (pun intended)! A thesis cannot be written without the support of people that take care of office supplies, expenses and other paperwork. A big thank you for Gerda, Joke and Patricia for taking care of me at REG, and thank you Angelique for taking care of me at Entomology.

I really enjoy teaching, so I was very happy that I had the opportunity to supervise several MSc students and two BSc students during their thesis. Annemieke, Arjan, Astrid, Bob, Esther, Jan, Marije, Marijn, Pieter-Bas, Rutger, San, Sander, and Wiegert, thank you for being my students. Although you might not realize it, I think I might have learned more from you than you did from me. Supervising each and every one of you was another experience that taught me a lot. Thank you for those lessons.

I would not have been the person I am now without the guidance and company of some great people. First of all, Sim, Hugh, Jasja and Gerard, thank you for your guidance during the many hours spent behind a table dissecting all kinds of Mustelids and thank you for always being interested in my work, even when I stopped working on Mustelids. Also, my PhD journey would have been very different if I would not have been a board member of the Small Mustelid working group. Jeroen, Erwin, Fokko, Johann, Carola and Matthijs, thank you very much for all the fun hours spent in the field, and all the hard work trying to organize and arrange things for the working group! Last, but not least, a big thank you to René, Maurice, Hans, Bart, and Wouter for being such a fun and enthusiastic team validating all the mammal sightings at waarneming.nl. 


\section{2 | Acknowledgements}

Luckily, there is a life outside of work during your PhD! First of all a big thank you to Rutger, Thijs, Joachim, Paul and Tim for our regular Bier \& Pizza evenings in which I could escape from ticks and mice and talk about birds. Although the last couple of times we talked significantly more about other stuff (including work), maybe we are finally becoming mature..?! I just hope that we will keep meeting regularly and keep talking about birds! Also, a big thank you to all other guys from the 4 bier chat: Jurrien, Daan, Jacob, Pieter, Jan, Rens, Ruben, and Thijs.

At the start of my PhD, I spent a large part of my free time taking photos, mainly in nature, and I was a board member of 'Natuurfoto Rivierenland'. Thanks to Ben, Bendiks, Dick, Jan, Harry, Jolanda and all the others for the nice evenings and excursions we had, talking about photography and actually taking photos. Those moments were really important for me to escape my work.

Vincent, David, and Elvira, I know that we have spoken far less than I would have liked since I started my $\mathrm{PhD}$ and you all moved out of Wageningen. Therefore, those moments when we were together were extra special! Especially the time around Christmas when we were all in the Netherlands were great, including an epic LAN party and some crazy movie nights. David, I am really sorry that we are so bad at keeping in touch since you moved to Vancouver. Seeing you every week was really important for me during the start of my $\mathrm{PhD}$, just to talk about random things, see some terrible series and movies, eating unhealthy food and drink too much beer (but that was OK, because we had spent an hour on the squash track!). Spending two weeks with you and Jolanda on Vancouver Island and in Vancouver were two of the greatest weeks of my life. I really hope that we can keep in touch a little bit better, and that we can have many more great holidays together!

The most important hobby that I re-discovered during my $\mathrm{PhD}$ was making music. Without music, I would not have been able to finish my PhD, or do anything at all at the moment. A big thank you to the guys from Andoorn: Bram, Jasper, Wouter and Kris. I really could not have done this without you guys! I am really proud of what we accomplished together in the last couple of years, and I am happy that we can show that with our beautiful CD 'Van Vandaag, Voor Morgen'. Kris, I have called you my musical soulmate, but you are more than that. Our discussions about science, life, music, and many other things have really shaped me during the last couple of years. I still think it is crazy that we decided to play some music together in 2007 which resulted in Andoorn, and that we did exactly the same again in the Autumn of 2015 , which resulted in a performance 
at Boombalfestival and a beautiful CD with Nubia only a year later. I am very happy that we could escape from our PhD work together, dancing, making music, writing texts, drinking beer and getting goose bumps when we really went up in the music. This PhD thesis would not have been what it is without you, so I am really happy and thankful that you are my paranymph. I just hope that we can keep playing music together for the rest of our lives!

$\mathrm{Pa}$ en mam, dank voor alle steun en vertrouwen die ik al die jaren van jullie heb gekregen. Jullie hebben mij altijd vertrouwd om de keuzes te maken die voor mij het beste waren en dat heeft nu tot dit proefschrift geleidt. Die was er niet geweest zonder jullie! Pa, mam en Nik. Ik zie jullie veel minder dan ik graag zou willen, al sinds ik in Wageningen woon. (Te) veel hobbies zorgen er voor dat ik veel minder bij jullie langs kan komen dan ik eigenlijk zou willen. Bellen vind ik ook al niks, kortom ik zie jullie te weinig. Maar dat neemt niet weg dat ik heel veel van jullie hou! Dankjulliewel voor de eindeloze support. En als laatste natuurlijk Jolanda. Lieve schat, dankjewel voor alle steun die je me de laatste jaren hebt gegeven. Altijd als iets niet lekker ging, of ik niet uit een analyse kwam kon ik bij jou terecht om m'n frustratie te uiten. Maar samen kwamen we er altijd uit. Zonder jou was deze thesis er niet geweest. Dankjewel voor de vele mooie jaren en ik hoop dat er nog veel zullen volgen. Dikke kus! 


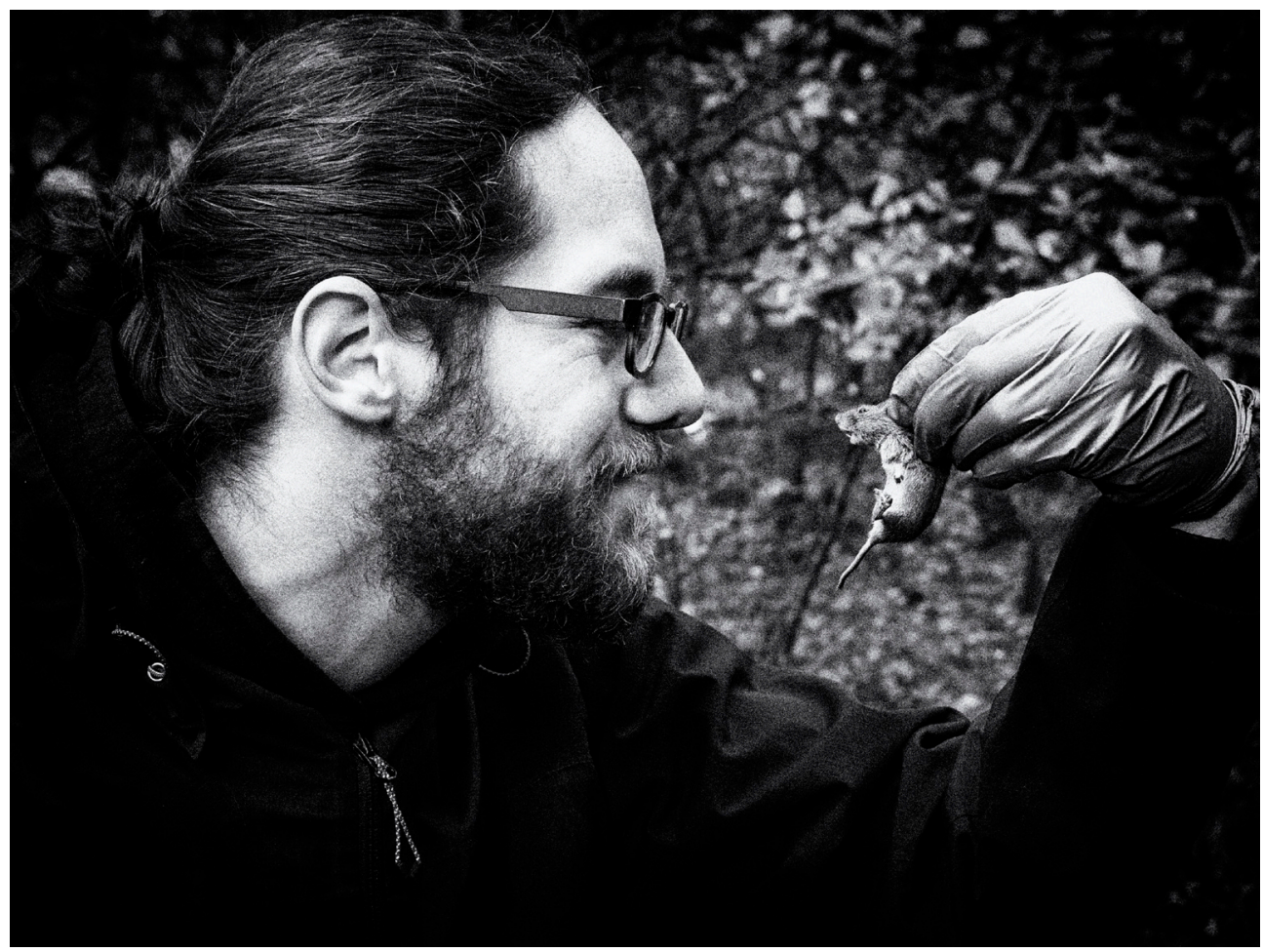




\section{BIOGRAPHY}

Tim Ragnvald Hofmeester was born on 13 June 1987 in Amsterdam, the Netherlands. Already at an early age, he became interested in nature, especially birds, which resulted in a membership of the Dutch Youth Organisation for Nature Studies (NJN) at the age of 12. During his years with the NJN he developed a special affinity with mammal research. After graduating with a gymnasium diploma at the Goois Lyceum in Bussum, it was a logical step to study Biology, first at the University of Amsterdam, and later at Wageningen University, where he obtained his MSc in Ecology (cum laude) in 2011. During his MSc he specialized in animal ecology with MSc theses on the spatial behaviour of Lions, Zebra and Wildebeest in Welgevonden, South Africa (supervised by dr Fred de Boer at the Resource Ecology Group (REG) of Wageningen University) and two theses on his favourite animal, the European pine marten: one on variation in fur patterns of Pine martens (supervised by dr Frank van Langevelde at REG) and one on the population genetics of the Dutch Pine marten population using noninvasive methods (supervised by dr Fons Debets at the Laboratory of Genetics of Wageningen University and drs Hugh Jansman at the Animal Ecology Group of Alterra).

In his free time Tim became a member of the Dutch Pine Marten Working Group (since 2006) and a member and board member of the Dutch Small Mustelid Working Group (since 2008), showing his love for small carnivores. After obtaining his MSc, Tim worked as a freelancer with his own company doing field work and data analyses on mammals for Alterra and the Dutch Mammal Society. But his true mission has always been to work at a university to combine his passions for research and education. Therefore, he was thrilled when he was asked by prof. dr. Herbert Prins to start his PhD project on the ecology of ticks 


\section{6 | Biography}

and tick-borne diseases at Wageningen University, where he started in March 2012.

During his work as a PhD candidate within the 'Shooting the messenger' project under the supervision of prof. dr Herbert Prins and dr Sip van Wieren at the Resource Ecology Group of Wageningen University, Tim was an organising member of the Wageningen Evolution and Ecology Seminars (WEES), and the second Tick Tactics symposium, giving PhD candidates from the Netherlands, Belgium and Germany the opportunity to present their work on ticks and tickborne diseases for an enthusiastic group of scientists and public health officials. The 'Shooting the messenger' project was a collaboration between the Laboratory of Entomology and the Resource Ecology Group of Wageningen University and the Centre for Infectious Disease Control Netherlands at the National Institute for Public Health and Environment (RIVM) and was funded by the Netherlands Ministry of Health, Welfare and Sport. The most important results from Tim's work in the 'Shooting the messenger' project are presented in this thesis.

While finishing his PhD thesis, Tim obtained a personal grant from the 'De Vos' foundation to perform an extra study within the 'Shooting the messenger' project on the interaction between ticks, tick-borne pathogens and innate immunity of wild rodents, which he performed in collaboration with dr Kevin Matson at the Resource Ecology Group of Wageningen University, and dr Miriam Maas and dr Hein Sprong at the Centre for Infectious Disease Control Netherlands at the RIVM. In the coming years, Tim hopes to combine his interest in ecto-parasites, zoonotic diseases, small mammals and carnivores to study the behavioural changes of disease reservoirs in response to the presence of predators.

In his free time Tim likes to be creative as a photographer and a musician.

\section{List of publications}

Peer review:

T.R. Hofmeester (in press) The role of host diversity in Borrelia burgdorferi s.I. dynamics. In: Ecology and prevention of Lyme borreliosis (eds. M.A.H. Braks, S.E. van Wieren, W Takken \& H. Sprong), Ecology and Control of vector-borne diseases Volume 4, Wageningen, the Netherlands, Wageningen Academic Publishers. 
T.R. Hofmeester, M.J. Rowcliffe \& P.A. Jansen (in press) A simple method to determine the effective detection distance of camera traps. Remote sensing in Ecology and Conservation. doi: 10.1002/rse2.25

T.R. Hofmeester, E.C. Coipan, S.E. van Wieren, H.H.T. Prins, W. Takken \& H. Sprong (2016) Few vertebrate species dominate the Borrelia burgdorferi s.l. life cycle. Environmental Research Letters, 11, 043001. doi: 10.1088/17489326/11/4/043001

T.R. Hofmeester, P. van der Lei, A. Docter van Leeuwen, H. Sprong \& S.E. van Wieren (2016) New foci of Haemaphysalis punctata and Dermacentor reticulatus in the Netherlands. Ticks and Tick-borne Diseases, 7, p367-370. doi:10.1016/j. ttbdis.2015.12.009

G.A. de Groot, T. R. Hofmeester, M. La Haye, H.A.H. Jansman, M. Perez-Haro \& H.P. Koelewijn (2016) Hidden dispersal in an urban world: genetic analysis reveals occasional long-distance dispersal and limited spatial substructure among Dutch pine martens. Conservation Genetics, 17, p111-123. doi: 10.1007/ s10592-015-0765-6

J.J.A. Dekker \& T.R. Hofmeester (2014) The status of the American mink (Neovison vison) in the Netherlands. Lutra, 57, p5-15.

A. Swart, A. Ibañez-Justicia, J. Buijs, S.E. van Wieren, T.R. Hofmeester, H. Sprong \& K. Takumi (2014) Predicting tick presence by environmental risk mapping. Frontiers in Public Health, 2. doi: 10.3389/fpubh.2014.00238

In advanced stage or submitted:

T.R. Hofmeester, P.A. Jansen, H.J. Wijnen, E.C. Coipan, M. Fonville, H.H.T. Prins, H. Sprong \& S.E. van Wieren (in revision) Cascading effects of changes in vertebrate assemblages on rodent-transmitted tick-borne disease risk.

E.C. Coipan, G. van Duijvendijk, A.Y. Krawczyk, T.R. Hofmeester, A. Swart, K. Takumi \& H. Sprong (in revision) Contribution of rodent species to maintenance of tick-borne pathogens in enzootic cycles.

T.R. Hofmeester \& P.A. Jansen (in preparation) Using camera traps to measure the availability of vertebrates hosts to ticks. 


\section{8 | Biography}

T.R. Hofmeester, H. Sprong, P.A. Jansen, H.H.T. Prins \& S.E. van Wieren (in preparation) Deer presence rather than abundance determines the population density of the sheep tick (Ixodes ricinus).

T.R. Hofmeester, P.A. Jansen, H.H.T. Prins, H. Sprong \& S.E. van Wieren (in preparation) Determinants of tick (Ixodes ricinus) burden on rodent populations in Dutch forests.

T.R. Hofmeester, E.C. Coipan, M. Fonville, P.A. Jansen, H.H.T. Prins, H. Sprong \& S.E. van Wieren (in preparation) No support for a dilution effect of host species richness on tick-borne disease risk in Europe.

Other publications:

T. Hofmeester \& J.J.A. Dekker (2016) De Bunzing. In: Atlas van de Nederlandse Zoogdieren (eds. S. Broekhuizen, K. Spoelstra, J.B.M. Thissen, K.J. Canters \& J.C. Buys), Zeist, the Netherlands, KNNV uitgeverij.

E. van Maanen, J. Mos, T. Hofmeester, F. Biljam, C. van den Tempel, F. van der Knaap \& J. Prescher (2015) Waar zijn de kleine marters? Zoogdier.

T. Hofmeester \& S. van Wieren (2014) Teken op Nederlandse zoogdieren. Zoogdier.

T. Hofmeester (2014) Waar zitten de teken. De Levende Natuur.

A. de Groot, H. Jansman \& T. Hofmeester (2014) Verwantschap en herkomst van de Limburgse populaties van het wild zwijn. Natuurhistorisch Maandblad.

H.A.H. Jansman, T. Hofmeester, G.A. de Groot, I. Laros, J. Bovenschen, M. Speelman, J. van der Hout, J. Caesar, P. Breyne \& H.P. Koelewijn (2013) Genetica van wilde zwijnen in Limburg en Noord-Brabant; Verspreiding, herkomst en verwantschap. Wageningen, Alterra, Alterra-rapport 2404. 



\section{PE\&RC Training and Education Statement}

With the training and education activities listed below the $\mathrm{PhD}$ candidate has complied with the requirements set by the C.T. de Wit Graduate School for Production Ecology and Resource Conservation (PE\&RC) which comprises of a minimum total of 32 ECTS (= 22 weeks of activities)

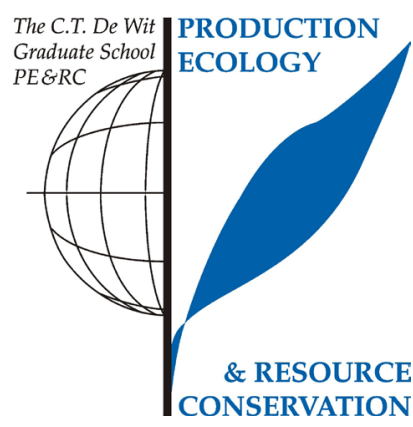

\section{Review of literature (4.5 ECTS)}

- Tick-host-pathogen interactions (2012)

\section{Writing of project proposal (4.5 ECTS)}

- Tick-host-interactions and the community ecology of a highly diversified tickborne pathogen (2012)

\section{Post-graduate courses (5.7 ECTS)}

- Introduction to R for statistical analysis; PE\&RC (2012)

- Survival analysis; PE\&RC (2013)

- Host-microbe interactions; EPS (2014)

- One health course; Institute of Virology, University of Bonn, Germany (2014)

- Bayesian statistics; PE\&RC (2014)

\section{Invited review of (unpublished) journal manuscript (2 ECTS)}

- Experimental and Applied Acarology: the natural infection of birds and ticks feeding on birds with Rickettsia sp. and Coxiella burnetii in Slovakia (2015)

- European Journal of Wildlife Research: camera traps and activity signs to estimate wild boar abundance and population trends (2016)

\section{Deficiency, refresh, brush-up courses (2.5 ECTS)}

- Basic statistics (2012)

- Epidemics - the dynamics of infectious diseases; Coursera (2014) 


\section{2 | PE\&RC Training and Education Statement}

Competence strengthening / skills courses (2.1 ECTS)

- Competence assessment; WGS (2012)

- Project \& time management; WGS (2013)

- Orientation on teaching for PhD candidates; ESD (2015)

- Writing grant proposals; WGS (2016)

PE\&RC Annual meetings, seminars and the PE\&RC weekend (1.8 ECTS)

- PE\&RC Annual meeting $(2012,2014,2015)$

- PE\&RC Last year weekend (2015)

Discussion groups / local seminars / other scientific meetings (5.5 ECTS)

- Ecological theory \& application (2012-2014)

- Wageningen evolution and ecology seminars (2012-2016)

- R User group (2013)

- Animal movement ecology (2013-2014)

International symposia, workshops and conferences (7.3 ECTS)

- XII International Jena symposium on tick-borne diseases (2013)

- Tick tactics 2; organizer (2014)

- Genes, ecosystems and risk of infection (2015)

- European congress of mammalogy (2015)

Lecturing / supervision of practicals / tutorials (3 ECTS)

- Wildlife resource management (2012-2015) 


\section{PE\&RC Training and Education Statement | 223}

\section{Supervision of MSc students}

- Ticks on mice and voles: the role of vegetation structure in parasite-host interactions (2013)

- The effect of mammal biodiversity on rodent parasite burden (2014)

- Where, when and why do mammals cross the road; analysis of mammal road kills in the Netherlands (2014)

- Deer and rabbit density as determinants of tick density on a local scale (2015)

- Habitat characteristics and increasing distribution ranges for Haemaphysalis punctata and Dermacentor reticulatus in the Netherlands (2015)

- Ixodid ticks of pine marten (Martes martes) and stone marten (M. foina) (2015)

- The edge effect of deer exclosures on the abundance of Ixodes ricinus and tick infestation of rodents (2016)

- Effects of generalist predator activity on Apodemus sylvaticus and Myodes glareolus densities in a woodland area in the Netherlands (2016) 
The research described in this thesis was financially supported by the Netherlands Ministry of Health, Welfare and Sport.

Cover design, layout, photos and figures:

Tim R. Hofmeester

Printed by:

Digiforce B.V. | | Uitgeverij Boxpress 